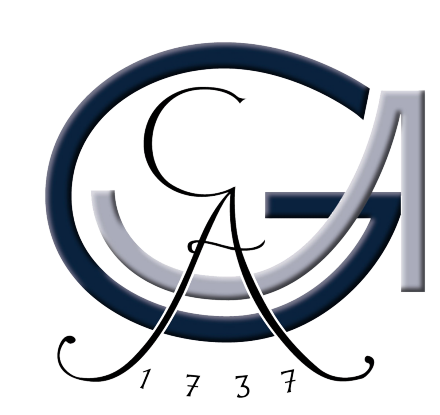

\title{
Studying Molecular Interactions under Flow with Fluorescence Fluctuation Spectroscopy
}

\author{
Dissertation \\ for the award of the degree \\ "doctor rerum naturalium" \\ of the Georg-August-Universität Göttingen
}

within the doctoral program

Göttingen Graduate School of Neuroscience, Biophysics and Molecular Bioscience (GGNB)

of the Georg-August-University School of Science (GAUSS)

\author{
submitted by \\ Eleonora Perego \\ from Milano (Italy)
}

Göttingen, 2019 


\section{Members of the Thesis Committee:}

Prof. Dr. Sarah Köster

Institute for X-ray Physics

Georg-August-Universität, Göttingen

Prof. Dr. Silvio Rizzoli

Department of Neuro- and Sensory Physiology

University Medical Center, Göttingen

\section{Prof. Dr. Christian Griesinger}

NMR-based Structural Biology

Max Plank Institute for Biophysical Chemistry, Göttingen 


\section{Members of the Examination Board:}

Referee: Prof. Dr. Sarah Köster

Institute for X-ray Physics

Georg-August-Universität, Göttingen

$2^{\text {nd }}$ referee: Prof. Dr. Silvio Rizzoli

Department of Neuro- and Sensory Physiology

University Medical Center, Göttingen

\section{Further members of the Examination Board:}

\section{Prof. Dr. Christian Griesinger}

Department of NMR-based Structural Biology

Max Plank Institute for Biophysical Chemistry, Göttingen

Dr. Sebastian Kruss

Institute for Physical Chemistry

Georg-August-Universität, Göttingen

Dr. Sarah Adio

Institute for Microbiology and Genetics

Georg-August-Universität, Göttingen

Dr. Helmut Grubmüller

Department of Theoretical and Computational Biophysics

Max Planck Institute for Biophysical Chemistry, Göttingen

Date of Oral Examination:

16 January 2020 


\section{Contents}

\begin{tabular}{lll}
\hline & Introduction & 1
\end{tabular}

\begin{tabular}{|lll}
2 & Theoretical background and state of the art & $\mathbf{3}$
\end{tabular}

$2.1 \quad$ Fluorescence fluctuation spectroscopy . . . . . . . . . . . . . . . . . 3

2.1 .1 The principles of fluorescence . . . . . . . . . . . . . . . . . . 4

2.1 .2 Fluorescence correlation spectroscopy . . . . . . . . . 7

$2.1 .3 \quad$ Photon counting histogram . . . . . . . . . . . . . . . . . . . . . . . . . . . . . 18

2.2 Microfluidics . . . . . . . . . . . . . . . . . . . . 18

$2.2 .1 \quad$ Flow at low Reynolds number . . . . . . . . . . . . . . . . 19

2.2 .2 Flow profile at low Reynolds number . . . . . . . . . . . . 19

2.2 .3 Mass transport in microfluidic devices . . . . . . . . . . 20

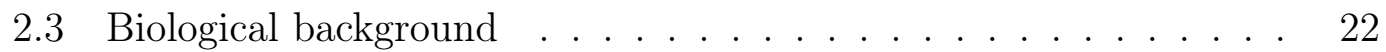

$2.3 .1 \quad$ Intermediate filaments . . . . . . . . . . . . . . . 22

2.3 .2 A brief introduction to synapse . . . . . . . . . . 26

3 Materials and methods 33

3.1 Protein preparation . . . . . . . . . . . . . . . . . . . . 33

3.1 .1 Vimentin protocols . . . . . . . . . . . . . . . . 33

3.2 Printing synaptic vesicles on glass surfaces . . . . . . . . . . . . . . . . . . . . 35

3.3 Microfluidic devices . . . . . . . . . . . . . . . . . . . . . 36

$3.3 .1 \quad$ Microfluidic device to study protein aggregation . . . . . . 37

3.3.2 Microfluidic device to study patterned SVs under a switchable flow . . . . . . . . . . . . . . . . . . 39

3.4 Setup for FFS and experimental conditions . . . . . . . . . . . . . 42

3.4 .1 Confocal microscopy . . . . . . . . . . . . . . . . . . 42

3.4 .2 Epi-fluorescence microscopy . . . . . . . . . . . . . . . . . . . . . . . . . . . 44

3.4 .3 Data acquisition and analysis . . . . . . . . . . . . . . . . . . . . . . . . . 44

3.5 Finite element methods simulations . . . . . . . . . . . . . . . . 46 
4 Results

$4.1 \quad$ Studying protein interactions and labeling stoichiometry under flow 49

$4.1 .1 \quad$ Microfluidic device optimization . . . . . . . . . . . 50

$4.1 .2 \quad$ PCH measurements of vimentin tetramers in bulk . . . . . 57

4.1 .3 Vimentin tetramers in flow . . . . . . . . . . . 64

4.1.4 Measurements of vimentin lateral assembly under flow . . 71

4.1 .5 Discussion . . . . . . . . . . . . . . . 80

4.2 Protein interactions in the synapse . . . . . . . . . . . . 85

$4.2 .1 \quad$ Measuring protein diffusion in living neurons . . . . . . 85

4.2 .2 Measuring in vitro protein-vesicle interactions . . . . . . . . . 88

$4.2 .3 \quad$ Synaptic vesicles under switchable flow . . . . . . . . . . . 98

\begin{tabular}{lll}
\hline 5 & Summary, conclusions and outlook & 107
\end{tabular}

\begin{tabular}{ll}
\hline A Appendix & 117
\end{tabular}

A.1 Microcontact printing . . . . . . . . . . . . . . . . . . . . 117

A.2 Detailed FCS derivation . . . . . . . . . . . . . . . . . . . . . . . 118

A.3 Detailed derivation of the photon count distribution . . . . . . . . 120

\begin{tabular}{lr}
\hline Bibliography & 129
\end{tabular} 


\section{Abbreviations and Symbols}

\section{Abbreviations}

$\alpha$-syn Alpha-synuclein

ACF Auto Correlation Function

AFM Atomic Force Microscope

BSA Bovine Serum Albumin

CCF Cross Correlation Function

DLS Dynamic Light Scattering

EM Electron Microscopy

FCA Fluorescence Comulants Analysis

FCS Fluorescence Correlation Spectroscopy

FEM Finite Element Method

FFS Fluorescence Fluctuation Spectroscopy

FIDA Fluorescence Intensity Distribution Analysis

FRAP Fluorescence Recovery After Photobleaching

FRET Foerster Resonance Energy Transfer

ICCS Image Cross-Correlation Spectroscopy

LR Labeling Ratio

MIMIC MIcroMolding In Capillary

MW Molecular Weight

NA Numerical Aperture

OPE One Photon Excitation

PB Phosphate Buffer

PBS Phosphate Buffered Saline

PCH Photon Counting Histogram

PDMS PolyDiMethylSiloxane

PSF Point Spread Function

RICS Raster scan Image Correlation Spectroscopy

ROI Region Of Interest

ULF Unit Length Filament 
SAXS Small Angle X-rays Scattering

SLS Static Light Scattering

STED STimulated Emission Depletion

STEM Scanning Transmission Electron Microscopy

SV Synaptic Vesicle

TIRF Total Internal Reflection Fluorescence 


\section{List of Symbols}

\begin{tabular}{|c|c|}
\hline$\gamma$ & OPE factor \\
\hline$\gamma_{S}$ & shear rate \\
\hline$\Gamma$ & rate of emitted photons \\
\hline$\delta F$ & fluctuation of fluorescence intensity \\
\hline$\varepsilon$ & molar extinction coefficient \\
\hline$\eta$ & viscosity \\
\hline$\eta_{W}$ & energy detection efficiency \\
\hline$\eta_{I}$ & intensity detection efficiency \\
\hline$\Phi$ & quantum yield \\
\hline$\Theta$ & fraction of molecule in the dark state \\
\hline$\lambda$ & wavelength of light \\
\hline$\mu$ & mobility \\
\hline$\mu-\mathrm{CP}$ & micro-contact printing \\
\hline $\bar{\nu}$ & specific gravity \\
\hline$\rho$ & density \\
\hline$\sigma_{a b s}$ & absorption cross section \\
\hline$\tau$ & FCS lagtime \\
\hline$\tau_{D}$ & diffusion time \\
\hline$\tau_{\text {Dmix }}$ & diffusion mixing time \\
\hline$\tau_{D t}$ & detector dead-time \\
\hline$\tau_{\mathrm{F}}$ & flow time \\
\hline$\tau_{\text {fluc }}$ & fluorescence fluctuation time scale \\
\hline$\tau_{\text {fluo }}$ & fluorescence lifetime \\
\hline$\tau_{\text {phot }}$ & triplet component time \\
\hline$X_{\text {phot }}$ & triplet component - ACF \\
\hline$\tau_{S}$ & shear stress \\
\hline$\omega_{0}$ & PSF radial diameter \\
\hline$\omega_{c}$ & channel width \\
\hline$A$ & absorbance \\
\hline$B$ & molecular brightness per sampling tim \\
\hline
\end{tabular}


$b \quad$ molecular brightness

c concentration

$c_{d} \quad$ drag coefficient

$D \quad$ diffusion coefficient

$F \quad$ OPE correction factor

$G(\tau)_{D} \quad$ diffusion component - ACF

$I_{D} \quad$ light intensity at the detector plane

$k_{D} \quad$ transition rate to the dark state

$k_{b} \quad$ transition rate to the bright state

$k_{B} \quad$ Boltzmann constant

$N \quad$ average number of molecule per observation volume

$N_{A} \quad$ Avogadro number

$p \quad$ pressure

$P e \quad$ Péclet number

$p(W(t))$ energy distribution on the detector

$Q \quad$ flow rate

$R \quad$ hydrodynamic radius

Re Reynolds number

$S \quad$ sources or sink of mass in the diffusion-convection equation

$S(\vec{r}) \quad$ optical transfer function

$S_{0}\left(k_{n r}\right) \quad$ rate for non-radiative decay

$t \quad$ time

$T \quad$ sampling time or integration time

$v \quad$ velocity

$V \quad$ volume

$V_{\text {eff }} \quad$ effective observation volume

$W(\vec{r}) \quad$ spatial distribution of the light intensity

$z_{0} \quad$ PSF axial dimension 


\section{List of Figures}

$2.1 \quad$ Jablonski diagram. . . . . . . . . . . . . . . . . . 5

$2.2 \quad$ Illumination volume and observed volume with confocal detection. 8

2.3 Example of FCS curves for different parameters. . . . . . . . . . 9

2.4 ACF with and without convective flow. . . . . . . . . . . 11

$2.5 \quad$ FCS and PCH for two populations with different brightnesses and same diffusion coefficient. . . . . . . . . . . . . . . . . 13

2.6 Images of cytoskeletal filaments in 3T3 mouse fibroblast cell. . . . 23

2.7 Scheme of vimentin assembly in vitro. . . . . . . . . . . . . . . . 25

2.8 Basic illustration of a neuron. . . . . . . . . . . . . . . 26

2.9 Schematic representation of a chemical synapse. . . . . . . . . 27

2.10 3D model for an average synapse. . . . . . . . . . . . . . . 28

2.11 Two possible conformations of human $\alpha$-synuclein. . . . . . . 30

3.1 Vimentin filaments after $8 \mathrm{~h}$ of in vitro assembly. . . . . . . . . . 34

3.2 Patterning of a glass coverslip using the PRIMO system. . . . . . 36

3.3 Main photo-lithography steps to produce device B. . . . . . . . 38

$3.4 \quad$ Five-inlet microfluidic device to study protein aggregation. . . . . 40

$3.5 \quad$ Microfluidic device to study SVs under a switchable flow. . . . . . . 41

3.6 Device holder for microfluidic chips with patterned molecules on

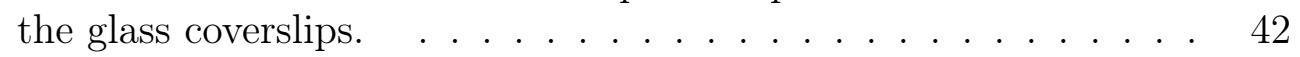

3.7 Schematic representation of the confocal microscopy setup. . . . . 43

3.8 First section of the channel geometry used in a FEM simulation. . 47

4.1 Vimentin assembling in flow inside a no-step microfluidic device. . $\quad 50$

4.2 Step microfluidic device employed to study assembly of vimentin. 51

4.3 Result of a FEM simulation for the step five-inlet microfluidic device at slow flow rates. . . . . . . . . . . . . . 52

4.4 Results of a FEM simulation for the step device B. . . . . . . . 53

4.5 Results of a FEM simulation for the step device at high flow rates. 54 
4.6 Comparison of vimentin concentration along $z$ in the microfluidic

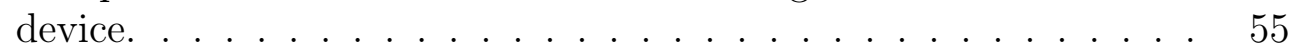

4.7 Vimentin concentration along the center of the microfluidic device. 56

4.8 PCH and FCS simulation results and fits. . . . . . . . . . . . . 58

4.9 Vimentin filaments after 7 hours of assembly at $37^{\circ} \mathrm{C}$. . . . . . . . 60

$4.10 \mathrm{PCH}$ and fits for simulated vimentin assemblies at different assembling stages for different LRs. . . . . . . . . . . . . . . . . . 61

$4.11 \mathrm{PCH}$ of vimentin tetramers in bulk at three different concentrations. 62

$4.12 \mathrm{PCH}$ and FCS curves of vimentin tetramers in bulk at different

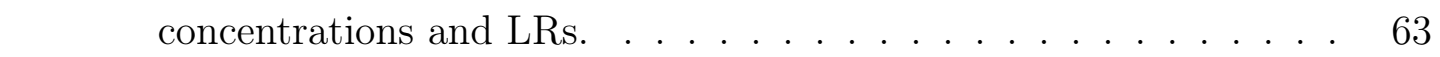

4.13 Fluorescence intensity trace over time for a sample of fluorospheres in a channel with and without velocity. . . . . . . . . . . 65

4.14 Plug velocity profile. . . . . . . . . . . . . . 66

$4.15 \mathrm{PCH}$ and FCS curves of vimentin tetramers in flow at different

velocities. ......................... 66

4.16 Brightness and number of fluorophores per vimentin sub-unit for vimentin tetramers in the five-inlet step microfluidic device. . . . 68

4.17 Number of molecules per observation volume for vimentin tetramers in the step device. . . . . . . . . . . . . . . . . . 69

4.18 NOA glue step inside the microfluidic device during an experiment with vimentin tetramers in flow. . . . . . . . . . . 70

4.19 Brightness and average number of molecules per observation volume for the second component of vimentin tetramers in flow. . . . 71

4.20 Brightness and number of labels per molecules over time for vimentin assembling in a microfluidic device $(\mathrm{LR}=28 \%) . \quad \ldots . .73$

4.21 Brightness and number of labels per molecules over time for vimentin assembling in a microfluidic device ( $\mathrm{LR}=12 \%) . \quad \ldots$. . . 74

4.22 Brightness over time for vimentin assembling in a microfluidic device $(\mathrm{LR}=40 \%) . \ldots \ldots \ldots \ldots \ldots \ldots \ldots \ldots$

4.23 Average number of fluorescent particles per observation volume during the assembly, for vimentin at $12 \%$ and $28 \%$. . . . . . . 76

4.24 Normalized average number of molecules per observation volume over time for two vimentin assembly experiments. . . . . . . . . 77

4.25 FCS curves and PCH of vimentin before and after the microfluidic device. . . . . . . . . . . . . . . 77

4.26 Brightness and average number of molecules per observation volume over time for the second component of the low labeling ratio

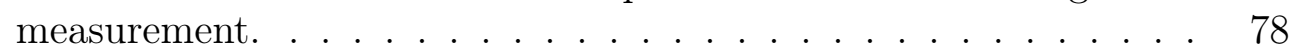

4.27 Example of exponential curve fit on the brightness over time for a vimentin assembling experiment and histogram of the vimentin lateral assembly time scales. . . . . . . . . . . . . . . . . 79

4.28 Relation between the time scales for the ULF formation and labeling ratio. . . . . . . . . . . . . . . . . . 82 
4.29 Typical epi-fluorescence image of hippocampal cultured neurons expressing mEGFP. . . . . . . . . . . . . . . . . 86

4.30 Normalized average FCS curves acquired on neurons expressing mEGFP-SNAP25, mEGFP, membrane-mEGFP or EGFP-synapsin.

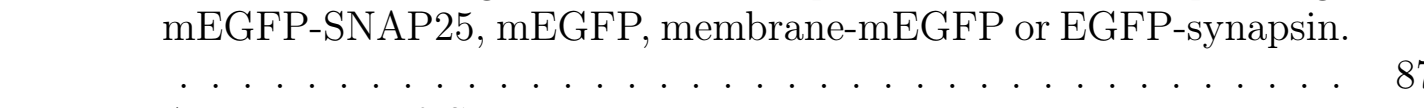

4.31 Attachment of SVs to glass coverslip and a typical neutravidin

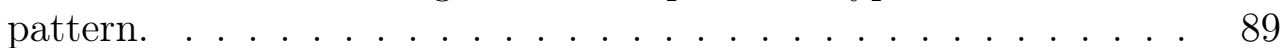

4.32 Epi-fluorescence images of patterns on glass coverslips. . . . . . . 90

4.33 Epi-fluorescent images of different antibodies on the patterns. . . 92

4.34 Normalized and averaged FCS curves of $\alpha$-synuclein-Alexa532, $\alpha$ ms-Atto532 and gt $\alpha$-rat-Atto532 measured on a SV pattern. . . . 93

4.35 Normalize FCS average curves of $\alpha$-synuclein-eGFP purified and $\alpha$-synuclein-eGFP from HEK cell lysate freely diffusing in bulk. . .94

4.36 Normalized FCS average curves of $\alpha$-synuclein-eGFP freely diffusing in bulk and diffusing on top a SV pattern. . . . . . . . . . 96

4.37 Epi-fluorescence images of SV and $\alpha$-synuclein patterns taken after FCS measurements. . . . . . . . . . . . . . . 97

4.38 Input velocity profile applied to the inlets in the microfluidic device employed to switch buffers on the SV patterns. . . . . . . . . . 100

4.39 Concentration of fluorescein in simulated microfluidic device at different time points. . . . . . . . . . . . . . . . . 101

4.40 Epi-fluorescence images of fluorescein flowing in a microfluidic device with switching flow. . . . . . . . . . . . . . . 102

4.41 Epi-fluorescence images of the SV patterns before and after 30 minutes in the microfluidic device with PBS flowing on the pattern at a velocity of $4 \mathrm{~mm} / \mathrm{s} . \quad \ldots \ldots \ldots \ldots 104 \ldots$

4.42 Epi-fluorescence images of the antibody patterns before and after 30 minutes in the microfluidic device with PBS flowing on the pattern at a velocity of $4 \mathrm{~mm} / \mathrm{s} . \ldots \ldots . . \ldots 105$

A.1 Schematic representation of $\mu$-CP. . . . . . . . . . . . . . . 118 


\section{List of Tables}

2.1 SHC (sequence homology class) classes of IFs, examples of the IFs in that class and their occurrence in mammalian cells (adapted from [80]). . . . . . . . . . . . . . . . . . . . . . . . 24

3.1 Spin coating velocities used to achieve the corresponding resist heights. . . . . . . . . . . . . . . . . . . 39

4.1 Fitting results from the analysis of the simulation shown in Figure [4.81. . . . . . . . . . . . . . . . . . . . . . . . . . . . . . . . 59

4.2 Summary of the brightness $(B)$ and the average number of labels $(N)$ per vimentin molecule at different assembly stages for three

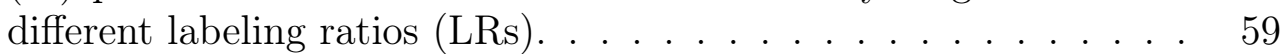

4.3 Brightness $(B)$ and number of molecules per observation volume $(N)$ of simulated vimentin tetramers and ULF retrieved with a two components model fit. . . . . . . . . . . . . . 60

$4.4 \quad$ Fit parameters of vimentin tetramer PCHs in bulk. . . . . . . . . 63

\begin{tabular}{|lll|l|}
\hline 4.5 & Summary of the fitting parameters of curves shown in Figure & 4.12 & 64
\end{tabular}

4.6 Parameters retrieved from the analysis of PCHs and FCS curves

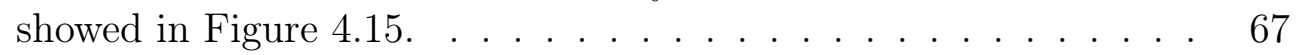




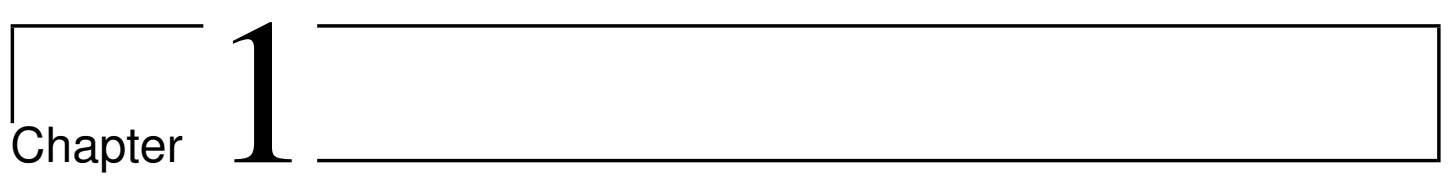

\section{Introduction}

Assembly and interactions of biomolecules are fundamental processes in living organisms to maintain the physiological cellular behavior. The majority of the time protein assembly is useful and vital for the organism, like the formation of the cytoskeletal filaments in cells or the assembly of the mitotic spindle during division. However, sometimes atypical aggregates are produced. These protein aggregates are usually generated from a fault during the normal assembly pathway [1. Typically, the human body has mechanisms to detect these modifications and discard them, but sometimes the aggregates can be unseen and subsequently lead to pathological situations [2]. The diseases caused by protein aggregation are not always life threatening, as in the case of cataracts, which is the leading cause of blindness worldwide [3. In other cases, protein aggregation is responsible for more severe diseases. A typical example is the aggregation of proteins from the synuclein family, which are considered one of the main factors in the outbreak of some neurodegenerative diseases [4. This family of diseases, which are called synucleinopathies, includes Parkinson's disease, dementia with Lewy bodies, a variant of Alzheimer's disease and others. Aggregates of $\alpha$-synuclein are usually found in unsoluble and toxic inclusions in the brain called Lewy bodies [5].

It is fundamental to study both the ordered protein assembly and the disordered protein aggregation with spatial resolution on the order of some nm to gain a complete knowledge of the resulting assemblies at a single molecule level. Furthermore, good time resolution of the reaction kinetics, on the order of ms, is also very important. In particular for the pathological aggregation it is fundamental to find out the exact moment when the assembly changes from ordered to disordered, in order to try to prevent the disease caused by the faulty aggregation.

In this thesis, fluorescence fluctuation spectroscopy (FFS) is combined with microfluidics to study protein interactions and assembly with high temporal resolution. FFS is a family of techniques which are all based on studying the fluctuations of the fluorescence signal detected within a confined excitation volume [6]. The fluctuations can be, for example, correlated in time as in fluorescence correla- 
tion spectroscopy (FCS) to obtain information about protein mobility [7]. Alternatively, the frequency of the fluctuations can be evaluated, as in photon counting histogram $(\mathrm{PCH})$ or in fluctuation intensity distribution analysis (FIDA) [8, 9] to measure the brightness of the fluorescence molecules. FFS is often applied to quantify aggregation [10, 11, 12], however, all the techniques from this family lack high temporal resolution, since long acquisition times are usually needed for a good signal-to-noise ratio. Thus, microfluidic methods are integrated into our measurements to access different time points in protein reactions. Microfluidics is largely applied to study biological samples since it offers multiple advantages, such as high throughput, low costs, short reaction times and a reliable control on the small volume employed [13]. Here, continuous flow microfluidics is employed to track the dynamics of protein interaction and assembly over time [14, 15, 16].

In Chapter 2, an overview of FFS and microfluidics is provided. In particular, the general aspects of fluorescence are introduced, as well as FCS and PCH. Afterwards, the biological systems studied in this thesis are introduced. First, intermediate filaments (IFs) are described, focusing on the hierarchical assembly of vimentin. Second, a brief introduction of synapses and synaptic vesicles is given. Third, in the last section of Chapter 2, $\alpha$-synuclein is presented, focusing in particular on the conformational changes of this protein. Chapter 3 contains the description of the protein preparation, the manufacturing of the microfluidic devices, the experimental procedures and the data analysis. The results of the experiments are presented in Chapter 4. In the first part of the chapter, experiments on vimentin assembly in flow are presented. The early time points of vimentin assembly are captured and the results are discussed in the context of current literature. In the second part of Chapter 4, the experiments on the protein interactions and mobility in synapses are described. Interactions between synaptic vesicles and $\alpha$-synuclein are measured on patterned glass substrates and a microfluidic chamber is build to access the temporal information of the reactions. Finally, in Chapter 5, the results of the experiments are summarized and the conclusions of this thesis are formulated and, as an outlook, possible new experiments and open questions are discussed. 


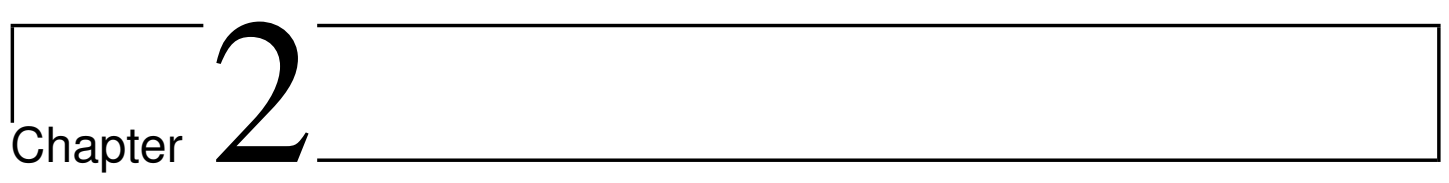

\section{Theoretical background and state of the} art

In this chapter, there will be a theoretical introduction to the techniques employed in this thesis and the biological samples studied. Moreover, the experiments will be contextualized within the current state of the art.

\subsection{Fluorescence fluctuation spectroscopy}

The sentence "one man's noise is another man's signal" [17] summarizes the general idea of fluorescence fluctuation spectroscopy (FFS). In fact, the techniques in the family of FFS instead of focusing on the average fluorescence intensity, are based on the fluctuations around the average signal. The historical starting point of this group of techniques can be pointed back to the first observations made by Robert Brown on the random movements of pollen grains suspended in water [18. The phenomenon later attracted the attention of Einstein and Smoluchwski who developed a theory describing these movements. In the 1950s and in the 1960s these fluctuation theories have been applied to light scattering, developing dynamic light scattering (DLS), which is used to measure diffusion coefficients of suspended samples [19]. Soon later, in the early 1970s, they were also applied to fluorescence by Magde, Elson and Webb with a series of three seminar papers [7, 21, 22] that describe theory and applications of fluorescence correlation spectroscopy (FCS) in the absence of external perturbation. The widespread application of FCS arrived only in the 1990s with the development of confocal [23] and two-photon [24 microscopy which reduce the observation volume, improving the signal-to-noise ratio and the sensitivity to single molecule fluctuations.

Since then FCS is applied to various types of samples and used to answer many questions both in vivo and in vitro. It can be applied to measure rotational and translational diffusion [25], molecular interactions [26, flow rates [27], kinetic 
processes [28] and aggregation formations [29]. FCS is now not only used with confocal or two-photon microscopy, but it can be applied to all microscopes, where the volume of excitation is well defined, as in total internal reflection (TIRF) [30] or stimulated-emission depletion microscopy (STED) [31]. While FCS focuses on the temporal correlation of the fluctuations, there are other techniques that exploit statistical tools to study directly the intensity of the fluctuations. The first approaches were made in the 1980s trying to analyze the moments of the photon counts [32]. Almost at the same time, two groups developed two very similar methods to analyze the distribution of the photon counts: photon counting histogram (PCH) [8] and fluorescence-intensity distribution analysis (FIDA) 9]. Another similar approach is called fluorescence cumulant analysis (FCA) [33]. While in $\mathrm{PCH}$ and FIDA, the probability distribution of the photon counts is calculated and then fitted, in FCA the photon counts are analyzed using the cumulant generating functions. Cumulants are similar to the moments describing probability functions and they are in particular used to characterize distributions of random, independent processes. With PCH, FIDA and FCA the number and the brightness, which is defined as the photon counts emitted per second per molecule, of the molecules can be determined without any information about the dynamics involved. A more straightforward approach to fluorescence fluctuations data is called Number and Brightness ( $\mathrm{N} \& \mathrm{~B}$ ) analysis [34, where just the first and the second moment (i.e. the mean and the variance) of the intensity fluctuations are needed to create a map of brightnesses and numbers at every pixel of an image. It can be applied directly to confocal images. If these techniques are used in combination with microfluidics, temporal information can be accessed. In the next sections a general introduction to fluorescence and two techniques based on fluorescence fluctuation, FCS and PCH, will be discussed in more detail.

\subsubsection{The principles of fluorescence}

Fluorescence is a process, where an excited electron returns to the ground state loosing energy in the form of photon. A molecule can be excited by a photon that carries an energy $h \nu$ equal to the difference between the molecular energy levels. This process is called absorption. It is a very fast process, the time scales are in the order of $10^{-15} \mathrm{~s}$. Since it is so fast, the molecules do not have time to undergo any structural changes (Franck-Condon principle). Absorption can be described by the Beer-Lambert law [6]:

$$
A=\log \left(\frac{I_{0}}{I}\right) \approx c \lambda \varepsilon l,
$$

where $A$ is a dimensionless quantity called absorbance, $I_{0}$ is the intensity of the incident light, $I$ is the intensity of the transmitted light after a distance $l, c$ is the molar concentration of the absorbing molecule, $\lambda$ is the wavelength of the absorbed light and $\varepsilon$ is the molar extinction coefficient. The last parameter is proportional to the one-photon absorption cross section of the specific molecule. This 


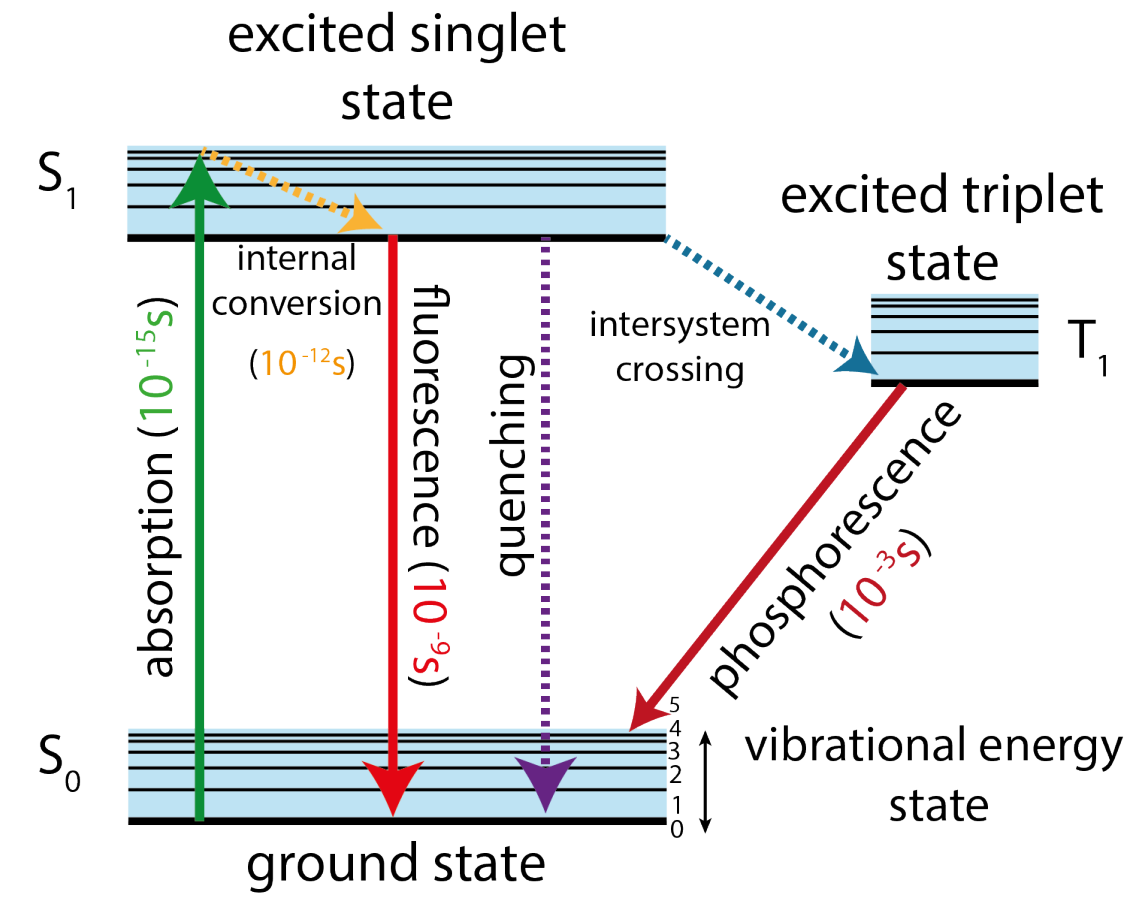

Figure 2.1: Jablonski diagram. It shows graphically the possible decaying paths for an excited molecule. The dotted arrows correspond to non-radiative decays. The molecule is excited by absorption of a photon that carries an energy equal to the energy difference between $S_{0}$ and $S_{1}$ (green arrow). From $S_{1}$, the molecule quickly relaxes in a non-radiative manner to the lower vibrational level of $S_{1}$ by internal conversion (orange dotted line). The excited molecule can relax to $S_{0}$ by fluorescence (red arrow), by non-radiative relaxation or by quenching (purple dotted arrow). Or, the molecule can decay from $\mathrm{S}_{1}$ to the triplet excited state $\left(\mathrm{T}_{1}\right)$ by intersystem crossing, and emits from there (phosphorescence, dark red arrow).

equation is actually used to calculate the concentration of an unknown sample measuring the absorbance. There are three main contributions to the absorption spectrum:

- Electronic: corresponds to the electronic gap transition. The light absorbed in the electronic gap is in the UV-visible light spectrum (between $200 \mathrm{~nm}$ to $900 \mathrm{~nm})$.

- Vibrational: corresponds to the transition between vibrational levels in the same electronic state; these transitions are caused by IR radiation.

- Rotational: corresponds to transition between rotational states within the same vibrational state. In this case the energies correspond to microwaves.

Both the excitation and the de-excitation of a molecule can be graphically described by the Jablonski diagram [41], shown in Figure 2.1. At room temperature, thermal energy is not sufficient to significantly populate the excited vibrational 
states in the ground state $\mathrm{S}_{0}$, thus absorption typically occurs from the lowest vibrational energy level. The energy gap between $S_{0}$ and the excited states $S_{1}$ is usually too big for thermal energy only, that is why also light is needed to induce absorption. The dotted lines in Figure 2.1 represent the non-radiative decaying pathways. Typically, the molecule is excited to an higher vibrational level in the $\mathrm{S}_{1}$ state (green arrows in Figure 2.1) and from there it rapidly relaxes to the lower vibrational level in the excited state. This process is called internal conversion (orange dotted in Figure 2.1) and generally occurs in $10^{-12} \mathrm{~s}$ or less. Fluorescence lifetimes are shorter than the internal conversion (around $10^{-9} \mathrm{~s}$ ), thus the return to the ground state via fluorescence (red arrows in Figure 2.1) typically occurs from the lower vibrational state in $S_{1}$. The emission spectrum is usually a mirror image of the absorption spectrum. Molecules in the $S_{1}$ state can also decay to the first triplet state $\mathrm{T}_{1}$ (inter-system crossing, dotted cyan arrow) and emit from there. The radial emission that occurs from this state is called phosphorescence, it is slower than fluorescence emission (occurs in times on the order of $10^{-3} \mathrm{~s}$ ) and it is shifted to longer wavelengths. The molecule can also lose energy from the $\mathrm{S}_{1}$ state via non-radiative emission, it can either be forced to relax to $\mathrm{S}_{0}$ (quenching, purple dotted arrow) or it can decay spontaneously to the ground state. Because of internal conversion, fluorescence typically occurs at lower energies than absorption, thus the emitted light has a longer wavelength than the absorbed light. This phenomenon is called Stokes shift and was first observed by Sir G. G. Stokes in 1852 in Cambridge [42]. The Stokes shift was fundamental during the development of fluorescence microscopy because it ensures the distinction between excited and emitted light 43 .

To characterize a fluorophore the most important parameters are the fluorescence lifetime and the quantum yield. The quantum yield $(\Phi)$ is the number of emitted photons relative to the number of absorbed photons. It is defined as the ratio between the rate of emitted photons $(\Gamma)$ and the total rate of absorption, i.e. the rate of emitted photons plus the rate of non-radiative decay $\left(S_{0}\left(k_{n r}\right)\right)$ :

$$
\Phi=\frac{\Gamma}{\Gamma+S_{0}\left(k_{n r}\right)} .
$$

Note that the quantum yield is always lower than 1 because of the non-radiative decay during the internal conversion. The lifetime determines the time available for the fluorophore to interact or diffuse in its environment before emitting. The lifetime of the excited state is defined by the average time the molecule spends in the excited state prior returning to the ground state. It can be expressed using again the emission rate and the rate for non-radiative decay:

$$
\tau_{\text {fluo }}=\frac{1}{\Gamma+S_{0}\left(k_{n r}\right)} .
$$

However, since fluorescence emission is a random process, and only few molecules emit their photons at precisely $t=\tau_{\text {fluo }}$, the lifetime is an average value of the time spent in the excited state. 


\subsubsection{Fluorescence correlation spectroscopy}

Fluorescence Correlation Spectroscopy (FCS) is a versatile technique based on the analysis of fluorescence fluctuations over time [6]. The fluctuations are the result of molecular dynamics in the excitation volume. They are usually mostly caused by Brownian motion of molecules moving across the observation volume shaped on the laser Point Spread Function (PSF). When a fluorophore passes trough the excitation volume, a burst of photons is emitted depending on how fast the molecules diffuse; slowly diffusing fluorophores will have longer pulses than fast fluorophores. By correlating the time dependent fluctuations it is possible to measure the diffusion coefficient of the molecule. For bulk experiments photo-bleaching is usually not an issue since molecules are quickly replaced by diffusion. It could be an issue, however, for fixed samples or cell measurements [36]. Intensity fluctuations could also be caused by other dynamics happening in the sample as molecular interactions, rotational diffusion or photo-physical effects [7. To properly measure the local changes in concentration, that usually are the cause of the intensity fluctuations, it is important to have a low concentration of emitters and a small confined excitation volume. Otherwise the fluctuations caused by a single fluorophore are not distinguishable anymore from the average intensity. The detailed mathematical derivation can be found in the Appendix A.2. The normalized auto-correlation function (ACF) can be defined as follows:

$$
G(\tau)=\frac{\langle\delta F(t) \cdot \delta F(t+\tau)\rangle}{\langle F(t)\rangle^{2}},
$$

where $\delta F(t)$ represents the fluctuation of fluorescence intensity in a certain time $t$ measured on the detector, and $\tau$ is the time delay relative to an earlier time point in the measurement. The fluctuations of the measured fluorescence intensity can be written as:

$$
\delta F(t)=\int_{V} W(\vec{r}) \delta(b C(\vec{r}, t)) d V
$$

Here, $W(\vec{r})$ is the spatial distribution of the emitted light intensity, or PSF, $V$ is the volume, $C$ is the concentration of the diffusing fluorophores and $b$ is the brightness of the molecule, defined as the product between the quantum efficiency of photon detection, the cross section of absorption and the quantum yield of emission $(\Phi)$. In our case, the brightness is considered constant and only concentration fluctuations are considered. Then, equation (2.4) can be written as:

$$
G(\tau)=\frac{\iint_{V V^{\prime}} W(\vec{r}) W\left(\overrightarrow{r^{\prime}}\right)\left\langle\delta C(\vec{r}, 0) \delta C\left(r^{\prime}, \tau\right)\right\rangle d V d V^{\prime}}{\left(\langle C\rangle \int_{V} W(\vec{r}) d v\right)^{2}},
$$

If the fluctuations of the concentration over time are only caused by 3D Brownian motion, then they can be written as: 


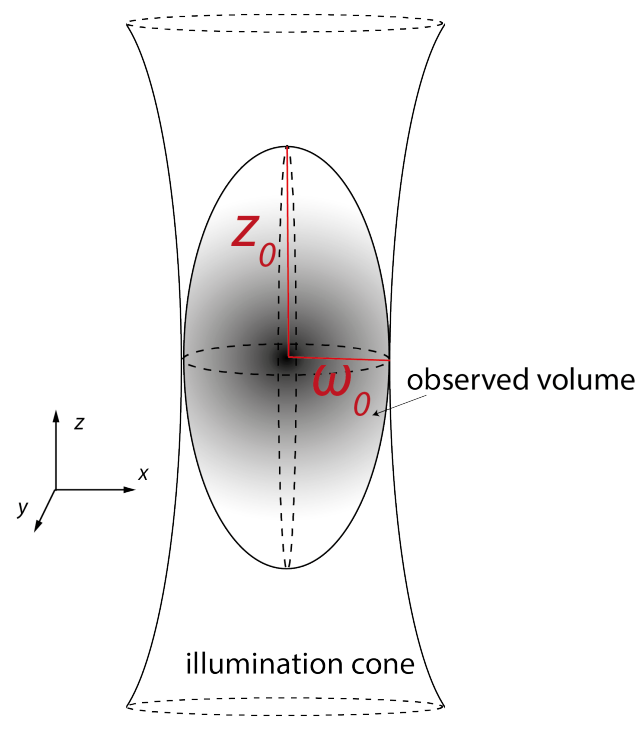

Figure 2.2: Illumination volume for one photon excitation and observed volume with confocal detection. $w_{0}$ and $z_{0}$ represent the beam profile parameters, defined as the position where the intensity is decreased by a factor $1 / e^{2}$.

$$
\left\langle\delta C ( \vec { r } , 0 ) \delta C \left({\left.r^{\prime}, \tau\right)}^{\overrightarrow{2}}=\langle C\rangle \frac{1}{(4 \pi \tau D)^{3 / 2}} e^{\left(\vec{r}-\vec{r}^{\prime}\right)^{2} / 4 D \tau},\right.\right.
$$

where $D$ is the diffusion coefficient. Then, it is possible to re-write equation (2.6) as:

$$
G(\tau)=\frac{1}{\langle C\rangle(4 \pi \tau D)^{3 / 2}} \frac{\iint_{V V^{\prime}} W(\vec{r}) W\left(\overrightarrow{r^{\prime}}\right) e^{\left(\vec{r}-\vec{r}^{\prime}\right)^{2} / 4 D \tau} d V d V^{\prime}}{\left(\int_{V} W(\vec{r}) d v\right)^{2}} .
$$

In our case $W(\vec{r})$ is described by a 3D Gaussian (confocal case):

$$
W(\vec{r})=I_{0} e^{-2\left(x^{2}+y^{2}\right) / w_{0}^{2}} e^{-2 z^{2} / z_{0}^{2}},
$$

where $\vec{r}=(x, y, z)$, and $w_{0}$ and $z_{0}$ are the beam profile parameters, as shown in Figure 2.2. Evaluating equation 2.8 with equation 2.9, it is finally possible to write the $\mathrm{ACF}$ for molecules diffusing in the volume defined by the PSF:

$$
G_{D}(\tau)=\frac{\gamma}{N} \frac{1}{1+\frac{\tau}{\tau_{D}}} \frac{1}{\sqrt{1+\frac{\tau}{\tau_{D}}\left(\frac{w_{0}}{z_{0}}\right)^{2}}}
$$

For the one photon excitation (OPE) case $\gamma=0.35$ ( $\gamma$ is a constant related to the PSF calculation) and $\tau_{D}=\frac{w_{0}^{2}}{4 D}$. 
a.

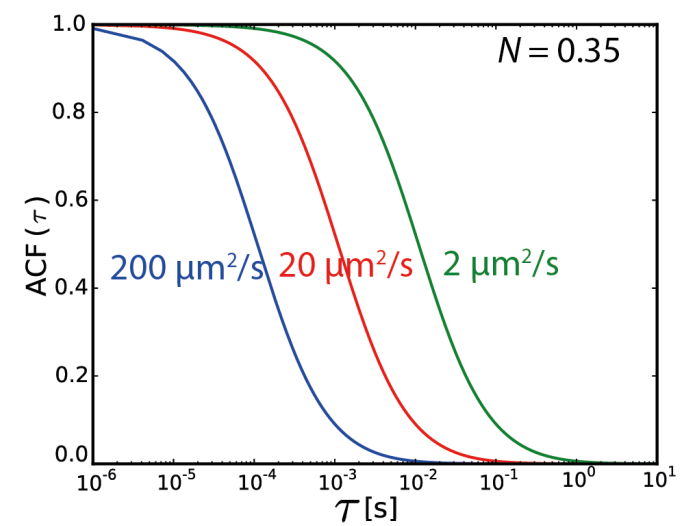

C.

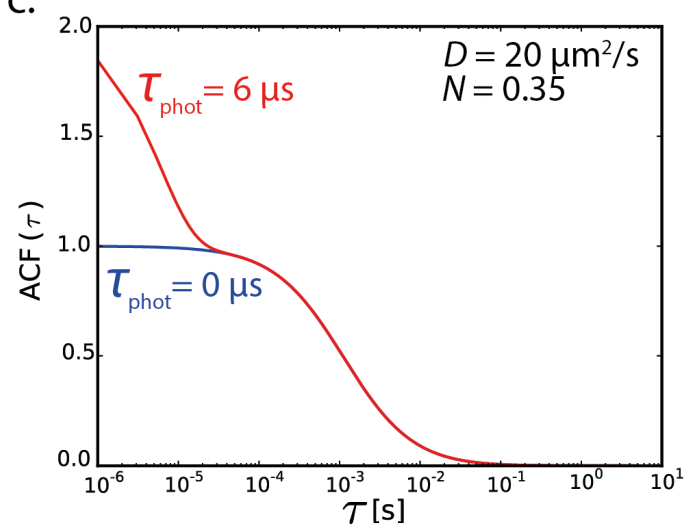

b.

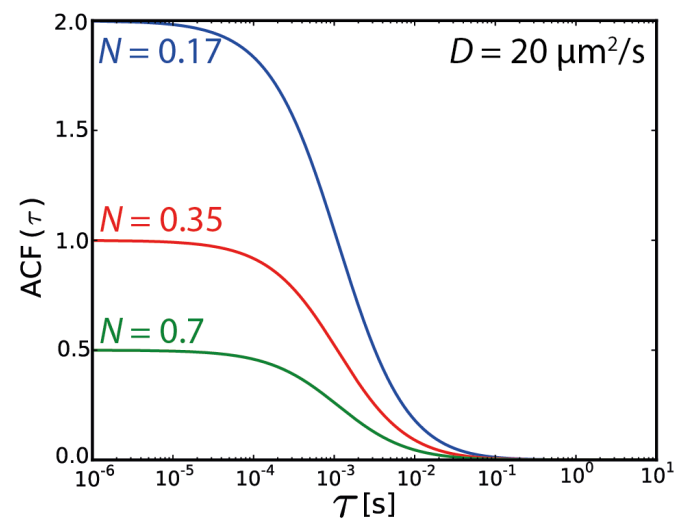

d.

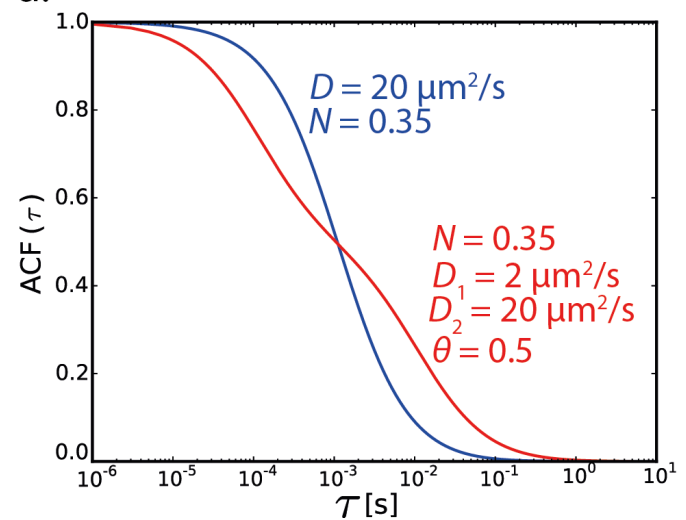

Figure 2.3: Example of FCS curves for different parameters. (a) Increasing the diffusion coefficients translates the curve towards shorter times (green to blue). (b) Increasing the concentration (or the number of molecules in the observation volume), inversely affects the amplitude. (c) ACF curves with (red) and without (blue) triplet component. (d) ACF curves of just one diffusing component (blue) and two diffusing components (red). 
As $\tau$ increases, $G(\tau)$ decreases towards 0. To measure the diffusion coefficient, a least-squares fit is performed on the measured data. As the diffusion coefficient increases, the correlation function shifts to smaller values of $\tau$, as in Figure 2.3a. Since the correlation curve is inversely proportional to the number of molecules in the volume of excitation, an increase in $N$ is reflected as a decrease of the amplitude of the function (Figure 2.3b). The concentration of the sample can be calculated from the number of molecules in the effective volume:

$$
\langle C\rangle=N / V_{e f f}
$$

where the effective volume is described as $V_{\text {eff }}=\left(\frac{\pi}{2}\right)^{3 / 2} w_{0}^{2} z_{0}$.

It is fundamental to measure the effective volume if a quantitative value of $D$ is the goal of the experiment. In fact, even if $D$ is independent from the instrumentation, $\tau_{D}$ is dependent on the radius of $V_{\text {eff }}$. For this reason, before every measurement the setup is calibrated using a dye with a known diffusion coefficient. The correlation function describes how long a diffusing molecule takes to diffuse out of the excitation volume. In particular, equation $(2.6)$ is limited only to diffusion; however, FCS can be used also to characterize the correlation function in general for any process that produces intensity fluctuations.

For example, FCS can be used to describe the fluctuations caused by changes in photo-dynamics [45], rotational effects [44] or motion caused by convective flow [27]. Instead of expressing the fluctuations only as a function of $\delta C$, another model is used to describe the photo-physical fluctuations and derive the correct autocorrelation function. Under certain conditions, depending on the dye molecule, there could also be changes in the brightness on top of the changes due to diffusion. An example is the excitation of the triplet state during absorption, which causes a dark state that leads to an additional term in the correlation function (Figure 2.3c) described as follows:

$$
G_{\text {phot }}(\tau)=X_{\text {phot }}(\tau) G_{D}(\tau)
$$

$G_{\text {phot }}(\tau)$ is the correlation function that describes the triplet effects. It can be defined as the product between the diffusion correlation function $G_{D}(\tau)$ (the same of equation 2.10 and the triplet component $X_{\text {phot }}(\tau)$, which can be described as:

$$
X_{\text {phot }}(\tau)=1+\frac{\Theta}{1-\Theta} \exp -\left(\tau / \tau_{\text {phot }}\right)
$$

where $\tau_{\text {phot }}=\frac{1}{k_{b}+k_{D}}, k_{D}$ and $k_{b}$ are the transition rates for the dark and the bright state and $\Theta=\frac{k_{D}}{k_{D}+k_{b}}$ is the fraction of molecules in the dark state. If the system is composed of two diffusing components with the same brightness, the $\mathrm{ACF}$ curve can be written as the composition of the two diffusing populations:

$$
G_{2 \mathrm{comp}}(\tau)=\frac{1}{N^{2}}\left(N_{1} G_{D_{1}}(\tau)+N_{2} G_{D_{2}}(\tau)\right),
$$


a.

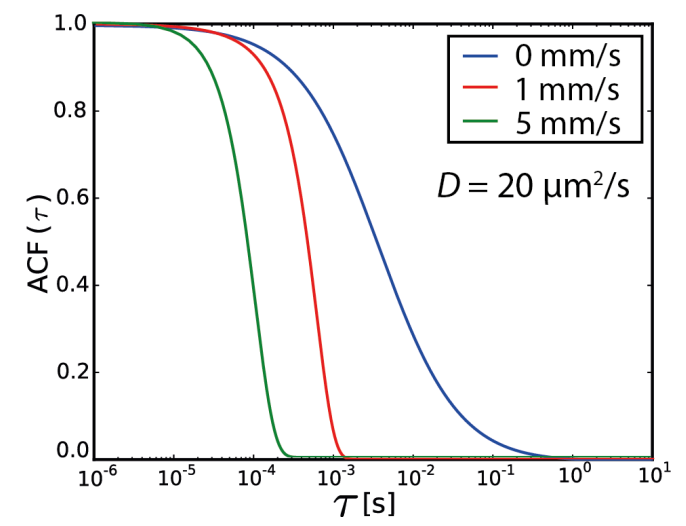

b.

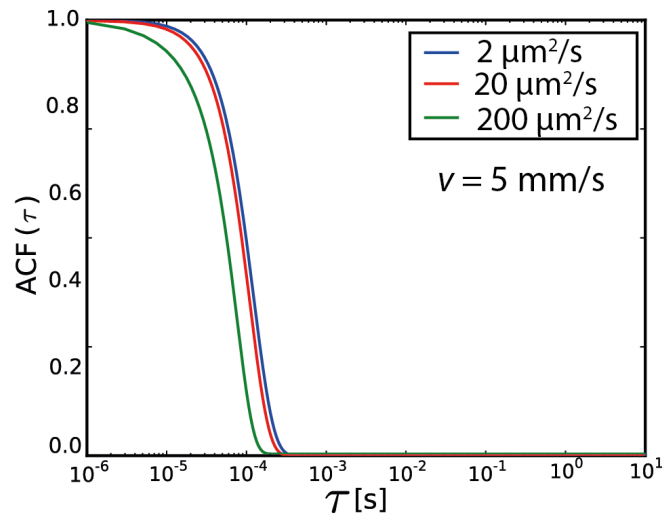

Figure 2.4: ACF with and without convective flow. (a) The ACF curves are calculated with the same diffusion coefficient $\left(\mathrm{D}=20 \mathrm{\mu m}^{2} / \mathrm{s}\right)$ and different velocities. Note how the curves get steeper with increasing flow velocity. (b) ACF curve with the same velocity $\left(5 \mathrm{~mm} / \mathrm{s}, \tau_{F}=\right.$ $66 \mu \mathrm{s})$ but different diffusion coefficients. When the diffusion coefficients are too small compared to the flow velocity, diffusion does not influence the curve anymore. In this image, in the case of $D=2 \mathrm{\mu m}^{2} / \mathrm{s}$ and $D=20 \mathrm{\mu m}^{2} / \mathrm{s}, \tau_{D}$ is too big compare to $\tau_{F}$, thus the two ACFs cannot be distinguished anymore. However, for $200 \mathrm{\mu m}^{2} / \mathrm{s} \tau_{D}$ is actually smaller than $\tau_{F}$, thus diffusion can still be measured.

where $N_{1}$ and $N_{2}$ are the numbers of the diffusing fluorophores, and $N$ is the total number of fluorophores. $G_{D_{1}}(\tau)$ and $G_{D_{2}}(\tau)$ represent only the diffusion part of the correlation function (equation (2.10) for the two components. Figure $2.3 \mathrm{~d}$ is an example of a two component correlation function. With two detectors on the setup, it is possible to cross-correlate the signal from the two channels. If the emission filter is the same for both detectors, the signal-to-noise ratio increases and the effects caused by the detector artifacts (afterpulsing and deadtime) decrease [47. If there are two different emission filters on the detectors, then the cross-correlation function $(\mathrm{CCF})$ can be used to measure for example dynamics in binding experiments: if two populations are labeled with two different dyes, then the cross-correlation signal will be measurable only during a binding event, thus when the photons are detected in both channels at the same time [6].

If the emitting molecules move with an active velocity $v$, the ACF gains an additional component that describes how the combination between convective flow and Brownian motion influences the correlation time:

$$
G(\tau)_{\text {flow }}=G_{D}(\tau) \cdot \exp \left(-\left(\frac{\tau}{\tau_{\mathrm{F}}}\right)^{2} \cdot\left(1+\frac{\tau}{\tau_{D}}\right)^{2}\right),
$$

Here $\tau_{\mathrm{F}}=\frac{w_{0}}{v}$ is the time decay due to the convective flow. If $v$ is too high $\left(\tau_{D}>>\tau_{\mathrm{F}}\right)$ then the contribution of diffusion becomes negligible and this technique cannot be used to measure diffusion (as in Figure 2.4 b) but it is employed to characterize, for example, velocity in microfluidic devices [48]. The effect of the convective flow on the ACF curve is a steepening of the curve due to the extra 
component, as shown in Figure 2.4a.

Since FCS is based on diffusion, it can in principle be also used to measure the molecular weight (MW). If the molecule is spherical, the Stokes-Einstein equation can be used to retrieve the radius of the molecule:

$$
D=\frac{k_{B} T}{6 \pi \eta R}
$$

where $k_{B}$ is the Boltzmann constant, $T$ the temperature, $\eta$ is the viscosity of the solvent and $R$ the hydrodynamic radius. The radius is related to the MW through the specific gravity $\bar{\nu}$ and the volume:

$$
\begin{gathered}
V=M W \bar{\nu}=\frac{4}{3} \pi R^{3}, \\
\Rightarrow R=\left(\frac{3(M W) \bar{\nu}}{4 \pi}\right)^{1 / 3} .
\end{gathered}
$$

The equations show that the radius and the diffusion coefficient are weakly dependent on the MW, in fact an increase of 10-fold in MW corresponds only to a 2.15 -fold increase in $D$. The formation of a dimer from two monomers leads to an increase in D only by a factor of 1.26 or $26 \%$ [6], which could be difficult to measure with FCS. Therefore, if the aim of the experiment is to measure the aggregation of two single molecules forming a dimer, diffusion-based FCS is not the best choice but it can be easily combined with more sensitive techniques as FRET or PCH. The concept of fluorescence correlation spectroscopy was further developed and applied also directly to images from scanning microscopes, confocal or multi-photon microscopes, where it is possible to correlate the signal of each single pixel in time and space [49]. Techniques such as raster image correlation spectroscopy (RICS) or spatial image cross-correlation spectroscopy (ICCS) are applied to study biological systems, as cells or tissue, with high throughput [37, 38 .

\subsubsection{Photon counting histogram}

An alternative approach to study fluorescence fluctuations is called Photon Counting Histogram (PCH). Suppose to have a sample containing two types of molecules with the same diffusion coefficient but a different number of dye molecules attached. In this case it would be impossible to distinguish the two populations based on FCS only. However, independently by their diffusion behaviour the two types of molecules would emit different fluorescence intensities, lower for the dimmer molecule and higher for the brighter molecule. Therefore, if a histogram of the number of appearances of high and low intensity fluctuation is calculated, it is possible to distinguish the two molecules as represented in Figure 2.5. The figure shows the basic idea of $\mathrm{PCH}$; instead of correlating the intensity fluctuations in 
a.
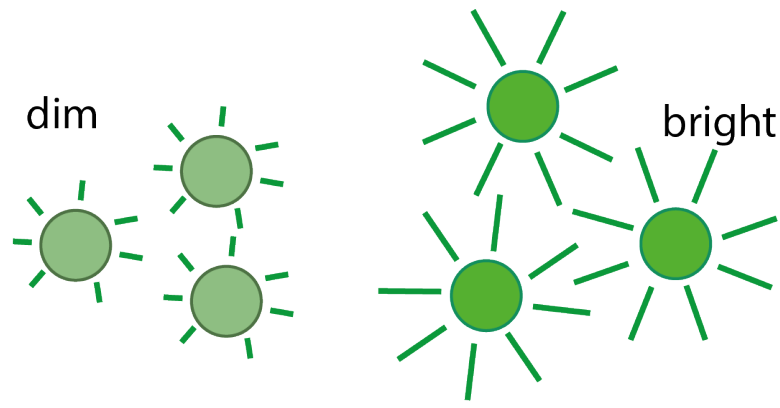

b.

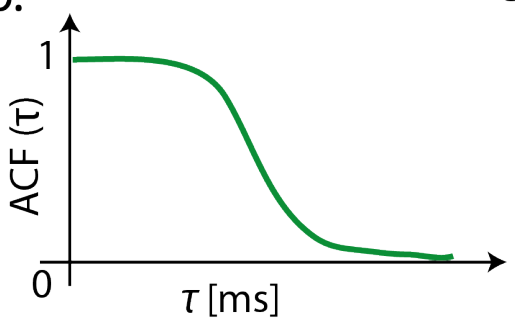

C.

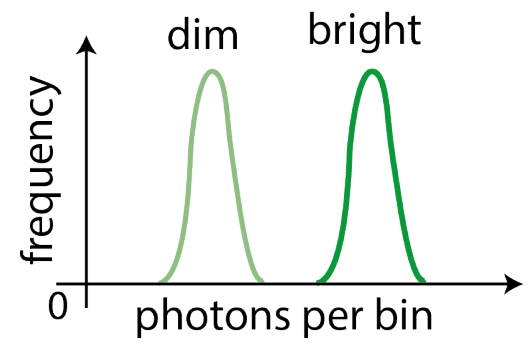

Figure 2.5: Comparison between FCS and PCH for two populations with different brightness and same diffusion coefficient. (a) Two populations of fluorescent molecules with different $B$ are diffusing with the same $D$. (b) Based only on the ACF, distinguish the two populations is impossible. (c) If a histogram of the frequency of the photon counts per time bin are calculated, the two populations can be discriminated by their brightness. Figure inspired by [6].

time, a histogram of fluorescence intensities is created. The detailed mathematical derivation can be found in the Appendix A.3. The first step to analytically illustrate the distribution of intensities is Mandel's formula, which describes, for the semiclassical case, where the light is modeled as an electromagnetic wave and the atom is described according to quantum mechanics, the photon counting statistics measured by the detector [46]:

$$
p(k, t, T)=\int_{0}^{\infty} \frac{\left(\eta_{W} W(t)\right)^{k} e^{-\eta_{W} W(t)}}{k !} p(W(t)) d W(t),
$$

Here $p(k, t, T)$, the probability of observing $k$ photoelectrons at time $t$, depends on the detection efficiency $\eta_{W}$, the integration time $T$ and the energy distribution $p(W(t))$. $W(t)$ represents the light energy falling on the detector surface. It is given by the light intensity integrated over the area of the detector $A$ during the integration time $T$ :

$$
W(t)=\int_{t}^{t+T} \int_{A} I_{D}(r, t) d A d t,
$$

Equation 2.18 is mathematically a Poissonian distribution with two sources of randomness. The first one is a form of noise known as shot noise and it cannot be eliminated. If the light intensity is constant and described as $p(W)=\delta(W-\bar{W})$, 
the photon count distribution can be written as:

$$
\operatorname{Poi}(k,\langle k\rangle)=\frac{\left(\eta_{W} W\right)^{k} e^{-\eta_{W} W}}{k !},
$$

The second source of randomness is caused by the fluctuations of light intensity on the detector, described by $p(W)$. Any source of noise will cause an additional broadening of the photon count distribution. The PCH is indeed described by a super-Poissonian distribution, where the variance is larger than the mean. The fluctuations of light intensity are dependent on the integration time $T$. In the limit of $T \rightarrow \infty$ the fluctuations average out, $p(W)$ approaches a delta function and the $\mathrm{PCH}$ narrows down to a Poissonian distribution. In the other limit, $T \rightarrow 0$, the fluctuations perfectly track the light intensity $(I)$ in time. In this case, the probability distribution of energy and intensity are proportional to each other: $p(W)=p(I) \Delta t$. It is fundamental to choose an integration time shorter than the fluctuation time scale of the studied process $\left(T<\tau_{\text {fluc }}\right)$ to be able to approximate $p(W)$ with the intensity. For simplicity, it is also assumed that the detector area $A$ is so small that the intensity is constant on the detector surface. If so, we can rewrite 2.18 as:

$$
p(k)=\int_{0}^{\infty} \frac{\left(\eta_{I} I_{D}\right)^{k} e^{-\eta_{I} I_{D}}}{k !} p\left(I_{D}\right) d I_{D}=\int_{0}^{\infty} \operatorname{Poi}\left(k, \eta_{I} I_{D}\right) p\left(I_{D}\right) d I_{D},
$$

It was assumed here that the statistical proprieties of the intensity fluctuations are not time dependent, thus the photon count distribution is also time independent. The new detector efficiency $\eta_{I}$ is dependent on the light intensity upon the detector $I_{D}$ and takes into account the integration time $T\left(\eta_{I}=T \eta_{W}\right)$. The emitted fluorescence intensity by a fluorophore at a position $\overrightarrow{r_{0}}$ (defined as the PSF center), which is measured on the detector plane, can be written as:

$$
I_{D}=I_{0} \beta P S F\left(\overrightarrow{r_{0}}\right),
$$

where the constant $\beta$ includes the excitation probability, the fluorescence quantum yield and all the set-up related factor such as the transmittance of light after the optics. The probability of fluorescence intensity on the detector can now be written as:

$$
p\left(I_{D}\right)=\int \delta\left(I_{D}-I_{0} \beta P S F(\vec{r})\right) p(\vec{r}) d \vec{r}
$$

Here $p(\vec{r})$ is the probability distribution for the position of the fluorophore. In a first approach it is assumed that there is a single emitting particle inside a reference volume $V_{0}$. Because it is equiprobable to find the particle in any position inside $V_{0}$, the probability $p(\vec{r})$ is given by $1 / V_{0}$ if the particle is in $V_{0}$, or 0 otherwise. 
The molecular brightness is defined as $b=I_{0} \beta \eta_{I}=I_{0} \beta \eta_{W} T$; it represents the intensity measured on the detector emitted from a particle in the center of the PSF during one integration time. It is dependent on the detection optics and the integration time. Combining $b$ with equation 2.21 and equation 2.23 it is possible to write the $\mathrm{PCH}$ for a single particle in a closed volume $V_{0}$ as:

$$
p^{(1)}\left(k, V_{0}, b\right)=\frac{1}{V_{0}} \int_{V_{0}} \operatorname{Poi}(k, b P S F(\vec{r})) d \vec{r},
$$

The reference volume $V_{0}$ is chosen so that it contains the PSF. There is no photon excited outside $V_{0}$, thus extending the integration limits to infinity does not change $p^{(1)}$, unless $k=0$. The probability of receiving 0 photon counts is then calculated by normalizing the probability distribution: $p^{(1)}\left(0, V_{0}, b\right)=$ $1-\sum_{k=1}^{\infty} p^{(1)}\left(k, V_{0}, b\right)$. Since the analytic form of the PSF is known (equation 2.9 for the confocal case), it is possible to expand the integral from $V_{0}$ to infinity:

$$
p^{(1)}\left(k, V_{0}, b\right)=\frac{1}{V_{0}} \frac{\pi \omega_{0}^{2} z_{0}}{k !} \int_{0}^{\infty} \gamma\left(k, b e^{-2 x^{2}}\right) d x, \text { for } k>0
$$

where $\gamma$ represents the incomplete $\gamma$-function. Equation 2.25 represents the case for only one emitting particle. If there are $N$ particles in $V_{0}$ with identical brightness, at position $\vec{r}_{n}$, then the $\mathrm{PCH}$ becomes:

$$
p^{(N)}\left(k, V_{0}, b\right)=\int \cdots \int \operatorname{Poi}\left(k, b \sum_{i=1}^{N} \operatorname{PSF}\left(\vec{r}_{i}\right)\right) p(\vec{r})_{1} \ldots p\left(\vec{r}_{N}\right) d \vec{r}_{1} \ldots d\left(\vec{r}_{n}\right),
$$

If it is assumed that the particles are non-interacting, the $N$ variables can be treated as statistically independent. The probability distribution of the sum of statistical independent variables can be then expressed as the convolution of $N$ individual probability distribution functions [50].

$$
p^{(N)}\left(k, V_{0}, b\right)=\left(p^{(1)} \otimes \cdots \otimes p^{(1)}\right)\left(k, V_{0}, b\right),
$$

This equation represents the photon count distribution for $N$ identical particles in a closed reference volume $V_{0}$. If an open system is considered, with a reference volume $V_{0}$ much smaller then the open system, the number of fluctuating particles in $V_{0}$ is governed by Poisson statistics:

$$
p_{\#}(N)=\operatorname{Poi}(N, \bar{N}),
$$

where $N$ is the actual number of molecules in the reference volume and $\bar{N}$ is the average number of molecules. $\bar{N}$ can be calculated from the concentration of the sample in $V_{0}, \bar{N}=C V_{0} N_{A}$ ( $N_{A}$ is Avogadro's number). The final step in calculating the $\mathrm{PCH}$ for an open system is averaging the individual probability 
function for $N$ particles weighted by the Poissonian probability of observing $\mathrm{N}$ particles:

$$
\Pi\left(k, \bar{N}_{P S F}, b\right)=\left\langle p^{(N)}\left(k, V_{0}, b\right)\right\rangle_{N}=\sum_{N=0}^{\infty} p^{(N)}\left(k, V_{0}, b\right) p_{\#}(N),
$$

Since the $\mathrm{PCH}$ is independent of a physical volume, instead of calculating the distribution for a reference volume, the distribution is calculated using $\bar{N}_{P S F}$, that is the average number of molecule in the PSF. Changing $\bar{N}$ with $\bar{N}_{P S F}$ is justified because $C=\bar{N}_{P S F} /\left(V_{P S F} N_{A}\right)=\bar{N} / V_{0} N_{A}$. The average number of photon counts for an open system can be later calculated from $\Pi\left(k, \bar{N}_{P S F}, b\right)$ as:

$$
\langle k\rangle=b \bar{N}_{P S F},
$$

If the sample is composed by two species $N_{1}$ and $N_{2}$ with two different brightnesses $b_{1}$ and $b_{2}$, the PCH is given by:

$$
\begin{array}{r}
p^{\left(N_{1}, N_{2}\right)}\left(k, V_{0}, b_{1}, b_{2}\right)=\int \cdots \int p\left(\vec{r}_{i}\right) d\left(\vec{r}_{i}\right) \ldots p\left(\vec{r}_{j}\right) d\left(\vec{r}_{j}\right) \\
\operatorname{Poi}\left(k, b_{1} \sum_{i=1}^{N_{1}} \operatorname{PSF}\left(\vec{r}_{i}\right)+b_{2} \sum_{j=1}^{N_{2}} \operatorname{PSF}\left(\vec{r}_{j}\right)\right),
\end{array}
$$

If the two species are independent the convolution trick can be applied again:

$$
\Pi\left(k, \bar{N}_{1}, \bar{N}_{2}, b_{1}, b_{2}\right)=\Pi\left(k, \bar{N}_{1}, b_{1}\right) \otimes \Pi\left(k, \bar{N}_{2}, b_{2}\right),
$$

For $N$ species the PCH will be described by the $N$-times convolution of the single species distribution. It is important to notice that in the $\mathrm{PCH}$ model there is no explicit dependence on the size of the PSF but only on the geometrical shape. However if the shape of the experimental PSF is not perfect, an additional correction factor has to be introduced [51]. The deviations from the 3D Gaussian model, used in the OPE case, are critical especially for bright particles in the region away from the focal point. In [51] the authors approach this problem by introducing the factors $F_{j}$ defined as the the relative difference between the integral of the $j$-th power of the experimental volume and that of its 3D Gaussian approximation. In most cases, the first order correction alone is sufficient to correct the deviations. The correction is applied to the one-photon distribution and then the calculation of the PCH for $N$ molecules is carried on as explained before.

$$
p^{(1)}\left(k, V_{0}, b\right)=\frac{1}{(1+F)^{2}} p_{G}^{(1)}\left(k, V_{0}, b\right) \text { for } k>1
$$




$$
p^{(1)}\left(1, V_{0}, b\right)=\frac{1}{(1+F)^{2}}\left[p_{G}^{(1)}\left(1, V_{0}, b\right)+\frac{b F}{2 \sqrt{2} V_{0}}\right] \text { for } k=1
$$

Here $p_{G}^{(1)}\left(k, V_{0}, b\right)$ is the count distribution for a 3D Gaussian model (eq. 2.25 ) and $F$ describes the fraction of detected photons from the deviated part of the $\mathrm{PSF}$. The PCH can also be affected by detector artifacts caused by the nonideality of the photodetectors [52]. The two main effects are the dead-time and the afterpulse. Afterpulses are spurious pulses generated by the detector after having detected a real event. Usually afterpulses increase the counts for high count rates. The dead-time is a short fixed time in which the detector is "blind" after the registration of a photon. It is particularly important when the concentration of the sample is high, because at high count rates many photons can be lost during the dead-time. The effect on the PCH in this case is a narrowing of the distribution at the higher channels. In [52] the theory behind PCH is developed to take into account also these two effects. Regarding the dead-time, it is demonstrated that the count distribution affected by dead-time can be written as the sum of ideal $\mathrm{PCH}$ distributions with a reduced brightness.

$$
\Pi(k, b, \bar{N}, \delta)=\sum_{j=0}^{k} \Pi(j, b(1-k \delta), \bar{N})-\sum_{j=0}^{k-1} \Pi(j, b(1-(k-1) \delta), \bar{N}),
$$

where $\delta=\tau_{D t} / T$ is the parameter that represents the dead-time effect with $\tau_{D t}$ being the dead-time of the used detector (usually it is around $50 \mu \mathrm{s}$ ). This model analytically describes the dead-time effects and it is normally employed for all the PCH analysis when $N>1$. There is no analytical model to correct for afterpulses. Since the probability of afterpulsing is usually very low, especially at low count rates, it was not considered in the model used for this work.

$\mathrm{PCH}$ can also be applied in combination with microfluidics. As long as undersampling is avoided, flow does not affect the distribution of photons [53]. Thus, as long as the integration time $T$, called also sampling time, is faster than the time scale of the considered fluorescence fluctuations, the shape of $\mathrm{PCH}$ is not dependent on the flow, while the autocorrelation function is affected by it (see the previous section). To treat fluorescence fluctuations data in a statistical way it is fundamental that the process studied is stationary. A stationary process is a process whose distribution function does not change when shifted in time or space. Laminar flow acts like a translation in space, and the fluctuations are in fact caused by a stationary process. However, to faithfully tracks the fluorescence intensity over time, which allow us to switch between energy and intensity in the $\mathrm{PCH}$ derivation (equation 2.21), we have to take into consideration that the fluctuation rate increases when the flow velocity is increased [53. If the velocity is too high and the sampling time $T$ is too short to track the intensity, then the $\mathrm{PCH}$ 
model is not valid anymore. PCH is often used in combination with FCS to characterize the observation volume [59], to study receptor-receptor interactions in cells [60] and to study protein folding or unfolding [61] or changing conformations in molecules with single molecule resolution [62]. FCS and PCH are complementary techniques. For example, in [60] the authors use FCS to map protein diffusivity in cell membranes. However, since diffusion times scale with the cubic root of the mass, it is difficult to measure protein aggregation with FCS. For this reason $\mathrm{PCH}$ is used to then measure the oligomerations of proteins at the uPAR receptors, since it can measure the brightness of the emitting molecules. $\mathrm{PCH}$ was also used to study cytoskeleton filaments, in particular actin polymerization [63. The authors could measure the size distribution of actin oligomers during polymerization in vitro, measuring again the brightness and the label stoichiometry of the sample.

\subsection{Microfluidics}

This chapter will introduce the concepts of microfluidics for studying biological samples. Microfluidic techniques are popular tools which can be employed to manipulate, in a controlled manner, small amounts of fluids inside micrometer-sized channels. Apart from being a very controlled method, microfluidics also allows us to utilize little amounts of sample, helping to decrease reactions time [69]. Thus, microfluidics is engaged to measure fast protein kinetics, such as unfolding and folding [74], using a continuous flow scheme, since dead time in microfluidic mixers is drastically reduced. To decrease the mixing times, hydrodynamic focusing is applied. The protein stream is narrowed down to decrease the time needed for the second component to diffuse completely inside the focused stream [58]. A basic microfluidic device used for hydrodynamic focusing has just three inlets, in this case the diffusive mixing starts before the central stream is completely focused. However, to have a better control on the starting point of the studied process, the mixing should be as fast as possible, therefore it should start only when the central stream is already focused to the smallest stream possible. To delay the diffusion of the side inlets solution into the central stream, a pair of diagonal inlets are inserted between the central and the side inlets [75]. A buffer solution with a slow flow rate is pumped through the diagonal inlets, "moving" the starting mixing point down along the outlet channel where the central stream is already focused, as shown in Figure 4.5 . Applications of microfluidics are very broad in biophysics [13] since, apart from being employed as tool to reliably control small scale systems, microfluidic devices have other advantages such as low cost of manufacturing, high throughput and short reaction times [13]. Here, in particular continuous flow microfluidics is applied to track molecular interactions and protein assembly over time [14, 15, 16]. 


\subsubsection{Flow at low Reynolds number}

The Navier-Stokes equation is used to describe fluid dynamics in general [70, 71]:

$$
\rho(\vec{r}, t)\left(\frac{\partial \vec{v}}{\partial t}+(\vec{v} \cdot \vec{\nabla}) \vec{v}\right)=-\vec{\nabla} p+\eta \nabla^{2} \vec{v}+\vec{F}_{\text {ext }} .
$$

Here $\vec{v}$ is the velocity of the fluid, $p$ is the pressure, $\eta$ the viscosity and $\vec{F}_{\text {ext }}$ represents all the possible external forces applied to the system. This equation, in its general form (non-linear, second order partial differential equation), is not at all easy to handle, however it can be simplified under certain conditions. If the fluid studied is incompressible, as for example water, the density $\rho(\vec{r}, t)$ is constant and it can be simplified to $\rho$. The left hand side of the equation represents the inertial forces, with the terms of fluid acceleration and convection. The terms on the right hand side are related to pressure, viscous force and external forces [71]. A dimensionless number can be defined to compare the two sides of equation 2.35. This quantity is called the Reynolds number $(R e)$ and it can be considered as the ratio between inertial and viscous forces:

$$
R e=\frac{\rho V_{0} L_{0}}{\eta},
$$

where $V_{0}$ and $L_{0}$ are the characteristic velocity and the characteristic length scale of the system respectively. When $R e$ is small, the inertial component is negligible with respect to the viscous component. In this case, the flow is in a laminar regime. Under this condition the flow is stationary and time reversible, there is no turbulence. Equation 2.35 can be simplified to the Stokes equation:

$$
\vec{\nabla} p=\eta \nabla^{2} \vec{v}
$$

Equation 2.37 is valid in laminar flow when there are no external forces. For $R e>2300$ inertial forces dominate and the flow starts to show signs of turbulence [72]. In microfluidics, usually the length scales are in the order of micrometers; for water in a channel with a width of $100 \mu \mathrm{m}$, flowing with a velocity of $1 \mathrm{~mm} / \mathrm{s}$, $R e$ is in the order of 0.1 . In this case, since $R e$ is smaller than the critical value, and we can assume laminar flow.

\subsubsection{Flow profile at low Reynolds number}

When the flow is laminar (low Re) the flow profile can be described, in certain conditions, by the Poiseuille equation that analytically solves equation 2.35. The fluid has to be incompressible and Newtonian. In Newtonian fluids, the viscosity is constant and not dependent on the shear stress arising from the flow. The shear stress in Newtonian fluids is linearly proportional to the shear rate through a constant, the dynamic viscosity $\eta$. A "no-slip" condition at the boundary is 
chosen to solve the Poisseuille equation (the velocity is zero at the channel walls). The solution for the flow rate $Q$ in a channel of circular cross section is:

$$
Q=\frac{\Delta p}{8 \eta L} \pi R^{4}
$$

and for the velocity:

$$
\left.v(\vec{r})=\frac{\Delta p R^{2}}{4 \eta L}\left(1-(\vec{r} / R)^{2}\right)\right),
$$

where $R$ is the radius of the channel, $\Delta p$ is the pressure difference between inlet and outlet, $\eta$ is the viscosity, $L$ is length of the channel segment considered and $\vec{r}$ is the axial coordinate with the origin at the center of the pipe. For a rectangular cross section the solutions are more complicated, however the velocity profile still depends quadratically on $\vec{r}$. This means that the velocity in the microfluidic devices used in this work, which all have rectangular cross section, has a parabolic profile in $y$ and $z$ ( $x$ is the direction of the flow). The complete derivation can be found in [71].

\subsubsection{Mass transport in microfluidic devices}

Since microfluidic devices are employed in this work as mixers, it is important to consider how mass is transported within the device. Mainly two processes occur, the active transport due to convection and the passive transport due to diffusion. They can be both described by the diffusion-convection equation:

$$
\frac{\partial C}{\partial t}=\nabla(D \nabla C)-\nabla(\vec{v} C)+S
$$

here, $C$ is the concentration of the transported sample, $D$ is its diffusion coefficient and $S$ is a coefficient denoting any other additional sources or sinks of $C$. The velocity field, $\vec{v}$, is calculated from equation 2.35. When the diffusion coefficient is small, the convection component of the equation dominates. The diffusion coefficient can be expressed for a generic particle, following Einstein and Smoluchwski relation, as:

$$
D=\mu k_{B} T
$$

where $\mu$ is the mobility of the particle, $k_{B}$ is Boltzmann constant and $T$ the temperature. When the flow studied is laminar, the mobility can be written as the inverse of the drag coefficient $\left(c_{d}\right), \mu=c_{d}^{-1}$. If the particles flowing are small spheres with a radius $R$, then:

$$
c_{d}=6 \pi \eta R
$$

The diffusion coefficient can be easily calculated using equation 2.16. In the experiment, where assembly of vimentin is studied (Chapter 4.1), these equations are employed to check when the salt concentration is uniform in the protein 
stream. The full characterization of these processes is realized using Finite Elements Methods simulations (FEM) that will be explained in Chapter 3.5. As for Navier-Stokes equation, where a dimensionless number is defined to quantify the ratio between viscous and inertia forces $(R e)$, here the Péclet number $(P e)$ quantifies the ratio between diffusion mixing and convective mixing [73. If two solutions are brought in contact in a channel junction, the time until mixing by diffusion only is completed, $\tau_{D m i x}$, is inversely proportional to D:

$$
\tau_{\text {Dmix }} \approx \frac{\omega_{c}^{2}}{D}
$$

where $\omega_{c}$ is the channel width. During the same time $\tau_{\text {Dmix }}$, the solution will flow down the channel with a velocity $v$ for a length $s$ :

$$
s=v \tau_{D m i x} \approx \frac{\omega_{c}^{2} v}{D},
$$

Dividing $s$ by the channel width we find $P e$ :

$$
P e=\frac{\omega_{c} v}{D} .
$$

The Péclet number quantifies the distance along the channel, in terms of the channel width, after which mixing of two solutions is completed. If $P e$ is small, diffusion dominates the transport in the device. If $P e$ is big, the solute follows the flow lines; in the limit of $P e \rightarrow \infty$ there will be no mixing in the laminar flow regime. In our case, diffusive mixing is relevant for the mixing of monovalent ions in the protein stream during the assembly measurement. For a protein with a diffusion coefficient in the order of $10^{-11}$, moving with a velocity of $1 \mathrm{~mm} / \mathrm{s}$ and $\omega=100 \mu \mathrm{m}, P e$ is around $10^{4}$, while for an ion with a diffusion coefficient in the range of $10^{-9}, P e$ is around 100 . In this case the ion will diffuse along the width of the channel faster than the proteins. Thus, due to the laminarity of the flow in continues flow microfluidic devices, mixing becomes purely a diffusion process. Mixing time scales can be approximated with the diffusion times of the ions. In this case, the mixing has a low efficiency, which can be exploited to create concentration gradients [77]. When a total mixing is the final goal of the experiments the geometry of the device is usually adapted and improve to increase the mixing of the streams [76]. In Section 4.1.4 the initiation of vimentin assembly is achieved by addition via diffusion of ions into the weakly diffusive vimentin stream. The mixing times are reduced by hydrodynamic focusing the protein stream, thus the length required for mixing is reduced [163]. The focusing can be controlled changing the flow rates applied [163], therefore also the mixing times can be easily regulated tuning the flow rates. This mixing approach, where small reactants are mixed with slowly diffusive molecules, has been applied also to study for examples controlled assembly of spider silk proteins [64, collagen self-assembly under a pH gradient [164], ion induced RNA folding [65], kinetics of protein folding [66] or kinetics of induced hydrogelation and nanofibrils [67]. 


\subsection{Biological background}

In this section the biological components studied in the thesis will be introduced. In the first part of the section, there will be an introduction to vimentin, a component of the cytoskeleton in the family of intermediate filaments. In particular, vimentin assembly will be described, as in Chapter 4.1 the early temporal steps of vimentin assembly are studied. In the second part of this section, synapses and synaptic proteins will be introduced. In one project the interaction between synaptic vesicles and synaptic proteins is studied in cellulo and in vitro; this project is in collaboration with Sofiia Reshetniak and Prof. Silvio Rizzoli from the Institute for Neuro- and Sensory Physiology in University Medical Center of Göttingen. In the other project, a microfluidic chamber is build to help studying in a controlled fashion neurotrasmitters uptake by synaptic vesicles; this project is a collaboration with Helena Maria (Linda) Olsthoorn and Prof. Reinhard Jahn from the Laboratory of Neurobiology of the Max Plank Institute for Biophysical Chemistry in Göttingen.

\subsubsection{Intermediate filaments}

In the human body around 200 different types of cells are present 78. Despite their huge diversity, from long and branched nerve cells, to highly motile fibroblasts, all different types of cells have the same underlying architecture, the cytoskeleton. The cytoskeleton is considered playing the major role in keeping the cell shape and in providing cells with mechanical resistance [78]. It is composed by a dynamic network of filaments and it is involved in cell migration and in some cell signaling pathways [79, 95]. The cytoskeleton is composed by three types of filaments: microtubules, microfilaments (or actin filaments) and intermediate filaments, as shown in Figure 2.6.

Microtubules are hollow cylinders formed by monomers of alpha and beta tubulin, they are the thickest filaments ( $23 \mathrm{~nm}$ of diameter) in the cytoskeleton. Microtubules are considered the "highways" of cells, as they are used by kinesin proteins for intracellular transport. Microtubules are also involved in the correct positioning of cell organells and in cell division, where they are part of the mitotic spindle. Microfilaments are linear polymers (diameter around $7 \mathrm{~nm}$ ) composed by $\alpha$-actin monomers, in muscle cells, or $\beta$-actin or $\gamma$-actin, for all the other cell types. They are important in maintaining the proper cell shape, in cell signaling, division and in cell motility [79].

Intermediate filaments, IFs, are the least well studied cytoskeletal filaments. They have a diameter in between microtubules and microfilaments, around 10 nm. They help cells to resist mechanical stresses and conformational changes. While microtubules and microfilaments are conserved between all cell types, different IFs are expressed in different cell types. In fact there are at least 70 genes in humans that are known for encoding different types of IFs. In mammals, IFs 
a.

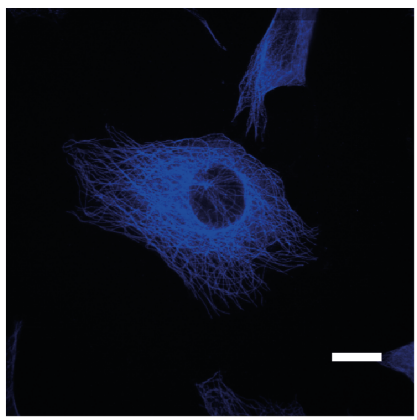

C.

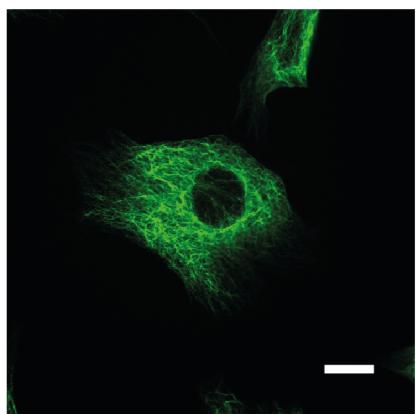

b.

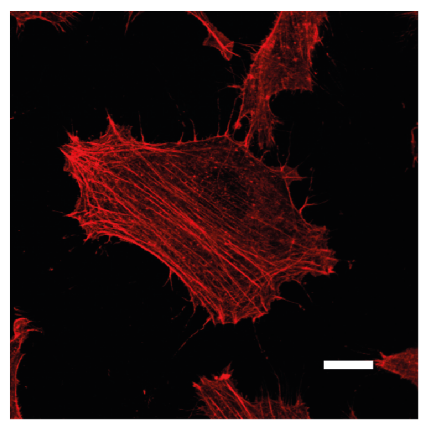

d.

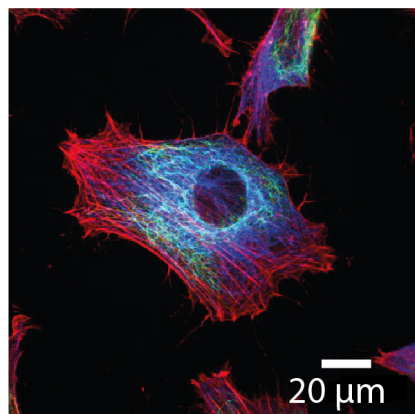

Figure 2.6: Fluorescence confocal images of cytoskeleton filaments, microtubules (a), actin (b), vimentin (c), in 3T3 mouse fibroblast cell. The overlay of the three channels is shown in (d). The scale bar is $20 \mu \mathrm{m}$ in all pictures. Images courtesy of Dr. Ulrike Rölleke.

can be grouped into five categories [81] based on their sequence homology [80, as shown in table 2.1. The division into 5 categories reflects also different biological origins and functions in cells. For example keratins are typically found in epithelial cells, vimentin is usually found in mesenchymal cells and neurons involve neurofilaments. Lamins are found around the nuclei [82] in higher organisms, forming an inner envelope for the nucleus.

Despite the different classes, all IFs share similar secondary structure, including helical rod domains and unstructured heads and tails. The central $\alpha$-helical rod domain is conserved in size in all the IFs apart from the lamins. It is composed by three coils (1A, 1B and 2) connected by linkers (L1 and L12), as shown in Figure 2.7a. They also have a similar hierarchical process for assembling and this will be the main topic of the following subsection.

\section{Assembly of vimentin}

While microtubules and actin assemble into filaments starting from globular monomers in a polar fashion [79, 95], IFs follow a hierarchical assembly starting from rod shaped monomers. In particular, we will focus on the assembly process of vimentin, an IF from the third class of IFs, typically present in mesenchymal cells. 


\begin{tabular}{lcr}
\hline Class & IFs & cell type \\
\hline SHC 1 & acidic keratins & epithelial cells \\
SHC 2 & basic keratins & epithelial cells \\
SHC 3 & e.g. vimentin, & mesenchymal cells, muscle \\
& desmin, GFPA & cells, astrocytes, glia cells \\
SHC 4 & $\alpha$-internexin, neurofilaments & neurons \\
SHC 5 & lamins (type A/C and B) & nucleated cells \\
\hline
\end{tabular}

Table 2.1: SHC (sequence homology class) classes of IFs, examples of the IFs in that class and their occurrence in mammalian cells (adapted from [80]).

A schematic representation of vimentin assembly in vitro is shown in Figure 2.7. The assembly starts from the monomer. Two monomers align laterally to form a coiled-coil dimer and two dimers align, again laterally but in antiparallel mode, to form a half-staggered non-polar tetramer [83, 95]. These steps of assembly happen spontaneously in vitro during the dialysis of the protein from a denaturing agent buffer (e.g. $8 \mathrm{M}$ Urea) into a more physiological buffer (in our case $2 \mathrm{mM}$ phosphate buffer). With available crystal data structures of vimentin dimer, an atomic model of the anti-parallel vimentin tetramer is constructed [121]. These tetramers are formed in a half-staggered fashion, so that the first parts of the rod domains are roughly aligned with each other in the " $\mathrm{A}_{11}$-mode" [119, 121]. In the atomistic model from [121, the $\alpha$-helical L1 domain is aligned with the C-terminal of coil 1B of the second dimer. This alignment is partly promoted by a complementary charge pattern in the rod domain of the two dimers, which helps to maintain the $\mathrm{A}_{11}$-mode [121. While the charge pattern on the rod domain is important, also the head domain of vimentin monomers is fundamental for the tetramer formation [95], as experiments with mutant "headless" vimentin or isolated vimentin rod stay in the dimeric stage even in tetrameric condition buffers [119.

With the addition of monovalent salt (increasing the buffer ionic strength) the assembly proceeds with the formation of unit length filaments (ULF) that finally start to assemble longitudinally forming filaments. The number of monomers in each ULF depends on the IFs considered; in the case of vimentin, 32 monomers are present on average. However mass-per-length measurements showed that the number of sub-units per ULF can vary in filaments of the same type or even inside one single filament [85, 86, 88]. This "polymorphism" was shown to be influenced by the method used to start the assembly [119]. When the monovalent salt solution is added instantaneously to the protein the number of sub-units per cross section along the filament is less uniform than when the filament is formed by dialysis into a salt buffer [119]. In vimentin and some other IFs, there is an additional compaction step, during the filament formation, that decreases the diameter of the filament from $17 \mathrm{~nm}$ to $10 \mathrm{~nm}$. It is important to notice that the 


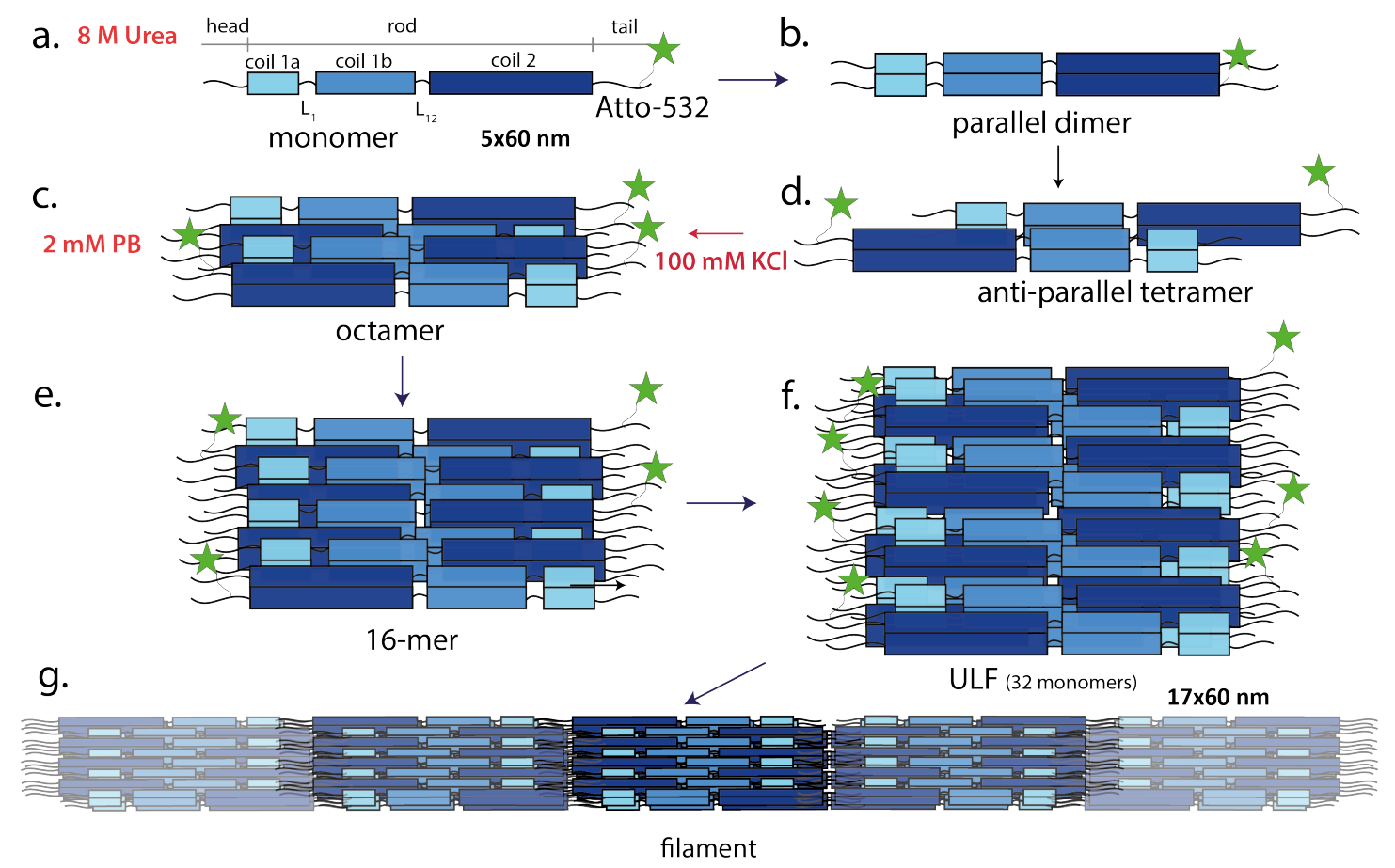

Figure 2.7: The assembly starts from monomer of vimentin (5x60 nm composed by $\alpha$-helices rod domains and unstructured head and tail domains) and continues laterally up to the formation of ULF structures. ULFs starts then to assemble longitudinally forming a filament.

assembly of IFs does not require any additional energy, in contrast to microtubules and actin. Moreover IFs are non-polar, due to the anti-parallel annealing step that forms the tetramers, while both microtubules and microfilaments are polar.

Unusually for proteins, IFs tend to form stable dimers even at high concentration of urea, for example, vimentin forms ordered tetramers already in $5 \mathrm{M}$ urea [119] and at the same urea concentration keratin forms dimers [84]. The assembly dynamics is influenced by temperature, ion type, ion concentration [93], and protein concentration [88, 95]. The assembly dynamics was revealed by electron microscopy (EM) [119, 88, 120] and atomic force microscope (AFM) [89, 91].

IF assembly can be considered as a two step process: the lateral assembly, up to ULF formation, is very fast, within seconds [91], while the elongation of the filaments is a slower process that takes place in minutes. Commonly, vimentin assembly is studied using "static" techniques such as EM or AFM. With these two microscopy methods, the first step of assembly vimentin, the lateral assembly, cannot be measured. In fact, the time needed to prepare the sample to measure, is longer than the ULF assembling time. Vimentin assembly was also monitored using light-scattering methods such as dynamic light scattering (DLS) [87]. DLS is used to measure the first elongation steps of vimentin filaments. For these measurements, as in the case of EM and AFM experiments, the vimentin later 


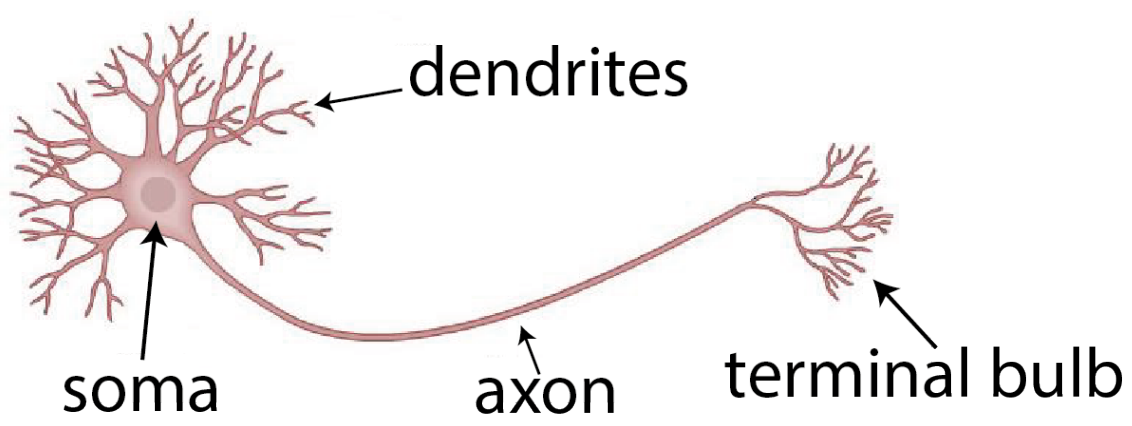

Figure 2.8: Basic illustration of a neuron. Neurons are composed by the soma, or cell body, dendrites and a long axon. At the end of the axon the information are transmitted through the terminal bulb. Adapted from [112].

assembly cannot be accessed, since the sample preparation takes between 10 to 30 seconds [87]. A similar technique, static light scattering (SLS), in combination with a stopped-flow setup, was used to overcome the time limitations and measure the dynamics during the formation of ULF [96]. Due to fast mixing of vimentin and buffer, assembly was measured after just $10 \mathrm{~ms}$ of dead time. The authors confirm that the formation of ULF is very rapid, most of the tetramers assemble into ULFs in the first $100 \mathrm{~ms}$ of assembly. However, in the stopped-flow device chaotic mixing is used to achieve fast mixing, leading to differences in the number of vimentin monomers for single ULFs. As an alternative to the stopped-flow measurements, slow measurement techniques can be use in combination with microfluidics in laminar flow regime. Microfluidic devices are used to mix, this time by diffusion, protein and assembly buffer, in a similar condition to the slow assembly by dialysis. As soon as the assembly starts, measurements can be taken in different positions along the channel, translating the spatial coordinates into time coordinates. To obtain a better time resolution, X-ray scattering was combined with microfluidics [92, 93], also confirming the formation of ULF to be on the time scale of one second [14]. However, X-rays methods are limited by the high protein concentration needed to have good signal. Fluorescence methods, as FCS or $\mathrm{PCH}$, can be used even at very low concentration [53], and the combination with microfluidic devices, decreases the acquisition time for each measurement, making these techniques good candidates to measure the fast assembly of vimentin.

\subsubsection{A brief introduction to synapse}

Neuronal cells, or simply neurons, are the cells dedicated to transport signals between the different parts of the body. They are excitable cells that respond to electric stimuli. They are mainly composed of a cell body, called soma, dendrites and a single long axon, as shown in Figure 2.8. The dendrites and the axons are extensions from the soma, and axons can be up to $1 \mathrm{~m}$ long in humans. Most 


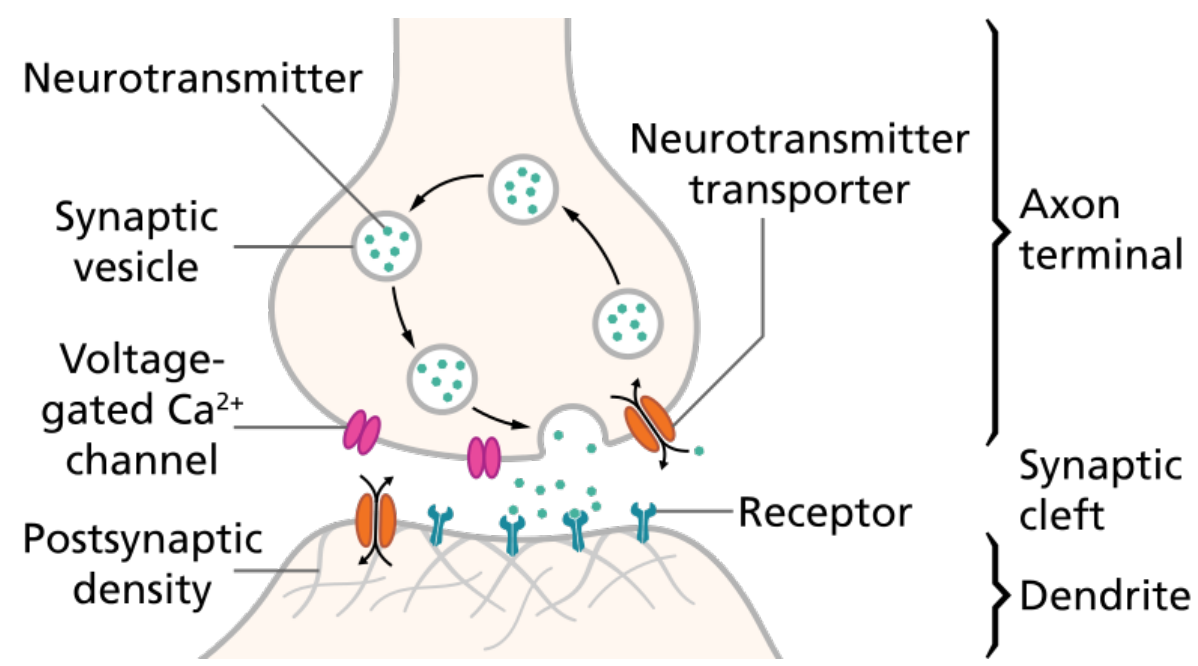

Figure 2.9: Schematic representation of a chemical synapse. An action potential causes the neurotransmitters, transported by the synaptic vesicles, to be released from the axon terminal, or synaptic bouton, into the synaptic cleft, causing an electrical signal in the post synaptic region. Image reprinted under the Creative Commons Attribution (CCBY) license.

of the neurons receive information through the dendrites and send the signal via the axons. The actual information are passed through neurons via synapses. The synaptic bouton transmits the information to the next neuron with chemical neurotransmitters. Neurons may have additional synapses along the length of the axons in addition to synapses in the axons.

There are two types of synapses in humans, electric and chemical [98]. Electric synapses have shorter gap distance between the two neurons, thus can propagate nervous impulses faster. They are mostly found in neural systems where a fast response is fundamental, as defence reflexes. They differ from chemical synapses because they lack gain response to the original signal, but their signal transmission can be bidirectional. Chemical synapses have a larger gap (around $40 \mathrm{~nm}$ ) between the two neurons and transmit information via small neurotransmitter "containers" called synaptic vesicles (SVs). A chemical synapse is composed by three elements: the pre-synaptic terminal, or synaptic bouton, the synaptic cleft and the post-synaptic membrane (Figure 2.9). A 3D model of an average synapse is proposed in [99], where a combination of immunoblotting, mass spectrometry, super-resolution microscopy and EM microscopy is used to quantify and localize synaptic proteins. The model was created from purified synaptic boutons taken from the cellular layer or the cortex and the cerebellum of adult rats. It is like a snapshot of the average synapse in the brain, however it lacks dynamic information. The synapse is a rather crowded environment, as shown in Figure 2.10, limiting, probably, diffusion and dynamics inside the synapse.

The question that we try to address here is: how can we study single proteins interaction in the synapse if it is such a crowded environment? The idea is to 


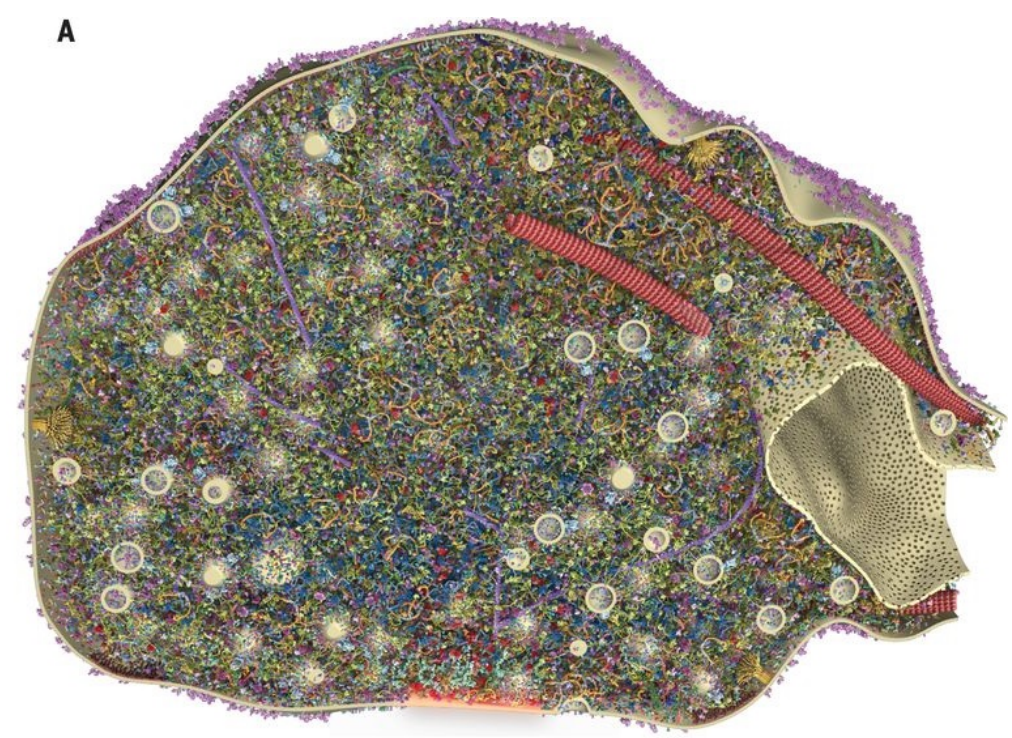

Figure 2.10: 3D model for an average synapse. Cross section on the average synaptic bouton from [99. 60 proteins are shown with copy number in agreement with the measurements and in the location determined by the imaging data. The figure is reprinted from reference [99] under the Creative Commons Attribution (CC BY) license.

create a more simple in vitro primitive version of the synapse and then add the protein to study and measure the interactions using FFS and eventually microfluidics to gain also temporal information. In Section 4.2 data of FCS measurements done in cellulo and in vitro will be presented. Moreover, a microfluidic device that allows to quickly switch the sample measured on the SVs, has been developed.

\section{Synaptic vesicles}

SVs are the storage-transporters for neurotransmitters inside synapses. They are small uniform vesicles with $\approx 20 \mathrm{~nm}$ of radius with at least 40 different [108] proteins at their surface, including trafficking proteins, such as the SNARE proteins or SNAP-25, and transport proteins involved in neutransmitters release and uptake. They are concentrated in the presynaptic terminal of every neurons [108]; in resting neurons, typically, a small part of SVs are docked on the presynaptic membrane while most of them form a "pool" of vesicles behind the first ones [109. They are filled with neurotransmitters thanks to active transport via an electrochemical proton gradient maintained by the V-ATPase. The creation and the maintaining of the proton gradient is called vesicles acidification [154]. The filled SVs docked at the active zone are released from the presynaptic terminal via $\mathrm{Ca}^{2+}$ dependent exocytosis upon the arrival of an action potential. After exocytosis, SVs are recycled and refilled with neurotransmitters, ready to undergo to a new cycle [110] of exocytosis. There are three recycling pathways: they can be 
2.3. Biological background

refilled directly on the presynaptic membrane without undocking from it (called, "kiss-and-stay"), they can undock and be locally refilled (called, "kiss-and-run") or lastly, they can undergo clathrin-mediated endocytosis and recycling through endosomes.

\section{Alpha-synuclein}

Synucleins are a family of three small (127 to 140 aa) soluble proteins found mainly in neural tissue and in some types of tumor [97]. In particular, $\alpha$-synuclein and $\beta$-synuclein are mainly located in the presynaptic terminals in the brain while $\gamma$-synuclein is found mostly in the peripheral nervous system and in breast tumors. Despite the synucleins being so widely spread in the neural tissue, the physiological functions of these proteins are poorly understood [1]. In particular, $\alpha$-synuclein has attracted the attention of neuroscientists because it was identified as one main component of Lewy bodies, the pathological characteristic of different neuronal diseases such as Parkinson. The pathological aggregation of the protein inside the Lewy body might be responsible for some neurodegenerative diseases [4]. The mechanism, by which $\alpha$-synuclein starts to aggregate, is also still unknown. However, it is known that it changes structural conformation if it is bound to lipids. From an intrinsically disordered structure in solution, it forms an extended helical structure on small unilamellar vesicles [101] or lipid membranes [5]. Two apparently diverging theories on the physiological function of $\alpha$-synuclein have emerged during the years. In the first one, $\alpha$-synuclein is supposed to bind to VAMP2 and helps the SNARE complexes during synaptic activity without any effect on neurotranmission [102]. In the other one, $\alpha$-synuclein is considered an attenuator of neurotransmitter release, based on the observation that overexpressing $\alpha$-synuclein attenuates SVs recycling and exocytosis [103]. More recently, a connection between these two theories has been proposed, with a unified model for $\alpha$-synuclein function [104], where through the binding to VAMP2, $\alpha$-synuclein helps to maintain the proper recycling and clustering of SVs. It was shown that $\alpha$-synuclein interacts also with proteins, for example it acts as molecular chaperon assisting folding and refolding synaptic proteins [105], and in general it has been shown to interact with at least 50 proteins [106].

$\alpha$-synuclein also interacts with several polyvalent metal cations including $\mathrm{Fe}^{2+}$, $\mathrm{Cu}^{2+}$ and $\mathrm{Ca}^{2+}[5]$. One factor that probably helps $\alpha$-synuclein to be able to interact so diversely is the ability to modulate the conformation depending on the environment condition. Listed here, are some of the known conformations [5]:

- The intrinsic unfolded state of $\alpha$-synuclein in vitro and in vivo.

- The globular state, that is the predominant state of $\alpha$-synuclein at low $\mathrm{pH}$, high temperature, at the presence of metal ions, with some common pesticides, with polycations and with various salts. 
a.

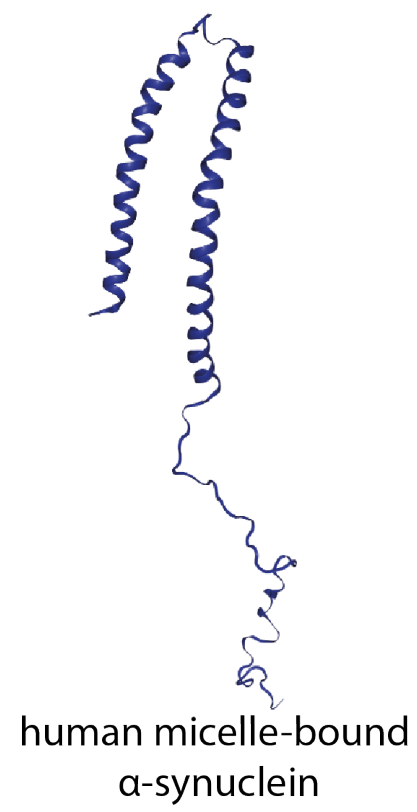

b.

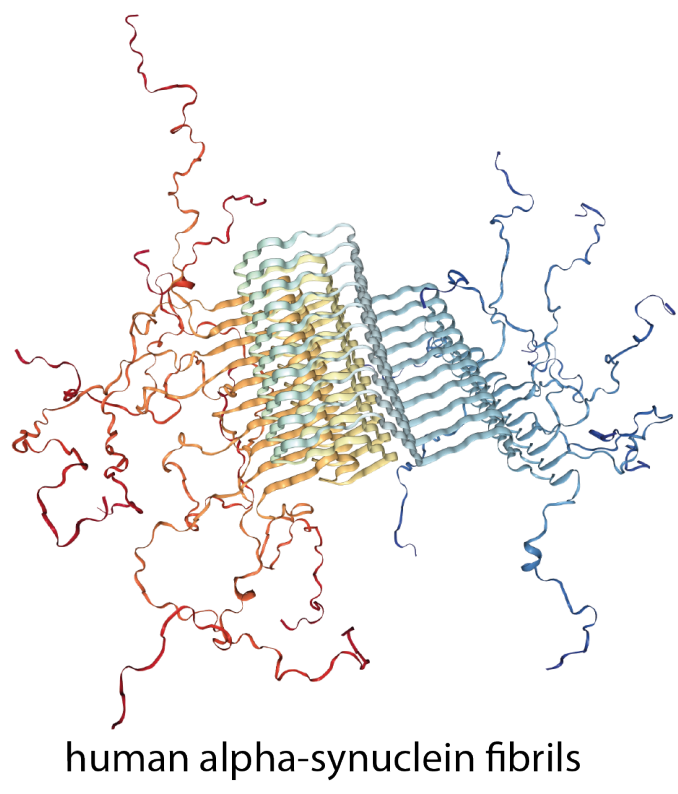

Figure 2.11: Two possible conformations of human $\alpha$-synuclein. a) $\alpha$-synuclein conformation when bound to a micelle. Image from the RCSB PDB (rcsb.org) of PDB ID: 1XQ8 ([54]). b) $\alpha$-synuclein fibril measured by NMR spectroscopy. Image from the RCSB PDB (rcsb.org) of PDB ID: 2N0A ([55]).

- The $\alpha$-helical state, typical when $\alpha$-synuclein is bound to lipid membranes, as shown in Figure 2.11 a. It is shown with circular dichroism that upon addition of small unilamellar vesicles the dichroism spectrum shifts from random coil pattern to an $\alpha$-helical pattern [107.

- A dimeric structure that appears when $\alpha$-synuclein starts to form oligomers and aggregates. It could also be the result of high temperature exposure.

- An oligomeric structure. Starting from the dimers $\alpha$-synuclein can form morphologically different soluble oligomers depending on the type of ions in solution. It can be for example ring like or spherical aggregates.

- Insoluble aggregates. $\alpha$-synuclein can forms two insoluble aggregates, an amorphous aggregate and fibrils. Again the type of aggregate is dependent on the condition of the sample, in most of the cases the major insoluble species are the amyloid-like fibrils (Figure 2.11). These insoluble aggregates might represent the main cause of some neurodegenerative diseases due to their possible toxicity, since they are found in Lewys bodies [100] .

Due to this structural conformation behaviour, $\alpha$-synuclein can be considered as a chameleon-protein that is able to change its structure depending on its surroundings. In vivo, $\alpha$-synuclein exists in equilibrium between the soluble intrinsic 
unfolded state and the helical membrane bound state [111]. Despite more than 25 years of research on $\alpha$-synuclein, the physiological function of this protein is still unclear, nevertheless its importance in neurodegenerative diseases is clear. 


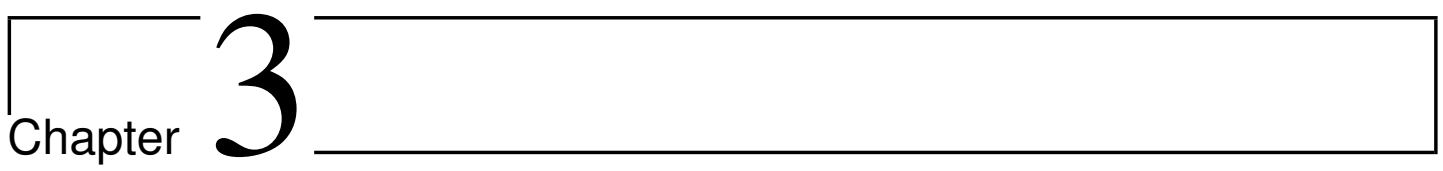

\section{Materials and methods}

In this chapter, first there will be an introduction to the preparation of the biological samples studied in this thesis. Second, the manufacturing of the microfluidic devices employed and the sample preparation will be described in detail. At last, the experimental settings and the methods used to analyze the data, will be reported.

\subsection{Protein preparation}

\subsubsection{Vimentin protocols}

Human vimentin C328A, carrying three additional amino acids GGC at the Cterminus, is recombinantly expressed in Escherichia coli bacteria with a protocol adapted from [113. Expression and purification of vimentin is performed in-house by Susanne Bauch. The plasmid used for bacteria transfection is provided by Harald Herrmann, DKFZ Heidelberg, Germany. This type of mutation is used to label vimentin via a maleimide reaction at the mutated cysteine. Since attaching the label at the rod domain cysteine (aa 328) of wild type vimentin affects the assembly process, the cysteine at 328 is replaced with alanine. Vimentin is labeled with Atto-532-maleimide (AttoTech GmbH, Siegen, Germany) as described in [114. Briefly, vimentin is dialyzed, using dialysis tubing with a cut of at $50 \mathrm{kDa}$ (Spectra/Por7, dialysis membrane made of regenerated cellulose, MWCO50000, Carl-Roth GmbH, Karlsruhe, Germany), into labeling buffer (5M urea, 50mM phosphate buffer pH $7.5\left(\mathrm{~PB}, 50 \mathrm{mM} \mathrm{NaH} \mathrm{PO}_{4}, 50 \mathrm{mM} \mathrm{Na}_{2} \mathrm{HPO}_{4}\right)$, all chemicals are from Carl Roth $\mathrm{GmbH}$ ). The dye is dissolved in water-free DMSO (Dimethyl sulphoxide, Carl Roth GmbH) to a concentration of $10 \mathrm{mM}$. $20 \mu \mathrm{L}$ of dye solution are added in steps of $5 \mu \mathrm{L}$ to the vimentin (concentration $1 \mathrm{~g} / \mathrm{L}$ ). After every addition of the dye, the solution is vortexed and incubated for 5 minutes. Once all the $20 \mu \mathrm{L}$ of dye are added to the vimentin, the solution is incubated for two hours. After the incubation, $100 \mu \mathrm{L}$ of $1 \mathrm{M}$ cysteine (Carl Roth $\mathrm{GmbH}$ ) are added 


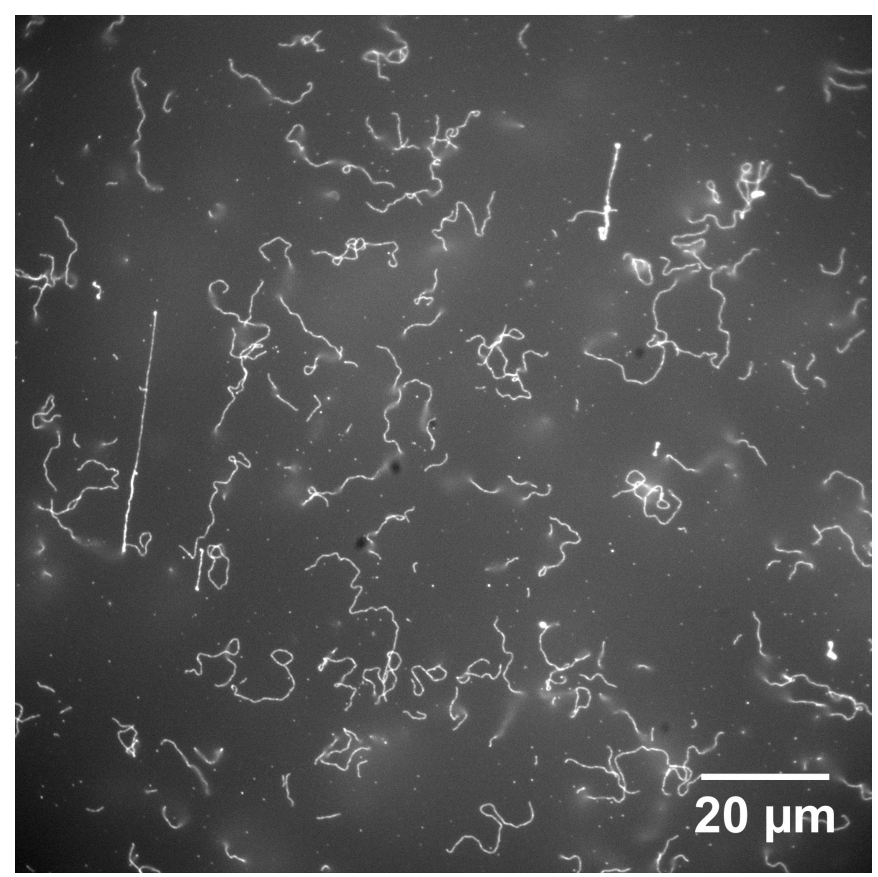

Figure 3.1: Fluorescence image of vimentin filaments after $8 \mathrm{~h}$ of in vitro assembly at $37^{\circ} \mathrm{C}$ with a protein concentration of $0.1 \mathrm{~g} / \mathrm{L}$. Labeled and unlabeled vimentin monomers are mixed at a ratio of $25 \%$ labeled protein.

to the mixture of vimentin-dye for 1 hour. This step is fundamental to remove excess free dye in the solution that might bind unspecifically to vimentin. Free dye and labeled vimentin are separated using a $300 \mathrm{~mm}$ Bio-Gel P-30 (Bio-Rad Laboratories GmbH, Feldkirchen, Germany) polyacrylamide gel column via size exclusion chromatography. The column, with a bed volume of $24 \mathrm{~mL}$, is prepared as recommended in the instruction manual. The vimentin-dye solution is loaded on top of the column and it is allowed to enter completely in the gel bed. To flush the column the labeling buffer is used. Fractions of labeled protein are collected in aliquots of about $250 \mu \mathrm{L}$. Vimentin and dye concentrations are measured by UVvis absorption spectroscopy (Nanodrop ND-1000, Thermo Scientific Technologies, Inc., Wilmington, USA) and peak fractions are pooled. The labeled protein is dialyzed against storage labeling buffer $(8 \mathrm{M}$ urea in $2 \mathrm{mM} \mathrm{PB}, \mathrm{pH}=7.5)$ and the protein is stored at $-80^{\circ} \mathrm{C}$. Before the experiment, a mixture of labeled and unlabeled vimentin (between $25 \%$ to $30 \%$ labeled protein) is dialyzed in a step-wise manner $(6 \mathrm{M}, 4 \mathrm{M}, 2 \mathrm{M}, 1 \mathrm{M}, 0 \mathrm{M})$, into $2 \mathrm{mM} \mathrm{PB}(\mathrm{pH}=7.5)$ at room temperature, using a $50 \mathrm{kDa}$ cut-off membrane. Afterwards, an additional step is performed at $8^{\circ} \mathrm{C}$ into $2 \mathrm{mM} \mathrm{PB}$ overnight. Finally, the protein is further dialyzed at room temperature for 1 hour into fresh $\mathrm{PB}$. At $2 \mathrm{mM}$ of $\mathrm{PB} \mathrm{pH} 7.5$ vimentin molecules have already assembled into tetramers. The protein concentration and labeling ratio are determined again from absorption data at $280 \mathrm{~nm}$ (Nanodrop 
ND-1000). In vitro, the assembly is started by mixing equal volumes of assembly buffer (2mM PB with $200 \mathrm{mM} \mathrm{KCl,} \mathrm{pH} \mathrm{7.5)} \mathrm{and} \mathrm{vimentin} \mathrm{(concentration} \mathrm{of}$ $0.2 \mathrm{~g} / \mathrm{L}$ ), diluting the salt concentration to $100 \mathrm{mM} \mathrm{KCl}$. A typical image of vimentin filaments is presented in Figure 3.1 after 8 hours of assembly at $37^{\circ} \mathrm{C}$ at a concentration of $0.1 \mathrm{~g} / \mathrm{L}$ with a labeling ratio of $25 \%$.

\subsection{Printing synaptic vesicles on glass surfaces}

SVs are attached to the glass coverslips via a neutravidin pattern. The antibodies and the SVs employed for the patterning are provided by the group of Prof. Rizzoli of the University Medical Center of Göttingen in the Institute for Neuroand Sensory Physiology and Biostructural Imaging of Neurodegeneration, who is our collaborator for this project. To create a pattern of SV on a microscope glass coverslip (24x60 mm, No.1 thickness, VWR, Radnor, Pennsylvania, USA), the PRIMO micropattening system (Alveole, Paris, France) is employed. The PRIMO system is mounted on top of an Olympus IX 83 microscope (Olympus IX73, Olympus Europa SE \& CO. KG, Hamburg, Germany). Glass coverslips are cleaned by rinsing them with isopropanol. After drying the coverslips with $\mathrm{N}_{2}$, air plasma treatment (ZEPTO, plasma cleaner, Diener electronics GmbH, KG Ebhausen, Germany) is applied on them for 3 minutes at $40 \mathrm{~W}$.

Subsequently to the plasma treatment, a PDMS stencil, that creates a circular chamber which will contain the patter (diameter $3.5 \mathrm{~mm}$ ), is applied to the surface and $20 \mu \mathrm{L}$ of $0.1 \mathrm{mg} / \mathrm{mL}$ of PLL-g-PEG (PLL(20)-g[3.5]-PEG(2 kDa), SuSoS AG, Dübendorf, Switzerland) diluted in phosphate buffered saline (PBS 10X stock solution: $1.37 \mathrm{M} \mathrm{NaCl}, 27 \mathrm{mM} \mathrm{KCl}, 43 \mathrm{mM} \mathrm{Na} \mathrm{HPO}_{4} \cdot 12 \mathrm{H}_{2} \mathrm{O}, 14 \mathrm{mM} \mathrm{KH} \mathrm{PO}_{4}$; working solution $1 \mathrm{X}$ ) is added into the PDMS well and incubated for 1 hour. The PLL-g-PEG coating provides the coverslip with anti-fouling properties preventing unspecific protein adsorption (Figure $3.2 \mathrm{a}$ ). After rinsing three times with PBS, 8$10 \mu \mathrm{L}$ of UV-sensitive photoinitiator (PLPP, Alveole) are added into the PDMS well (Figure $3.2 \mathrm{~b}$ ). To create the virtual mask with the pattern (in this case circular dots with $130 \mu \mathrm{m}$ diameter), the open source software Inkscape is used. The pattern is loaded into the PRIMO software, Leonardo, and a $20 \mathrm{X}$ objective (Olympus LUCPLFLN 20X, NA=0.45) projects UV light through the virtual designed mask (Figure 3.2c). The PLPP, once activated by UV light, degrades the anti-fouling layer of PLL-g-PEG, leaving the exposed regions available for the attachment of the protein of choice (Figure 3.2 d). A dose between 1800 and 2000 $\mathrm{mJ} / \mathrm{mm}^{2}$ is sufficient to have the protein uniformly coating the pattern. After patterning, the PLPP is removed by washing three times with PBS and then neutravidin (Thermo Fisher Scientific, Waltham, MA USA) at a concentration of $0.05 \mathrm{mg} / \mathrm{mL}$ is added to the pattern in the PDMS well. The protein is incubated for at least two hours, and after washing of the remaining protein with PBS, the pattern is ready to be used.

Anti-synaptotagmin-biotinlated mouse monoclonal antibodies (synaptotagmin1 
PLL-g-PEG anti-fouling coating

a.

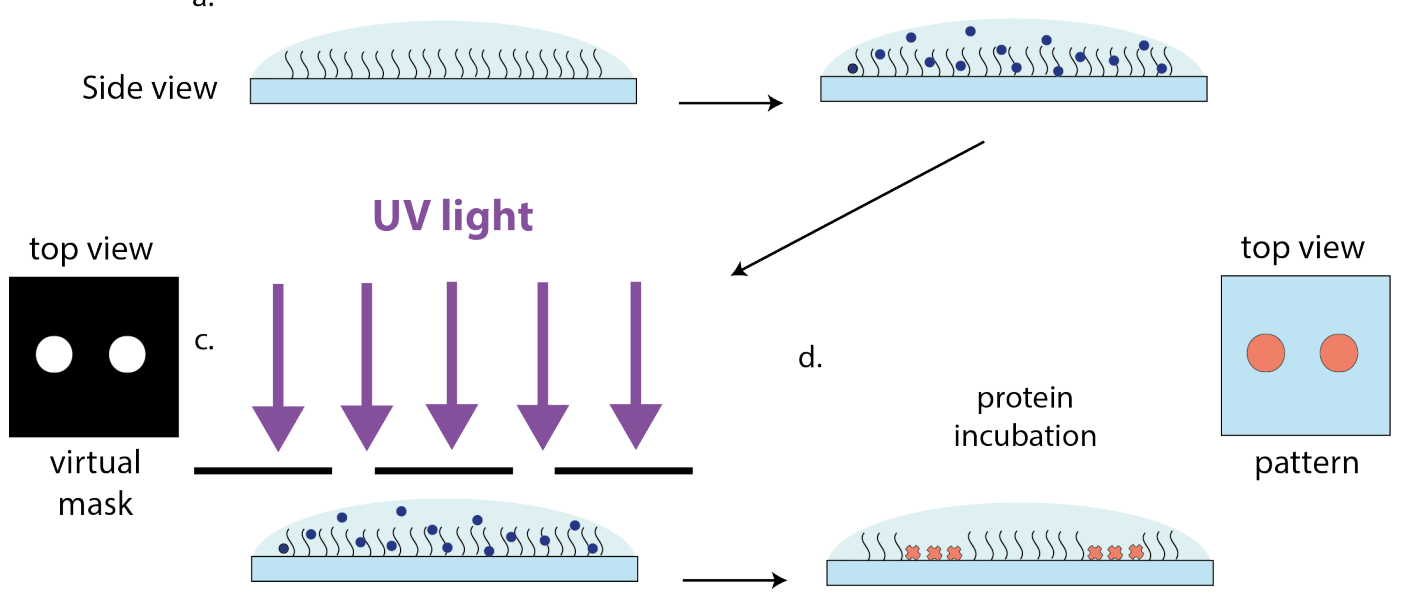

Figure 3.2: Patterning of a glass coverslip using PRIMO micropatterning system. (a) The substrate, in this case a glass coverslip, is uniformly coated with an anti-fouling layer of PLLg-PEG. (b) The photoinitiator (PLPP) is added on top of the PLL-g-PEG layer and (c) the substrate is exposed with UV light through a photomask. (d) Under UV light, the PLPP is activated and it degrades the anti-fouling layer of PLL-g-PEG, leaving available regions for the protein of choice to attach.

cytoplasmic tail, mouse monoclonal, purified IgG, biotin-labeled, Synaptic Systems GmbH, Göttingen, Germany), diluted 1 to 100, are added to the pattern and incubated for 1 hour. During the incubation time, SVs (purified by the group of Prof. Rizzoli) are incubated with FluoTag anti-VGLUT1-STAR635, a single domain antibody with one antigen binding site for the glutamate-1 transporter in the membrane of synaptic vesicles (NanoTag Biotechnologies GmbH, Göttingen, Germany). After one hour, the pattern is washed with PBS 3 times and the labeled SVs are incubated with the pattern for 1 hour. Subsequently the pattern is washed three times with PBS to remove free SVs.

\subsection{Microfluidic devices}

In this thesis, different types of microfluidic devices are employed. In particular, for the study of the protein assembly two designs of devices are utilized: a five-inlet device to study protein aggregation (device B) and a single channel device to characterize the protein and the method (device A). To measure protein interactions with printed SVs, a three-inlet device is employed. 


\subsubsection{Microfluidic device to study protein aggregation}

All the master wafers are fabricated in the class 1000 clean room of the physics faculty at the University of Göttingen by standard photo-lithography methods [115, 116]. The device for testing the PCH behavior at different velocities (device A) is a simple straight channel (channel length $2.5 \mathrm{~cm}$, width $250 \mu \mathrm{m}$, height $25 \mu \mathrm{m}$ ). Briefly, SU8-3025 (MicroChem., Newton, MA, USA) is spin coated on a 2-inch silicon wafer (MicroChemicals GmbH, Ulm, Germany) to a final height of $25 \mu \mathrm{m}$. The velocities used to achieve a certain photo-resist thickness on the wafers are summarized in Table 3.1. The wafers are soft baked for 15 minutes at $95^{\circ} \mathrm{C}$, exposed to UV light (MJB4 Mask-Aligner, Süss MicroTec AG, Garching, Germany) through a photo mask (Selba S. A., Versoix, Switzerland), post baked $\left(5\right.$ minutes at $95^{\circ} \mathrm{C}$ ), and developed (MR-Dev-600, Micro Resist Technologies GmbH, Berlin, Germany).

The device for studying protein assembly (device B) is a five-inlet and oneoutlet device with a channel width of $200 \mu \mathrm{m}$ and a height of $100 \mu \mathrm{m}$ for the central inlet, and $200 \mu \mathrm{m}$ for the remaining channels. The height constriction in the central channel helps to engulf the protein central stream into a buffer layer, preventing the protein to touch the channel walls. Two different wafers are produced by photo-lithography for device B, (i) the five-inlet geometry (master 1) and (ii) a "step" for the protein inlet $(200 \mu \mathrm{m}$ width and $50 \mu \mathrm{m}$ thickness, master 2) to create one side of the height constriction, as sketch in Figure 3.3. To create the second half of the constriction on master 1, a 3D structure is created using two layers $(150 \mu \mathrm{m}$ and $50 \mu \mathrm{m}$ thick) of SU8-3050 photo-resist. To improve the uniformity of the first layer, the resist is spin coated twice with a height of $75 \mu \mathrm{m}$ each (Figure 3.3 a). After the first layer, the wafers are soft baked for 2 hours at $95{ }^{\circ} \mathrm{C}$. The first layer is exposed to UV-light through a photomask (Figure $3.3 \mathrm{~b}$ ). After the post exposure bake, 20 minutes at $95{ }^{\circ} \mathrm{C}$, the wafers are spin coated with the second layer of photo-resist with a thickness of $50 \mu \mathrm{m}$ (Figure 3.3.). The wafers are soft baked again at $95^{\circ} \mathrm{C}$ for 30 minutes, aligned to the first structure and exposed to UV light once more, using a second mask (Figure $3.3 \mathrm{~d}$ ). The wafers are baked for 15 minutes at $95^{\circ} \mathrm{C}$ and the photo-resist is developed (Figure 3.3p). Master 2 is fabricated by spin coating SU8-3050 to a height of $50 \mathrm{\mu m}$ (Figure $3.3 \mathrm{~F}$ ). After being soft baked at $95^{\circ} \mathrm{C}$ for 30 minutes, the wafers are exposed to UV light (Figure 3.3p). Afterwards, the wafers are baked again for 15 minutes at $95^{\circ} \mathrm{C}$ and then developed (Figure $3.3 \mathrm{~h}$ ). All masters are coated with (heptafluropropyl)-trimethylsilane (Aldrich, Steinheim, Germany) overnight. The coating helps to remove the PDMS replicas from the master wafers.

Polydimethylsiloxane (PDMS, Sylgard 184, Dow Corning, Midland, MI, USA) replicas are fabricated from the master structures (ratio polymer:cross-linker = 10:1, at $65{ }^{\circ} \mathrm{C}$ for 1 hour) and holes are punched at the inlets channels (biopsy puncher, $0.75 \mathrm{~mm}$ diameter, World Precision Instruments, Sarasota, FL, USA) for connecting the tubing to the device. The top part of the central channel 


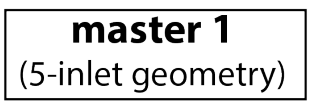

a.

first layer of photo-resist

\begin{tabular}{|c|}
\hline SU8-3050 \\
\hline Si wafer
\end{tabular}

first exposure

b.

UV light

mask 1

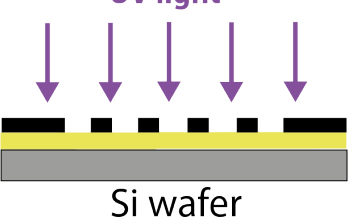

c.

second layer of photo-resist

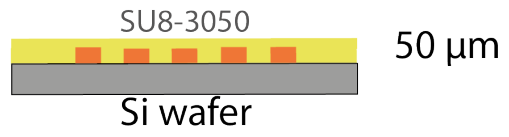

mask alignment

$\&$

d. second exposure

uv light

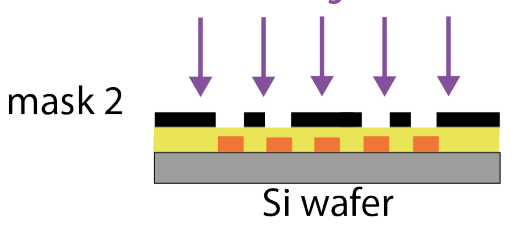

e. photo-resist

development

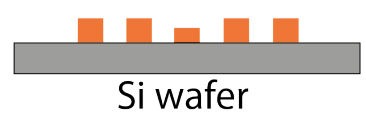

f.

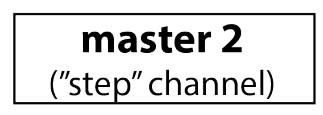

layer of photo-resist

sU8-3050

\begin{tabular}{|c|}
\hline Si wafer \\
\hline
\end{tabular}

exposure

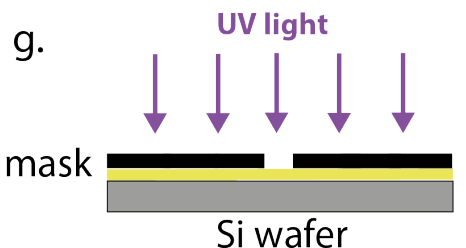

Si wafer

h.

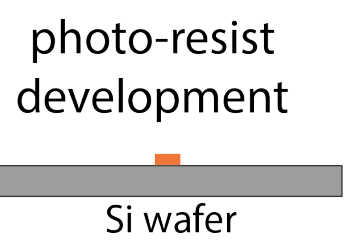

Si wafer 


\begin{tabular}{ccccc}
\hline $\begin{array}{c}\text { Height } \\
{[\mathrm{\mu m}]}\end{array}$ & Resist & $\begin{array}{c}\text { Duration } \\
{[\mathrm{s}]}\end{array}$ & $\begin{array}{c}\text { Speed } \\
{[\mathrm{rpm}]}\end{array}$ & $\begin{array}{c}\text { Ramp } \\
{[\mathrm{rpm} / \mathrm{s}]}\end{array}$ \\
\hline \hline 25 & SU8-3025 & 30 & 500 & 100 \\
& & 30 & 1000 & 200 \\
& & 40 & 4000 & 300 \\
\hline 50 & SU8-3050 & 5 & 500 & 100 \\
& & 30 & 2000 & 200 \\
\hline 75 & SU8-3050 & 5 & 500 & 100 \\
& & 30 & 1000 & 200 \\
& & 40 & 1850 & 300 \\
\hline 100 & SU8-3050 & 5 & 500 & 100 \\
& & 30 & 800 & 200 \\
& & 40 & 1000 & 300 \\
\hline
\end{tabular}

Table 3.1: Spin coating velocities used to achieve the corresponding resist heights.

constriction for device $b$ is integrated in the PDMS replica stemming from master 1. For the bottom part, a step is created directly on the glass coverslip (number 1, ThermoScientific Technologies, Inc., Wilmington, USA) using master 2 (Figure 3.4). A cut-open PDMS replica of master 2 is placed on top of a clean glass coverslip (Figure 3.4 a) and a drop of liquid adhesive (NOA H83, Norland Optical Adhesives, Cranbury, NJ, USA) is used to create a step, exploiting the technique of micromolding in capillaries (MIMIC) [117]. The liquid adhesive fills the channel by capillary forces (Figure $3.4 \mathrm{~b}$ ). To speed up the process, the glass slide with the liquid adhesive is placed in a desiccator. The liquid adhesive is then cured by UV light (Figure 3.4c) at $365 \mathrm{~nm}$ for 3 hours. Afterwards, the PDMS channel is removed $(3.4 \mathrm{~d})$ and the glue is further cured under UV light for 30 minutes. To covalently bind the two sides of the microfluidic device air plasma activation is used. Both, the PDMS channel replica and the glass slide with the glue step are cleaned with isopropanol, dried with nitrogen and exposed to air plasma (ZEPTO, plasma cleaner, Diener electronics $\mathrm{GmbH}$ ) for 12 seconds at $40 \mathrm{~W}$. They are aligned under a stereo microscope (Olympus SZ61) and pressed together to form a covalent bond (Figure 3.4 e). The device is placed on an heating plate at $95^{\circ} \mathrm{C}$ for 2 hours to improve the strength of the bond.

\subsubsection{Microfluidic device to study patterned SVs under a switchable flow}

This project is in collaboration with the group of Prof. Reinhard Jahn from the Laboratory of Neurobiology of the Max Plank Institute for Biophysical Chemistry in Göttingen. To measure the interaction of molecules with printed SVs in flow, a three-inlet device (channel length $2.3 \mathrm{~cm}$, width $1 \mathrm{~mm}$ and height $100 \mu \mathrm{m}$ ) is 


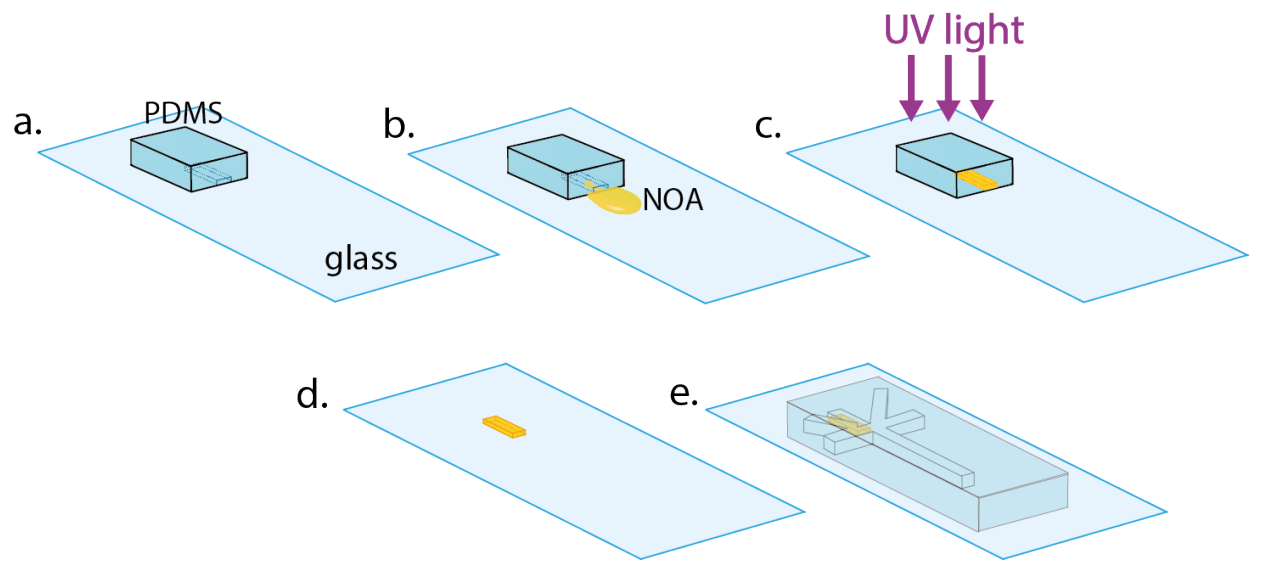

Figure 3.4: Sketch of the construction of the five-inlet microfluidic device to study protein aggregation. The central channel of the microfluidic device has a constriction to reduce the height. One side of the constriction is already integrated in the PDMS replica of master 1 . The second side of the constriction is created directly on the glass coverslip. a) A cut-open PDMS replica of master 2 is applied to a clean glass slide. b) A drop of liquid adhesive (NOA H83) fills, via capillary forces, the channel. c) After being cured by UV light for three hours, the liquid adhesive channel is furthermore cured under UV light without the PDMS slab. d) The cured glue channel is aligned with the PDMS replica of master 1 and the two sides are bound after air plasma treatment. e) The device is ready to be employed in experiments.

employed. This microfluidic device is used to flush different fluids on top of the $\mathrm{SV}$ pattern in a controlled manner. To measure how fast, for example, the SVs react to a different $\mathrm{pH}$ or to a particular molecule, we have to be able to change the reactant agent quickly. The idea is to have a device with the SV pattern in the center of the outlet channel and to control the flow in a way to be able to switch the solution flowing, for example, from the one contained in inlet 1 to the one in inlet 2, in a controlled and fast manner, as shown in Figure 3.5. To achieve the switching of the solution flowing on top of the patterned SV, a rectangular function is applied to the side inlets of the microfluidic device, with a maximum velocity of $1 \mathrm{~mm} / \mathrm{s}$, a minimum velocity of $0.1 \mathrm{~mm} / \mathrm{s}$ and width $10 \mathrm{~s}$, as shown in Figure 4.38a (Section 4.2.3). When the flow from the side-1 channel has the maximum velocity, as in Figure 3.5 , the flow from the side- 2 channel has the minimum velocity. In the central outlet, the flow from side- 1 pushes the solution from side-2 towards the channel wall, filling the central part of the outlet channel where the SV are patterned. When the flow from the side-1 channel has the minimum velocity, as in Figure $3.5 \mathrm{~b}$, the flow from the side-2 channel has the maximum velocity. In this case, the solution from side- 1 channel is pushed away from the central part of the outlet by the solution from side- 2 channel. Thus, the patterned SVs are now exposed to the solution coming from the side-2 inlet.

The master wafers are created in the 1000 class clean room of the physics faculty at the University of Göttingen by standard photo-lithography methods [15, 116]. Briefly, a $100 \mu \mathrm{m}$ layer of negative photo-resist (SU8-3050) is uni- 

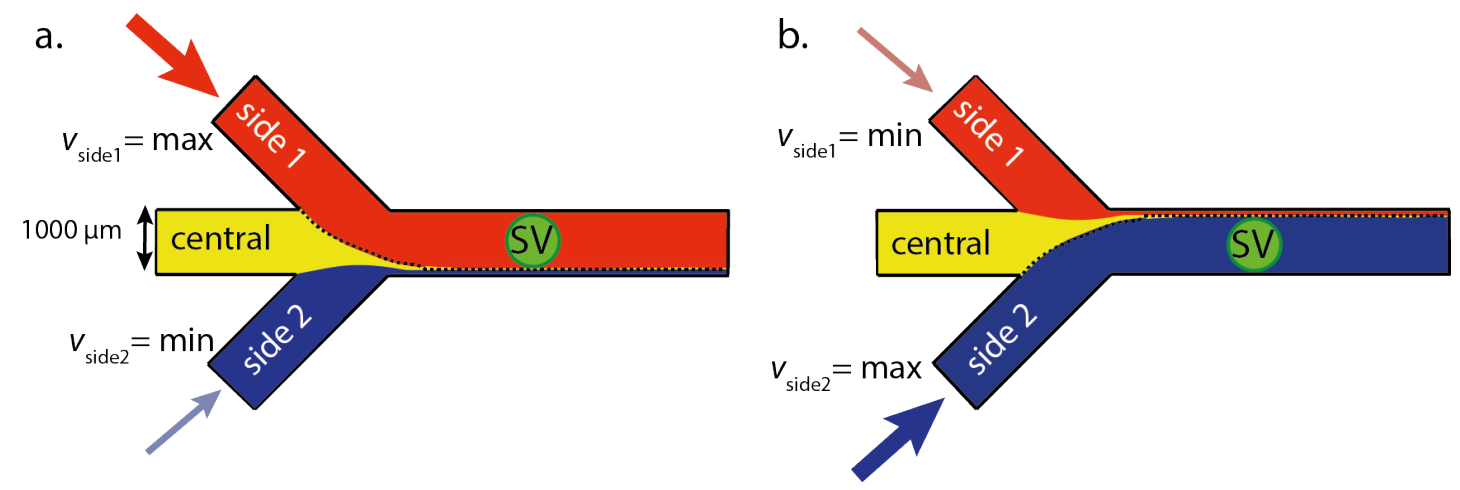

Figure 3.5: Schematic representation of the microfluidic device to study SVs under a switchable flow. When the velocity is maximum in one side inlet, for example side 1 , the solution from that specific inlet fills the central outlet channel. When the velocity from that side inlet reaches the minimum, the solution from that inlet is not anymore in the center of the device, but the solution from the second side inlet fills the outlet channel.

formly spin coated on top of a 2-inch cleaned silicon wafer with velocities according to Table 3.1. The wafers are soft baked at $95^{\circ} \mathrm{C}$ for 45 minutes and then exposed to UV light through a photomask via the mask aligner. The wafers are baked again for 15 minutes at $95^{\circ} \mathrm{C}$ and developed for 15 minutes. After cleaning the wafers with isopropanol, they are coated overnight with (heptafluropropyl)trimethylsilane. PDMS replicas are created by mixing the liquid PDMS with the cross-linker at a ratio of 10:1 and pouring the mixture on the wafers. After removing the air-bubbles with a desiccator, the PDMS is cured in the oven (DryLine, VWR, Darmstadt, Germany) at $65{ }^{\circ} \mathrm{C}$ for 1 hour.

As in this case, SVs are patterned on the glass surface, which is the bottom of the microfluidic device, plasma treatment cannot be used to covalently bind the PDMS channel replica with the glass coverslip because the plasma would destroy the previously patterned SVs on the surface. To assemble the device, the PDMS channel and the patterned glass coverslips are placed inside an home-made device holder (a sketch is presented in Figure 3.6) inspired by [118, 134]. The device holder consists of two metal plates clamped together with 8 bolt screws. The patterned glass coverslip is inserted between the two plates. A window in the bottom metal plate allows us to image the patterns with a microscope. The PDMS replica with the channel is aligned on top of the glass coverslip effectively closing the device. Uniform pressure on the device is achieve by inserting a plastic (PVC), or glass, plate (1 $\mathrm{mm}$ of thickness) before the second metal plate. The uniform pressure prevents also leaking of the device. Tubings are inserted to the device through holes in the top metal plate and in the plastic plate. A photograph of the microfluidic device mounted in the holder is shown in Figure 3.6 b. 
a.

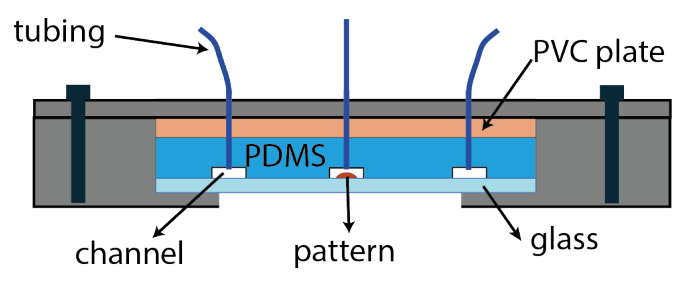

b.

Top view
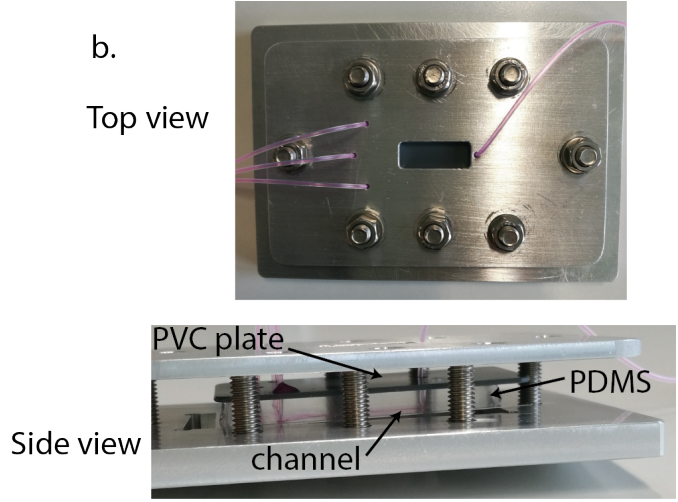

Figure 3.6: Device holder for microfluidic chips with patterned molecules on the glass coverslip. The microfluidic device is held together by two metal plates screwed together via 8 bolts screws. The bottom metal plate has an opening to allow us to image the microfluidic device in an inverted microscope. The PDMS replica of the channels is mounted on top of the patterned glass slide closing the microfluidic device. On top of the PDMS, a thick glass slide or a PVC plate is used to uniformly spread the pressure on the device preventing leakage. Tubings are inserted via holes on the top metal plate and the PVC plate.

\subsection{Setup for FFS and experimental conditions}

In this section, the experimental conditions for the experiments reported in the following sections are reported. In particular, the confocal microscope and the epi-fluorescence microscope, employed in the FFS experiments, will be described. Following the setup descriptions, the data acquisition and analysis will be illustrated.

\subsubsection{Confocal microscopy}

The setup used is based on a modified inverted microscope (Olympus IX73, Olympus Europa SE \& CO. KG, Hamburg, Germany). The excitation light is provided by two diode pulsed lasers (Cobolt Samba-532 100mW and Cobolt Calypso-491 25mW, Cobolt AB, Solna, Sweden) inserted into a laser combiner box (C-Flex, Cobolt AB, Solna, Sweden). After exiting the optical fiber, the laser light passes through a clean-up filter (HC Laser Clean-Up MaxLine 491/1.9, AHF Analysentechnik, Tübingen, Germany HC Laser Clean-up MaxLine 532/2). The laser beam is expanded by a factor of 10 using a 10X objective (Olympus UPLFLn10XP, $\mathrm{NA}=0.30$ ) and a $\mathrm{f}=200 \mathrm{~mm}$ lens (Qioptiq Photonics $\mathrm{GmbH}$ KG, Göttingen, Germany) in order to illuminate the full back aperture of the microscope objective. The laser intensity is attenuated with a neutral density filter $(\mathrm{OD}=6$, Qioptiq Photonics) before being deflected by a dichroic mirror (DualLine zt488/532rpc, AHF Analysentechnik AG, Tübingen, Germany) into the microscope. The laser beam is focused onto the sample using a $60 \mathrm{X}$ water immersion objective (UPlanApo, $\mathrm{NA}=1.2$, Olympus). The fluorescence light is 


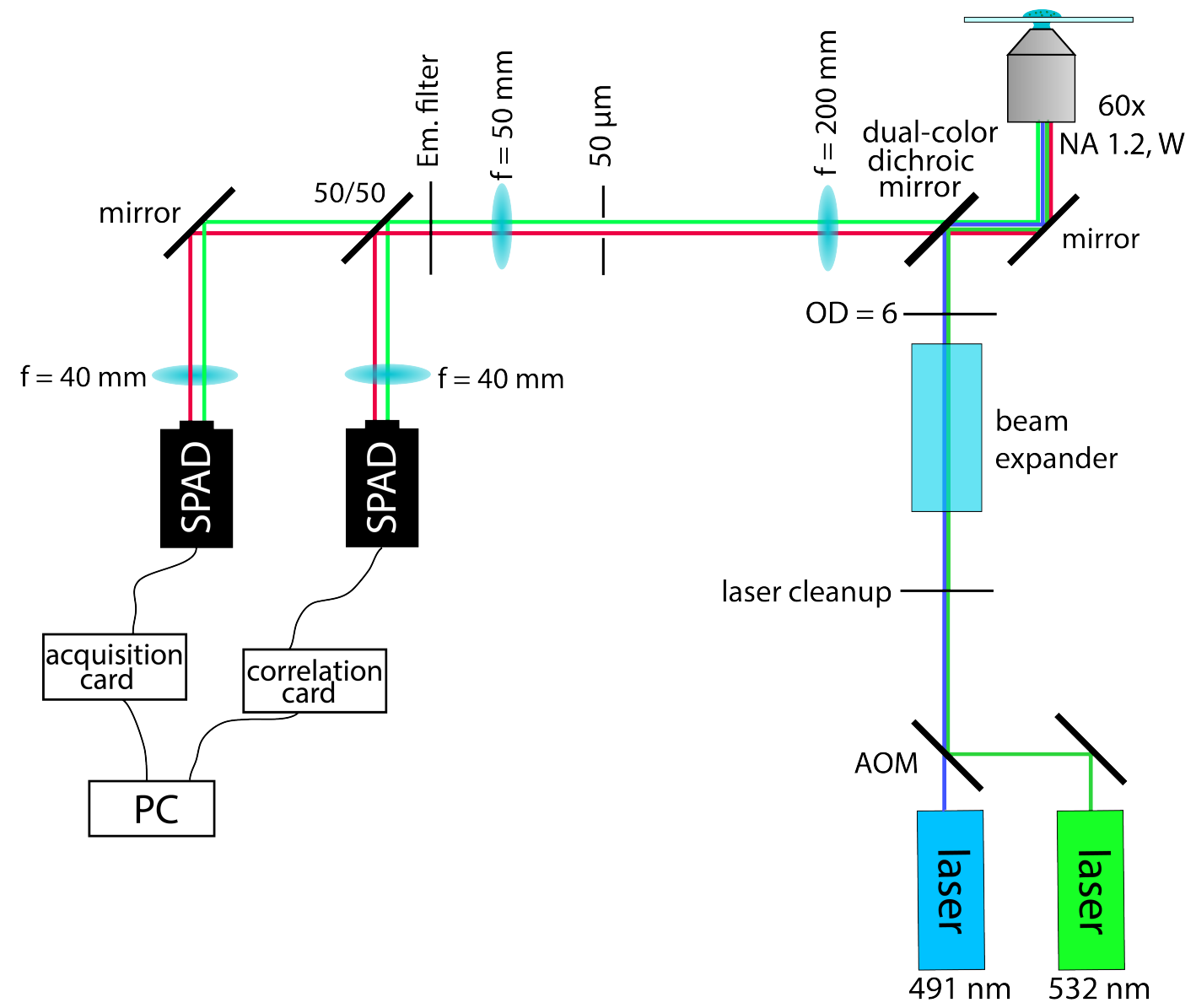

Figure 3.7: Schematic representation of the confocal microscopy setup.

then focused using a $\mathrm{f}=200 \mathrm{~mm}$ lens to the pinhole (diameter $50 \mu \mathrm{m}$, Qioptiq Photonics) cutting off the out off focus light contribution. After the emission filter (razor Edge Long Pass Filter 488 or RazorEdge LP Edge Filter 532, AHF analysentechnik AG) the light is collimated using a $\mathrm{f}=50 \mathrm{~mm}$ lens and directed to the active area of two different avalanche photo diodes $(\tau$-SPAD, Picoquant GmbH, Berlin, Germany and CountBlue Count50, Laser Components GmbH Olching, Germany), using a 50:50 beam splitter (Thorlabs BSW10R, Thorlabs Inc. Newton, New Jersey, USA). A scheme of the optical path is shown in Figure 3.7.

The $\tau$-SPADs are connected either to a digital correlator card (ALV-7004 USB, ALV-Laser Vertriebsgesellschaft mbH, Langen, Germany) used for autocorrelation measurements, or to an acquisition card (NI-6602, National Instruments, Austin, USA) to access directly the raw photon arrival times. The digital correlator can calculate four correlation functions simultaneously: the autocorrelation functions of the two distinct APDs and the forward and backward cross-correlation functions between the two detector signals. In principle, it is also possible to measure 
2-color cross-correlation functions, if the 50:50 beam splitter is replaced by a dichroic mirror. The digital correlator card is directly connected to the PC to store and analyze the data. To access the data from the acquisition card, we use a custom-written code written using on a python interface [123]. The photon arrival times are measured relative to an arbitrary start point with an internal clock of $10 \mathrm{MHz}$. All the experimental data are then analyzed using self-written Python code (Python Software Foundation, https://www.python.org/). To access different positions in the sample, an automated sample stage (Prior Scientific, Inc., Rockland, MA, USA) is used.

\subsubsection{Epi-fluorescence microscopy}

The same microscope described above can also be used for epi-fluorescence microscopy. A mirror in the second deck of the microscope body allows us to switch between the two microscope configurations. The excitation light comes from a mercury arc lamp (X-Cite 120 PC Q, Excelitas Technologies) and it is guided onto a fluorescence filter cube (filter sets available: DAPI, GFP, Cy3, TxRed and Cy5, all from AHF analysentechnik AG, Tübingen) which selects the wavelength of the excitation and filters the emission light. Images are acquired using a CCD-camera (Hamamatsu Orca R-2, Hamamatsu Photonics Deutschland GmbH, Herrsching am Ammersee, Germany) controlled by Micro-Manager [124].

\subsubsection{Data acquisition and analysis}

\section{Settings for microfluidic experiments}

Flow measurements are performed using the microfluidic devices described in Section 3.3. Polyethylene tubings (inner diameter $0.38 \mathrm{~mm}$, outer diameter $1.09 \mathrm{~mm}$, Intramedic Clay Adams Brand, Becton Dickinson and Company, Sparks, USA) are connected to Hamilton Gastight glass syringes (Bonaduz, Switzerland) using disposable needles. For a precise control of the flow, syringe pumps (neMESYS, Cetoni GmbH, Korbußen, Germany) are employed to regulate the flow rates of the syringes connected to all the inlets. In the microfluidic experiments where vimentin assembly is studied, Chapter 4.1, the starting vimentin concentration is $0.003 \mathrm{~g} / \mathrm{L}$ and the assembly is initiated by adding $100 \mathrm{mM} \mathrm{KCl}$ by diffusive mixing. Flow rates in these experiments are $12 \mu \mathrm{L} / \mathrm{h}$ (central inlet), $10 \mu \mathrm{L} / \mathrm{h}$ (sheath inlets) and $190 \mu \mathrm{L} / \mathrm{h}$ (side inlets).

In the experiments, where SVs interactions under flow are investigated, in Chapter 4.2.3, the total inflow velocity used is $3 \mathrm{~mm} / \mathrm{s}$ and it is divided over the three inlets. In the central channel a constant velocity of $0.1 \mathrm{~mm} / \mathrm{s}$ is employed, while in the side inlets a periodic rectangular anti-synchronous flow velocity profile is applied. As shown in Figure 4.38, the velocity profile of the side inlets has a maximum flow velocity of $1 \mathrm{~mm} / \mathrm{s}$, a minimum of $0.1 \mathrm{~mm} / \mathrm{s}$ and a width of $10 \mathrm{~s}$. 
The velocities in the two side inlets are shifted in time to achieve a constant flow velocity in the center of the channel.

\section{FCS experiments}

Stationary FCS or PCH measurements are performed using $300 \mu \mathrm{L}$ of sample placed in an eight-well glass slide (Nunc Lab-Tek chamber slides, Thermo Fisher Scientific, Pittsburg PA). Before every measurement on the confocal microscope, the setup is aligned and the observation volume is measured. The calibration procedure is fundamental to determine the absolute value of the diffusion coefficients [125] obtained during the measurements. The diameter of the observation volume is calculated measuring the autocorrelation function of dyes with a known diffusion coefficient. For measurements at $532 \mathrm{~nm}$, rhodamine 6G (Thermo Fisher, $D=414 \pm 5 \mathrm{~mm}^{2} / \mathrm{s}$ at $\left.25{ }^{\circ} \mathrm{C}[126]\right)$ is used. For measurements at $491 \mathrm{~nm}$, Atto 488 (AttoTech GmbH, Siegen, Germany , $D=400 \pm 10 \mu \mathrm{m}^{2} / \mathrm{s}$ at $25^{\circ} \mathrm{C}$ [127]) is used respectively. The measurements are usually performed at a temperature of $22{ }^{\circ} \mathrm{C}$. The detection volume $\omega_{0}$ is then calculated with:

$$
\omega_{0}=\sqrt{4 D \tau_{D}}
$$

Usually the measured diameter is $300 \pm 10 \mathrm{~nm}$. For FCS measurements, data are collected between 10 and $60 \mathrm{~s}$ for each curve, depending on the expected diffusion coefficient. The data are analyzed either using the software Quickfit 3.0 [128] or using a self-written fitting routine with Python code (Python Software Foundation, https://www.python.org/). Measurements, where strong fluorescence peaks, caused by aggregates, affect the correlation curves are excluded from the analysis. If bleaching occurs, the trace of fluorescence intensities over time is fitted using a double exponential [129] and the data are re-correlated to overcome the decrease in intensity. A Levenberg-Marquardt non linear least-square algorithm is used to fit the data.

\section{FCS measurements in living neurons}

The sample containing living neurons in Tyrode's solution is mounted on the sample stage of the microscope. Measurements are performed on mature hippocampal cultured neurons expressing EGFP-synapsin, mEGFP, membrane EGFP and mEGFP-SNAP25. Data are acquired from different positions along axons for each protein analyzed. Each position is measured at least 20 times, with acquisition times between $10 \mathrm{~s}$ to $30 \mathrm{~s}$ for each round of acquisition. All of the data are then fitted with a Levenberg-Marquardt non linear least-square routine using a self-written Python code. Data, in which large fluorescence peaks are observed or with pronounced photobleaching, are excluded from the analysis. The cell culture, transfection and manipulation was carried out by our collaborator Sofiia Reshetniak from the University Medical Center of Göttingen in the Institute for Neuro- and Sensory Physiology. 


\section{PCH experiments}

The data for each PCH are collected for a time duration between $300 \mathrm{~s}$ and $800 \mathrm{~s}$. To decrease the computational time, the data are acquired in slots of $10 \mathrm{~s}$ each and then combined together, excluding the runs where aggregates were present. The acquired photon arrival times are binned with a binning time between 2 and $20 \mu \mathrm{s}$, stored and used to build the experimental PCH curves. A histogram of the counts per bin, $k$, is created and normalized. The experimental $\mathrm{PCH}, p(k)$ is fitted with the theoretical model described in [8] using a Levenberg-Marquardt non linear least-square algorithm. All the scripts used during the $\mathrm{PCH}$ calculation and analysis are custom written with Python code. The OPE correction parameter F for our experiment is fixed to 0.6 and the dead-time is measured to be $86 \pm 19$ $\mathrm{ns}$ (45 $\mathrm{ns}$ is the value provided the manufacturer).

\subsection{Finite element methods simulations}

In Section 2.2, an introduction to the governing equations of fluid mechanics is presented. However, a simple analytical solution for the fluid mechanic equations exists only in very exceptional cases, for all the other cases numerical simulations have to be performed to approximate a solution [71]. Here, FEM simulations are performed using COMSOL Multiphysics 5.2a (COMSOL GmbH, Göttingen, Germany). In this method the object to simulate is discretized in a tetraedic mesh, as shown in Figure 3.8. Transport equations and Navier-Stokes equation are approximated and solved for every component of the mesh. The mesh dimension is not uniform but it is adapted to the object to simulate. Simulations are performed using the time independent Navier-Stokes equation with no slip boundary condition. To decrease the computational time, the simulated channels are shorter and only a quarter of the device is simulated (Figure 3.8) with imposed symmetry conditions. The simulations of vimentin assembly are performed using an upper limit diffusion coefficient for vimentin $\left(D_{\text {vim }}=22 \mu \mathrm{m}^{2} / \mathrm{s}\right)$. The sheath buffer is simulated as water while $D_{\mathrm{KCl}}=1850 \mathrm{\mu m}^{2} / \mathrm{s}$ is used for the assembly buffer. The concentration and the velocities used in the simulations are equal to the experimental conditions $\left(c_{\mathrm{vim}}=0.0025 \mathrm{~g} / \mathrm{L}\right.$ and $c_{\mathrm{KCl}}=100 \mathrm{mM}$, and $Q=12 \mu \mathrm{L} / \mathrm{h}$ for the central inlet, $Q=10 \mu \mathrm{L} / \mathrm{h}$ for the diagonal inlets and $Q=190 \mu \mathrm{L} / \mathrm{h}$ for the side inlets). The change in viscosity along the channel is not included in the simulations, since viscometry measurements show that the viscosity does not significantly increase during the first assembly steps [119, 122].

The simulations for the device used with patterned SVs are performed using the diffusion coefficient of water in the central inlet and the diffusion coefficient of fluorescein for the side inlets $\left(425 \mathrm{\mu m}^{2} / \mathrm{s}\right)$. The velocity in the central inlet is fixed to $0.1 \mathrm{~mm} / \mathrm{s}$ while in the side inlets the velocities over time are defined as a periodic rectangular function $(\max 1 \mathrm{~mm} / \mathrm{s}, \min 0.1 \mathrm{~mm} / \mathrm{s}$ and width $10 \mathrm{~s})$, as shown in Figure 4.38 a (Section 4.2.3). The velocity functions have a periodicity 


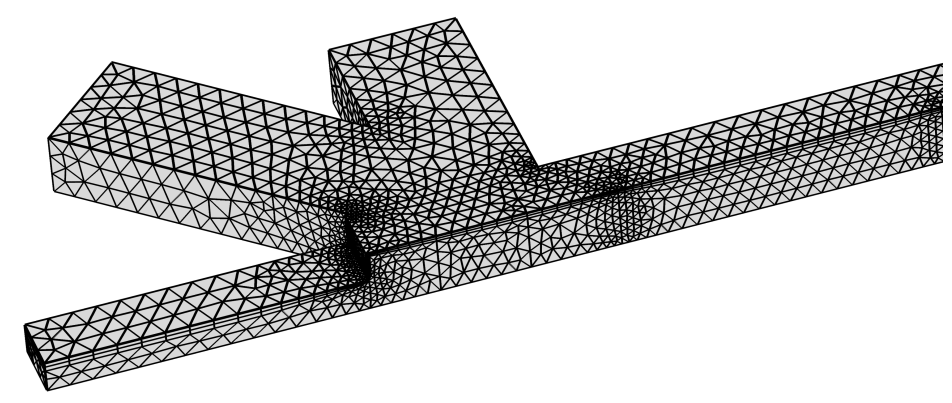

Figure 3.8: First section of the geometry of the channel used for a FEM simulation. A quarter of the microfluidic device (step device with 5 inlets) is simulated to reduce the computational time. The tetraedic mesh is shown. Here the mesh has a coarse size ( $\max$ size $36 \mu \mathrm{m}$ and min size $7 \mu \mathrm{m}$ ) for visualization purposes. In reality, a much smaller mesh is used to simulate the channel (maximum size $7 \mu \mathrm{m}$ and minimum size $0.0007 \mu \mathrm{m}$ ). The local adaptation of the mesh is visible at the inlet junctions.

of $20 \mathrm{~s}$ and they are shifted in time, to achieve a constant flow velocity in the center of the channel of $1.2 \mathrm{~mm} / \mathrm{s}$. 
$\Gamma_{\text {Chapter }}$

Results

In this chapter, the main results of the experiments conducted will be presented. First, the experiments on vimentin assembly are presented. The combination of a specific microfluidic device and FFS techniques allows us to measure the early time points of vimentin assembly. The outcomes of these experiments will be described and discussed in relation to the current scientific results. Second, the experiments conducted on live neurons and SVs, are presented. Diffusion and interactions of synaptic proteins are measured, using FCS, in living neurons and on patterned SVs. These experiments are carried out in collaboration with Sofiia Reshetniak and Prof. Silvio Rizzoli from the Institute for Neuro- and Sensory Physiology in University Medical Center of Göttingen. A third microfluidic approach is employed to achieve a controlled exchange of buffer on the patterned SVs. This project is in collaboration with Helena Maria Olsthoorn and Prof. Reinhard Jahn from the Laboratory of Neurobiology of the Max Plank Institute for Biophysical Chemistry in Göttingen.

\subsection{Studying protein interactions and labeling stoichiome- try under flow}

The main subject of this section is the study on the early temporal steps of vimentin assembly, which are measured with a combination of FFS techniques and microfluidics. The microfluidic devices used and the experimental results are presented and discussed within the framework of vimentin assembly. The combination of microfluidics and FFS presented here is particularly interesting, because it can also be applied to study the aggregation of other molecules. For example, it can be employed to study the assembly of proteins with fast assembly dynamics, like keratin, or to study proteins in a controlled environment that could be important for a "chameleon" protein, like $\alpha$-synuclein. 


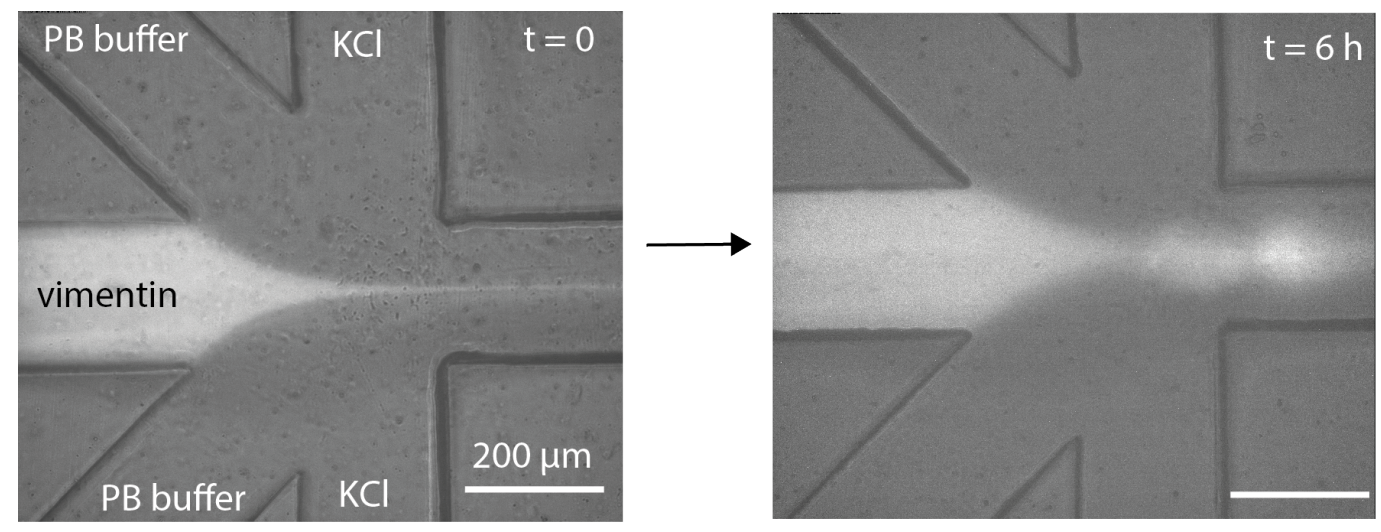

Figure 4.1: Vimentin assembling in flow inside a five-inlet microfluidic device with the same height for all channels (i.e. no-step device). At the beginning of the measurement, the vimentin stream is clearly focused by the side inlets. After 6 hours of assembly, protein aggregates are formed in the device. Since some of our measurements can last up to 9 hours, the aggregation of vimentin in the device is an important factor to take into consideration. Images courtesy of Manuela Denz.

\subsubsection{Microfluidic device optimization}

The microfluidic device is essential for this experiment because it gives access to the early temporal points of the assembly. The device acts as a mixer, it brings in contact vimentin and assembly ions. We can assume a laminar flow regime as the $R e$ is lower then the critical value (with maximum velocity $\approx 5 \mathrm{~mm} / \mathrm{s}$, $L=200 \mu \mathrm{m}$ and $\eta=0.9 \mathrm{mPas}, R e \approx 100$ ). The mixing between vimentin and $\mathrm{KCl}$ ions happens only via diffusion. The Péclet number quantifies the distance along the channel, in terms of the channel width, after which the mixing of two solutions is complete. If $P e$ is small, diffusion dominates the transport in the device. If $P e$ is big, the solute follows the flow lines. As described in Section 2.2 .3 , in a microfluidic device, protein transport is mostly convectional, while diffusion dominates the transport for ions. For a protein with diffusion coefficient in the order of $10^{-11}$ with a $1 \mathrm{~mm} / \mathrm{s}$ velocity and $\omega=100 \mu \mathrm{m} P e$ is around $10^{4}$, while for an ion with diffusion coefficient in the range of $10^{-9} \mathrm{Pe}$ is around 100 . In this case the ion will diffuse along the width of the channel faster than the protein molecules. To reduce the mixing times [163] of the ions in the vimentin stream, the protein is focused by the side inlets.

To achieve a controlled assembly starting point, a five-inlets geometry is chosen, where the diagonal inlets prevent the mixing of the ion solution before the focusing of the stream [75]. Since in laminar flow regime there are no turbulences, the spatial coordinate of each measured position can be directly converted, once the velocity is known, in the time coordinate of the assembly process. Moreover, the laminar flow regime ensures that molecules measured at the same position 


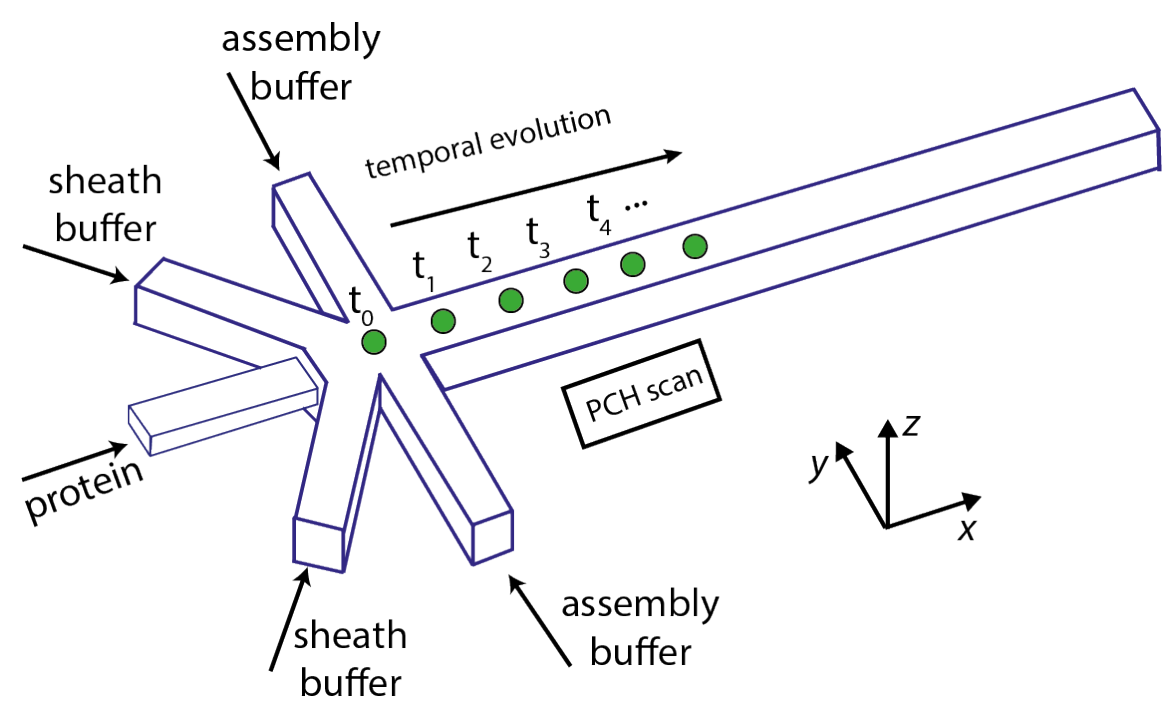

Figure 4.2: Sketch of the step microfluidic device (width $=200 \mu \mathrm{m}$, height $=100 \mu \mathrm{m}$ for the central inlet and $200 \mathrm{um}$ for the remaining channels) employed to study the assembly of vimentin. Vimentin assembly starts at $110 \mu \mathrm{m}$ in the $x$-direction, from the "step" in the center channel. From there, $\mathrm{PCH}$ measurements are acquired in the center of the outlet channel (green dots).

in the channel find themselves in the same temporal step of the assembly. This means, that even "slow" techniques, such as PCH, can be used to measure fast kinetics. The time resolution of the process is constrained by the microscope stage movement and the beam size, but not by the technique used.

Originally, a five-inlet geometry with the same channel height everywhere, i.e. with no step, was tested. However, it has been shown that assembling vimentin molecules in microfluidic devices with uniform channel height tend to stick to the channel walls. Figure 4.1 shows an experiment of vimentin assembly under flow. Vimentin, after some hours from the starting of the experiment, starts to create aggregates in the channel that, with time, will clog the device. To avoid the adsorption of protein, passivation with BSA and Poloxamer 407 has been tried without any success.

Figure 4.2 , shows a sketch of the five-inlet device used during the experiments. Examples of the measured positions are depicted as the green dots along the outlet. A width of $200 \mu \mathrm{m}$ is used for all the channels. A height of $100 \mu \mathrm{m}$ is used for all the channels apart from the central channel where a height of $100 \mu \mathrm{m}$ is employed. This device will be called from here on, step device. Instead of using a chemical to passivate the surface, a "physical passivation" is used to prevents vimentin from touching the channel walls [14]. The idea is to have a protection layer of buffer solution that engulfs the vimentin stream in the cross section of the device, preventing any contact, not only with the side walls, but also with the top and the bottom channel surface. To create the protection layer, the height of the central stream is halved compared to the other channels and flow rates have 

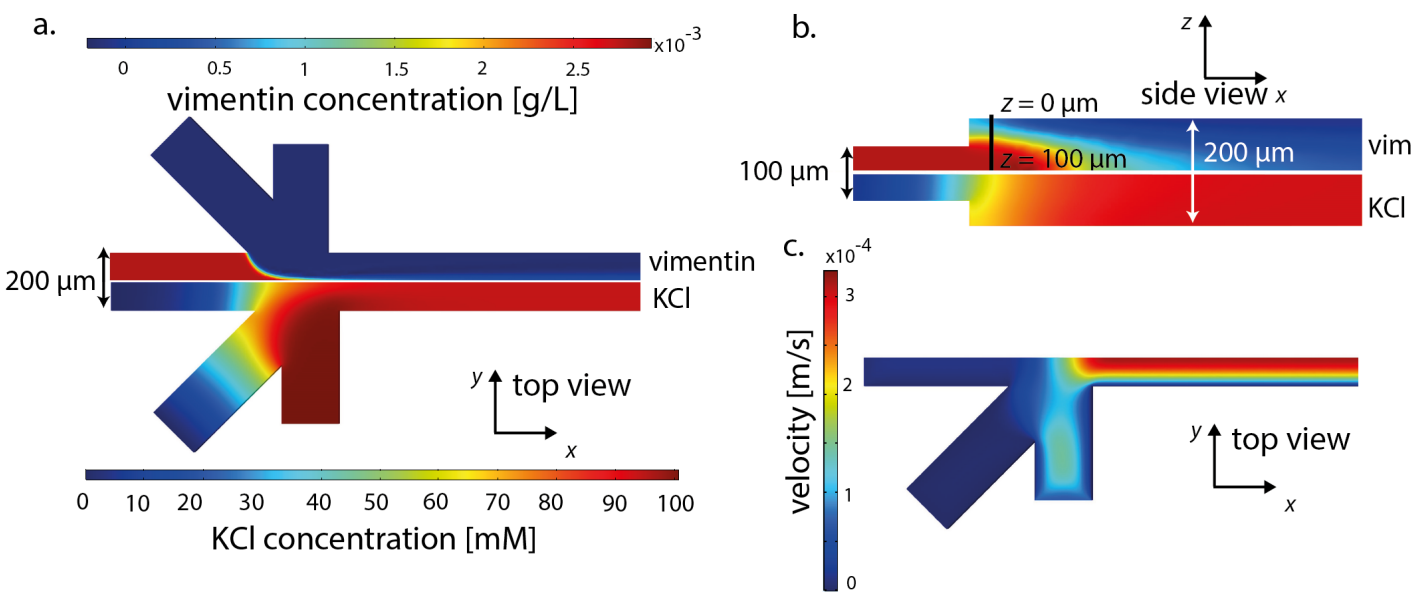

Figure 4.3: Result of a FEM simulation for a five-inlet microfluidic device (width $=200 \mu \mathrm{m}$, height $=100 \mu \mathrm{m}$ for the central inlet and $200 \mu \mathrm{m}$ for the remaining channels) with vimentin tetramers at slow flow rates. Just a quarter of the microfluidic device is simulated. (a) Top view of vimentin concentration and $\mathrm{KCl}$ concentration. (b) Side view of the central section of the microfluidic device. Vimentin tetramers are in contact with the channel walls (top part). (c) Flow velocity in the channel. The maximum value of the velocity is $\approx 300 \mu \mathrm{m} / \mathrm{s}$. This simulation is performed with a flow velocity of $7 \mu \mathrm{m} / \mathrm{s}$ for the central inlet, $2 \mu \mathrm{m} / \mathrm{s}$ for the diagonal inlets and $65 \mathrm{~m} / \mathrm{s}$ for the side inlets.

to be adjusted in a way that the flow from the side inlets can actually surround the protein stream.

FEM simulations provide a tool to find out the perfect flow parameters. Figure $4.3 \mathrm{a}-\mathrm{b}$ shows the concentration of vimentin tetramers in the cross section of the step device simulated with a set of slow flow rates. The maximum flow velocity in the central channel is around $300 \mu \mathrm{m} / \mathrm{s}$ as shown in Figure $4.3 \mathrm{c}$, in particular, $7 \mu \mathrm{m} / \mathrm{s}$ is used for the central inlet, $2 \mu \mathrm{m} / \mathrm{s}$ for the diagonal inlets and $65 \mu \mathrm{m} / \mathrm{s}$ for the side inlets. These set of flow rates are chosen because these are the velocities to use if FCS is the technique of choice to measure the assembly. As explained in Section 2.1.2, FCS can be applied to measure aggregation even when molecules are under flow, however the flow must be slow enough to still be able to measure diffusion. It has been shown that when the ratio of the flow time, $\tau_{\mathrm{F}}=\frac{w_{0}}{v}$ (with $w_{0}$ being the diameter of the observation volume in the radial direction), and the diffusion time, $\tau_{D}=\frac{w_{0}^{2}}{4 D}$, is smaller than 0.02 , flow dominates over diffusion [53]. As a first approximation, the flow time $\tau_{\mathrm{F}}$ has to be at least the same value as the diffusion time $\tau_{D}$ to be able to measure diffusion. If vimentin tetramers are considered, with a diffusion coefficient of $D=22 \mathrm{\mu m}^{2} / \mathrm{s}$ in an observation volume with a diameter of $w_{0}=300 \mathrm{~nm}, \tau_{D}$ is around $1024 \mu \mathrm{s}$. Then, the maximum velocity allowed is $\approx 300 \mu \mathrm{m} / \mathrm{s}$, which is similar to the maximum velocity in the center of the device in the simulation with slow flow rates (Figure 4.3). Vimentin assemblies, such as ULFs, have even lower diffusion coefficients, limiting the ve- 
a.
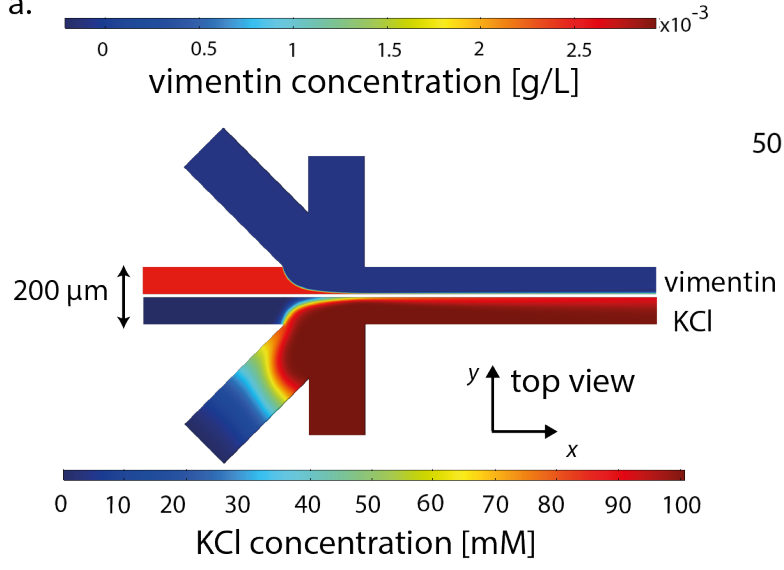

b.
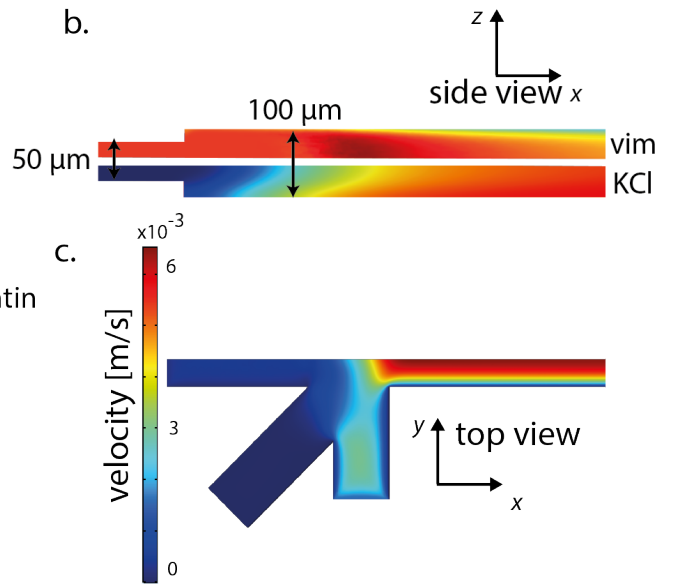

Figure 4.4: Results of a FEM simulation for the step device B, with a width of $200 \mu \mathrm{m}$, a height of $50 \mu \mathrm{m}$ for the central inlet and $100 \mu \mathrm{m}$ for the remaining channels. (a) Top view of vimentin concentration and $\mathrm{KCl}$ concentration. (b) Side view of the central section of the microfluidic device. Vimentin tetramers are in contact with the channel walls (top part). (c) Flow velocity in the channel. The maximum value of the velocity is $\approx 6 \mathrm{~mm} / \mathrm{s}$. Despite the higher flow rates respect the simulation with slow flow rates (set of high flow rates: $1.35 \mathrm{~mm} / \mathrm{s}$ for the side inlets, $0.15 \mathrm{~mm} / \mathrm{s}$ for the central inlet and $0.07 \mathrm{~mm} / \mathrm{s}$ ), the geometry of the device prevents the creation of the buffer protection layer, thus vimentin molecules are in contact with the channel walls.

locity to smaller values. If, for example, a diffusion coefficient around $5 \mathrm{\mu m}^{2} / \mathrm{s}$ is considered, $\tau_{D}$ is $\approx 4500 \mu \mathrm{s}$, consequently the maximum flow velocity has to be $60 \mu \mathrm{m} / \mathrm{s}$.

However, with the slow flow rates, even if there is the step in the device, vimentin tetramers are still in contact with the channel walls, as shown in Figure 4.6 (orange line), where at $z=0$, i.e. at the channel wall, the concentration is still not zero. This is due to the slow flow rates used for this simulation. Here, the fluid from the side inlets is not enough to surround the central stream and create the protection layer. A previous $\mathrm{PhD}$ student in our group, Dr. Viktor Schroeder, actually tried to measure assembly of vimentin using FCS [130]. However, he concluded that with the restrictions imposed on the flow velocities by the microfluidic device and the limitations of FCS in flow, it is impossible to combine the two techniques.

Other geometries were also simulated. A device with a channel width of $200 \mu \mathrm{m}$ and height of $50 \mu \mathrm{m}$ for the central inlet, and $100 \mu \mathrm{m}$ for the other channels, (called step device B) has been simulated using a set of high flow rates. The velocities used here are higher than the ones used in the simulation for the step device at slow flow rates $(1.5 \mathrm{~mm} / \mathrm{s}$ for the side inlets, $0.3 \mathrm{~mm} / \mathrm{s}$ for the central inlet and 0.1 $\mathrm{mm} / \mathrm{s}$ for the diagonal inlets). Also in this case, vimentin is still in contact with the channel walls, as shown in Figure $4.4 \mathrm{~b}$ and in Figure 4.6 (blue line). Thus, also the geometry of the device plays an important role in preventing vimentin 


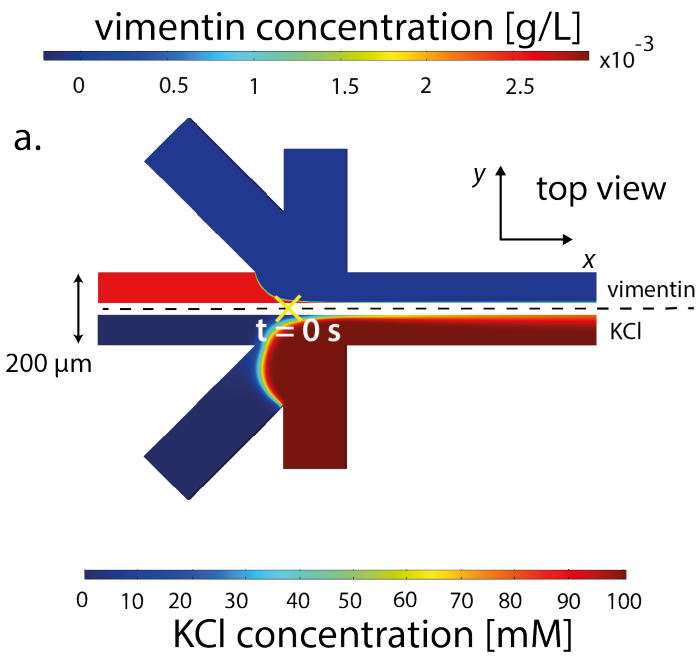

b.

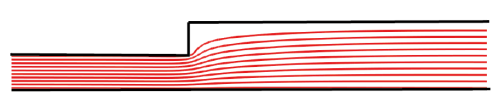

c.

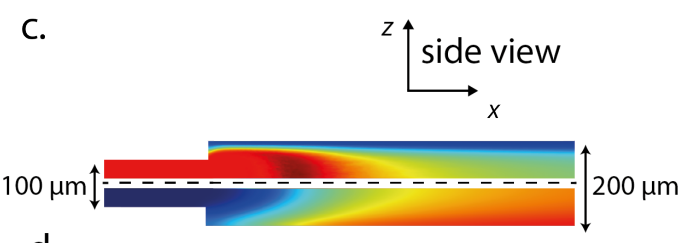

d.

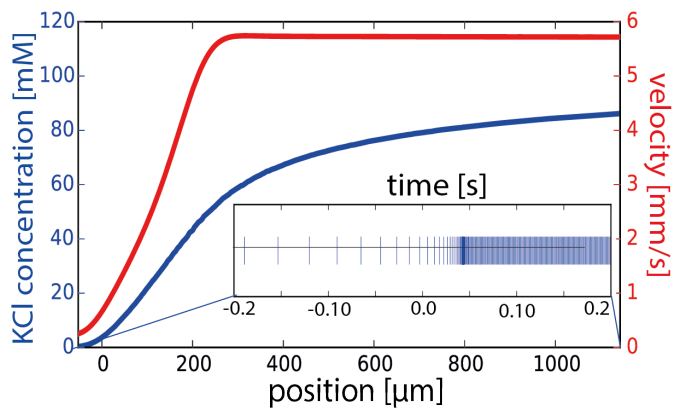

Figure 4.5: Results for a FEM simulation of the step microfluidic device (width $=200 \mu \mathrm{m}$, height $=100 \mu \mathrm{m}$ for the central inlet and $200 \mu \mathrm{m}$ for the remaining channels) used to study vimentin assembly. The flow velocities used during the simulations are the experimental ones (set of high flow rates). (a) Top view of the vimentin tetramers concentration and of the $\mathrm{KCl}$ buffer concentration. At $110 \mu \mathrm{m}$ from the end of the central inlet the assembly starts. In this position the concentration of $\mathrm{KCl}$ reaches the threshold to start the assembly of vimentin $(10 \mathrm{mM})$. This position is set to be time 0. (b) Side view of the flow streamlines. (c) Side view of vimentin and $\mathrm{KCl}$ concentration in the cross section of the device. Note how vimentin concentration is 0 at the channel wall. (d) $\mathrm{KCl}$ concentration and velocity magnitude in the central channel. Since the velocity is not constant at the beginning of the device, the calculated reaction time is not uniformly spaced. Negative time points corresponds to positions before the $\mathrm{KCl}$ threshold concentration of $10 \mathrm{mM}$ is reached.

to stick to the channel walls.

If the step device is simulated with the high flow rates set $(1.35 \mathrm{~mm} / \mathrm{s}$ for the side inlets, $0.15 \mathrm{~mm} / \mathrm{s}$ for the central inlet and $0.07 \mathrm{~mm} / \mathrm{s}$ for the diagonal inlets), vimentin molecules are finally not in contact anymore with the channel walls as shown in Figure 4.5. The flow velocities used here, combined with the constriction of the central channel, allow for the creation of the buffer protection layer around the central stream, preventing vimentin from touching the channel walls. In Figure $4.5 \mathrm{~b}$, the vimentin streamlines are shown at the cross section of the step device (side view). The lines are not in contact with the channel walls. The concentration of vimentin is 0 at the channel walls in the cross section of the device, as shown in Figure 4.5. Figure 4.5 a shows the top view of vimentin concentration and $\mathrm{KCl}$ concentration inside the device.

The ions of the assembly buffer in the side inlets diffuse into the vimentin 


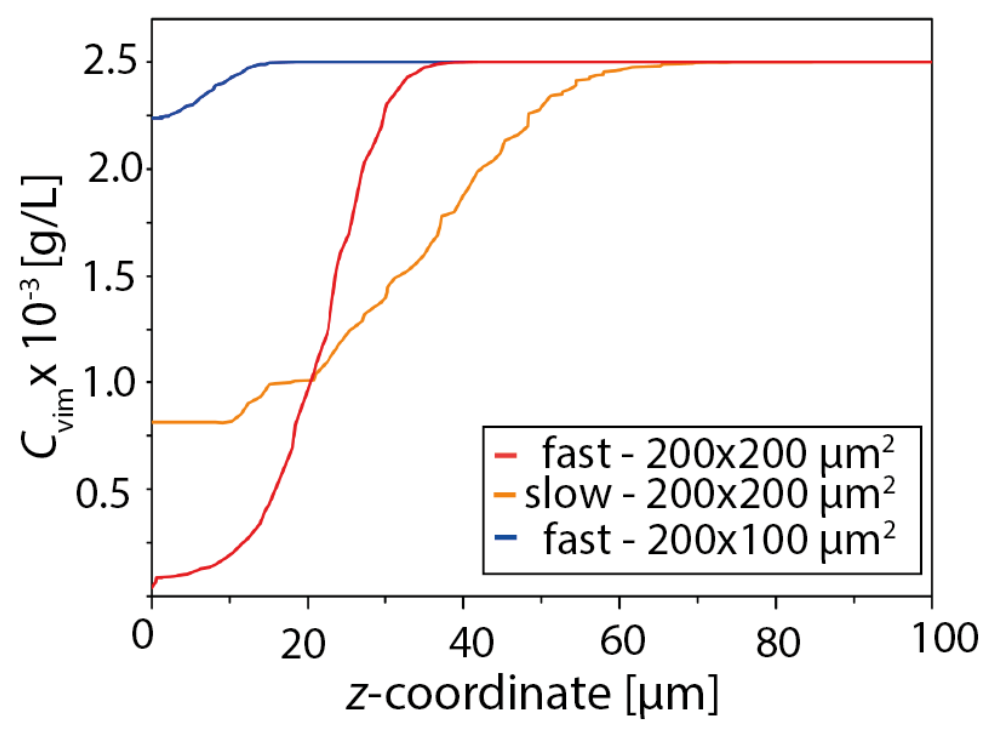

Figure 4.6: Comparison of vimentin concentration along $z$ for the three microfluidic devices simulated. The concentration of vimentin tetramers is plotted along the $z$-axis at $10 \mu \mathrm{m}$ from the end of the central inlet in the $x$-axis. The lowest concentration at the channel wall $(z=0)$ is for the step device (width $=200 \mu \mathrm{m}$ and height $=100 \mu \mathrm{m}$ for the central channel and $200 \mu \mathrm{m}$ for the remaining channels) simulated with the fast flow rates.

protein stream starting the assembly. The trigger concentration of $\mathrm{KCl}$ buffer to start the assembly is $10 \mathrm{mM}$ [87. This value is reached after $110 \mu \mathrm{m}$ from the end of the central inlet, this position is set to be the origin of the $x$-axis. The trigger position is calculated from the line plot of the $\mathrm{KCl}$ concentration along the outlet (Figure 4.5d). The velocity inside the device increases when the solutions from all the inlets meet. At $\approx 200 \mu \mathrm{m}$ from the origin the velocity has a constant value of $5.7 \mathrm{~mm} / \mathrm{s}$ This velocity is too high for FCS measurements, therefore PCH is used as an alternative technique. The limitations on the device geometry and the flow rates are part of the reason that convinced us to use PCH instead of FCS. PCH is also more sensitive to aggregation processes: it can distinguish two molecules if the brightness differs at least a factor of 2 in brightness, thus, in principle if every monomer has one label, the dimer formation can be followed. On the contrary, the formation of a dimer leads to an increase in $D$ of a factor of 1.26 which is be difficult to measure with FCS in flow. Since PCH is not dependent on the flow rates there is no limitation on the velocities chosen, on the condition that undersampling is avoided, as explained in Section 2.1.3. The sampling time $T$ has to be faster than the fluorescence fluctuation time scales. With the set of high flow rates, the maximum velocity in the center of the microfluidic devices used here leads to a flow time $\tau_{\mathrm{F}}$ of $50 \mathrm{\mu s}$. The binning time used for all the PCH measurements in flow is $5 \mu \mathrm{s}$. Since the velocity is not constant along the central 


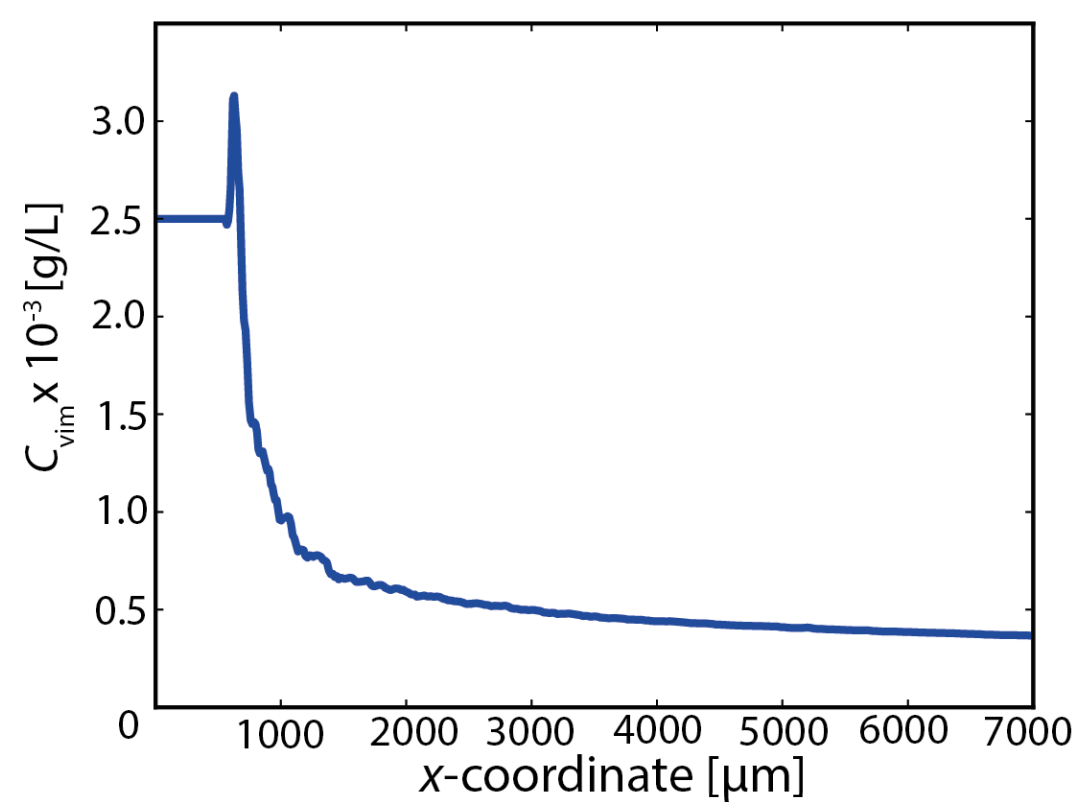

Figure 4.7: Vimentin concentration along the center of the microfluidic device. The concentration decreases along the device due to the dilution caused by the flow coming from the other inlets. The kink in the vimentin concentration, at $x=600 \mu \mathrm{m}$, is an artifact due to the finite size of the calculated mesh in the FEM simulation.

channel, the calculated reaction time is not uniformly spread. Time 0 is set to be the $x$ position where $\mathrm{KCl}$ concentration is $10 \mathrm{mM}$ in the center of the device [15]. Positions before this threshold are associated with negative time, as showed in the inset of Figure $4.5 \mathrm{~d}$. When the velocity is increasing, the calculated time points are closer to each other, as soon as the velocity reaches a constant value, the time points are uniformly spread along the outlet positions. While the $\mathrm{KCl}$ concentration increases along the center of the outlet, reaching a plateau of 100 $\mathrm{mM}$ at $\approx 1.5 \mathrm{~mm}$ from the origin, the vimentin concentration decreases along the outlet, as shown in Figure 4.7. In the simulations, the decrease of vimentin concentration is only to be attributed at the dilution of the vimentin stream by the other inlets, as the assembly reaction is not simulated.

To summarize, the geometry chosen to measure the assembly of vimentin in flow is a five-inlet and one outlet microfluidic device with a channel width of $200 \mu \mathrm{m}$ for all the channels and an height of $100 \mu \mathrm{m}$ for the central channel and of $200 \mu \mathrm{m}$ for the remaining channels (step device). The flow velocities applied in the experiments are equal to the simulated ones (set of high flow rates: 0.15 $\mathrm{mm} / \mathrm{s}$ for the central inlet, $1.35 \mathrm{~mm} / \mathrm{s}$ for the side inlets and $0.07 \mathrm{~mm} / \mathrm{s}$ for the diagonal inlets). 


\subsubsection{PCH measurements of vimentin tetramers in bulk}

In this section, $\mathrm{PCH}$ measurements and simulations of vimentin tetramers are presented. The simulations are used to test the self-written $\mathrm{PCH}$ analysis code and to find the optimal vimentin labeling ratio to use during the assembly experiments. Afterwards, $\mathrm{PCH}$ measurements of vimentin tetramers in bulk are discussed.

\section{Optimization of the PCH experiments to study vimentin assembly}

$\mathrm{PCH}$ measurements of vimentin tetramers in bulk are acquired to test the method on our setup. Ideally, for studying molecular aggregation with $\mathrm{PCH}$, exactly one label is attached to each molecule. In this way, once the brightness of a single molecule is known, the aggregation can be followed measuring the brightness of the aggregates. A dimer would have a brightness twice the value of the single molecule, a trimer three times the value and so on. However, if one dye molecule is attached to one vimentin monomer, the assembly does not work anymore [114]. In fact, even if the dye molecule is attached to the tail domain of vimentin, it prevents the full lateral and longitudinal assembly for sterical reasons. A labeling ratio between $25 \%$ and $36 \%$ is typically used here.

The distribution of labels in a vimentin sub-unit is given by the binomial distribution. The probability to have $k$ labeled molecules in a polymer made of $n$ monomers is:

$$
p_{n}^{k}=\left(\begin{array}{l}
n \\
k
\end{array}\right) r^{k}(1-r)^{n-k} .
$$

With this equation, knowing the initial labeling ratio, defined as the percentage of the ratio between labeled vimentin monomer and labeled + unlabeled vimentin monomers, it is possible to calculate the expected average number of labels for vimentin sub-unit at each stage of the assembly. For example, to calculated the average number of label for a vimentin tetramer, we have to take into consideration the probability of having $0,1,2,3$ or 4 fluorophores ( 4 is the maximum number of dye molecules attached because 4 vimentin monomers are assembled into one tetramer) attached to each tetramer. If the labeling ratio is $40 \%$, the probability of having $0,1,2,3$ or 4 fluorophores is calculated with equation 4.1 , in particular, for the case of 1 fluorophore per tetramer, equation 4.1 is $p_{4}^{1}=\left(\begin{array}{l}4 \\ 1\end{array}\right) 0.40^{1}(1-0.40)^{4-1}$. Then, to obtain the average number of fluorophores for the tetramer $\left(N_{\text {dye/4 }}\right)$, each probability is multiply with the related number of labels and the sum of all the resulting values is divided by the sum of the binomial probabilities excluding the 0 -fluorophore case $\left(N_{\text {dye } / 4}=\left(p_{4}^{0} \cdot 0+p_{4}^{1} \cdot 1+p_{4}^{2} \cdot 2+p_{4}^{3} \cdot 3+p_{4}^{4} \cdot 4\right) /\left(p_{4}^{1}+p_{4}^{2}+p_{4}^{3}+p_{4}^{4}\right)\right)$.

To test if is possible to distinguish vimentin molecules during the assembly with PCH at labeling ratios between $10 \%$ and $33 \%$, simulations are carried out using the software Global Images-SimFCS4 developed by the group of Prof. Enrico Gratton (Laboratory for Fluorescence Dynamics, USA). The software simulates movements of fluorescence molecules and their acquisition through a microscope. 

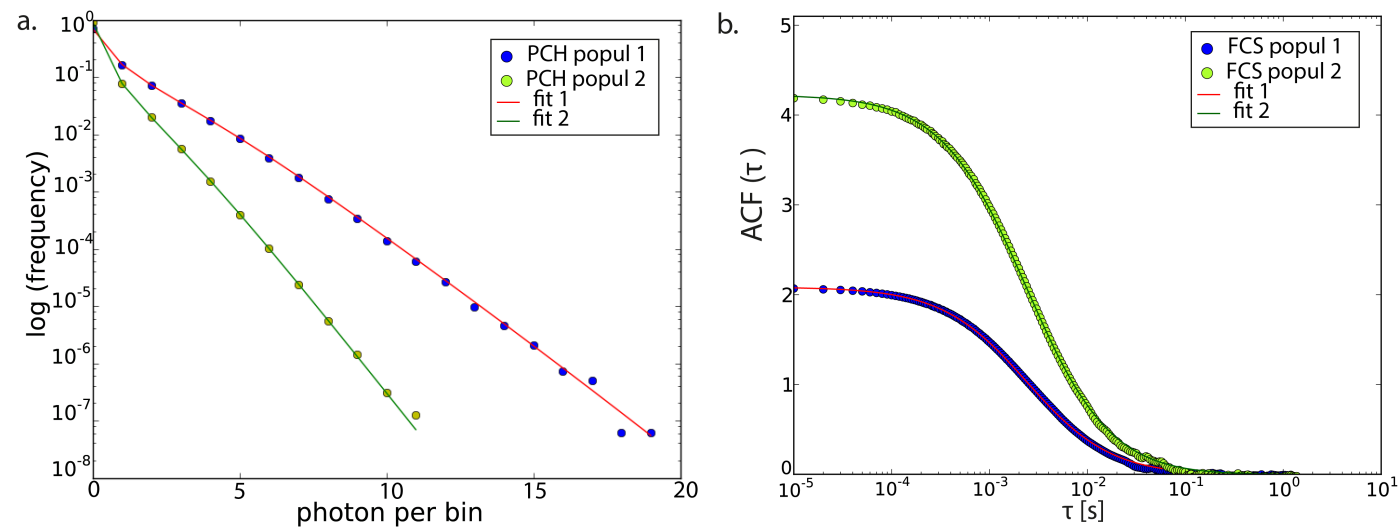

Figure 4.8: PCH and FCS simulation results and fits. The dots represent the simulated data, the lines are the fits analyzed with the custom written code. Population 1 represents 100 particles diffusing with $D=10 \mathrm{\mu m}^{2} / \mathrm{s}$ and $B=100000 \mathrm{cps}$. Population 2 represents 50 particles diffusing with $D=10 \mathrm{~mm}^{2} / \mathrm{s}$ and $B=50000 \mathrm{cps}$.

The acquired data can be analyzed with the same software. It can be used to simulate the fluorescence signal from Brownian motion or convective flow of fluorescence particles. Initially, we use it to test the validity of the self-written $\mathrm{PCH}$ fitting algorithm.

For this simulation, two populations of particles diffusing in a box are simulated separately in the confocal acquisition mode. First, 100 particles diffusing in the box $\left(D=10 \mathrm{\mu m}^{2} / \mathrm{s}\right)$ with a brightness $(B)$ of $100 \mathrm{kcps}$, (population 1 ) are simulated. Subsequently, in another simulation, 50 particles with a brightness of 50 kcps (population 2) are simulated with the same box size, acquisition parameters and diffusion coefficient. Counts per second or cps is considered in the field, the unit of brightness. The fluorescence signal from the diffusing molecules is acquired for $163 \mathrm{~s}$ and then $\mathrm{ACF}$ and $\mathrm{PCH}$ are built from the acquired intensity. In Figure 4.8, the dots represent the $\mathrm{PCH}$ of the simulated data, blue for population 1 and light green for population 2. The lines are the fitting curves obtained with the self-written algorithm. The model to fit the data is the one component PCH model, described by equation 2.29 . The parameters obtained from the fit are the brightness of the measured molecule and the average number of particles, with that brightness, in the observation volume. Both FCS and PCH are also analyzed with SimFCS software. The fitting parameters, summarized in Table 4.1, are in good agreement with each other.

As expected, the brightness of one molecule from population 2 is half the value of the molecular brightness from one molecule of population 1 . The value of the fitted brightness represents the brightness emitted from each molecule during one time bin $(10 \mu \mathrm{s})$. Also, the number of molecules per observation volume scales according to the initial values. The average number of molecules in the obser- 
594.1. Studying protein interactions and labeling stoichiometry under flow

\begin{tabular}{ccccccc}
\hline Population & $b_{\text {SimFCS }}$ & $b_{\text {self }}$ & $N_{\text {SimFCS }}$ & $N_{\text {self }}$ & $N_{F C S}$ & $D\left[\mathrm{\mu m}^{2} / \mathrm{s}\right]$ \\
\hline \hline 1 & 3.39 & 3.44 & 0.16 & 0.16 & $0.1671 \pm 2 \times 10^{-4}$ & $9.17 \pm 0.04$ \\
\hline 2 & 1.67 & 1.68 & 0.08 & 0.08 & $0.0830 \pm 1 \times 10^{-4}$ & $9.33 \pm 0.04$ \\
\hline \hline
\end{tabular}

\begin{tabular}{ll|l}
\hline Table 4.1: Fitting results from the analysis of the simulated data shown in Figure 4.8 & The
\end{tabular} parameters computed with the custom code are in good agreement with the results from the SimFCS software. The parameters obtained from the FCS analysis are comparable with the PCH parameters, same $N$ values and same diffusion coefficients for both curves.

\begin{tabular}{ccccc}
\hline $\begin{array}{c}\text { LR } \\
\%\end{array}$ & $\begin{array}{c}B_{4 m e r} \text { (No.dye) } \\
{[\mathrm{kcps}]}\end{array}$ & $\begin{array}{c}B_{8 m e r} \text { (No.dye) } \\
{[\mathrm{kcps}]}\end{array}$ & $\begin{array}{c}B_{16 m e r} \text { (No.dye) } \\
{[\mathrm{kcps}]}\end{array}$ & $\begin{array}{c}B_{32 m e r} \text { (No.dye) } \\
{[\mathrm{kcps}]}\end{array}$ \\
\hline \hline 10 & $37.6(1.16)$ & $45.4(1.40)$ & $63.5(1.96)$ & $107.2(3.3)$ \\
\hline 25 & $47(1.46)$ & $72(2.22)$ & $130(4.04)$ & $259(8.00)$ \\
\hline 33 & $53(1.66)$ & $89(2.77)$ & $172(5.33)$ & $344(10.65)$ \\
\hline
\end{tabular}

Table 4.2: Table summarizing the brightnesses and the numbers of label per vimentin molecule (in brackets) at different assembly stages for three different labeling ratios.

vation volume agrees well also with the FCS analysis. The diffusion coefficients obtained from the fits are in agreement with the input value in both simulations.

SimFCS is used to test vimentin samples with different labeling ratios at different assembly stages. As already introduced, the labeling ratio should be as low as possible to allow for a normal assembly reaction but high enough to allow for distinguishing the various components during vimentin assembly. Since PCH can distinguish populations when they differ in brightness by a factor of two, we test which labeling ratio allows us to distinguish at best the vimentin sub-units during the different stages of the assembly. For this assessment, two different populations of particles with the brightness of vimentin sub-units, are simulated diffusing together in the same box. Three labeling ratios are tested: 10\%, 25\% and $33 \%$. Higher labeling ratios are not taken into consideration because vimentin would not be able to form filaments. In Figure 4.9 two batches of vimentin, one with a LR of $25 \%$ and one with a LR of $100 \%$, are shown after 7 hours of assembly at $37^{\circ} \mathrm{C}$. When the $\mathrm{LR}$ is $100 \%$ vimentin filaments are not formed, possibly due to the high density of the attached fluorophores which inhibits the assembly.

The brightness of each component, tetramer, octamer, hexadecamer and ULF, is estimated, calculating the number of labels per vimentin sub-unit, using equation 4.1. The obtained number of label is multiplied with the brightness of a single Alexa-532 molecule, which was measured, $B \approx 33 \times 10^{3}$ cps. In Table 4.2 . the calculated numbers of labels and the brightnesses used in the simulations are summarized for the different labeling ratios.

As an example, the simulation for tetramers and ULFs diffusing together is reported. In Figure 4.10 the simulated PCH data are shown together with the fits 


\section{Vimentin after 7 hours of assembly}
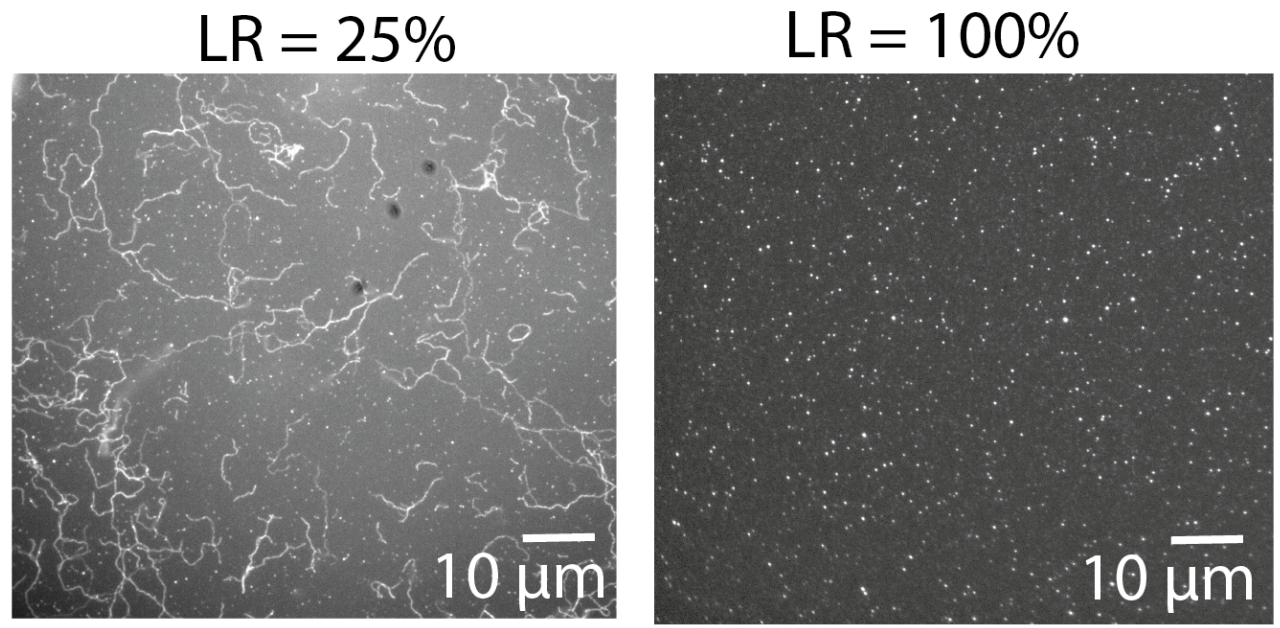

Figure 4.9: Epi-fluorescence images of vimentin filaments with different LRs after 7 hours of assembly at $37^{\circ} \mathrm{C}$. When the LR is $25 \%$ filaments are properly formed. At LR $=100 \%$ no vimentin filaments are visible in the sample.

\begin{tabular}{ccccc}
\hline LR [\% $]$ & $B_{4 m e r}($ fit) $[\mathrm{kcps}]$ & $B_{32 m e r}($ fit $)[\mathrm{kcps}]$ & $N_{4 m e r}($ fit $)$ & $N_{32 m e r}($ fit $)$ \\
\hline \hline 10 & $37 \pm 128$ & $106 \pm 20$ & $0.2 \pm 0.7$ & $0.2 \pm 0.2$ \\
\hline 25 & $57 \pm 11$ & $259 \pm 2$ & $0.24 \pm 0.05$ & $0.233 \pm 0.003$ \\
\hline 33 & $53 \pm 10$ & $338.9 \pm 0.8$ & $0.2 \pm 0.1$ & $0.248 \pm 0.002$ \\
\hline
\end{tabular}

Table 4.3: Brightness $(B)$ and number of molecules per observation volume $(N)$ of simulated vimentin tetramers and ULF retrieved with a two components model fit.

obtained with a one component PCH model (equation 2.29) and a two components model (equation 2.32), for the three LRs tested. From the graph it is clear that for higher LRs, the two component $\mathrm{PCH}$ model can describe the data better. However, even for $\mathrm{LR}=10 \%$, the two component fit can retrieve the proper initial values. In Table 4.3, the parameters obtained with the two component model are summarized for the different labeling ratios. The fitted values are comparable with the calculated ones (Table 4.2). Nevertheless, for LR $=10 \%$, the errors of the fitting parameters are higher than to the ones for the other LRs. In fact, in principle at $\mathrm{LR}=10 \%$, even the one component model could describe the data $\left(\chi_{R}^{2} \approx 10^{-12}\right)$. The measured brightness retrieved with the single component model lies in between the brightness of the tetramer and the ULF. The average numbers in the observation volume are correctly retrieved in all three cases. However, as for the brightness, the fitting errors are higher in the lowest labeling ratio simulation. Observing the fitting parameters, on average, 
a.

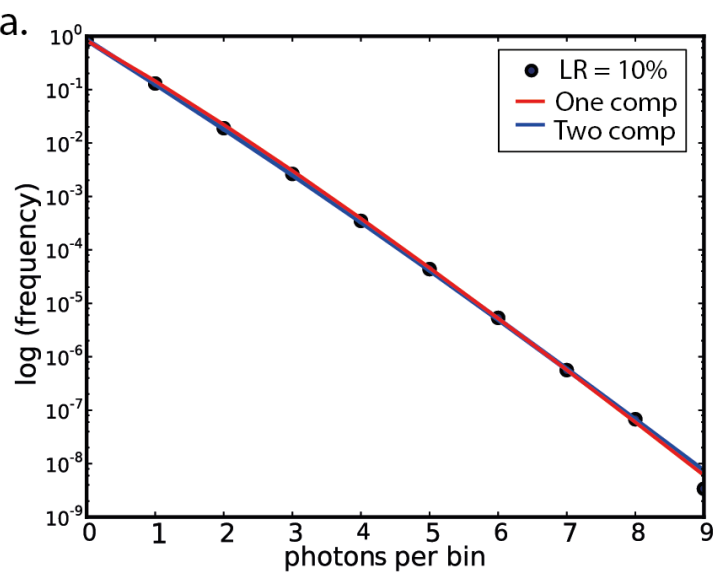

b.

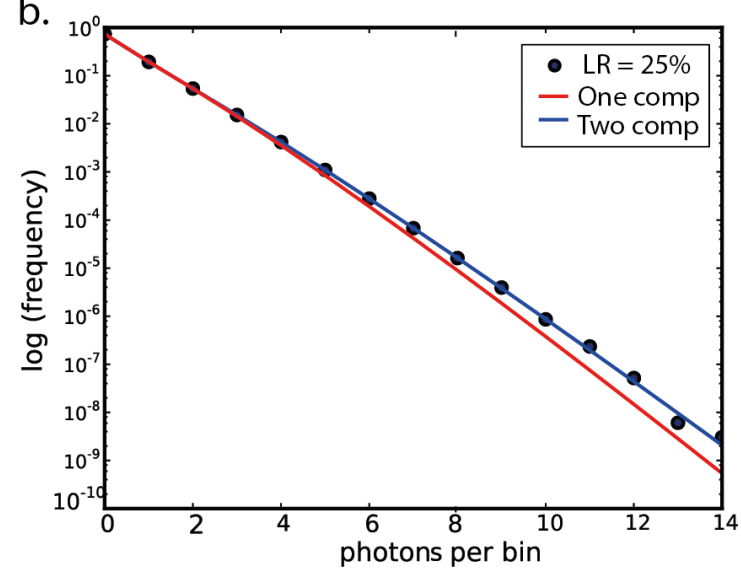

C.

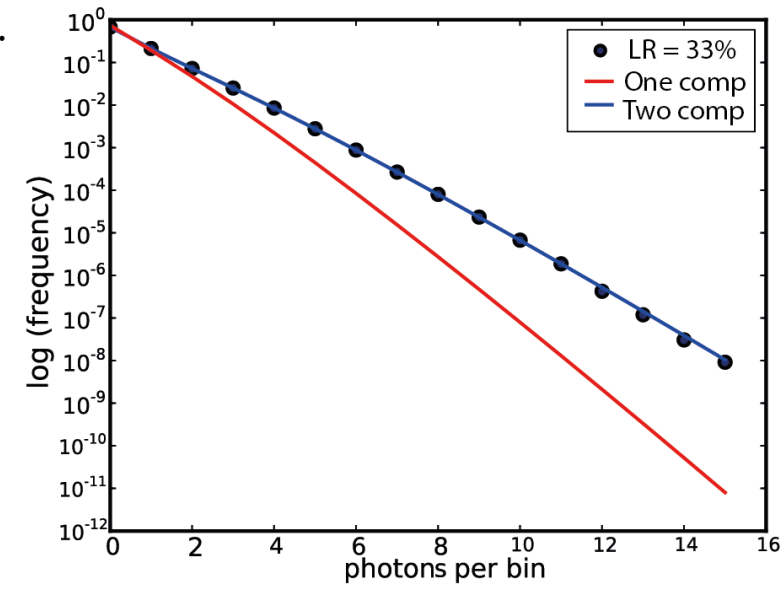

Figure 4.10: PCH data and fits for simulated vimentin assemblies, using the SimFCS software, at different assembling stages for different LRs. The simulated data in this figure represent the case of just tetramers and ULFs diffusing together. The PCH data are fitted with the selfwritten algorithm, with a one component model (red lines, equation 2.29) and a two component model (blue lines, equation 2.32) (a) At $\mathrm{LR}=10 \%$ it is very difficult to distinguish the two components, in fact the data can also be fitted with just one component fit. (b) As the labeling ratio increases a two components model describes better the data. (c) At $\mathrm{LR}=33 \%$ a single component fit is not in agreement with the data anymore. 


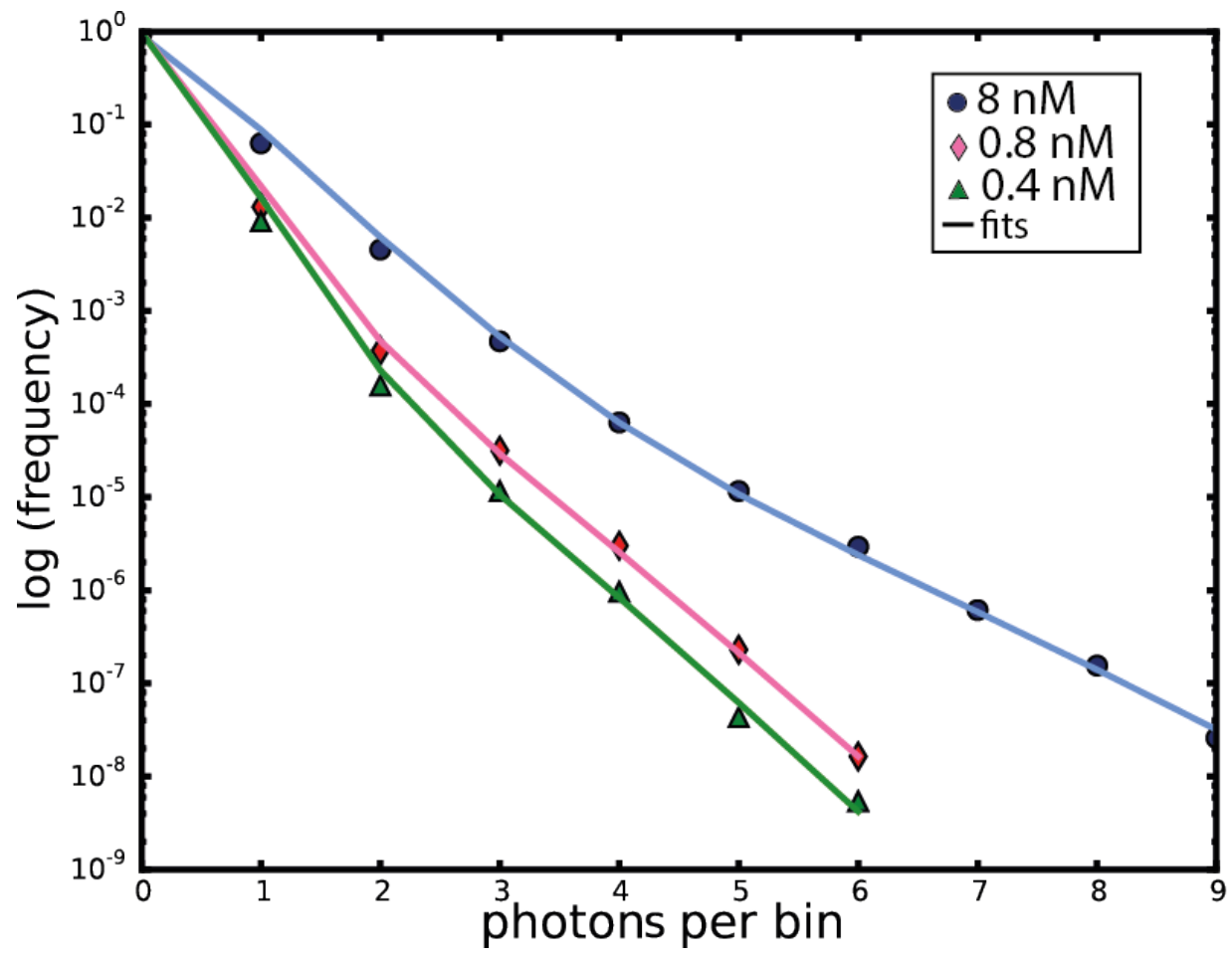

Figure 4.11: PCH of vimentin tetramers in bulk at three different concentrations. The symbols represent the experimental data and the lines the corresponding fits. The data are acquired for $300 \mathrm{~s}$ for each concentration.

the errors are lower than for the brighter component. That is probably due to the higher signal-to-noise ratio that the the brighter particles have. Since at a labeling ratio of $25 \%$ the two components can be correctly distinguished with low fit errors and good signal-to-noise ratio, vimentin samples have been mostly prepared with a labeling ratio between $25 \%$ and $33 \%$.

\section{Brightness of vimentin tetramers in bulk}

After the simulations described in the previous section, $\mathrm{PCH}$ measurements of vimentin tetramers can now be acquired with the setup. Vimentin tetramers are first measured in bulk at different concentrations to test if the $\mathrm{PCH}$ results can be reliably trusted. In Figure 4.11, $\mathrm{PCH}$ of vimentin tetramers in bulk at different concentrations $(8.0,0.8$ and $0.4 \mathrm{nM}$, which correspond at 0.01, 0.001, $0.0005 \mathrm{~g} / \mathrm{L}$ ) are shown. For the three samples, the brightness of a single vimentin molecule is constant with an average value of $106 \pm 2 \mathrm{kcps}$ while the average number of molecules in the observation volume decreases according to the concentrations, as summarized in Table 4.4 .

The PCH data can also be compared with the FCS curves. An example is shown in Figure 4.12, where the FCS and the PCH from vimentin at two labeling 
634.1. Studying protein interactions and labeling stoichiometry under flow

\begin{tabular}{ccc}
\hline sample & $B[\mathrm{kcps}]$ & $N$ \\
\hline \hline $8.0 \mathrm{nM}$ & $101 \pm 3$ & $0.22 \pm 0.01$ \\
\hline $0.8 \mathrm{nM}$ & $113 \pm 1$ & $0.023 \pm 0.004$ \\
\hline $0.4 \mathrm{nM}$ & $104 \pm 2$ & $0.0106 \pm 0.0004$ \\
\hline \hline
\end{tabular}

Table 4.4: Fit parameters retrieved with a single component fit of the PCH data shown in Figure 4.11. The brightness values, $B$, are comparable with each others at the different concentrations. While, the average numbers of molecules per volume of observation, $N$, are decreasing accordingly with the concentrations measured.

ratios (LRs), $22 \%$ and $36 \%$, and at two different concentrations are shown. The brightness $(B)$ obtained from the single component fits are in agreement with each other between same the labeling ratios (Table 4.5). Actually, the brightness values are similar between the two labeling ratios, since the average number of labels per vimentin tetramer is similar in the two cases, 1.4 label molecules in average for $\mathrm{LR}=22 \%$ and 1.6 for $\mathrm{LR}=36 \%$.

The average number of molecules in the observation volume $N$ obtained with the PCH fits scales with the concentrations, as shown in Table 4.5. The numbers of molecules are in agreement also with the autocorrelation data, where a diffusion coefficient comparable with the value for vimentin tetramers is retrieved in all the conditions $\left(\langle D\rangle=27 \pm 2 \mu \mathrm{m}^{2} / \mathrm{s}\right)$.

a.

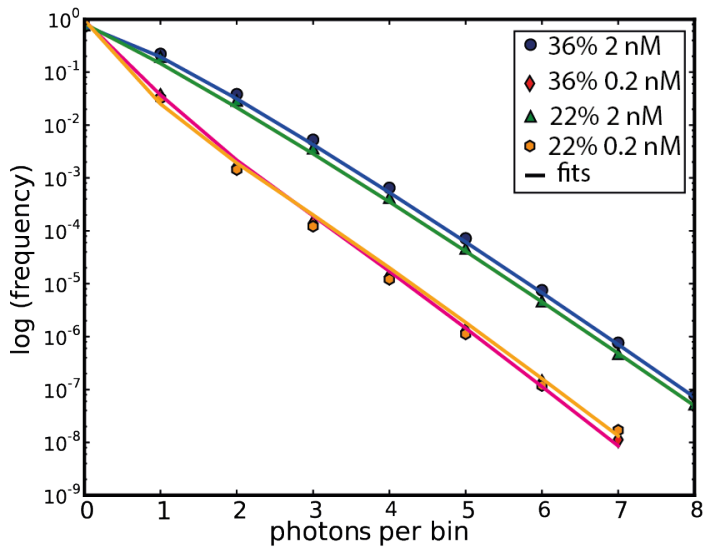

b.

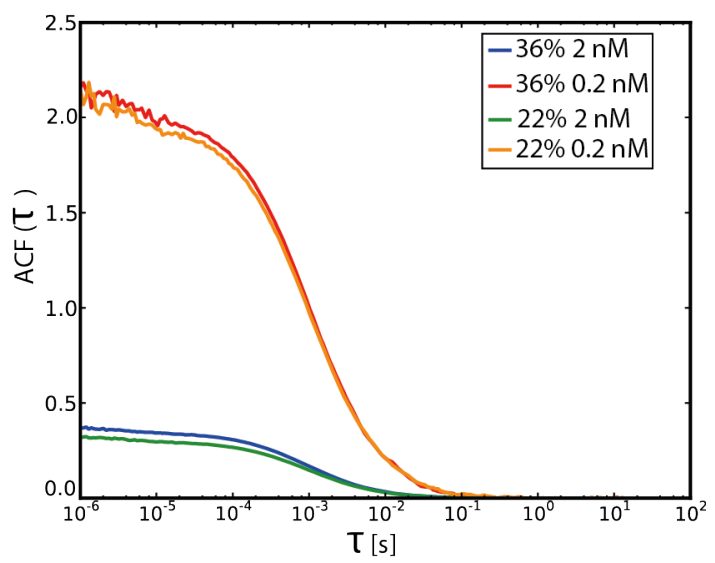

Figure 4.12: PCH (a) and FCS curves (b) of vimentin tetramers in bulk at different concentrations and LRs. The FCS curves are the average of 10 runs with $30 \mathrm{~s}$ of acquisition time each. The parameters obtained from the fits are listed in Table 4.5.

The brightness of vimentin tetramers in general might show some variability. This could be caused by small changes in the setup or by intrinsic protein variability during the labeling of vimentin molecules. In fact, the number of label per vimentin sub-unit is calculated using the binomial distribution (equation 4.1) which is an average value of the label distributions in the sub-units. In gen- 


\begin{tabular}{cccccc}
\hline LR [\%] & $C[\mathrm{nM}]$ & $B[\mathrm{kcps}]$ & $N_{P C H}$ & $N_{F C S}$ & $D\left[\mathrm{\mu m}^{2} / \mathrm{s}\right]$ \\
\hline \hline 22 & 2 & $96 \pm 3$ & $1.36 \pm 0.08$ & $1.201 \pm 0.008$ & $27 \pm 2$ \\
\hline 22 & 0.2 & $100 \pm 1$ & $0.14 \pm 0.02$ & $0.1739 \pm 0.0007$ & $29 \pm 2$ \\
\hline 36 & 2 & $104 \pm 2$ & $1.01 \pm 0.04$ & $1.04 \pm 0.01$ & $26 \pm 2$ \\
\hline 36 & 0.2 & $100 \pm 2$ & $0.14 \pm 0.04$ & $0.1736 \pm 0.0007$ & $29 \pm 1$ \\
\hline \hline
\end{tabular}

Table 4.5: Summary of the fitting parameters of curves shown in Figure 4.12. The data represent $\mathrm{PCH}$ and FCS measurements of vimentin tetramers in bulk of samples at two LRs with two different concentrations. The brightnesses measured are in agreement with each other and they are similar between the two labeling ratios since the average number of fluorophores per vimentin tetramer is similar in both cases. The values of $N$ obtained with the PCH analysis are in agreement with the values obtain from the FCS fits. In all four cases, a diffusion coefficient compatible with vimentin tetramers is measured with FCS.

eral, when the number of molecules, $N$, in the observation volume increases, for $N>3$, the PCH curves are more difficult to fit because the detector artifacts, as dead-time or afterpulsing, start playing an important role in the signal.

\subsubsection{Vimentin tetramers in flow}

Here, the measurements of vimentin tetramers in the two microfluidic devices, device $\mathrm{A}$ and device $\mathrm{B}$, are presented. In the first microfluidic device (device A), which is a single channel, $\mathrm{PCH}$ measurements of vimentin tetramers in flow are acquired to verify the independence of the $\mathrm{PCH}$ from the flow velocity. The second device (device B) is the five-inlet microfluidic step device, which is created to study the assembly of vimentin (described in Section 4.1.1).

\section{PCH of vimentin tetramers at different flow velocities}

In general, with higher flow rates the number of recorded fluorescent events increases. Figure 4.13 shows the time trace of the fluorescence counts for a sample containing diluted fluorospheres (FluoSpheres Carboxylate-Modified Microspheres $0.02 \mu \mathrm{m}$, Nile Red fluorescent (535/575), Thermo Fisher Scientific) with and without applied flow. The sample flowing with a velocity of $1 \mathrm{~mm} / \mathrm{s}$ has more events than the one without applied flow. Increasing the acquired events is useful when samples with low concentrations are measured, because it allows to decrease the acquisition times. It is known that FCS is affected by the flow velocity [27]: when the flow velocity is too high diffusion cannot be measured anymore, as explained in Section 2.1.2.

To verify that the photon count distribution is unchanged when convective flow is applied [53], vimentin tetramers are measured in a microfluidic device at different velocities. The microfluidic device used here has the simplest possible geometry. It is just a single channel with a width of $250 \mu \mathrm{m}$ and an height of $25 \mu \mathrm{m}$ (see Section 3.3.1). At this aspect ratio, the velocity in the device has a plug flow 


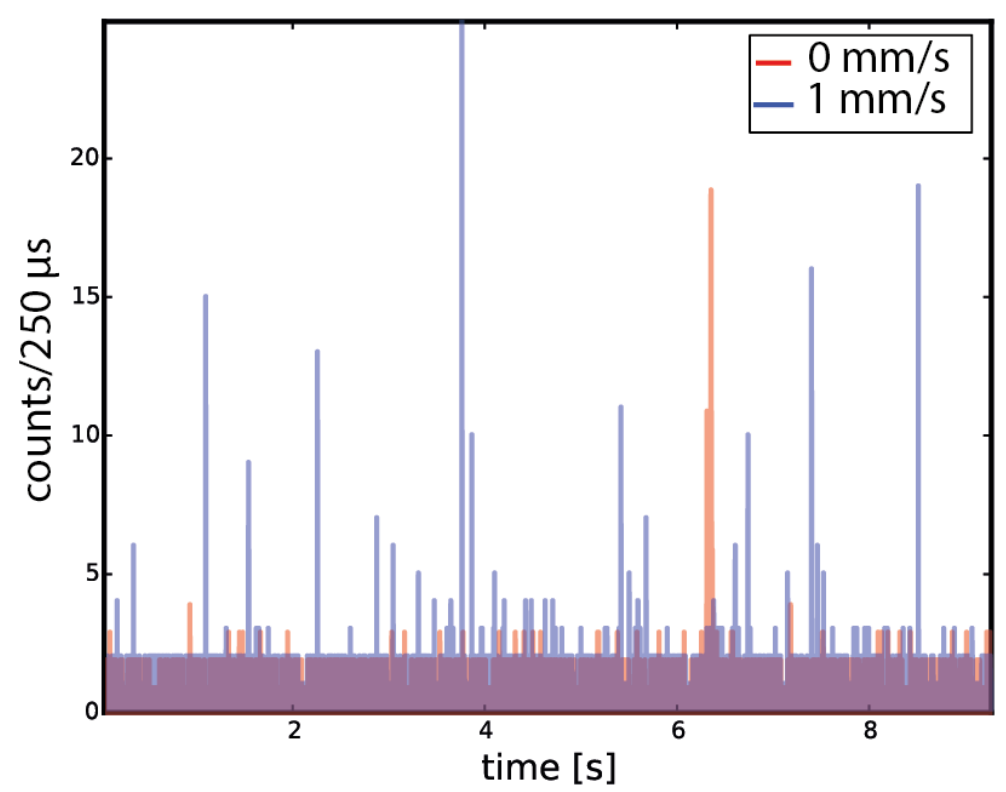

Figure 4.13: Fluorescence intensity trace over time for a sample of fluorospheres (20 nm, Nile Red fluorescent) diluted in water 1 to 2000, in a channel with and without flow. Note how the number of events increases when flow is applied (blue).

profile, as shown in Figure 4.14. Since the velocity is constant along the width of the channel, we can be sure that molecules will pass through the PSF with the same flow parameters. In this microfluidic device no changes in concentration (no dilution or assembly) or in brightness (the assembly is not initiated) are expected, thus the PCHs at different velocities should lay on top of each others, as long as undersampling is avoided.

Figure 4.15 a shows an example of $\mathrm{PCHs}$ measured at 4 different velocities $(2 \mathrm{~mm} / \mathrm{s}, 6 \mathrm{~mm} / \mathrm{s}, 10 \mathrm{~mm} / \mathrm{s}$ and $20 \mathrm{~mm} / \mathrm{s})$ for the same vimentin tetramers sample with a concentration of $2 \mathrm{nM}(0.002 \mathrm{~g} / \mathrm{L})$. To avoid undersampling, the sampling time has to be faster than the characteristic time scale of the fluorescence fluctuations. In this case, diffusion does not play a role anymore in the fluctuation time scale because the convective flow is too fast, in fact $\tau_{D}$ is $\approx 1020 \mu \mathrm{s}$ for vimentin tetramers and, with the velocities applied here, $\tau_{\mathrm{F}}$ (the flow time) varies between $150 \mu \mathrm{s}$ and $15 \mu \mathrm{s}$. The sampling time then has to be faster than $15 \mu \mathrm{s}$. Here $T=5 \mu$ is chosen as sampling time.

The four PCHs lay on top of each other confirming the model for PCH under flow. Moreover, the parameters obtained from the fits are in agreement with each other. In Table 4.6 the fitting parameters of the $\mathrm{PCH}$ shown in Figure 4.15, are reported. Both $N$ and $B$ are constant over the different velocities. The FCS curves at these velocities cannot be used to measure diffusion, but they can be 
a.

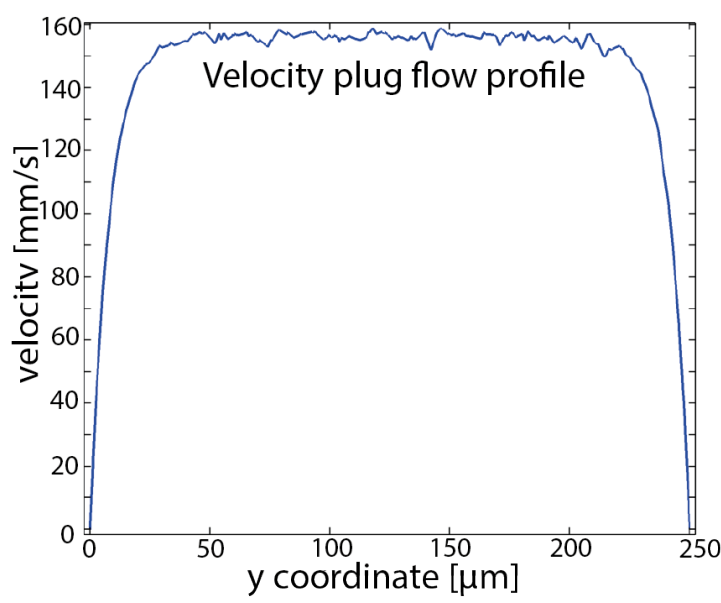

b.

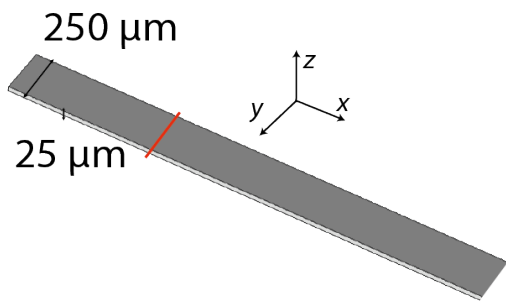

Figure 4.14: (a) Velocity plug flow profile of vimentin flowing in a microfluidic device of $250 \mathrm{\mu m}$ $\times 25 \mu \mathrm{m}$. The velocity has a constant value in the central section of the width of the channel. (b) Simulated channel, the red line marks the position of the line plot.

a.

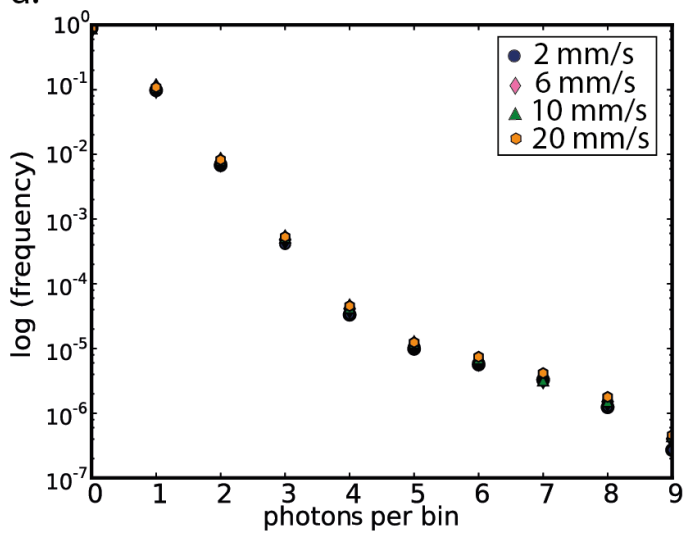

b.

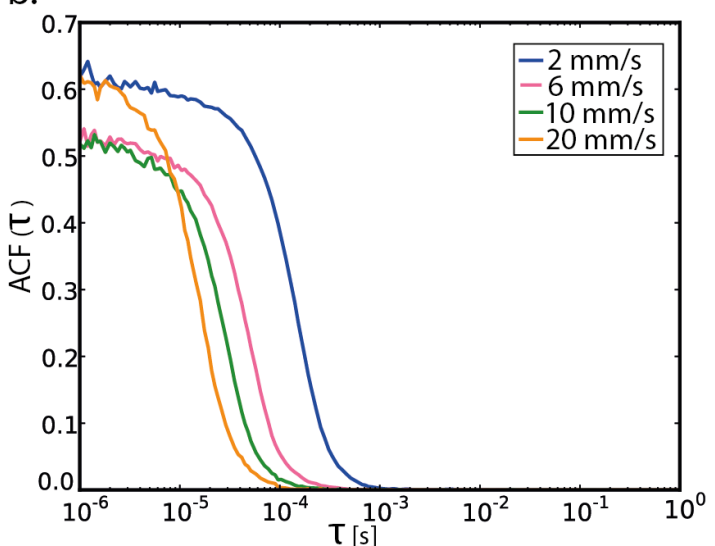

Figure 4.15: PCH and FCS curves of vimentin tetramers in flow at different velocities. (a) All PCHs are on top of each other confirming the independence of the $\mathrm{PCH}$ model from the flow velocity. (b) The FCS curves show the typical steepening caused by convective flow. The fluorescence intensities are acquired for $300 \mathrm{~s}$ for each PCH, while the FCS curves showed here are the average of 10 runs with $30 \mathrm{~s}$ of acquisition times each. 
674.1. Studying protein interactions and labeling stoichiometry under flow

\begin{tabular}{cccc}
\hline$v_{\text {input }}[\mathrm{mm} / \mathrm{s}]$ & $B[\mathrm{kcps}]$ & $N$ & $v_{F C S}[\mathrm{~mm} / \mathrm{s}]$ \\
\hline \hline 2 & $23.1 \pm 0.4$ & $0.73 \pm 0.01$ & $2.00 \pm 0.03$ \\
\hline 6 & $21.6 \pm 0.4$ & $0.71 \pm 0.01$ & $6.1 \pm 0.1$ \\
\hline 10 & $21.2 \pm 0.4$ & $0.83 \pm 0.02$ & $10.2 \pm 0.5$ \\
\hline 20 & $20.8 \pm 0.4$ & $0.921 \pm 0.002$ & $18 \pm 1$ \\
\hline \hline
\end{tabular}

Table 4.6: Parameters retrieved from the analysis of PCHs and FCS curves showed in Figure 4.15. Vimentin tetramers are measured in a single channel with FCS and PCH.

used to measure the flow velocity. The velocities calculated from the FCS curves faithfully recover the experimental conditions (Table 4.6). The amplitudes of the FCS curves have a small variability, this could be caused by variability at the single molecule level in the sample during the acquisition time. Nevertheless, the numbers of molecules in the observation volume calculated from the FCS analysis are in agreement with the $\mathrm{PCH}$ measurements.

\section{Vimentin tetramers in the step device measured with $\mathrm{PCH}$}

Vimentin tetramers are measured in the microfluidic device used also to study the assembly. In the previous section, the independence of the $\mathrm{PCH}$ from the flow velocity is proved, here we want to test the stability of vimentin tetramers in the step device. The measured brightness $B$ is expected to stay stable over the different positions of the device, and the average number of molecules per volume of observation $N$ is expected to decrease. In Section 4.1.1 the characterization of the microfluidic device is described. It is a five-inlet device with a channel width of $200 \mu \mathrm{m}$ for all channels, and a height of $100 \mu \mathrm{m}$ for the central inlet and $200 \mu \mathrm{m}$ for the remaining channels. Vimentin tetramers are delivered to the central channel with a concentration of $0.0025 \mathrm{~g} / \mathrm{L}(9 \mathrm{nM}$ for the vimentin batch used in this experiment) and a flow rate of $11 \mu \mathrm{L} / \mathrm{h}$. The vimentin concentration measured in $\mathrm{nM}$ is dependent on the labeling ratio of that particular vimentin batch, while the concentration in $\mathrm{g} / \mathrm{L}$ describes the overall protein concentration. Into the remaining inlets, $2 \mathrm{mM}$ of $\mathrm{PB}(\mathrm{pH} 7.5)$ are injected with a flow rate of $190 \mu \mathrm{L} / \mathrm{h}$ for the side inlets and $10 \mu \mathrm{L} / \mathrm{h}$ for the diagonal inlets. The PCH is measured at different positions along the center of the outlet channel, as sketched in Figure 4.2. In this case, the PCHs will not look identical to each other, because the protein concentration is changing through the device, as shown in the FEM simulations (Figure 4.7). However, the brightness should stay constant at the different positions measured because the assembling ions are not present in the buffer and thus, the protein remains in the tetramer form.

As predicted, the brightness is constant over the different positions in the microfluidic device, as shown in Figure 4.16a. In this experiment, every dot represents a $\mathrm{PCH}$ measurement with $500 \mathrm{~s}$ of acquisition time. The time on the $x$-axis is calculated from the velocity in the central channel obtained by the FEM 
a.

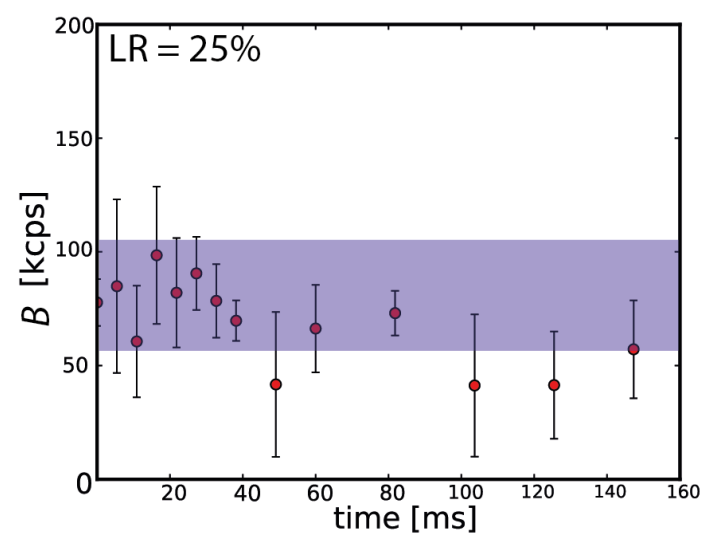

b.

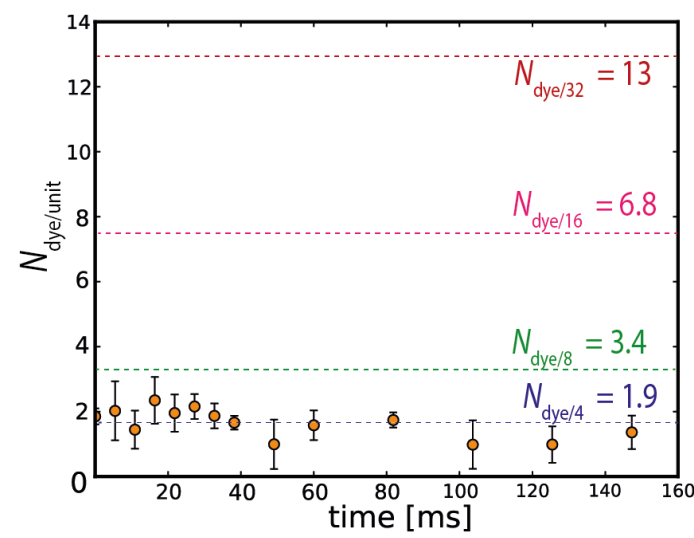

Figure 4.16: Brightness and number of Atto-532 per vimentin tetramer, obtained measuring vimentin tetramers with $\mathrm{PCH}$ in the five-inlet step device. Each dot corresponds to a $\mathrm{PCH}$ built after an acquisition time of $500 \mathrm{~s}$. The error bars correspond to the standard deviation of the fitting parameters. The time on the $x$-axis is calculated using the velocity values from the FEM simulations. (a) The brightness is constant along the outlet within one standard deviation (blue colored box, it is center on the measured average brightness). (b) The number of labels per vimentin molecule is calculated dividing the brightness of vimentin tetramers with the brightness of a single Atto-532 molecule measured in bulk. The number of labels per molecule shows no increase in time, confirming the presence of only vimentin tetramers. The values measured are in agreement with the expected number of labels per each vimentin assembly sub-unit calculated with the binomial distribution for vimentin tetramers (dotted lines).

simulation. The number of labels per vimentin sub-unit can be calculated once the brightness of a single molecule of Atto-532 is known. From bulk measurements, with the same acquisition conditions, the brightness for a molecule of Atto-532 is measured to be $B=54 \pm 20 \mathrm{kcps}$. In Figure $4.16 \mathrm{~b}$ the measured number of labels per molecule is shown over time. The values are similar to the expected number of label per vimentin tetramer calculated with equation 4.1. On average $1.6 \pm$ 0.4 labels per molecule are measured in the experiment, which is comparable to the expected value $\left(N_{d y e / 4}=1.9\right)$ calculated with the binomial distribution (equation 4.1). The brightness is actually constant in the different positions of the microfluidic device and, as expected, indeed corresponds, withing one standard deviation, to the vimentin tetramer value.

The average number of molecules per observation volume, $N$, is decreasing along the outlet channel, as predicted by the simulation (Figure 4.7). In Figure 4.17 the average number of molecules per observation volume, $N$, is plotted against the position in the channel and the assembling time. The origin for the bottom $x$-axis is in the central inlet. At $x=500 \mu \mathrm{m}$, the central inlet crosses the other channels. At the top $x$-axis the corresponding assembly time is shown. The 0 time point corresponds to the position in the channel where the $\mathrm{KCl}$ concentration is $10 \mathrm{mM}$ (in the simulations), as this concentration is the threshold for 


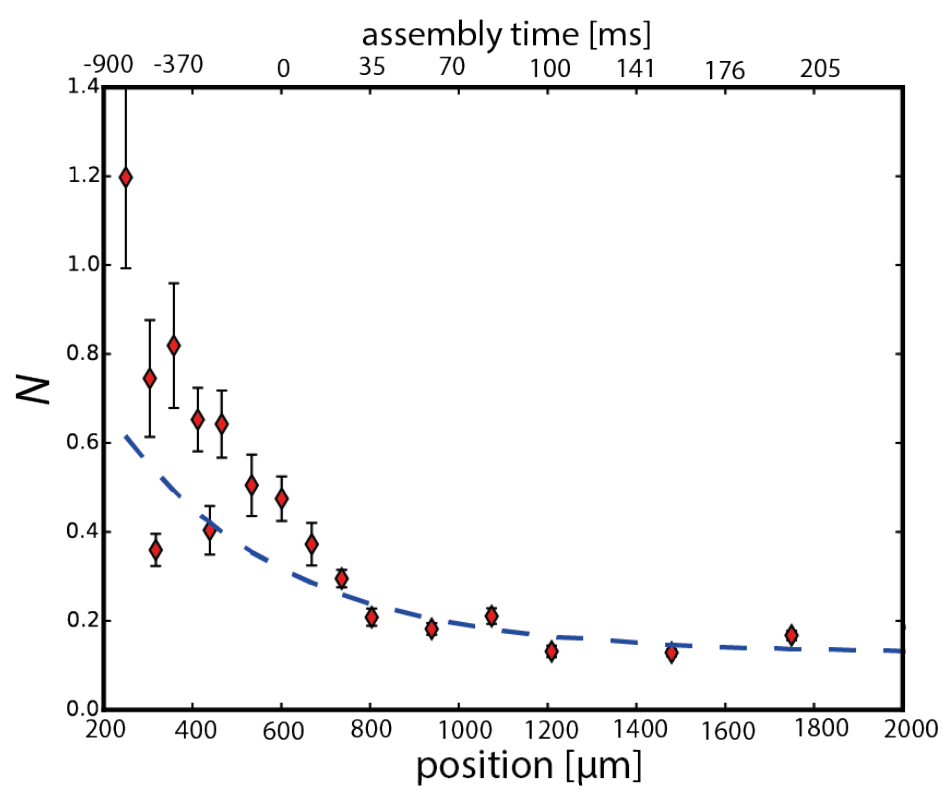

Figure 4.17: Number of molecules per observation volume for vimentin tetramers in the step device. The bottom $x$-axis represents the position of the measurement in the central channel of the device. The top $x$-axis shows, for the positions measured, the corresponding assembly time. The dotted line corresponds to an exponential decay fit used to qualitatively described the decreasing of $N$.

vimentin assembly [87]. The time axis is not spaced uniformly because the velocity is not constant along the outlet channel. The time points shown in the axis correspond to the measured position in the channel. The decrease of $N$ along the outlet channel is expected from the FEM simulations, however, the concentration of vimentin tetramers decreases faster than in the simulation. In fact, for the same positions in the device, the ratio of the initial value, $N_{0}$, and the last value, $N_{F}$, is $N_{0} / N_{F}=11.8$, in the experiment and $N_{0} / N_{F}=5$ in the simulation. In these experiments, where no ions are added in solution to start the assembly, the decrease of vimentin tetramers along the outlet channel should have been caused only by the dilution of the protein solution with the PB buffers from the other four inlets. As consequence, the measured ratio $N_{0} / N_{F}$ should have been the same to the simulated one. However, it seems that when vimentin tetramers are measured in the step device, the concentration decreases faster than expected. The decrease is also qualitatively followed fitting the curves of $N$ over time using an exponential decay function, described by:

$$
N(t)=N_{0}\left(e^{-t / \tau_{N}}\right)+C
$$

where $\tau_{N}$ is the characteristic decay time for the fitted curve. For the case of vimentin tetramers in the microfluidic device (Figure 4.17) the characteristic time decay has a value of $\tau_{N}=46 \pm 10 \mathrm{~ms}$, while in the simulation a decay time of 


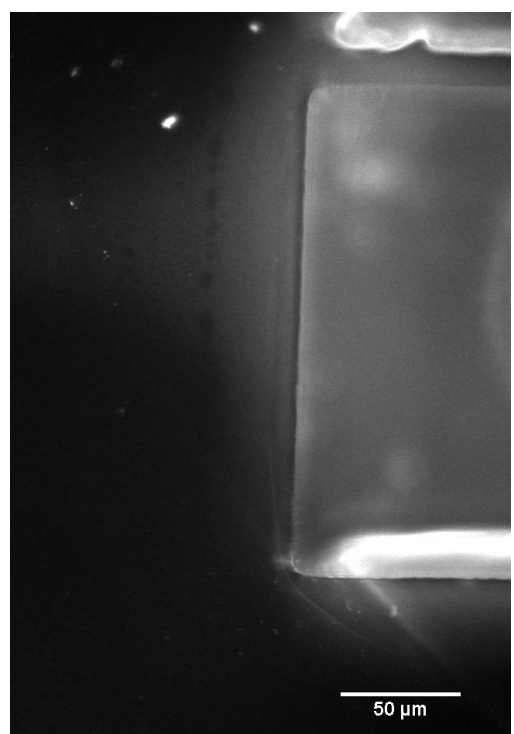

Figure 4.18: Epi-fluorescence image of the NOA glue step inside the microfluidic device during an experiment with vimentin tetramers in flow. Vimentin tetramers are adsorbed to the NOA step.

$\tau_{N}=70 \pm 2 \mathrm{~ms}$ is obtained. The exponential fits confirm that the decrease of $N$ in the microfluidic device is faster than in the FEM simulations.

A possible explanation of this not expected fast decrease of $N$ in the device could be related to adsorption of vimentin tetramers by some components of the microfluidic device, such as the glue used to create the step in the central inlet. In fact, with the step device protein adsorption is prevented in the central mixing region, but not before that. In Figure 4.18 an epi-fluorescence image of the microfluidic device during one experiment with vimentin tetramer is shown. In particular, vimentin tetramers are adsorbs to the UV-curable step created on the glass slide. It appears that a Some vimentin molecules are also adsorbed by the PDMS side walls of the central channel (next to the step). It could be that indeed two different "types" of concentration decay are taking place in the device. At first, the protein concentration decreases since vimentin molecules are adsorbed on the step in the central inlet, then the protein concentration decreases because of the dilution of the sample by the fluids from the other inlets. Possibly, two regions with different decay times, a first fast decay related to the protein adsorption and a slower decay caused by dilution, might be distinguishable in the graph of $N$ at different positions in the device, however we do not have enough data points to distinguish properly the two behaviours.

The data just presented are analyzed using a model for two components for $\mathrm{PCH}$. The second component measured has a constant brightness along the channel, as shown in Figure 4.19a. In every measurement, the second component is brighter than the expected brightness for a ULF (Figure 4.19a). Since, here, 
a.

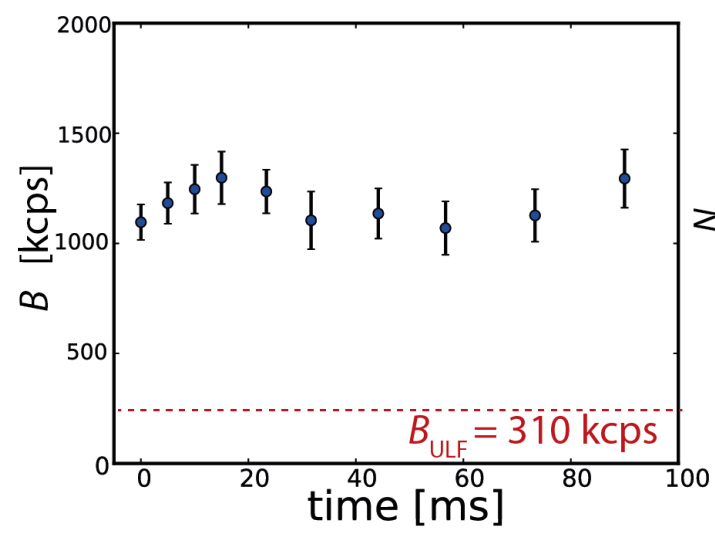

b.

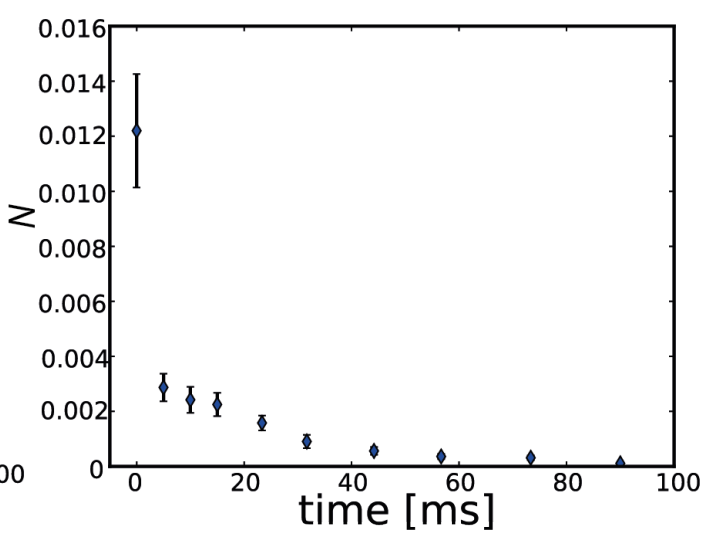

Figure 4.19: Brightness $B$ and average number of molecules per observation volume $N$ for the second component found during the analysis of the PCHs for vimentin tetramers in flow. (a) The brightness is constant over time. The average brightness value of the second component is higher than the values measured, and calculated, for vimentin assemblies. (b) The number of molecules per volume is decreasing along the channel.

$\mathrm{KCl}$ ions are not added into the buffer solution, assemblies of vimentin are not expected, thus the measured second component, probably, is not a result of vimentin ordered assembly. However, in Lopez et al. [87], aggregates of vimentin were found during scattering measurements even before the assembly was initiated. Random aggregates of protein could be a cause of the second component.

The values found for the second component are consistent with other measurements of vimentin in the microfluidic devices, even during the assembly experiments. Nevertheless, since the brightness of this second component is very different from the brightness of vimentin assemblies, we can still clearly distinguish vimentin sub-units in our measurements. The second component is not present in $\mathrm{PCH}$ measurements of dyes, as rhodamine 6G or Alexa-532 in flow or in vimentin samples measured in bulk. By now, no clear explanation has been found to explain the second component.

\subsubsection{Measurements of vimentin lateral assembly under flow}

Vimentin and assembly buffer are mixed inside a microfluidic device that allows us to access the temporal evolution of the assembly process. The five-inlet microfluidic device is employed with a constriction in the central inlet, as already described in the previous sections. Vimentin tetramers with a concentration of $0.0025 \mathrm{~g} / \mathrm{L}$ are loaded into the central channel with a flow rate of $11 \mu \mathrm{L} / \mathrm{h}$. In the side inlets, $100 \mathrm{mM}$ of $\mathrm{KCl}$ in $2 \mathrm{mM} \mathrm{PB} \mathrm{(pH} \mathrm{7.5)} \mathrm{are} \mathrm{flushed} \mathrm{to} \mathrm{start} \mathrm{the} \mathrm{assembly}$ with a flow rate of $190 \mu \mathrm{L} / \mathrm{h}$, and $2 \mathrm{mM} \mathrm{PB}$ buffer $(\mathrm{pH} 7.5)$ is used with a flow rate of $10 \mu \mathrm{L} / \mathrm{h}$ for the diagonal inlets. The measured brightness, $B$, number of labels per sub-unit, $N_{\text {dye/unit }}$, and number of molecules in the observation volume, 
$N$, correspond to the average values for all molecules in the observation volume during one sampling time, which is set to $5 \mu \mathrm{s}$.

$\mathrm{PCH}$ measurements are employed to measure the labeling stoichiometry of each vimentin sub-unit during the assembly, e.g. tetramers, octamers or ULFs. For every PCH measurement the fluorescence intensity is acquired for $800 \mathrm{~s}$. Since during the assembly vimentin molecules associate, we can expect the brightness to increase over time. In the microfluidic device employed, the elongation of vimentin cannot be measured as this assembly step occurs in the time scale of minutes and the maximum time point measurable with this device is around $4 \mathrm{~s}$. However, we are able to precisely measure the lateral assembly of vimentin, up to the formation of ULF. As the brightness, $B$, increases the average number of molecule per observation volume, $N$, decreases over time. While the increase in brightness is caused only by the assembly, the decrease of $N$ is caused partly by the assembly and partly by the dilution of the central stream by the injected buffer from the other inlets. The changing brightness over time is exploited to calculate the number of labels per vimentin sub-unit during the assembly. The measured label stoichiometry is then compared with the predicted label stoichiometry, calculated with the binomial distribution (equation 4.1). An exponential fit is employed to characterize the curves of $B$ over time and to quantify the time scale of the ULF assembly.

In the following part, some example of vimentin assembling experiments will be described. Figure 4.20 shows an example of vimentin assembling in the fiveinlet step microfluidic device. The change in brightness and in label stoichiometry over time are shown. In this case, the labeling ratio of vimentin is $\mathrm{LR}=28 \%$. The brightness is increasing over time until a plateau value, which corresponds to the brightness of ULFs, as shown in Figure 4.20 $\mathrm{a}$. The increase in measured brightness corresponds to the lateral assembly of vimentin tetramer forming octamer, hexadecamers and ULFs. Knowing the brightness value for a single molecule of Atto-532, we can calculate the stoichiometry of the vimentin sub-units during the early time points of assembly. The number of labels per vimentin sub-unit increases in time, as the brightness, up to a plateau which corresponds to the value for ULFs (Figure $4.20 \mathrm{~b}$ ). At this labeling ratio, on average, 1.5 fluorophores are expected for each tetramer (value calculated from equation 4.1). In the experiment, a value of $2 \pm 1$ fluorophores is found at $t=0 \mathrm{~s}$, when the assembly has just started.

The time evolution of vimentin assembly can be qualitatively followed just looking at Figure $4.20 \mathrm{~b}$. The data suggest that octamers are formed in the first few milliseconds after the assembly and after $50 \mathrm{~ms}$ hexadecamers are formed. ULFs are already produced after $100 \mathrm{~ms}$ from the starting of the assembly. The time needed to form ULFs starting from tetramers is quantified with an exponential fit, from which a formation time of $45 \pm 10 \mathrm{~ms}$ is obtained in this case. However, at this labeling ratio, the measured number of labels per vimentin sub-unit at the plateau is lower than the value predicted for ULFs using equation 4.1. In 


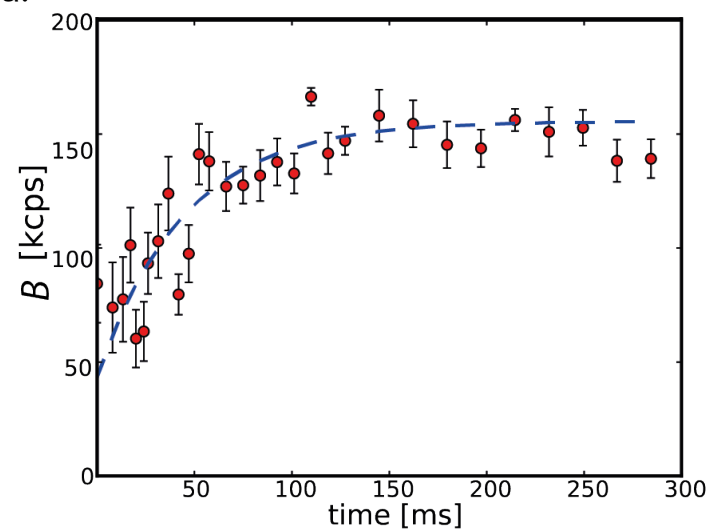

b.

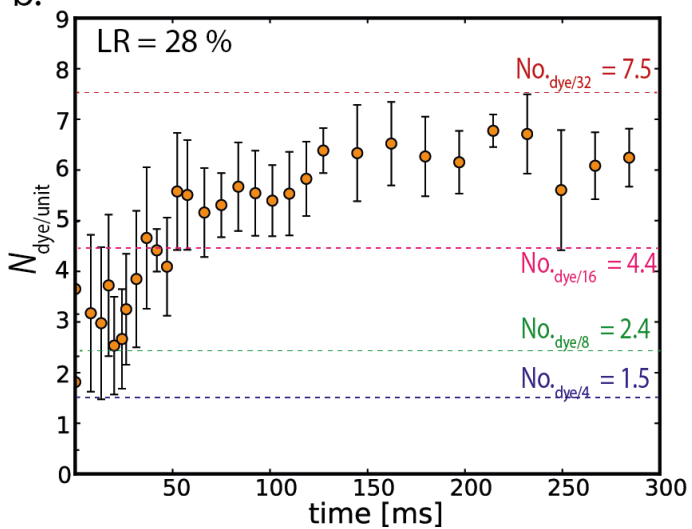

Figure 4.20: Example of brightness and number of label per sub-unit over time for an experiment of vimentin assembling in the five-inlet microfluidic device. In this case the labeling ratio is $28 \%$. (a) The brightness increases over time. The data points are fitted with an exponential growth function. (b) The number of labels per vimentin sub-unit is calculated dividing the brightness at each time point for the brightness of a single molecule of Atto-532 measured at the same experimental condition. The values for the number of label per sub-unit reach a plateau, which corresponds to ULFs. However, the measured values for the number of labels per ULF are lower than the predicted value (red dotted line). Possibly, the high labeling density induces self-quenching of the dye that might decreases the measured brightness for the ULF.

fact, the measured number of labels per ULF is, on average, $6.3 \pm 0.3$ while the theoretical value is 7.5. The measured difference reflects an effective decrease in the measured brightness of the labels, which is possibly caused by self-quenching of the fluorophores attached to the ULF. Self-quenching effects are dependent on the dye molecule itself and on the environment. In this case, in average, 3 to 4 dye molecules are attached to the tails of vimentin monomers in each side of a single ULF, which has a diameter of $17 \mathrm{~nm}$. Thus, on average, the dyes are separated from each other by a distance between 4 and $6 \mathrm{~nm}$, small enough to see quenching effects.

This explanation is supported by a measurements with vimentin at a lower labeling ratio (12\%), as shown in Figure 4.21. In this case, the measured number of labels per vimentin sub-units corresponds with the predicted value $4.21 \mathrm{~b})$.

In Figure 4.21, the brightness and the label stoichiometry are shown during the assembling of vimentin. The brightness increases rapidly, as before, and after $\approx 100 \mathrm{~ms}$ it reaches a plateau value. In particular a ULF formation time of $26 \pm$ $5 \mathrm{~ms}$ is here obtained with the exponential fit. This experiment shows that even if the labeling ratio is not as high as in other experiment, it is still possible to measure the average increase of $B$ over time. The number of labels per vimentin sub-unit measured, shown in Figure $4.21 \mathrm{~b}$, increases over time, starting from a value of $1.2 \pm 0.2$, which corresponds to the predicted value for tetramers $\left(N_{o}\right.$.dye $/ 4$ $=1.2$ ) for tetramers, up to a value of $4.5 \pm 0.5$ comparable to the predicted value 
a.

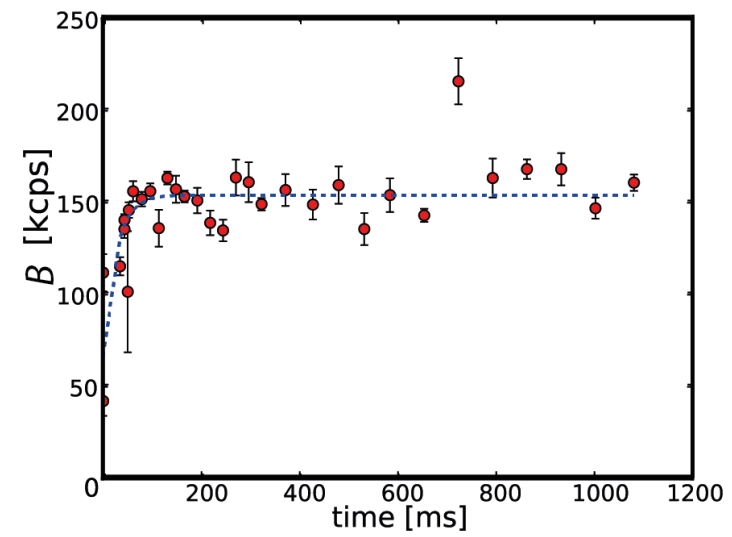

b.

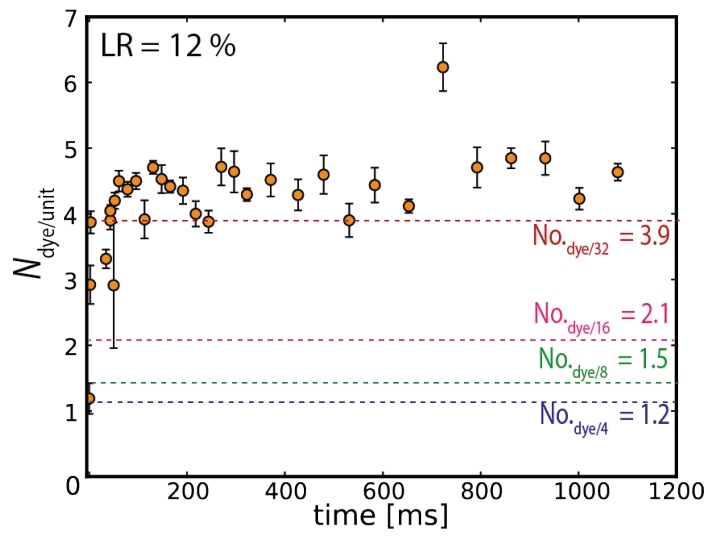

Figure 4.21: Example of brightness and label stoichiometry over time for vimentin assembling in a microfluidic device with low labeling ratio $(\mathrm{LR}=12 \%)$. (a) The brightness increases quickly, within $100 \mathrm{~ms}$ from the start of the assembly the brightness reaches a plateau value. The data are fitted with an exponential growth function to quantify the ULF formation time. (b) The number of label per vimentin sub-unit is calculated dividing the measured brightness with the brightness for a single Atto-532 molecule obtained at the same experimental conditions. The starting value corresponds to vimentin tetramers and the plateau value corresponds to ULF molecules. The number of labels predicted with the binomial distribution for each vimentin assemblies is depicted with dotted lines.

for ULFs $\left(N_{o \cdot d y e / 32}=4\right)$. After the formation of the ULFs, the sub-units start to assemble longitudinally forming filaments. However, higher orders of assembled vimentin are not visible in this experiment because they form at longer time scale (minutes) [87] and with this device the maximum time point reachable is $\approx 4$ s. As consequence, the plateau value reached by the brightness corresponds to the ULF formation step within the measurements errors. The first time point of the brightness measured is in agreement with the bulk measurement of the same vimentin sample before the assembly: the brightness measured in bulk for the same vimentin tetramers is $40 \pm 5 \mathrm{kcps}$, while in the assembly experiment, at $t$ $=0 \mathrm{~s}, B=41 \pm 8 \mathrm{kcps}$.

Until now, only the positive time points of assembly have been showed, in Figure 4.22 the brightness of vimentin from "negative" assembly time points is shown. The brightness is constant before the origin, confirming that the assembly is not triggered if the $\mathrm{KCl}$ has not reached a concentration of $10 \mathrm{mM}$, which is the position in the channel considered as the origin. The average brightness value measured before the origin, $B=60 \pm 6 \mathrm{kcps}$, is comparable with the brightness of the same vimentin tetrameric sample measured in bulk at the same condition, where $B$ is $54 \pm 5 \mathrm{kcps}$. After $\mathrm{KCl}$ has reach the threshold value of $10 \mathrm{mM}(t=0)$, vimentin molecules start to assemble, thus the increase of the brightness. In this case, as for the first example (Figure 4.20), the predicted brightness of ULFs (calculated with the binomial equation 4.1) is higher than 


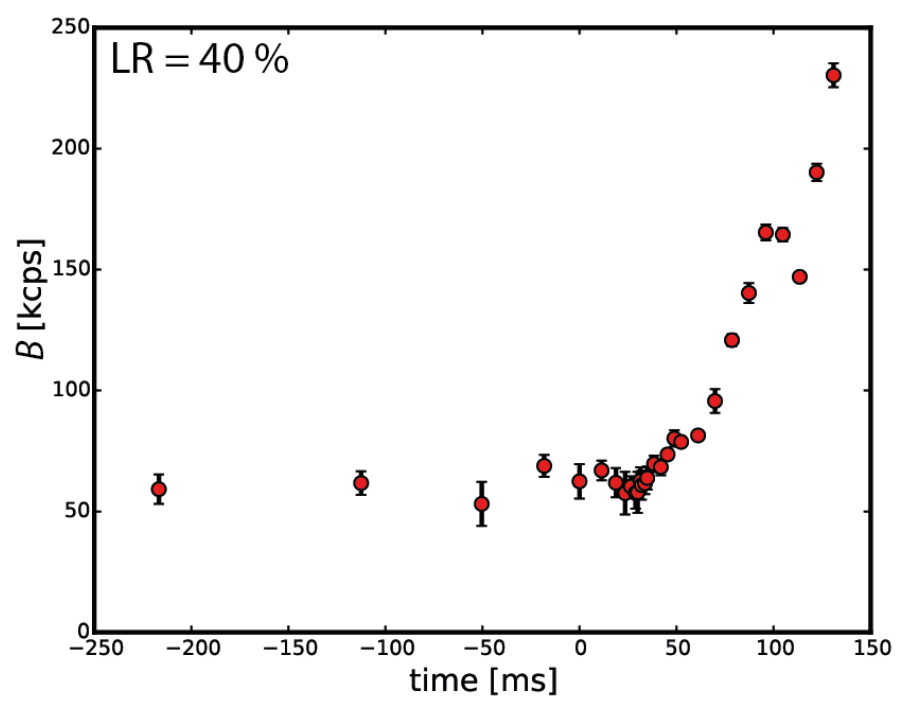

Figure 4.22: Brightness over time for a sample of assembling vimentin with a labeling ratio of 40 $\%$. At negative time points, the brightness is constant in time with a value which corresponds to vimentin tetramers. As soon as the assembly starts $(\mathrm{KCl}$ reaches the threshold value of 10 $\mathrm{mM}$ ), the measured brightness increases. The brightness does not reach a plateau even after $\approx 100 \mathrm{~ms}$ of assembly. Possibly, the high density of dye molecules slows down the assembly reaction.

the brightness measured with $\mathrm{PCH}$. At the labeling ratio of this vimentin sample (40\%) one ULF should have a brightness of $340 \mathrm{kcps}$, however here, after 130 ms of assembly, the measured brightness has a value of $240 \mathrm{kcps}$. The lower measured brightness can be possibly explained with self-quenching of the labeled fluorophores, caused by the high density of fluorophore per each ULF. However, since in this experiment the brightness has not reached a plateau, it is difficult to provide a clear explanation. In particular here, not only the brightness is lower than expected (for ULF) but it increases also slower than in the other experiments. The high labeling ratio might have influenced also the dynamic of the assembly, which is slowed down perhaps by the high fluorophore density for each vimentin sub-unit.

As the brightness increases, the average number of fluorescent molecules in the observation volume, $N$, decreases. In Figure 4.23, $N$ is reported versus the assembly time for two examples discussed previously, the one of vimentin tetramers at $\mathrm{LR}=12 \%$ (Figure $4.23 \mathrm{a}$ ) and the one with $\mathrm{LR}=28 \%$ (Figure $4.23 \mathrm{~b}$ ). The decrease of $N$ over time cannot be compared directly with the simulations, since with PCH only the fluorescent molecules are detected and in our experiments not all the molecules are labeled.

The behavior of $N$ over time is consistent in the different assembly experiments. In Figure $4.24 N$ over time is shown for two different experiments. In 
a.

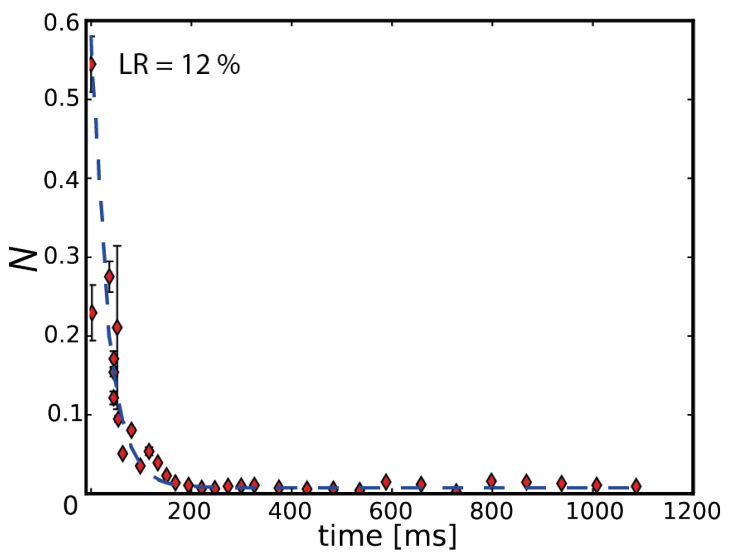

b.

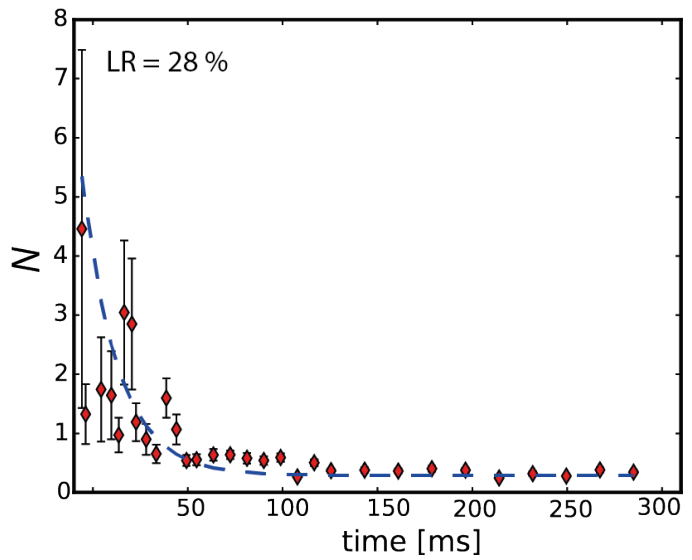

Figure 4.23: Average number of fluorescent particles per observation volume $N$ for two vimentin assembly experiments discussed previously. (a) $N$ over time for the experiment where vimentin is labeled at $12 \%$. (b) $N$ over time for the experiment where vimentin is labeled at $28 \%$. The average number of molecules in the observation volume is decreasing over time in both cases, as expected. The decrease of $N$ along the outlet channel is partially caused by the dilution of the vimentin sample and partially caused by the assembly of vimentin sub-units. The dotted blue lines correspond to exponential decay fitting curves.

both cases, the values are normalized for the maximum value of $N$. The overlap of the two curves confirms that, at the same experimental conditions, the lateral assembly has the same temporal evolution. Moreover, the time constants obtained fitting the curves with equation 4.2 are very similar to each other, confirming the reproducibility of our experiments when vimentin is measured at the same labeling ratio. The average number of particles, $N$, in the two datasets shown in Figure 4.23 are decaying with similar characteristic time, $\tau_{N}=33 \pm 2$ $\mathrm{ms}$ for the sample at $\mathrm{LR}=12 \%$ and $\tau_{N}=29 \pm 7 \mathrm{~ms}$ for the sample at $\mathrm{LR}=$ $28 \%$, confirming indeed the reproducibility of our experiments even for vimentin samples at different labeling ratios. When assembly ions are not added into the buffer solution, the decay time for $N$ is on average $44 \pm 13 \mathrm{~ms}$, which is slower than what measured when vimentin molecules are assembling.

The solution coming out from outlet of the microfluidic device, i.e. the assembled vimentin, has been measured directly after with FCS in bulk. In Figure 4.25, the $\mathrm{PCH}$ fits and the average FCS curves of before and after the assembly microfluidic device are shown. Before the assembly in the microfluidic device, vimentin tetramers are measured with FCS and PCH in bulk. With an observation volume of $330 \mathrm{~nm}$, a diffusion coefficient of $D=25.1 \pm 0.3 \mathrm{\mu m}^{2} / \mathrm{s}$ is obtained with FCS. This value corresponds to the predicted diffusion coefficient for vimentin tetramers, calculated using Stokes-Einstein equation 2.16, $D=22 \mathrm{\mu m}^{2} / \mathrm{s}$. After the device, two diffusing components are found in solution. The first one, $D_{1}=23.4 \pm 0.1 \mu \mathrm{m}^{2} / \mathrm{s}$, is compatible with vimentin tetramers, and the second 


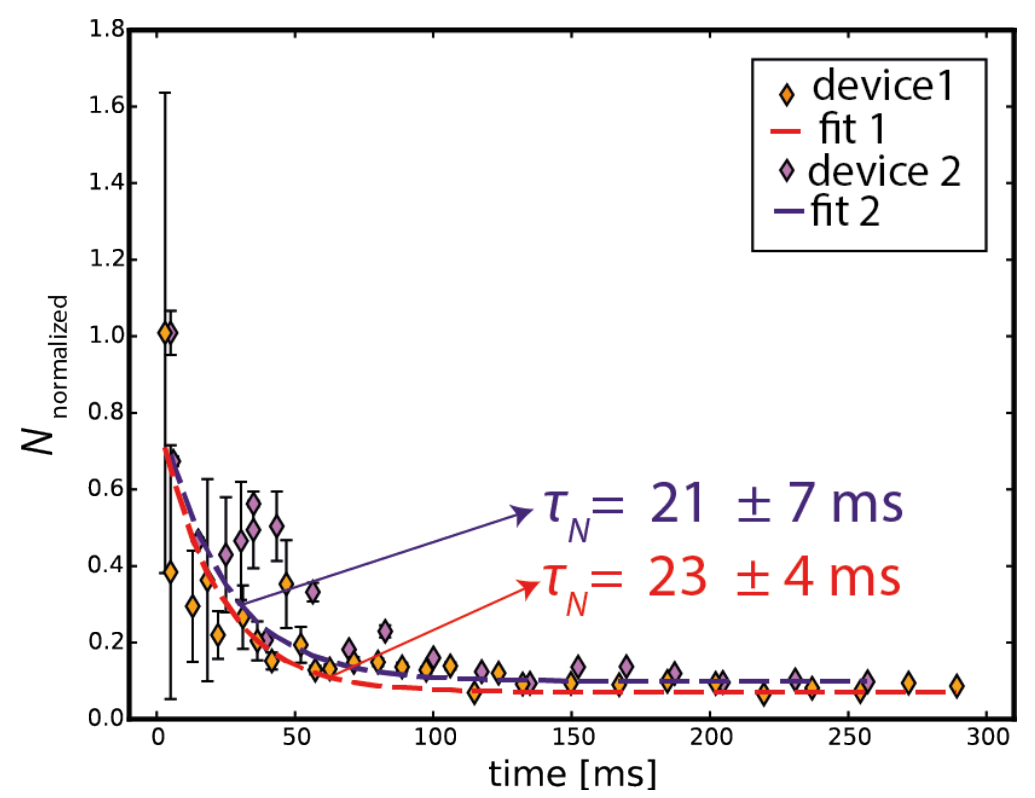

Figure 4.24: Normalized average number of molecule per observation volume over time for two independent experiments. The curves from the two experiments lie on top of each other, demonstrating the reproducibility of our method. The dotted lines represent the exponential decay (equation 4.2 fits. The two curves decay with similar time constants.

a.

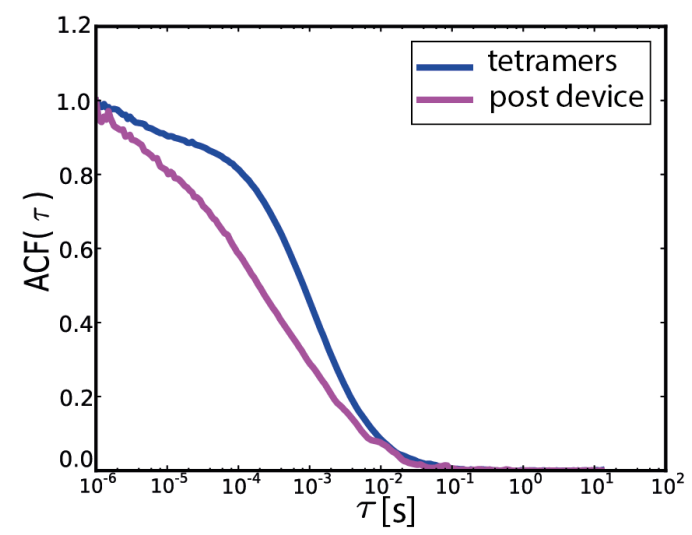

b.

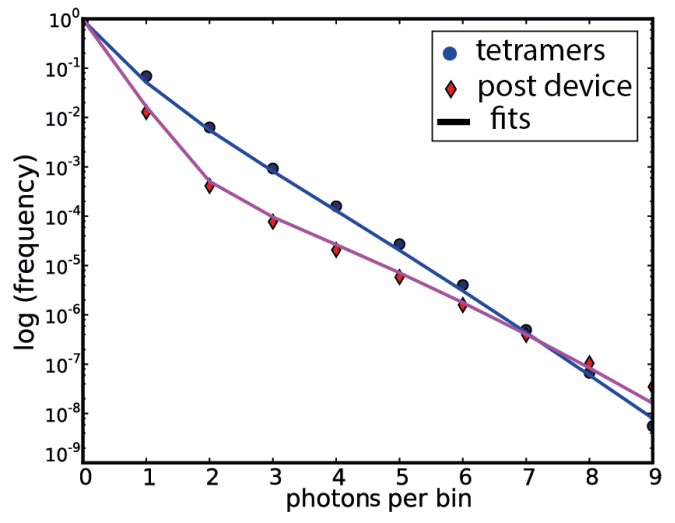

Figure 4.25: (a) FCS curves and (b) PCH of vimentin before and after the microfluidic device. The FCS curves shown are the normalized average of 15 runs, each of them was acquired for $30 \mathrm{~s}$. The fluorescence intensity was acquired for $900 \mathrm{~s}$ to build each PCH. 
a.

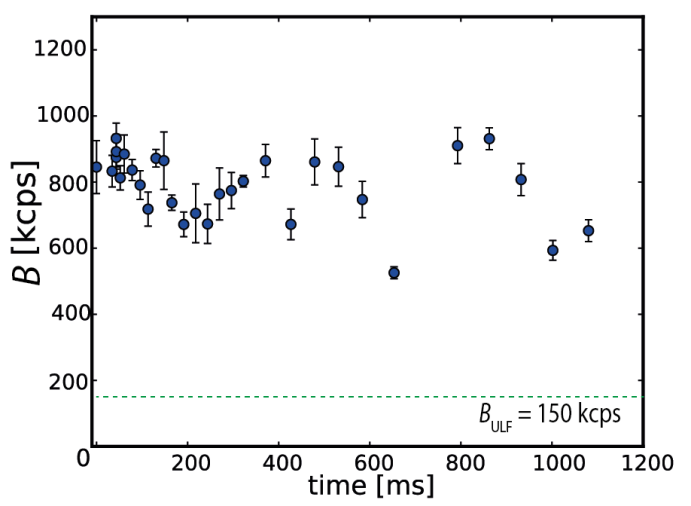

b.

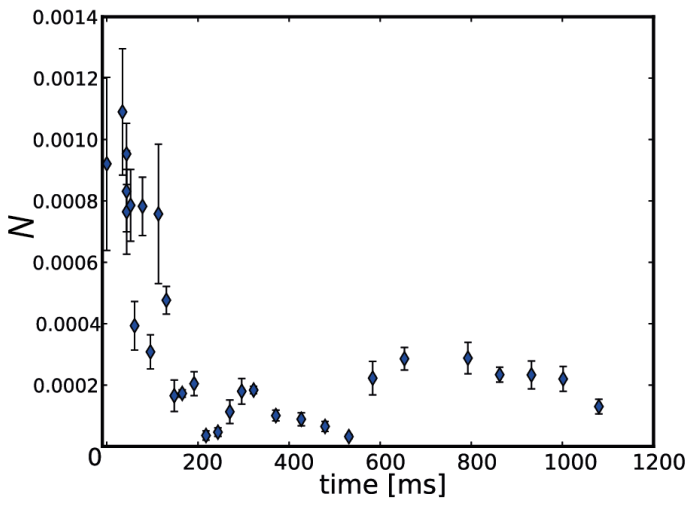

Figure 4.26: Brightness and average number of molecules per observation volume over time for the second fitting component of the low labeling ratio measurement.

one $D_{2}=7.5 \pm 2 \mu \mathrm{m}^{2} / \mathrm{s}$, fits with a larger assembly. The diffusion coefficient for ULFs can be estimated with a value of $D=10 \mathrm{\mu m}^{2} / \mathrm{s}$ from an equation described in [131], which describes the model of $D$ for a cylinder.

$$
D=\frac{k_{B} T}{3 \pi \eta L}\left(\log (L / d)+0.6 d / L-0.1 d^{2} / L^{2}+0.3\right)
$$

where $L$ is the length of the cylinder and $d$ the diameter, which for ULF are $60 \mathrm{~nm}$ and $17 \mathrm{~nm}$ respectively. Thus, the estimated value is in agreement with the measurement. PCH analysis confirms the presence of ULFs in the assembled solution. A brightness of $65 \pm 20 \mathrm{kcps}$ is measured in the sample containing only tetramers, which is similar to previous measurements on vimentin tetramers. By contrast, in the sample from the outlet of the microfluidic device, two brightness components are measured. One dimmer component of $B=68 \pm 7 \mathrm{kcps}$, reconcilable with vimentin tetramers, and one brighter component of $B=242 \pm 5 \mathrm{kcps}$, consistent with previous measurements of ULF molecules.

As for vimentin tetramers in the step device, a second brightness component is found also here. Figure 4.26, shows the brightness and the average number of particles per observation volume $N$ for the second brightness component obtained in the sample of vimentin at $\mathrm{LR}=12 \%$. The brightness of the second component is not varying over time, as in the experiment of vimentin tetramers under flow (Figure 4.19). Its average value is higher than the brightness value expected and measured for ULFs. The number of molecules per observation volume, for this component is also slightly decreasing over time and the maximum value is found at the beginning of the channel, close to the NOA glue step. Since this bright component is found when vimentin is measured in microfluidic device (even without assembly buffer) and not when other samples are measured in flow, as rhodamine $6 \mathrm{G}$ or Atto-532, it may be caused by unsystematic aggregation of the 
a.

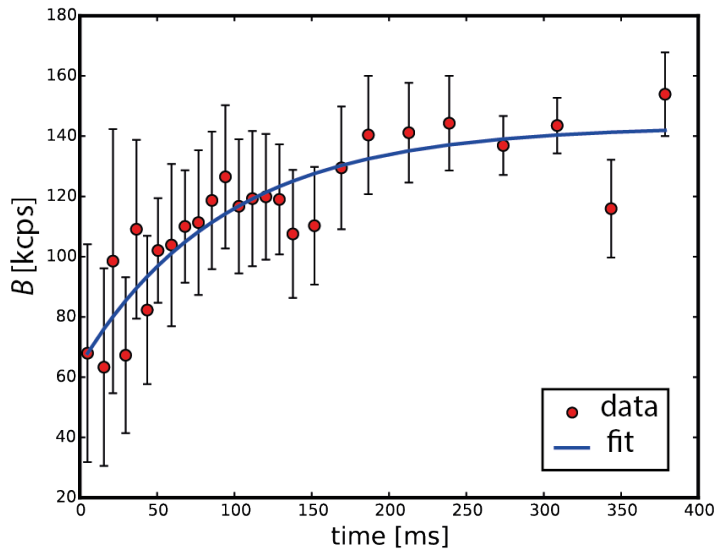

b.

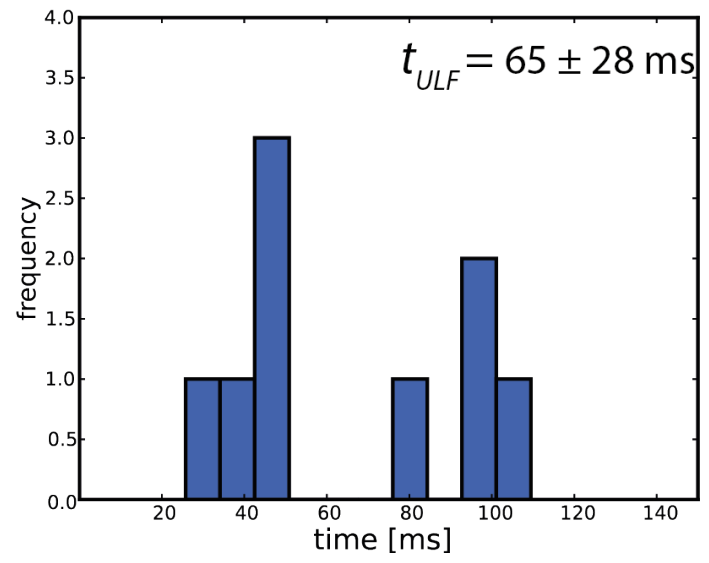

Figure 4.27: (a) Example of the exponential curve fit on a brightness curve over time for a vimentin assembly experiment. In this case the characteristic time is $93 \pm 27 \mathrm{~ms}$. (b) Histogram with all the time scales for vimentin lateral assembly.

protein. However, as for the experiment with vimentin tetramers, there is no definitive explanation of the cause of this second component yet.

Qualitative information about the dynamics of vimentin assembly can be retrieved from the behavior over time of the label stoichiometry. The formation of ULFs is a fast process and it happens at a time scale $\approx 100 \mathrm{~ms}$ from the starting of the assembly. On average, the octamer formation start immediately after the assembly is initiated and the hexadecamers units start forming around $40 \mathrm{~ms}$ after the 0 time point. To have quantitative information about the assembling dynamics, the curves of $B$ over time are fitted with an exponential growth model:

$$
B(t)=A\left(1-e^{-t / \tau}\right)+C .
$$

A typical example of the fit is reported in Figure 4.27a. $\tau$ can be interpreted as the time scale for the formation of the ULFs in vimentin assembly $\left(t_{U L F}\right)$. An average value of $65 \pm 28 \mathrm{~ms}$ is found for $t_{U L F}$. The average time scale is calculated taking into consideration 9 different vimentin assembly experiments with samples at different labeling ratios.

In Figure $4.27 \mathrm{~b}$, a histogram with all the time scales for vimentin lateral assembly is shown. The time scales for the formation of ULFs show some variability, in fact the values extend from 25 to $109 \mathrm{~ms}$. This may be related to flow fluctuations in the microfluidic devices. In particular, if the flow is not perfectly stable in the device, there may be changes in the positions of the vimentin stream and in the mixing position. Hence, uncertainties on the flow may lead to a mismatch of the calculated reaction time on the $x$-axis with the actual reaction time of the assembly. The time scale of vimentin assembly is also influenced by the protein concentration and the ion concentration [88], furthermore the fluorophore density may also affect the assembly dynamics. In particular, we previously shown, 
in Figure 4.22, that the assembly might be slower when the labeling ratio in high. Possibly, the dye molecules, depending on the orientation, may prevent the two vimentin sub-units to get in contact and assemble. If this is the case, we can expect that a higher number of fluorophores will have a larger effect on the assembly dynamics, which might be slowed down, than a lower number of labeled fluorophores. In fact, in the experiment where vimentin molecules are dialyzed with a LR of $40 \%$ (Figure 4.22), which is the highest LR tested during these measurements, the longest $t_{U L F}(109 \mathrm{~ms})$ between all the experiments is also obtained. This hypothesis is also supported by the reaction times of the two other examples reported in this section (Figure 4.21 and 4.21), where the time scales $t_{U L F}$ increase (from 26 to $45 \mathrm{~ms}$ ) with increasing labeling ratio (from 12 $\%$ to $28 \%$ ). The results obtained are discussed in more detail in the next section, where the quantitative results are compared with the current research on vimentin assembly.

\subsubsection{Discussion}

The results presented in this chapter show that $\mathrm{PCH}$ combined with microfluidics is a very suitable method to measure the first temporal steps of vimentin assembly. $\mathrm{PCH}$ is a very powerful technique to measure the brightness of fluorophores [8]. In particular, it is often employed to quantify the stoichiometry of biological samples [57], helping to measure the aggregation degree [63, 53], or to characterize a solution identifying the components by their brightness [56]. Thanks to the microfluidic device presented here, the early time points of protein assembly are accessible even with a slow measuring technique as PCH. In fact, to achieve good signal-to-noise ratio long acquisition times are needed [6]. The microfluidic device also acts as a mixer, bringing vimentin tetramers in contact with the assembly buffer [163. As soon as the assembly buffer is mixed inside the protein stream, the assembly reaction can be followed, with high temporal resolution, along the central channel of the device [14, 15, 16]. It is important to use a microfluidic device with a geometry that prevents clogging of the device [14]. Here a five-inlet geometry has been used with a height constriction (step) in the center channel which, combined with the proper flow rates, prevents assembled vimentin from touching the channel walls. When the step is not integrated into the microfluidic device vimentin assemblies, which are in contact with the top and bottom channel walls, are adsorbed on the surface, as shown in Figure 4.1. If the adsorption of vimentin is not prevented the protein concentration will change over time at each single spot of the device. Moreover the aggregates on the surface might interfere with the flow of the central jet, leading to, in the worst case, occlusion of the outlet channel [14]. This microfluidic device is easy to manufacture and to handle [68]. It is compatible with microscope acquisition since the bottom layer is constituted by a glass coverslip. We have shown that $\mathrm{PCH}$ is a reliable technique to measure the brightness of molecules in bulk and under flow at a single molecule level. As 
long as undersampling is avoided, we confirm that $\mathrm{PCH}$ is independent of the flow rates used 53 .

Measuring the brightness is a challenge. It is a measurement that depends on the configuration of the setup. Every small change, such as temperature changes or vibrations, influences the values measured [8, 51, 57]. It is not an absolute measurement, but the brightness measurements can only be analyzed in respect to a referee measurement [53, 57]. In this work, all the experiments in flow are compared with the bulk measurements of vimentin tetramers and of Atto-532, used as a baseline during the microfluidic experiments. When the monovalent ions are added to the flowing buffers, the assembly of vimentin is initiated [15]. An increase of number of labels per sub-unit is measured during the experiments, confirming vimentin assembly. The starting number of labels per sub-units measured at $t=0 \mathrm{~s}$ corresponds to the labeling ratio measured (in bulk) and predicted (with equation 4.1) for vimentin tetramers. The number of labels per vimentin sub-unit increases up to a value which corresponds to ULFs. In the time scale accessible with the step device, the assembly up to the formation of ULFs is followed, i.e. the vimentin lateral assembly, but there are no signs of filament elongation. The elongation reaction of vimentin protein into filaments cannot be measured since the accessible time scales with this device are on the order of seconds, while the elongation of the filament occurs in the order of minutes [89, 96]. The two-step vimentin assembly, first lateral and then longitudinal, was theoretically confirmed in [89] and in [90, where the authors mathematically modeled the assembly reaction kinetics with first, a very fast lateral assembly which consumes all the available tetramers in forming ULFs, and then, an elongation of the filaments which plays a role at longer time scales. To quantify the time scale of vimentin lateral assembly, the brightness curves are fitted using an exponential growth model. On average, after $65 \mathrm{~ms}$ from the initiation of the assembly, vimentin tetramers are assembled into ULFs.

We have shown that the number of labels per sub-unit measured during the assembly for a vimentin sample with low labeling ratio corresponds to the values predicted theoretically, as shown in Figure 4.21. In particular, the number of labels per ULF measured is comparable, within the experimental error, with the calculated value. When the labeling ratio increases, the value measured for ULF is slightly lower (Figure 4.20). One explanation of this discrepancy may be attributed to the high label density in ULFs, which possibly affects the brightness of single ULFs, decreasing the brightness actually measured. The fluorophores attached to the ULFs may interact with each other and the fluorescence signal could be self-quenched. A possible experiment to test if the decrease of the measured brightness can be attributed to quenching of the attached fluorophores would be to measure the lifetime of the dye molecules [133] at different positions in the microfluidic device. Higher labeling density might affect also the kinetics of vimentin lateral assembly, slowing down the process, as in Figure 4.22, where at $\mathrm{LR}=40 \%$ the ULFs value is not reached after $100 \mathrm{~ms}$ of assembly. It is known 


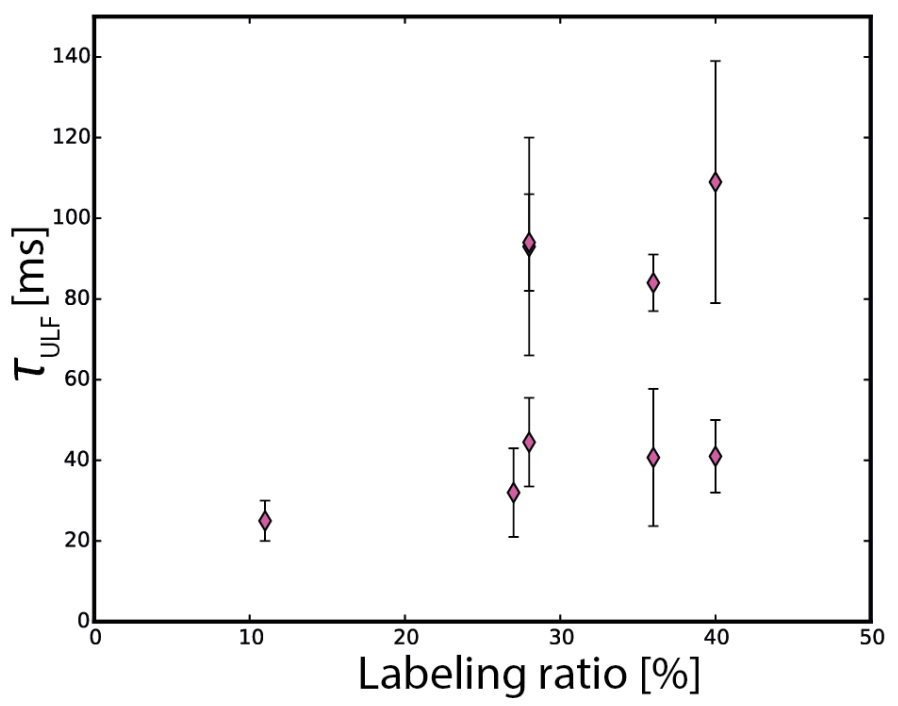

Figure 4.28: Relation between the time scales for the ULF formation and labeling ratio. At higher labeling ratios the time needed to assemble ULFs also increases. However, there are not enough data points to confirm or to model the relation between the LR and the time scales for the assembly.

that vimentin assembly is influenced by protein concentration and assembly conditions, thus also the labeling density might have an influence on the assembly dynamics [114]. In Figure 4.28 the time scales for the formation of ULFs are plotted against the labeling ratios. It seems that the assembly time increases at higher labeling ratio. Possibly, at high labeling ratio the lateral assembly is slowed down since the fluorophores may interfere with the interaction between two vimentin sub-units. However, we do not have enough data points to confirm the trend. Moreover, the data show some variability which may be caused by flow distortion inside the microfluidic device. Every small defect during the fabrication of the microfluidic devices influences the flow during the experiment, thus the flow streamlines will not perfectly match the simulated ones if defects are present. This may lead to errors in the calculation of the reaction time, which is the $x$-axis of the brightnesses curves, yielding, perhaps, to mistakes during the quantification of the lateral assembly time scale.

The elongation of the filaments was already measured with other techniques such as AFM [89, 91] or time-lapse EM microscopy [119, 88, 120]. It was shown in these papers how vimentin ULF elongates forming filaments, and how the assembly conditions such as ion concentration, type of ions in the assembly buffer, protein concentration and temperature affect the elongation dynamics. Vimentin filaments can be fully characterized by AFM and EM and the consequences caused by the different assembly condition can be directly compared [92]. However, these techniques cannot measure time scales faster than seconds. Our technique can be 
considered a complementary method that allows us to access the complete time scale of vimentin assembly, adding the early time points of the assembly to the global assembly dynamics of vimentin.

A similar approach to our method is described in [14, 15, 134, where microfluidics is combined with small angle x-rays scattering (SAXS) which is sensitive to small length scales on the order of 10 to $100 \mathrm{~nm}$. In these papers, the assembly of vimentin is measured quantifying the change in molecular mass during the assembly, which was initiated, as in our experiments, in a microfluidic device by ion diffusion. Since, with X-rays, the signal is proportional to the electron density of the sample, labeling the protein with fluorophores is not necessary. With X-ray techniques, however, to have good signal-to-noise ratios high concentrations of protein are needed for the measurements. In PCH low protein concentration are employed especially to avoid the detector artifacts [52. While for SAXS measurements a concentration around 2 to $5 \mathrm{~g} / \mathrm{L}$ of vimentin has to be used, for PCH a concentration of $0.0025 \mathrm{~g} / \mathrm{L}$ is enough to obtain a good signal-to-noise ratio. A high X-ray flux is needed to measure proteins in the microfluidic devices. The $\mathrm{X}$-ray beam has to be focused down to a size on the order of $\mu$-meters for measurements in microfluidic devices. However, the X-ray beam has still to have high intensity after the focusing to perform scattering measurements on weak scatters, such as proteins. Consequently, experiments are performed preferably with synchrotron light sources. The microfluidic devices have also to be adapted to $\mathrm{X}$-ray. Since PDMS is not suitable because it absorbs energy at relevant photon energy for X-rays, microfluidic devices are manufactured with other materials. UV-curable adhesive/Kapton and cyclic olefin copoymers devices have been positively tested and used to measure the early time points of vimentin assembly [134]. However, these devices are not straightforward to manufacture. In particular, the constriction of the central channel device, to prevent assembling vimentin adsorption on the channel walls, is not easily reproduced in large quantities. Despite the difficulties of X-ray measurements, these experiments confirmed that ULFs become visible earlier than $1 \mathrm{~s}$ after starting the assembly, as our measurements also confirmed [14.

The first steps of the elongation of vimentin filaments have been measure with time-resolved multi-angles DLS and SLS [87, 94], where the assembly time scale between seconds and minutes can be directly followed. These time scales correspond to length scales between $100 \mathrm{~nm}$ and $500 \mathrm{~nm}$ for vimentin assembly, which are not easily measurable by SAXS or light microscopy. In DLS the signal measured is given by the molecular weight of the system studied, thus fluorophores are not needed. It is a very powerful technique because it allowed us to quantitatively measure the kinetics of vimentin elongation, however with just DLS the formation of ULF can not be measured. The time needed in [87, 94] to mix vimentin solution with the ion solution and mount the sample was typically around $20 \mathrm{~s}$. This dead-time covers the lateral assembly step. Since, the assembly of vimentin is initiated by directly mixing in the measuring cell vimentin 
tetramers and $\mathrm{KCl}$ buffer, a heterogeneous number of sub-units intra-filaments is observed in these experiments. This variability of number of monomers per ULF depending on the assembly modality was already confirmed in [119. In fact, mass-per-length measurements based on STEM images show the dependency on the method to assemble the filaments: when the assembly is initiated by dialysis vimentin tetramers into the assembly buffer, the number of monomers per subunit along the filaments is more uniform than when the filaments are formed by directly mixing the salt buffer into vimentin [119].

The second approach that up until now has been able to quantify the early time points of vimentin assembly is presented in [96. In the paper, the authors show a combination of SLS, which gives access to the changes in the molecular weight, with stopped flow, which is employed to mix vimentin tetramers and assembly buffer, to effectively measure vimentin assembly. Vimentin tetramers are quickly mixed with the assembly buffer with turbulent flow and the change in mass is measured directly afterwards. The authors measured that ULFs are formed in the first $100 \mathrm{~ms}$ of the assembly. The time scale of ULF formation are actually comparable to the one measured by us with $\mathrm{PCH}$ in flow. In fact, in our experiments, on average, ULF are formed at $60 \mathrm{~ms}$ from the starts of the assembly. One difference between the two methods lies in the trick used to mix vimentin tetramers and ions. In our microfluidic device, the mixing is achieved with diffusion [163], a very similar approach to what is normally used when vimentin is assembled in vitro. In their experiments, the mixing is achieved with turbulent flow, very quickly, and this may lead to heterogeneity of the number of the vimentin sub-units per ULF [119]. The heterogeneity of vimentin sub-units intra-filament was actually measured and theoretically predicted with a model 96] in their paper. In our experiments, the heterogeneity of the sub-unit number intra-filaments is not measured, confirming that our gentle mixing method is closer to the standard conditions. The "polimorphisity" of vimentin might be potentially important in cells where the variability of the filament thickness might help to locally adapt the mechanical properties of the cell [135].

For now, the combination of microfluidics and PCH was only applied to study the assembly of vimentin, but in principle it can be applied to other molecular reactions. For example, it would be interesting to measure the early time points of keratin assembly, an IF that has even faster assembly kinetics than vimentin. The flow rates could be adjusted to zoom in on the very first time points of keratin assembly and to characterize, consequently, the dynamics involved. Or it could also be applied, for example, with $\alpha$-synuclein. For this protein, a controlled environment is fundamental since it changes conformation with even small changes in the measuring cell. Microfluidics then could be appropriate since it allows for a precise control on the solutions in flow. To conclude, the combination of microfluidics and $\mathrm{PCH}$ allows us to measure the early temporal points of vimentin assembly, and, in the future, could be used to measure the aggregation of many other molecules. 


\subsection{Protein interactions in the synapse}

In this section, experiments conducted to study protein interactions in synapses are presented and discussed. First, in cellulo FCS measurements of protein mobility in transfected neurons are shown. Second, a method to pattern SVs on glass surfaces is described. Interactions between $\alpha$-synuclein and synaptic vesicles are measured with FCS on patterned glass coverslips. Lastly, a microfluidic approach that allows to access synaptic dynamics is discussed.

\subsubsection{Measuring protein diffusion in living neurons}

The dynamic processes inside synapses are poorly understood. Even if some synaptic pathways are well researched, like for example synaptic vesicle recycling [110], the general dynamic organization of synaptic proteins is still enigmatic. Understanding protein mobility in the synapse is fundamental to properly interpret the function of the vesicles cluster during synaptic activity. Recently, a model proposed a liquid-liquid phase model describing the interacting synapsin with SVs [140]. Measuring the protein mobility in the synaptic cluster might confirm the model for other synaptic proteins also in living neurons. Measuring protein mobility in synapses of living neurons is challenging. The synapse is a complex and crowded environment, as shown in Figure 2.10, thus the interpretation of the data is not straightforward. A possible solution is fluorescence recovery after photobleaching (FRAP), which was used by our collaborator Sofiia Reshetniak in the research group of Prof. Silvio Rizzoli, to measure the mobility of 47 synaptic proteins. For the FRAP measurements, the proteins are expressed in mature hippocampal cultured neurons. A particular focus is put on proteins involved in exo- or endocytosis of SVs. These measurements propose themselves to be the first data to understand the synaptic protein diffusion in living neurons.

In FRAP, the fluorophores are intentionally bleached and the time needed to recover the fluorescence signal is measured. The recovery time is proportional to the diffusion of the specific targeted protein. FRAP recovery curves are fitted with an exponential growth function which provides information on the recovery time constant and the immobile fraction. Yet, the interpretation of the recovery times in terms of diffusion is not straightforward for FRAP experiments [136]. A realistic 3D model of the synapse, generated starting from EM microscopy data [141, 142], is employed to track synaptic proteins in 3D. The 3D synaptic model is conceived by Prof. Silvio Rizzoli. With the synaptic model, protein movements are simulated using a Monte Carlo approach, and the diffusion coefficients of the different proteins are calculated taking advantages of particle tracking analysis. With the 3D synaptic model, FRAP data are also simulated. The recovery time after a bleaching event is measured and in silico FRAP recovery curves are analyzed using the same fitting procedure as for the in vivo measurements. For every protein, the in silico FRAP recovery curves are compared with the correspondent 


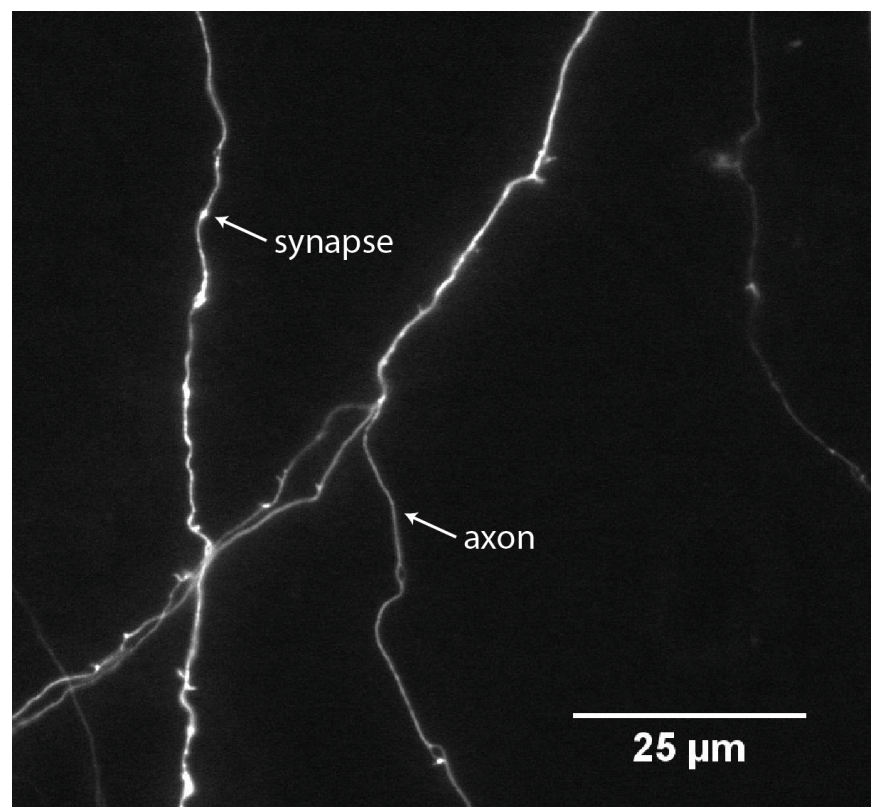

Figure 4.29: Typical epi-fluorescence image of hippocampal cultured neurons expressing mEGFP. A synapse and an axon are marked in the figure.

in vivo measurements. When the two match then, the diffusion coefficient, which is obtained by the model, is pinned to that particular measured protein, helping to have quantitative measurements of diffusion from FRAP data.

An alternative experimental technique is sought to compare the protein mobility measured with FRAP. FCS is commonly used to measure diffusion coefficients, thus, here, FCS is applied to measure synaptic protein mobility in living neurons. The coverslips containing the living neuronal cells in Tyrode's solution are mounted on the sample holder. Data at different positions within the axons and in different cells are acquired for each protein analyzed. For the FCS measurements, only neurons expressing mEGFP-SNAP25, EGFP-synapsin, soluble mEGFP and membrane-anchored mEGFP are analyzed. These proteins are chosen for the measurements because mEGFP-SNAP25 and EGFP-synapsin are common soluble proteins in the synapse, while soluble mEGFP and membraneanchored mEGFP are already well characterized when expressed in cells [148]. Epi-fluorescence images are used to identify the measuring position within the neuron and to focus on the chosen spot. A typical epi-fluorescence image of a neuronal cells expressing mEGFP is shown in Figure 4.29. The synapses are the high intensity spots situated sparsely along the axon.

FCS measurements on living cells are challenging due to artifacts which might be originated from the studied cells, such as auto-fluorescence background or intrinsic intracellular movements [147]. The concentration of the fluorophore cannot be controlled externally since it is determined during the expression of the protein of interest. Usually in cells the diffusion is highly confined and, with 


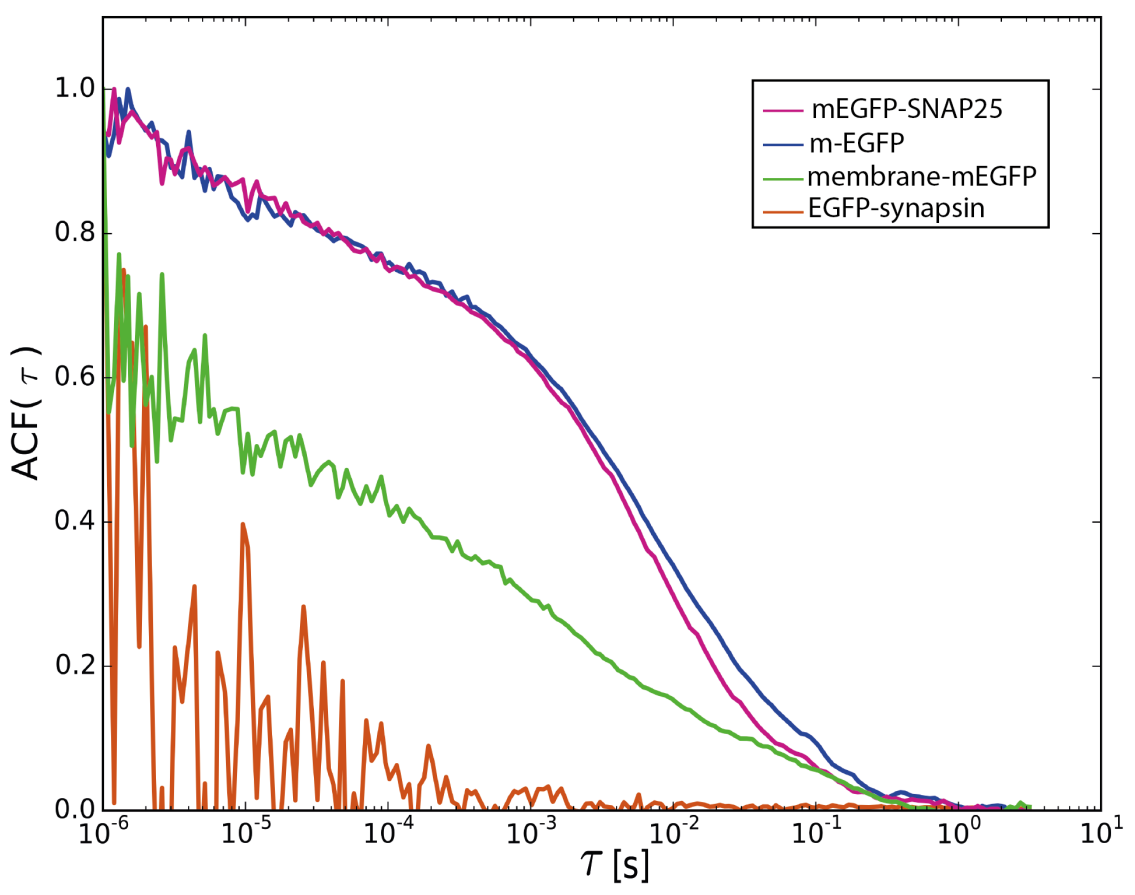

Figure 4.30: Normalized average FCS curves acquired on neurons expressing mEGFP-SNAP25, mEGFP, membrane-anchored mEGFP or EGFP-synapsin. Each curve is the average of at least 20 FCS runs, with acquisition times between 10 and $30 \mathrm{~s}$ for every run.

the high concentration measured in these samples, bleaching effects are common [147. Bleaching correction is not possible for all experiments, since usually too much bleaching occurs $(>50 \%)$ [36]. Data where pronounced photo-bleaching or large aggregates are visible, are excluded from the analysis. Figure 4.30 shows the normalized average FCS curves for the proteins measured in different positions along axons. All the FCS curves are analyzed with a 2D diffusing model [6], since the $z$-focus of the PSF is larger than the thickness of axons $(1.2 \mu \mathrm{m}$ compared to $300-500 \mathrm{~nm}$ ), and thus only diffusion in $x-y$ is taken into account.

For EGFP-synapsin, the high density of the protein and the pronounced bleaching effects dominate the measurements. A reasonable correlation of the data is impossible to achieve. For mEGFP-SNAP25 a diffusion coefficient of $D=$ $2.5 \pm 0.6 \mathrm{~mm}^{2} / \mathrm{s}$ is obtained in the proximity of the synapse, while a diffusion coefficient of $D=4.6 \pm 0.3 \mathrm{~mm}^{2} / \mathrm{s}$ is measured in the axon. A faster diffusion in the axon compared to the diffusion measured in the synapse is also recorded in most proteins measured with FRAP. On average, we find a diffusion coefficient of $D=$ $3.7 \pm 0.6 \mathrm{~m}^{2} /$ sin neurons expressing mEGFP-SNAP25. For membrane-anchored mEGFP an average diffusion coefficient of $D=1.4 \pm 0.4 \mathrm{\mu m}^{2} / \mathrm{s}$ is measured in the axonal section of the neurons. Neurons expressing soluble mEGFP show an 
average diffusion coefficient of $D=0.9 \pm 0.4 \mu \mathrm{m}^{2} / \mathrm{s}$. This value is approximately 20-fold below the diffusion coefficient [143] measured in the cytoplasm of Chinese hamster ovary $(\mathrm{CHO})$ cells. The difference between our measured diffusion coefficient and the literature value may also be attributed to the difference in the cellular environment between the $\mathrm{CHO}$ cells and neurons.

The FCS diffusion coefficients are surprising since the values are not in agreements with previous measurements. First of all, mEGFP is supposed to diffuse in the cytosol faster than mEGFP-SNAP25 and membrane mEGFP, while in our FCS measurements the diffusion coefficients are very similar to each other. Moreover, the value measured for SNAP-25 is about 10 times higher than expected from previous measurements in neuroendocrine PC12 cells [146]. Theses differences can be related to difficulties during the experiments and during the data interpretation. First of all, the measurements are acquired on living samples which are not completely characterized and controlled: e.g., the local intracellular viscosity at the evaluated position is unknown, as also the exact temperature in the sample. Diffusion measurements are particularly sensitive to change in temperature and while our measurements are acquired at a temperature of 21 ${ }^{\circ} \mathrm{C}$, the FRAP measurements were acquired at a temperature of $37^{\circ} \mathrm{C}$. Secondly, a fitting model describing $2 \mathrm{D}$ freely diffusing particles is chosen to analyze the data from all the proteins, however, it might have been not the most appropriate model. Since some proteins measured are also not diffusing freely but interacting and binding to other synaptic components, a model describing anomalous diffusion was also considered. However the diffusion coefficients obtained with the anomalous diffusion model are similar to the values from the $2 \mathrm{D}$ free model. It was already found in [137] that not always there is a need to invoke anomalous diffusion for in-cell diffusion measurements. The autocorrelation analysis seems to be dependent on the cell type measured, for example, simple diffusion of eGFP has been observed in cells [138, as well as anomalous diffusion behavior [139]. Other models have also been proposed to measure diffusion in the synapse, such as the so called "stick-and-diffuse" model or the "caged" model [149, in both cases the geometry of the system is taken into consideration to properly describe the FCS curves. In particular, the size of axons is comparable with the dimension of the observation volume in the axial dimension. This also applies to most synapses, their size is comparable with the PSF spot of the laser. The diffusion coefficients might have been underestimated depending on the mobility of the molecule. Thus, complementary measurements and sophisticated analysis would be necessary to reliable interpret the data in the context of proteins mobility in vivo measurements at the synapse.

\subsubsection{Measuring in vitro protein-vesicle interactions}

The experiments presented in this section are performed together with Sofiia Reshetniak and Prof. Silvio Rizzoli from the Institute for Neuro- and Sensory 


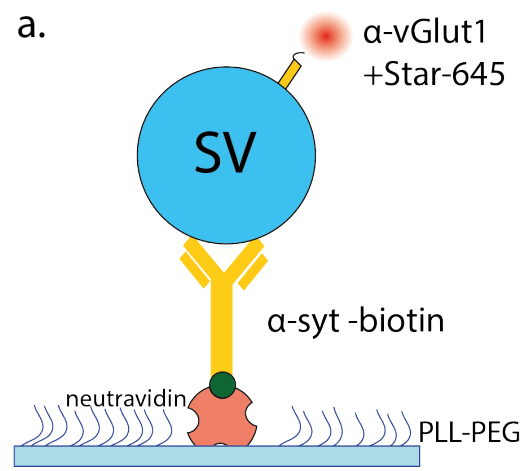

b.

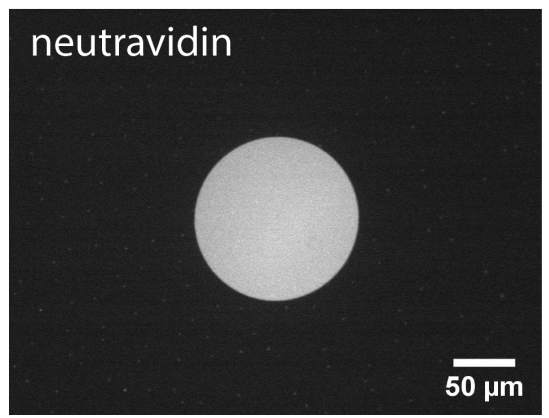

Figure 4.31: Sketch of the attachment protocol of SVs to glass coverslip and a typical image of a neutravidin pattern. (a) Neutravidin at a concentration of $0.05 \mathrm{~g} / \mathrm{L}$ is patterned on a cleaned glass coverslips with the PRIMO patterning system. Anti-synaptotagmin-1 biotinylated antibodies at a concentration of $0.01 \mathrm{~g} / \mathrm{L}$ are used to connect the SVs to the neutravidin patterns. The SVs are fluorescently labeled using anti-vGlut1 nanobodies tagged with STAR-635 fluorophores with a concentration of $0.005 \mathrm{~g} / \mathrm{L}$. (b) Typical epi-fluorescence image of a neutravidin pattern (neutravidin-FITC $\mathrm{C}=0.05 \mathrm{~g} / \mathrm{L}$ ).

Physiology in the University Medical Center of Göttingen. The proteins, the vesicles, the antibodies and the nanobodies employed for this work are provided by Sofiia Reshetniak. As shown in the previous section, measuring protein diffusion in vivo is challenging. Since the synapse is a complex and crowded environment, an in vitro simplified version of this biological complex is created to study protein mobility in a controlled manner. Using patterning techniques, SVs are attached to a glass coverslip and FCS measurements of synaptic proteins are performed on top of the SV pattern to study the interactions.

The SVs are attached to the glass surface with the protocol described in Section 3.2. Briefly, circles with a diameter of $130 \mu \mathrm{m}$ of neutravidin are patterned on a glass coverslips, using the patterning system PRIMO. A typical epi-fluorescence image of the patterns achieved is shown in Figure 4.31 b. Neutravidin-FITC at a concentration of $0.05 \mathrm{~g} / \mathrm{L}$ is used for visualization purposes, however for the actual measurements no fluorescent neutravidin is used. Anti-synaptotagmin1 biotinylated antibodies, diluted at a concentration of 1:100 in PBS $(c=0.01$ $\mathrm{g} / \mathrm{L}$ ), are employed to attach the SVs to the neutravidin pattern. The SVs are labeled through a FluoTag anti-VGLUT1-STAR635 nanobody, which recognizes the cytosolic domain of VGLUT1 present on the SVs, at a concentration of 1:500 $(c=0.005 \mathrm{~g} / \mathrm{L})$. Figure 4.31 a represents a sketch of the attachment strategy to create the SV patterns.

The attachment of the $\alpha$-syt-biotin antibody is tested incubating $\alpha$-ms-rvs- 
a.

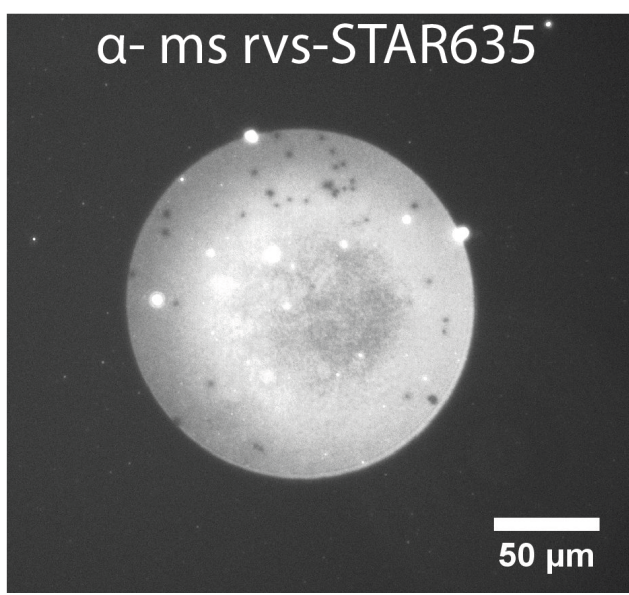

b.

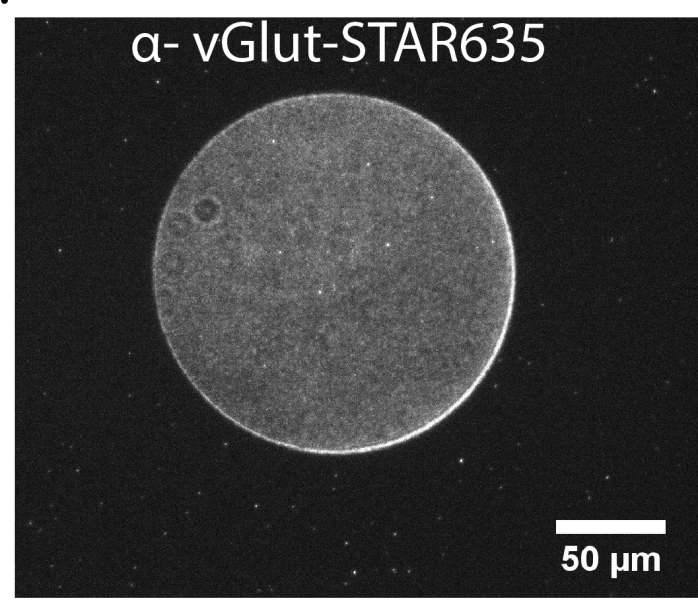

Figure 4.32: Epi-fluorescence images of (a) $\alpha$-ms-rvs-STAR635, which recognizes the $\alpha$-sytbiotin antibody, and (b) $\alpha$-vGLUT-STAR635, which recognizes the GLUT receptors on the SVs. In (a) the attachment of $\alpha$-syt-biotin antibody is tested and no SVs are added to the patterns. The picture in (b) represents a typical image of patterned SVs.

STAR635, diluted 1:100 $(c=0.01 \mathrm{~g} / \mathrm{L})$, on top of the patterns before adding SVs. $\alpha$-ms-rvs-STAR635 is a fluorescent nanobody which recognizes anti-mouse antibodies, in this case, the $\alpha$-syt-biotin attached to the neutravidin. Figure $4.32 \mathrm{a}$ shows a typical epi-fluorescence image of the $\alpha$-ms-rvs-STAR635 signal on the patterns. The attachment of the SVs is monitored with $\alpha$-vGLUT-STAR635, that allows us to visualize the SV patterns (Figure 4.32 b). The patterns are also tested with a positive and a negative control antibody. We use a fluorescent antibody which recognizes a typical protein domain of the SVs as positive control, i.e. to test if the patterns visible under the microscope are really patterned vesicles. When we clearly identify a pattern which overlays with the SV patterns, also in the antibody channel, then we confirm that indeed the patter is composed by SVs since the antibody will attached only to them. The negative control is used to check unspecific binding on the patterned surface. Since the negative control antibody does not recognize any domain of the vesicles, it should not be visible on the pattern. In the case of the negative control, however, neutravidin molecules are patterned with a method called micro-contact printing, since the test was done before the installation of the PRIMO system. The complete protocol for microcontact printing is reported in the Appendix A.1. Apart from the patterning of neutravidin, all the steps of the protocol to attach the SVs to glass surfaces are the same as before, thus we conclude that the negative control is reliable as well. First, the biotinylated $\alpha$-syt is tested again when the SVs are attached to it, using an $\alpha$-ms-Alexa532 antibody. As shown in Figure 4.33 a, the $\alpha$-ms antibody positively recognizes the $\alpha$-syt pattern. Second, the pattern of SV is tested with a fluorescent version of the $\alpha$-syt antibody, which is, this time, not biotinylated. 
$\alpha$-syt-Atto647 shows a fluorescent signal in correspondence to the SV pattern, as shown in Figure 4.33c. Figure 4.33b represents a typical fluorescence image of the SV pattern labeled with the nanobody $\alpha$-vGlut-STAR635. Finally, in Figure $4.33 \mathrm{~d}$ the negative control is shown. In this case a goat $\alpha$-rat antibody conjugated with Atto532 is employed as a negative control since it is expected not to recognize any molecule in our pattern. Note that the four images shown here are taken from different individual samples.

The first protein tested on top of the patterns is $\alpha$-synuclein $(\alpha$-syn), one component of the synuclein family, which is supposed to be involved in regulating fusion of SVs. Expressed in E. Coli, purified and labeled (Alexa-532) $\alpha$-syn is employed first, to test the experimental conditions. The positive ( $\alpha$-ms-Atto532) and the negative ( $\alpha$-rat-Atto532) antibody are also measured on top of the SV pattern. In Figure 4.34, the normalized average FCS curves for $\alpha$-syn-Alexa532, $\alpha$-ms-Atto532 and $\alpha$-rat-Atto532 diffusing on the patterned SVs are shown. The SV patterns are labeled using a secondary antibody labeled with Alexa-350, which recognizes a G96 antibody attached to the SVs. The nanobody $\alpha$-vGLUT was not used in this experiment, since the red fluorescence might interfere with the signal from the $\alpha$-syn-Alexa532. Labeling the SV patterns is fundamental because it allows focusing precisely on the plane of the vesicles, providing us to measure the diffusion on the SV layer. Furthermore, the same sample can be employed to evaluate the protein diffusion in area without SVs, allowing to estimate the bulk diffusion on the same sample.

$\alpha$-synuclein is measured also in bulk, without SV patterns, to have a baseline measurements of the diffusion coefficient. A diffusion coefficient of $D=102 \pm$ $20 \mathrm{\mu m}^{2} / \mathrm{s}$ (average done from 5 independent experiments, every experiment is composed by 30 acquisition runs, each run is has $30 \mathrm{~s}$ of acquisition time) is measured for free $\alpha$-synuclein in PBS. The obtained diffusion coefficient is in agreement with similar measurements performed on $\alpha$-syn monomers with FCS [150]. A hydrodynamic radius of $2.3 \pm 0.5 \mathrm{~nm}$ is calculated from equation 2.16 . Our value is similar to values in literature for the hydrodynamic radius of $\alpha$ synuclein measured with different techniques such as SAXS $\left(r_{H}=4 \mathrm{~nm}\right)$ [151] or FCS and DLS (from $r_{H}=1.9 \mathrm{~nm}$ to $r_{H}=3.5 \mathrm{~nm}$ ) [152].

When $\alpha$-synuclein is measured on the vesicle pattern, two diffusion coefficients are found. A first diffusion coefficient, with a value of $D=67 \pm 10 \mathrm{\mu m}^{2} / \mathrm{s}$, probably corresponds to the fraction of freely diffusing $\alpha$-syn. However, the diffusion coefficient is smaller than the value obtained in the bulk measurement. Since the measurements are acquired on the vesicles plane, free $\alpha$-synuclein molecules might have been slowed down by the patterned vesicles, in a similar way to what actually happens inside the synapse due to the crowded environment. The second diffusion coefficient has a lower value, $D=3.25 \pm 2 \mathrm{\mu m}^{2} / \mathrm{s}$, reflecting a lower protein mobility. We hypothesize that this component corresponds to $\alpha$-synuclein molecules bound to the SVs.

In the case of $\alpha$-ms-Atto532, which is the positive antibody, a similar behavior 


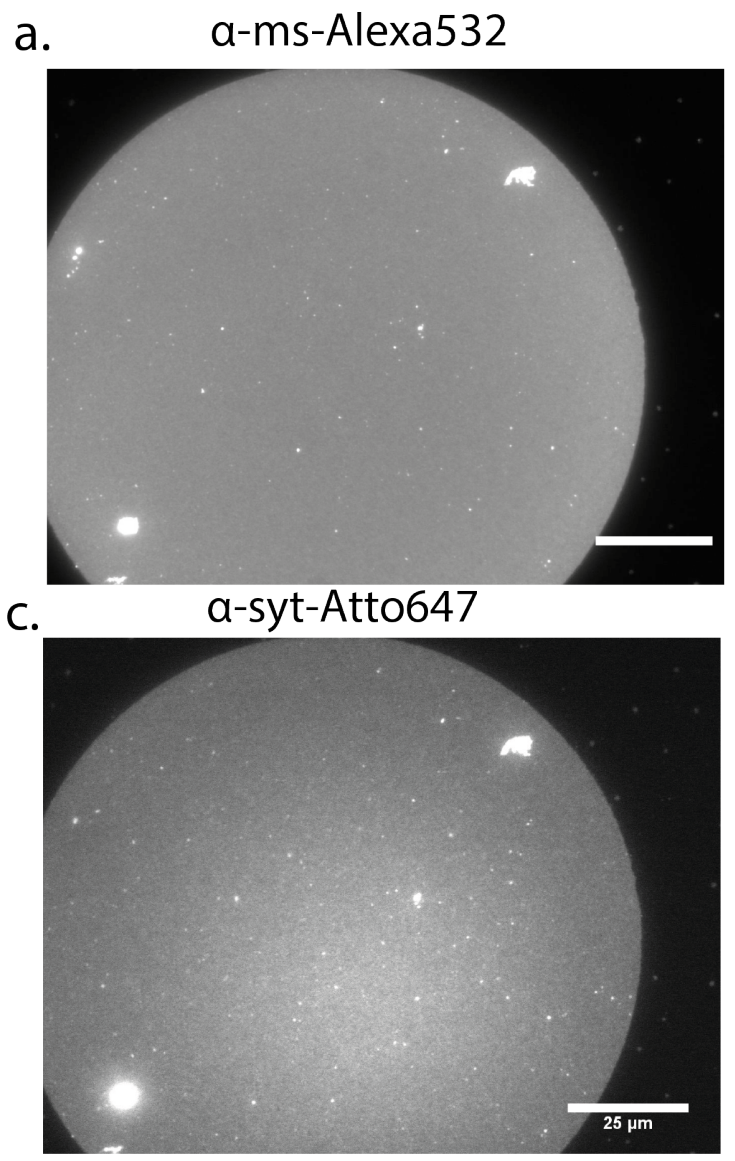

b. a-vGLUT-STAR635

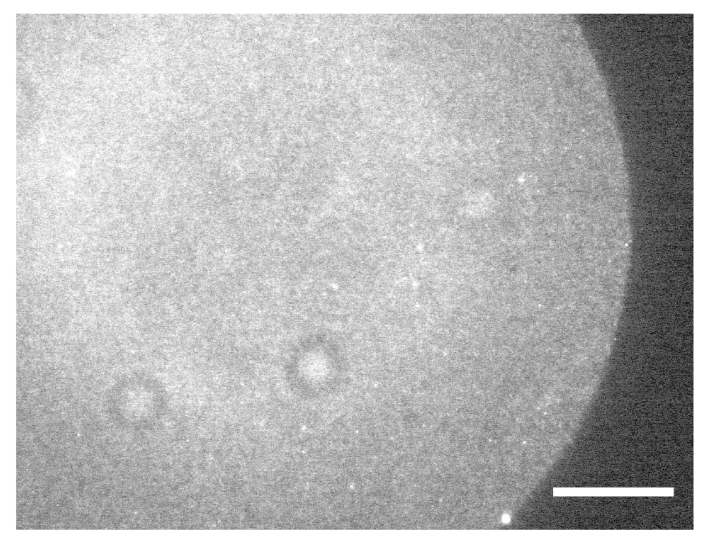

d. $\quad$ gt a-rat-Atto532

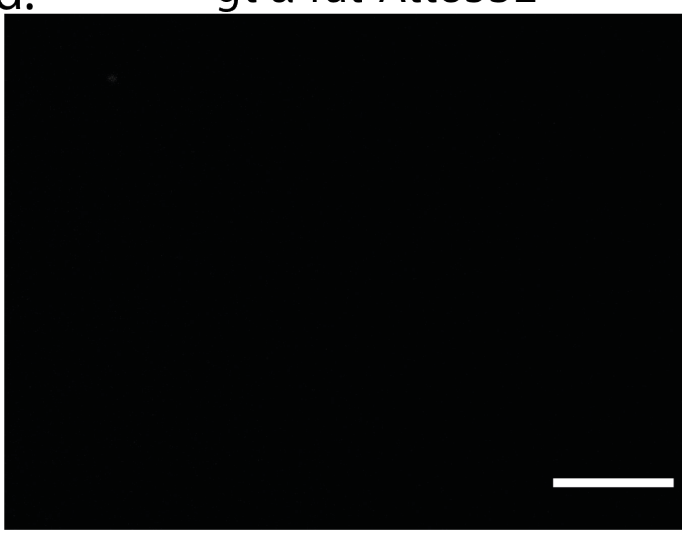

Figure 4.33: Epi-fluorescent images of different antibodies on the SV patterns. All these controls are done on glass coverslip with SVs attached. (a) Positive control on the $\alpha$-syt biotinylated antibody attached to the neutravidin pattern. (b) Nanobody attached to the SVs. (c) Positive control on the patterned SVs with a labeled $\alpha$-syt antibody. (d) Negative control on the SVs with a goat $\alpha$-rat (gt $\alpha$-rat) antibody which should not recognize any protein in our system. The negative test is conducted on an SV pattern created from neutravidin which was patterned using $\mu$-contact printing. The scale bar is $25 \mu \mathrm{m}$ in all images. 


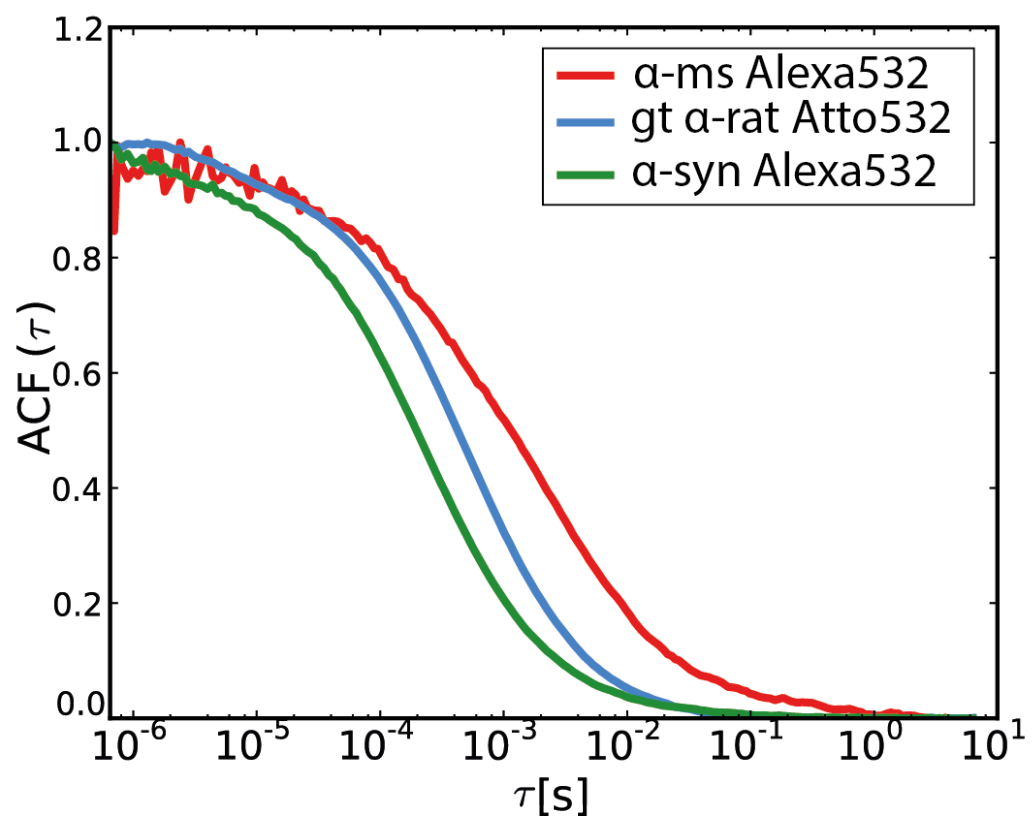

Figure 4.34: Normalized FCS curves of $\alpha$-synuclein-Alexa532, $\alpha$-ms-Atto532 and gt- $\alpha$-ratAtto532 measured on top of a SV pattern. Each curve is an average of 30 single FCS runs each of $30 \mathrm{~s}$. For $\alpha$-synuclein-Alexa532 two diffusion coefficients are measured: a "slow" diffusion coefficient $\left(3.25 \pm 2 \mathrm{~mm}^{2} / \mathrm{s}\right)$ which corresponds to the fraction of interacting proteins and a "fast" diffusion coefficient $\left(67 \pm 10 \mathrm{\mu m}^{2} / \mathrm{s}\right)$, which describes the freely diffusing component. The same behavior is obtained when the fluorescence signal from the positive control antibody, which interacts with SVs, $\alpha$-ms-Atto532 is acquired. One diffusion coefficient corresponds to the interacting antibody $\left(1.9 \pm 1 \mathrm{\mu m}^{2} / \mathrm{s}\right)$ and the other to the non-interacting component $(49 \pm$ $10 \mathrm{\mu m}^{2} / \mathrm{s}$ ). While in the FCS data from gt- $\alpha$-rat-Atto532, the negative control antibody, which is not supposed to interact with the SVs, a diffusion coefficient, corresponding to the fraction of freely diffusing antibodies is measured $\left(43 \pm 20 \mathrm{\mu m}^{2} / \mathrm{s}\right)$. Surprisingly a slower component is found also for this antibody $\left(8 \pm 5 \mathrm{\mu m}^{2} / \mathrm{s}\right)$, which possibly reflects the crowding effects of the patterned SVs. 


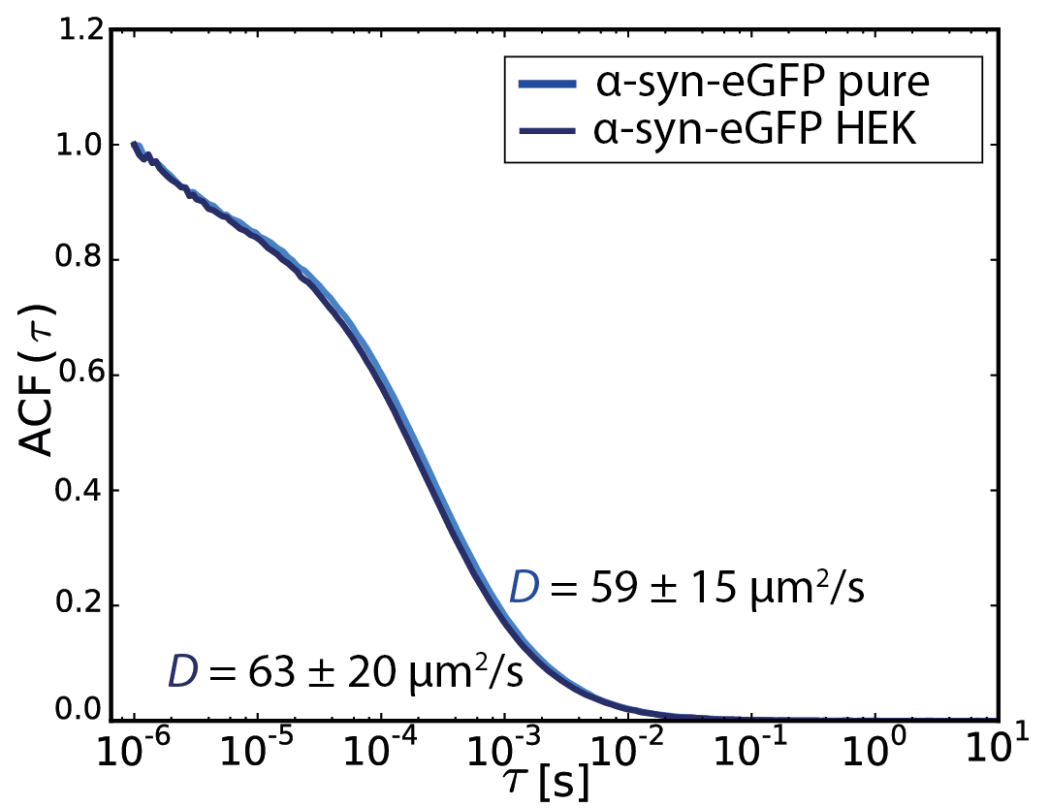

Figure 4.35: Normalize FCS average curves of $\alpha$-synuclein-eGFP purified and $\alpha$-synuclein-eGFP from HEK cell lysate freely diffusing in bulk. The two protein batches have a similar diffusion behavior, the purified $\alpha$-syn has a diffusion coefficient of $D=59 \pm 15 \mathrm{\mu m}^{2} / \mathrm{s}$ while the $\alpha$-syn from HEK cell lysate diffuses with $D=63 \pm 20 \mathrm{\mu m}^{2} / \mathrm{s}$. The two diffusion coefficients are compatible with each other within the measurement error.

as for $\alpha$-syn is expected. Indeed, two diffusion coefficients are measured also for this antibody on top of the patterns. A diffusion coefficient component which corresponds to the free antibody of $D=49 \pm 10 \mathrm{~mm}^{2} / \mathrm{s}$ (from bulk measurements: $D=55 \pm 20 \mathrm{\mu m}^{2} / \mathrm{s}$ ), and one component which corresponds to the interacting antibodies, with a lower value of $D=1.9 \pm 1 \mathrm{\mu m}^{2} / \mathrm{s}$. The last antibody measured, $\alpha$-rat-Atto532, is not supposed to interact with the SV pattern (negative control antibody). However, again two diffusion coefficients are measured. The free component, which has a value very similar to the previous antibody measured, diffuses with $D=43 \pm 20 \mathrm{\mu m}^{2} / \mathrm{s}$ (from bulk measurements: $D=47 \pm 29 \mathrm{\mu m}^{2} / \mathrm{s}$ ). Surprisingly, a slower component is measured also in this case, with $D=8 \pm$ $5 \mathrm{~m}^{2} / \mathrm{s}$. Possibly, this component describes the crowding effect caused by the vesicle pattern, which slows down the mobility of the antibody, or it might be caused by aggregation of the antibody in solution.

The diffusion coefficients presented here are the averages of three independent experiments each. For every experiment, each type of molecule has been measured between 20 and 60 times with an acquisition time of $30 \mathrm{~s}$ for each FCS curve acquired. The errors on the diffusion coefficients are high, reflecting the large variability of the experiments. In particular for $\alpha$-synuclein, which is a protein capable of many conformational changes, a large variability between measuring 
days is expected. The aggregation process of $\alpha$-synuclein is still largely unknown, however it can be initiated with changes in temperature [150, which could locally affect our experiments as well.

$\alpha$-synuclein labeled with eGFP is also measured on the SV patterns. In this case, the patterns are labeled with the vGLUT-STAR635 nanobody to avoid any bleed-through of the fluorescent signal. This batch of $\alpha$-synuclein is produced from HEK293 cells which are transfected with a plasmid for $\alpha$-synuclein-eGFP. The cells are harvested after some days to ensure the expression of the protein. Protein can be further purified for a cleaner sample or it can be measured directly after lysing the cells for a more "physiological" measurement. The purification of the $\alpha$-syn-eGFP is carried out by Sofiia Reshetniak and Prof. Silvio Rizzoli. The two protein batches are tested with FCS in bulk without SV pattern. Figure 4.35, shows the normalized FCS curves for the two $\alpha$-syn preparations. Each curve is an average of 30 FCS measurements with an acquisition time of $30 \mathrm{~s}$ for each single run. The two curves are normalized to the maximum value in each curve. The two $\alpha$-synucleins have a very similar mobility. The diffusion coefficients measured are comparable to each other, $D=59 \pm 15 \mathrm{\mu m}^{2} / \mathrm{s}$ for the purified $\alpha$ syn and $D=63 \pm 20 \mathrm{\mu m}^{2} / \mathrm{s}$ for the $\alpha$-syn from HEK cell lysate. First of all, on average, the diffusion coefficient measured for $\alpha$-syn-eGFP is slower compared to the one measured for $\alpha$-syn-Alexa532, this can be related to the size of eGFP molecule attached to $\alpha$-syn. Since eGFP is a bigger molecule (MW $=27 \mathrm{kDa}$ for eGFP and MW $=14 \mathrm{kDa}$ for $\alpha$-syn), the diffusion coefficient is slower than the one measured with a chemical dye as label. For both $\alpha$-syn-eGFP purified and in HEK lysate, a second diffusion component is always measured. This component has a high diffusion coefficient $\left(D=370 \pm 100 \mathrm{~m}^{2} / \mathrm{s}\right)$, compatible with a fast movement within the $\alpha$-synuclein and eGFP complex [145, 144].

Apparently, the two $\alpha$-synuclein batches (the purified $\alpha$-syn and the one measured directly in the cell lysate) are the same, however they behave differently on top of SV patterns. The $\alpha$-synuclein in the HEK cell lysate interacts less with the SVs. Possibly, some components in the cell lysate prevent the interaction between vesicles and $\alpha$-syn. Figure 4.37, shows epi-fluorescence images of the patterned sample during the experiment. In this case, $\alpha$-synuclein is washed with PBS after 2 hours of incubation on the SV patterns. When the $\alpha$-syn from the cell lysate is measured, no pattern is visible in the green channel. For this experiment, a diffusion coefficient of $50 \pm 10 \mathrm{\mu m}^{2} / \mathrm{s}$ is obtained, which is similar to the diffusion coefficient found for the freely diffusing $\alpha$-syn-eGFP. When the purified $\alpha$-syn is used in the measurements, the same SV pattern is visible also in the green channel, suggesting an interaction between the vesicles and $\alpha$-synuclein.

Figure 4.36, shows two normalized FCS curves for purified $\alpha$-syn-eGFP diffusing with or without SV patterns. The curves are the average of 20 single FCS curves, each acquired for $30 \mathrm{~s}$. Looking qualitatively at the curves in Figure 4.36. $\alpha$-syn-eGFP on the SV patterns seems to diffuse in general more slowly than the free $\alpha$-syn-eGFP, as expected. 


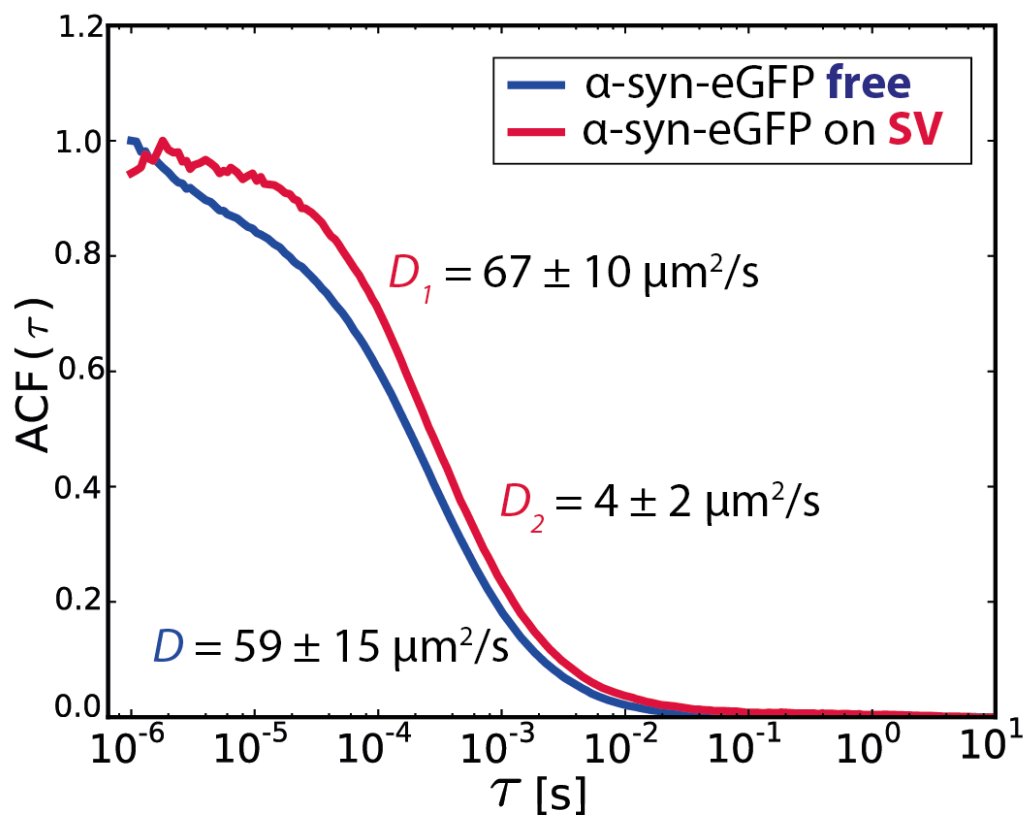

Figure 4.36: Normalized FCS average curves of $\alpha$-synuclein-eGFP freely diffusing in bulk and diffusing on top a SV pattern. The two FCS curves show some expected dissimilarity which reflects the different mobility of the proteins in the two experiments. In bulk, $\alpha$-synuclein is supposed to diffuse as a globular protein, as it should be in the unfolded state. When $\alpha$ synuclein is incubated on top of the SV pattern, a smaller additional diffusion coefficient is obtained, which corresponds to $\alpha$-synuclein bound to SVs. 


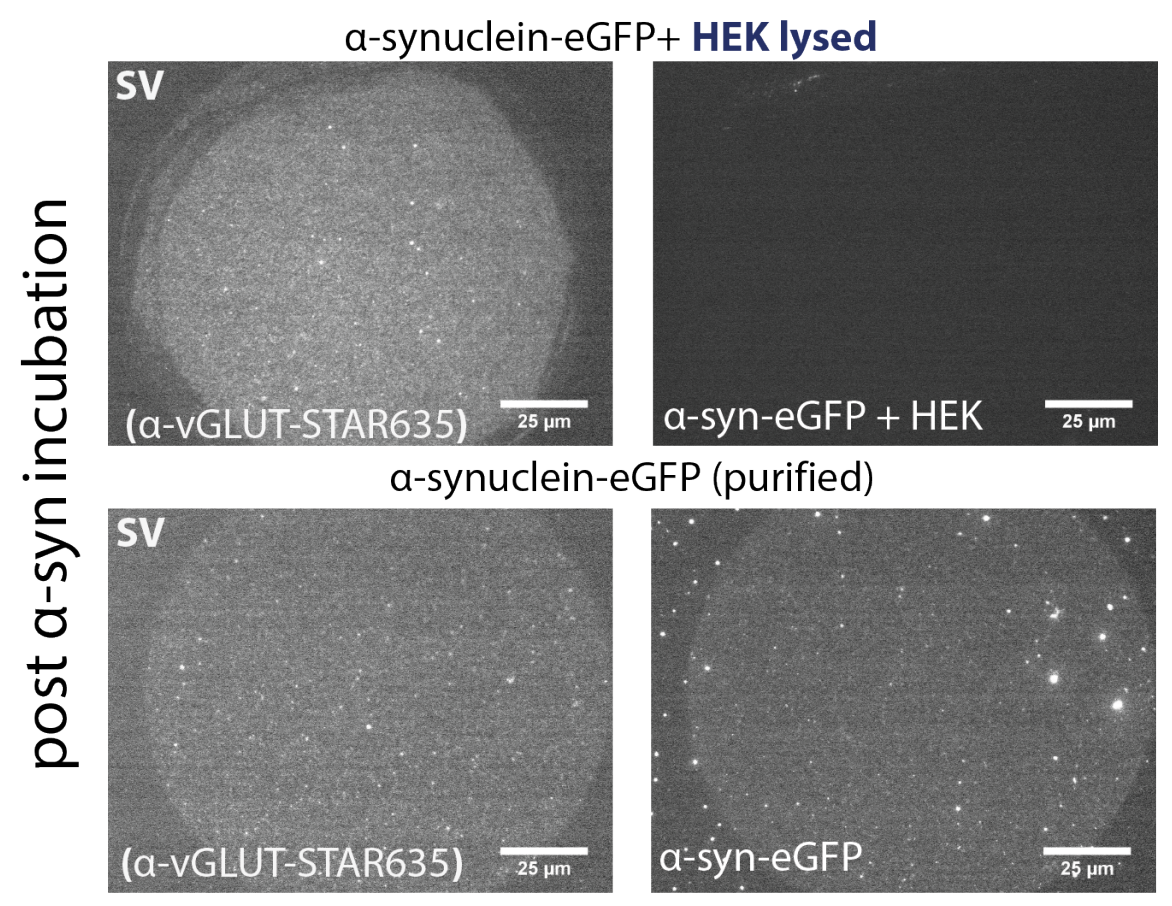

Figure 4.37: Epi-fluorescence images of SV and $\alpha$-synuclein patterns taken after FCS measurements. In this experiment, $\alpha$-synuclein is washed from the SV patterns after about 2 hours of incubation. FCS measurements are acquired also after the washing step to reduce the freely diffusing component. When $\alpha$-syn from the cell lysate is employed, no pattern is visible in the green channel, suggesting that there is no interaction between the vesicles and $\alpha$-syn. In the case of the purified $\alpha$-syn, the same pattern is visible in the green channel and in the red channel, suggesting an interaction between the vesicles and $\alpha$-syn. The two SV patterns are prepared with same protocol. 
When $\alpha$-synuclein is measured on top of the SV patterns, two diffusing components are measured. One is related to the free component of $\alpha$-synuclein still in solution, which diffuses with $D=67 \pm 10 \mathrm{\mu m}^{2} / \mathrm{s}$. The second component has a much slower diffusion coefficient of $4 \pm 2 \mu \mathrm{m}^{2} / \mathrm{s}$. This component represents the $\alpha$-synuclein molecules which have interacted with the patterned vesicles. The diffusion coefficient measured for the interacting $\alpha$-syn-eGFP is very similar to the one determined for the $\alpha$-syn-Alexa532 on top of the vesicles. The errors represent the standard deviation. In this case 10 independent experiments are averaged to obtain the mean value.

The errors are high, especially for the interacting component, because a large variability of diffusion coefficient is measured. In particular, the diffusion coefficient of the interacting component varies between 0.2 and $9 \mathrm{\mu m}^{2} / \mathrm{s}$. The variability can be explained by taking all the different possible configurations of $\alpha$-synuclein in consideration. Specifically, $\alpha$-synuclein changes its configuration from a globular intrinsically disordered conformation in solution to an elongated $\alpha$-helix, when bound to lipid surfaces [107]. The conformational change can influence the diffusion behavior of the protein when attached to the SVs. Depending on the local surrounding, the $\alpha$-helical structure of $\alpha$-synuclein might be more constrained than in other parts of the patterned SV area, thus movement could be slowed down. It has been showed that the binding affinity of $\alpha$-synuclein is affected by the lipid composition and the lipid curvature [153]. In particular, there is at least a 15-fold increase in affinity when small unilaminar vesicles are used instead of large unilamellar vesicles. However, in our measurements, the same type of vesicles, from the same purification batch, are used in all the experiments. Aggregation of $\alpha$-synuclein is not expected since low concentrations are used for the FCS measurements (between 2 and $50 \mathrm{nM}$ ). However, since $\alpha$-synuclein can change conformation and aggregates under various condition, aggregation induced by the vesicle interaction cannot be completely excluded.

More measurements have to be performed to have a clearer picture of the interaction between $\alpha$-synuclein and SVs. For example, measurements at different concentrations of SVs would elucidate if and how SVs hinder diffusion of the interacting $\alpha$-syn. Furthermore, measuring interactions between SVs and $\alpha$-synuclein in a buffer, which resembles physiological conditions, might be interesting in order to compare these data with the in vivo FRAP data presented in Section 4.2.1. Moreover, the interactions between SVs and aggregating $\alpha$-syn could be measured since the presence of ions in the buffers. It would be interesting to study how the interaction between vesicles and $\alpha$-syn changes when higher orders of $\alpha$-synuclein are formed.

\subsubsection{Synaptic vesicles under switchable flow}

This project is a collaboration with Helena Maria (Linda) Olsthoorn and Prof. Reinhard Jahn from the Laboratory of Neurobiology of the Max Plank Insti- 
tute for Biophysical Chemistry in Göttingen. A microfluidic device is created to enable controlled measurements of SV interactions under different flowing solutions. We are particularly interested in studying the neurotransmitter uptake by the vesicles. Even though endo- and exocytosis of vesicles are well studied, it still remains elusive how vesicles can be quickly filled with thousands of molecules. It is known that the neurotransmitter uptake by the SVs is achieved creating an electrochemical gradient on the vesicles membrane [154]. Vesicle acidification is difficult to study in cells due to the complexity of the synaptic environment and the fast dynamic processes involved.

Microfluidics helps to achieve temporal resolution, even with "slow" techniques. The microfluidic device employed allows for a fast switching of the solution flowing on top of a SV pattern located in the center of the outlet channel. In this way, dynamic processes, such as vesicles acidification, can be investigated with temporal resolution. The device can be employed to kinetically study vesicle reactions to different consecutive buffers. The SVs are patterned to the glass coverslip with a strong covalent bond to be able to survive the shear rates in the microfluidic device.

The microfluidic device is described in Section 3.3.2. Briefly, it is a three inlet and one outlet-channel device with a channel width of $1000 \mu \mathrm{m}$ and an height on $100 \mu \mathrm{m}$. This aspect ratio is chosen to achieve a plug flow profile in the device. As for the device shown in Figure 4.14, the velocity is constant along the width of the channel. In order to be able to switch the solution flowing on top of the pattern in the central channel, the side inlets are not pumping the fluids at constant velocity, but with a rectangular flow protocol, as shown in Figure 4.38a. The rectangular input flow profile applied to the side inlets have a periodicity of $20 \mathrm{~s}$ and they are anti-synchronized to assure a constant velocity in the central part of outlet, which is where the SV pattern is situated, as shown in Figure 4.38 b.

FEM simulations are employed to measure the response time of the system. The time needed to switch the solution on the SV pattern from one inlet to the other is considered as the response time. The simulations are performed using water as solution for the central channel and fluorescein is employed for the other two inlets $\left(D=425 \mathrm{\mu m}^{2} / \mathrm{s}[156]\right)$. The initial fluorescein concentration is 55 $\mathrm{mmol} / \mathrm{m}^{3}$. In Figure 4.39 the concentration of the solute from the side inlet 1 is shown at different time points for a simulation performed with the flow profiles from Figure 4.38. At time zero, the fluorescein solution from inlet 1 is flowing with the maximum velocity. At the same time, the solution from inlet 2 has the minimum velocity. At this time, fluorescein has the highest concentration in the center of the outlet. When the velocity of inlet 1 decreases, also the concentration starts to decrease in the center of the outlet. The fluorescein stream moves up towards the channel walls. At $t=10 \mathrm{~s}$, the fluorescein from inlet 1 flows with the minimum velocity, while the solution from inlet 2 flows with the maximum velocity. As consequence fluorescein concentration is zero in the center of the outlet. If the concentration of the solution from inlet 2 was followed, the 
a.

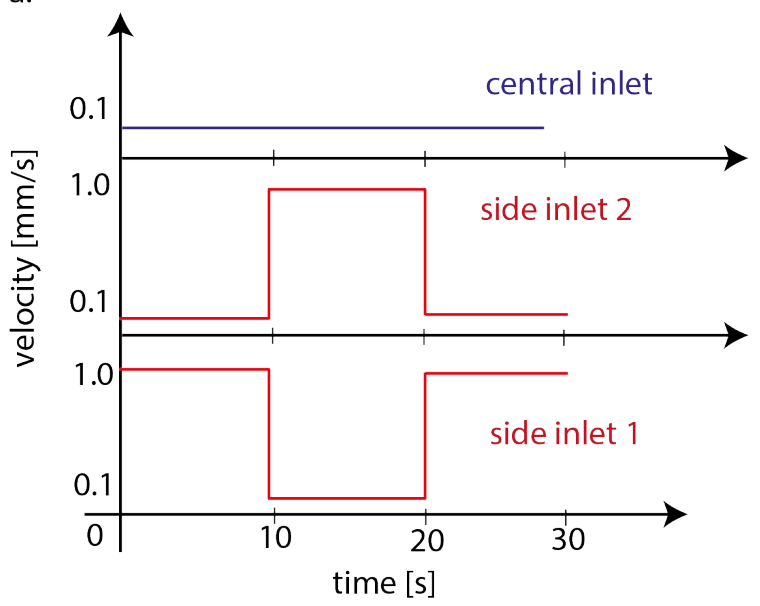

b.

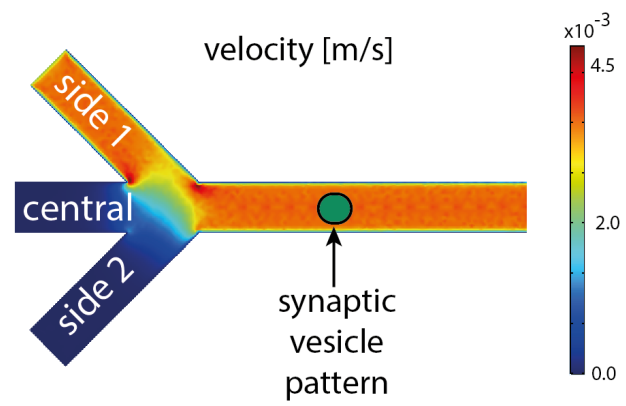

Figure 4.38: Input velocity profile applied to the inlets in the microfluidic device employed to switch buffers on the SV patterns. (a) Input velocity profile applied to the three inlets of the microfluidic device. In the central channel a constant velocity is applied. In the side inlets the solutions are pumped with a periodic anti-synchronized rectangular function. (b) FEM simulation of the flow velocity in a device with a width of $1000 \mu \mathrm{m}$ and an height of $100 \mu \mathrm{m}$ microfluidic device. The velocity is constant on the SV pattern due to the profiles of the velocity functions applied to the inlets.

behavior would be exactly the opposite. A zero value for the concentration will be recorded at $t=0 \mathrm{~s}$, an increase in the concentration, and then maximum value for concentration will be measured at $t=10 \mathrm{~s}$.

Measurements using the microfluidic device were acquired for comparison with the FEM simulations by a Bachelor's student, Sebastian Smyk. As in the simulation, fluorescein is employed as sample solution. In Figure 4.40, the epifluorescence images of fluorescein in the device are shown at different time points. From the microscopy images, the fluorescence intensity in a region of interest (ROI) (200x200 pixels) in the center of the outlet channel is determined as function of time, as shown in Figure $4.40 \mathrm{~b}$. The intensity curves are analyzed and compared to the simulations. To quantitatively extract the reaction time, the increase over time of the intensity signal in the ROI is fitted using a sigmoidal function. The measured response time in the experiments with a microfluidic device of $1000 \times 100 \mathrm{\mu m}^{2}$ is $0.462 \pm 0.008 \mathrm{~s}$. In the FEM simulations the response time is $0.34 \pm 0.01 \mathrm{~s}$, which is comparable to the measured value. To decrease the time, a smaller microfluidic device can be used. When a microfluidic device of $500 \times 50 \mathrm{\mu m}^{2}$ is employed, the response time decreases to a value of $0.255 \pm 0.005$ $\mathrm{s}$ in the experiment, while in the simulation, at the same conditions, the response time is $0.250 \pm 0.007 \mathrm{~s}$. However, the central channel needs to be sufficiently wide since a large area of SV is desirable for the experiments.

As in this case, SV patterns are on the glass coverslip that is the bottom of the microfluidic device, plasma treatment cannot be used to close the device, 


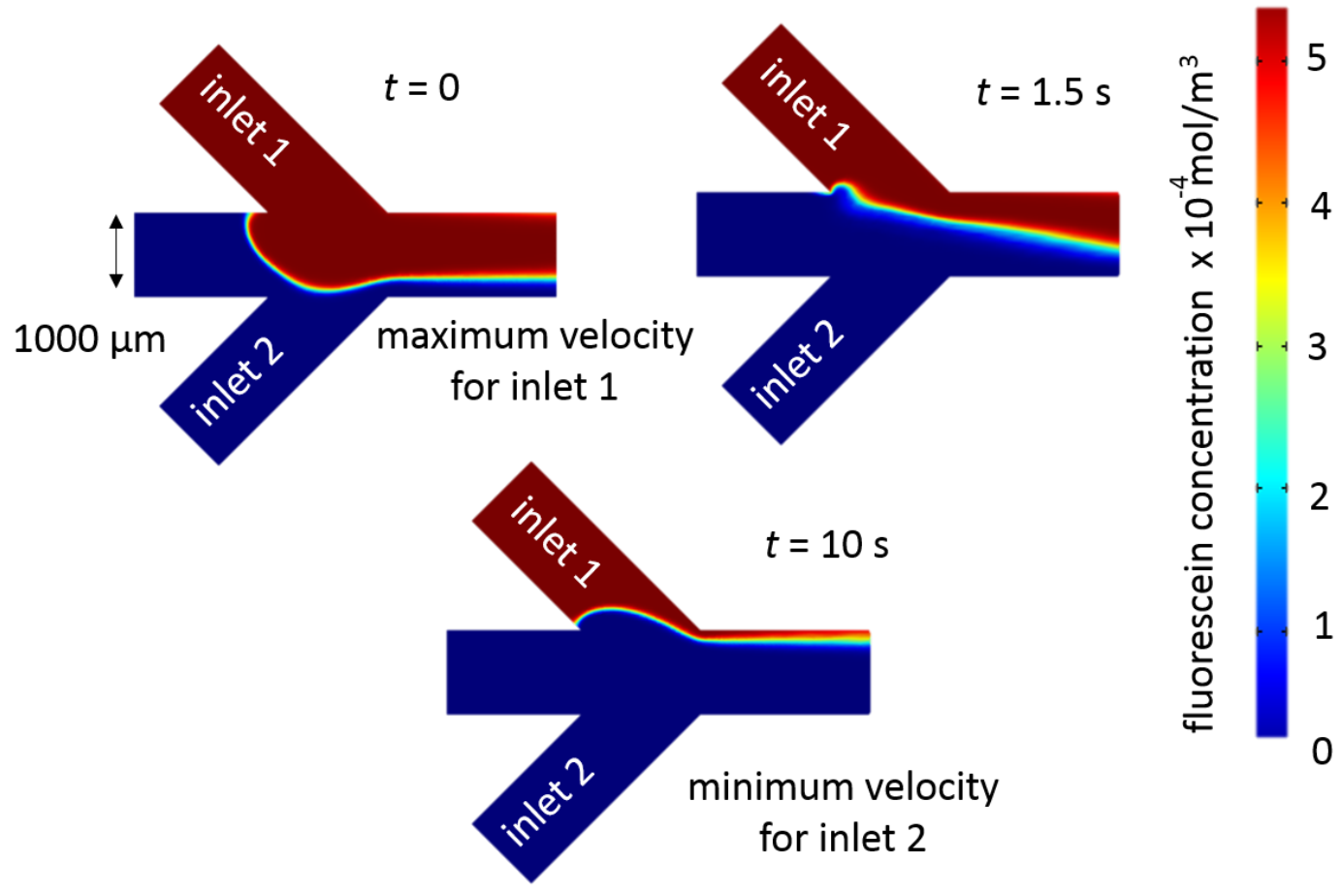

Figure 4.39: Concentration of fluorescein in simulated microfluidic device at different time points. The velocities employed in the FEM simulation are the same described in Figure $4.38 \mathrm{a}$. When the fluorescein from the top inlet is flowing with the maximum velocity, the flow from the bottom inlet is flowing with the minimum velocity. In this case, at $t=0 \mathrm{~s}$ in the center of the outlet the fluorescein from the top inlet has the maximum concentration. When the velocity from the top inlet decreases, the concentration in the center of the outlet also decreases. At $t$ $=10 \mathrm{~s}$ the fluorescein from the top inlet flows with the minimum velocity, as consequence the fluorescein concentration in the center of the outlet channel is null. 

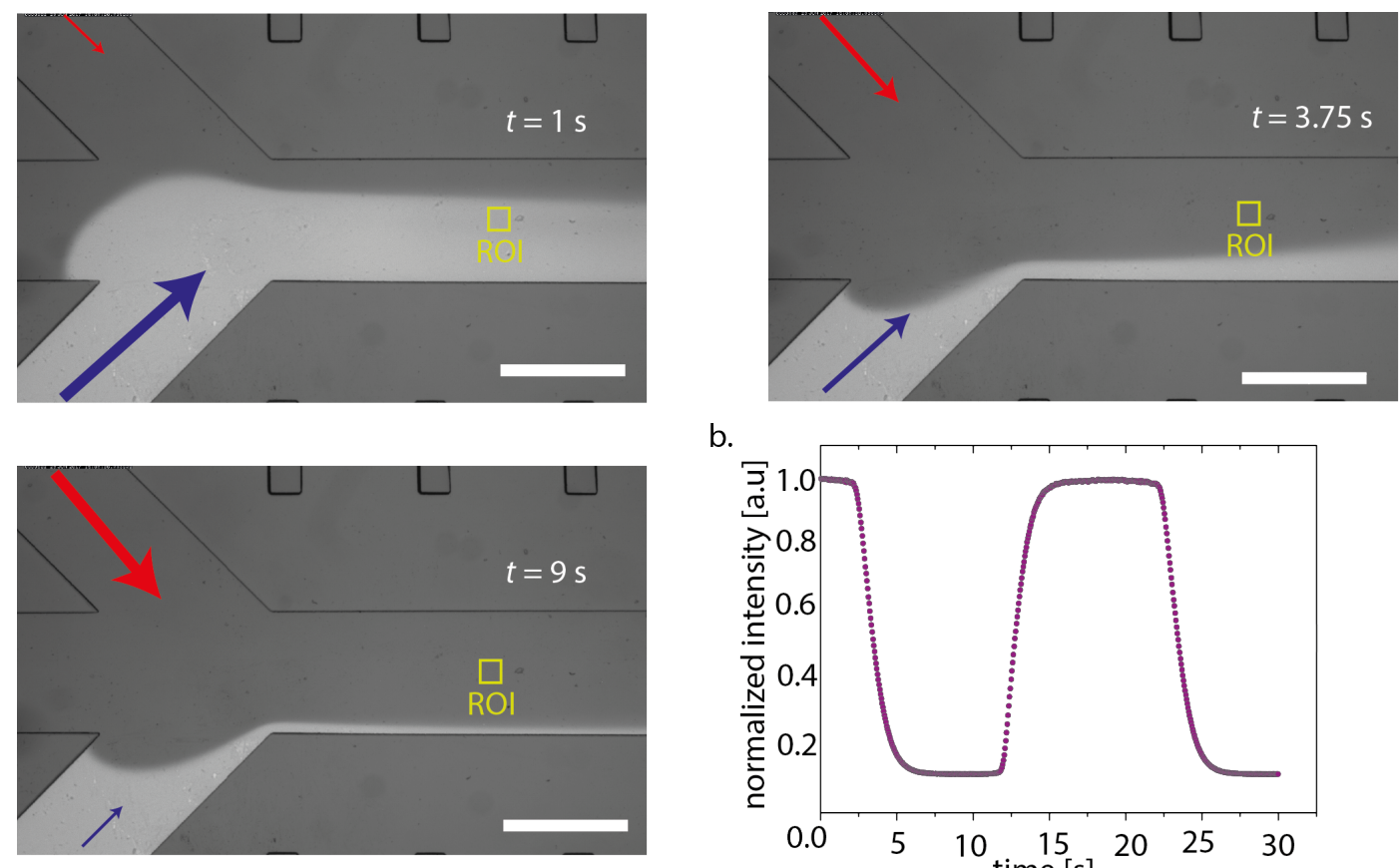

b.

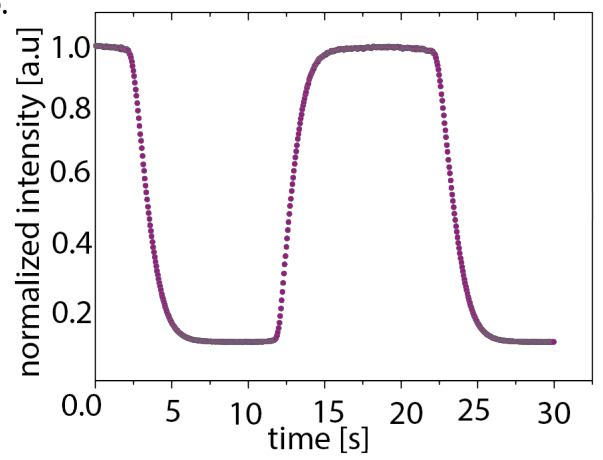

Figure 4.40: Epi-fluorescence images of fluorescein flowing in a microfluidic device with switching flow. The epi-fluorescence images are overlaid with the bright field image to show the device geometry. As expected from the simulations, the concentration of fluorescein in the center of the outlet is higher when the solution flows at with the maximum velocity (big blue arrow). As the velocity decreases, the solution moves away from the center of the outlet and the concentration decreases (small blue arrow). The arrows represent the magnitude of the velocity in the channels (bigger arrow $=$ higher velocity), red for the top inlet and blue for the bottom inlet. (b) Normalized fluorescence intensity over time averaged in a ROI (showed in yellow) in the center of the outlet channel. The intensity behaviour reflects the velocity flow profiles used in the measurements. The velocities and the concentration employed in the experiment are the same as in the simulation. The scale bars are $1000 \mu \mathrm{m}$ The experiment was performed by Sebastian Smyk. 
otherwise the SVs would be destroyed by the plasma. Thus, a custom-made device holder, inspired by [118, 134], is exploit to clamp the device preventing any leakage. A schematic representation of the device holder is shown in Section 3.3.2. Initially, the SVs are attached to the glass surface through antibodies (mouse ab anti-Synaptophysin1), covalently bound to epoxy coated glass surface. However, the vesicles detach from the glass surface when flow is applied. After 30 minutes of PBS buffer flowing on top of the SV pattern in the microfluidic device, with the same velocity of the simulations, the SVs are mostly detached from the surface, as shown in Figure 4.41. There is a clear difference between the controls and the patterns which are flushed in the microfluidic device. This problem has two possible causes. First, probably not all the synaptophisin antibodies are covalently bound to the surface, but the large majority is only adsorbed by it. Thus, when flow is applied, due to the shear stress on the antibody and SV complex, the molecules detach from the surface. The second cause can be the low density of binding site for the antibodies onto the epoxy surface.

The shear rate, for fluids in a pipe, is defined as the gradient of the velocity measured across the height of the microfluidic device as:

$$
\gamma_{S}=\frac{\partial \vec{v}_{i}}{\partial x_{j}}+\frac{\partial \vec{v}_{j}}{\partial x_{i}} .
$$

Since the velocity at the channel wall is zero, the shear rate is maximum close to the channel walls and then decreases. We can define the shear stress at a surface element parallel to a flat plate at the point $\mathrm{z}$, which for Newtonian fluids is given by:

$$
\tau_{S}(z)=\mu \frac{\partial \vec{v}}{\partial z}
$$

with $\mu$ the dynamic viscosity of the solutions and it is measured in $\mathrm{Pa}$ (dimensionally the shear stress is force per area). It can be considered as a measurement on how "strong" the flow is on the interested surface. In our case, we can use the shear rate to obtain a qualitative idea on the flow forces on the SV pattern. The shear rate can be evaluated for the FEM simulations. For the microfluidic device used here $\left(1000 \times 100 \mathrm{~mm}^{2}\right)$ with velocities as in Figure $4.38 \mathrm{a}$, a shear rate of $1701 / \mathrm{s}$ is measured in the center of the outlet channel. The reaction to the shear stress depends on the particle type and size under the flow [157]. In fact, previous measurements, where only the antibody was bound to the surface, show a less pronounced detachment of the molecules from the glass coverslip when flow is applied. In Figure 4.42, epi-fluorescence images of the antibody patterns before and after 30 minutes in the microfluidic device with PBS flowing at a velocity of $4 \mathrm{~mm} / \mathrm{s}$, are shown. The patterned antibodies are visualized thanks to a secondary antibody labeled with Alexa-532 (goat $\alpha$-mouse-Alexa532). The controls are mounted in the device holder, but no flow was applied to the patterned antibodies. Two different samples with synaptophisin 1 are tested in the microfluidic 


\section{a. controls (untouched by device)}

\section{b.}
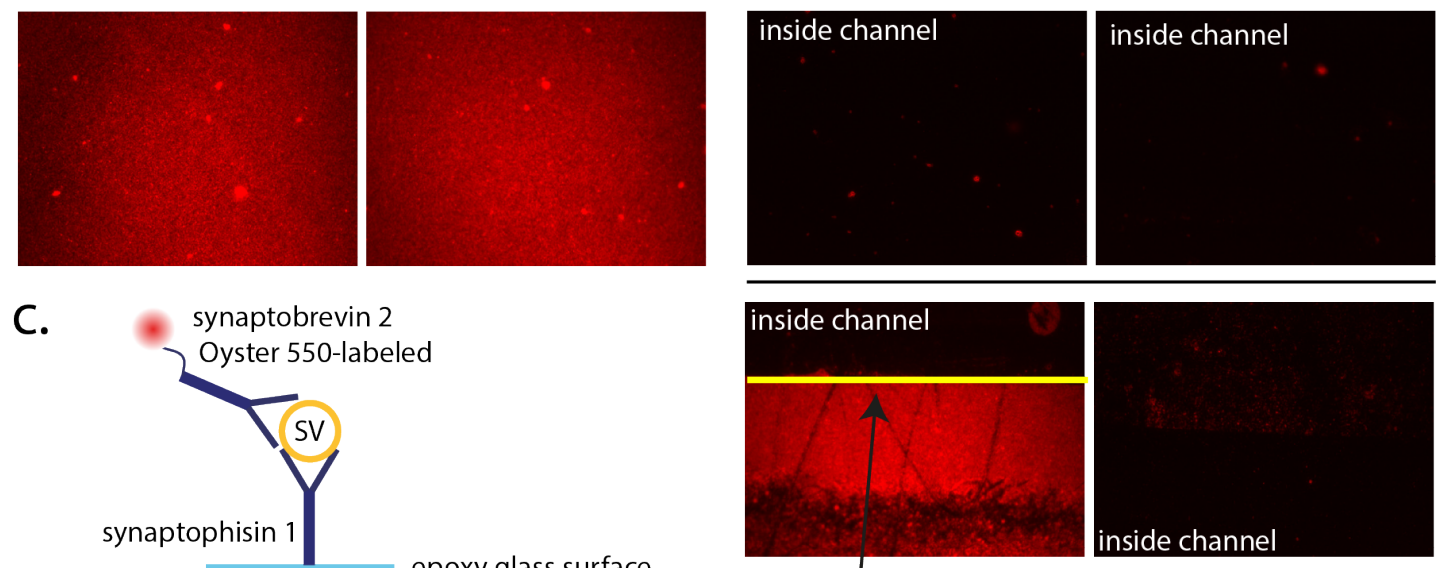

epoxy glass surface

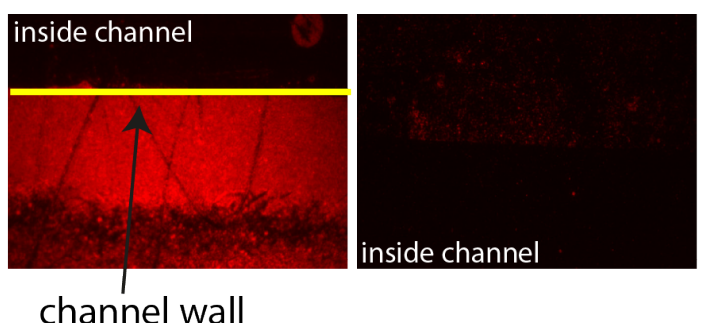

Figure 4.41: (b) Epi-fluorescence images of the SV patterns after 30 minutes in the microfluidic device with PBS flowing on the pattern at a velocity of $4 \mathrm{~mm} / \mathrm{s}$. After 30 minutes of flow, the vesicles are visualized with a synaptobrevin antibody labeled with Oyster-550, as shown in the sketch. (a) To check if there are differences when no flow is applied on the vesicles, the coverslip with the SV pattern are not mounted in the device holder and no flow is applied onto the vesicles. After 30 minutes of flow, the vesicles are detached from the glass surface. Two different patterns, from different coverslip are shown respectively in the two lines. The images were acquired by Helena Maria (Linda) Olsthoorn. 
a.

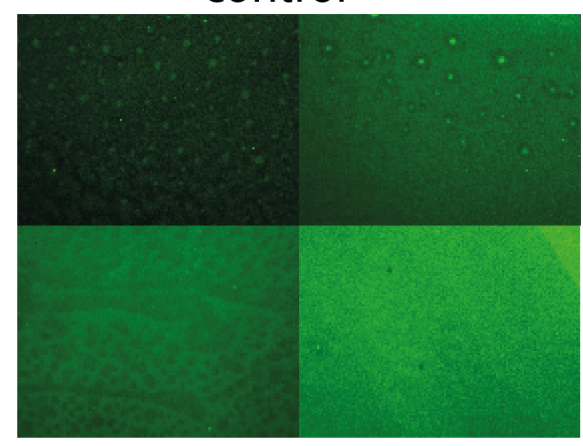

C.

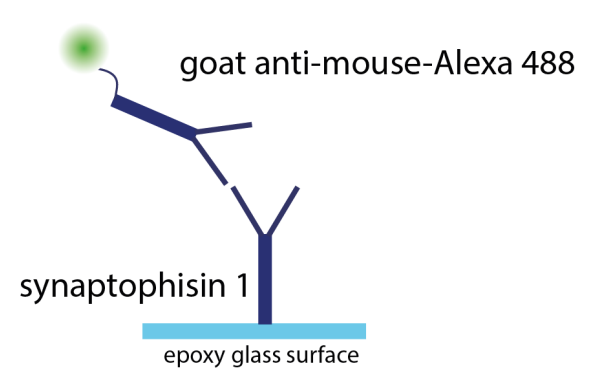

b.

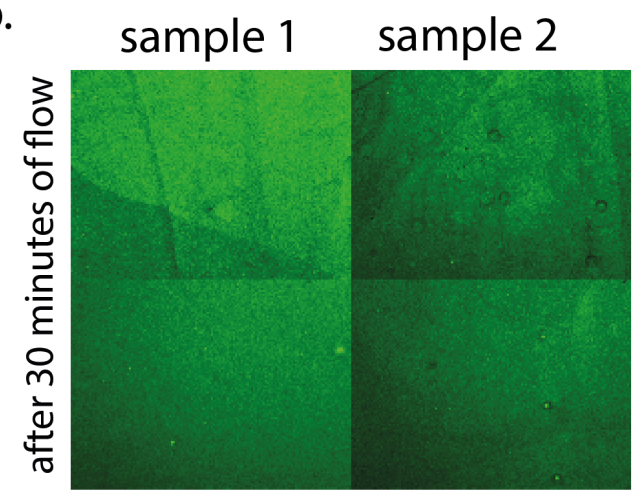

d.

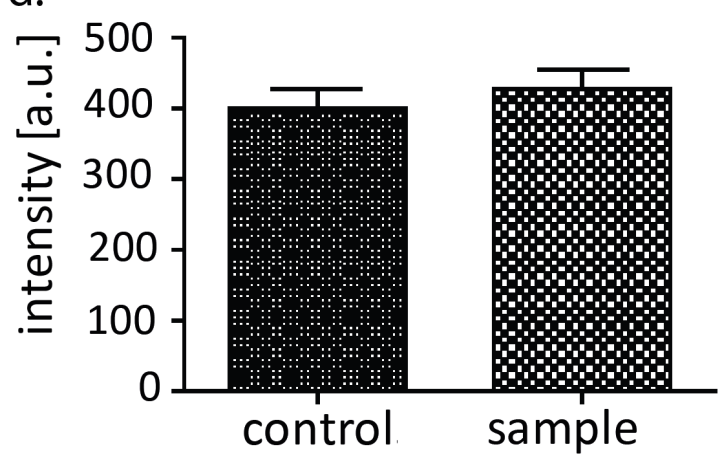

Figure 4.42: Epi-fluorescence images of the antibody patterns before (a) and after (b) 30 minutes in the microfluidic device with PBS flowing on the pattern at a velocity of $4 \mathrm{~mm} / \mathrm{s}$. (c) After 30 minutes of flow, the antibodies are visualized with a goat $\alpha$-mouse antibody labeled with Alexa-532. The controls were mounted in the device holder, but no flow was applied to the patterned antibodies. Two different samples with synaptophisin1 are tested in the microfluidic device. (d) The images show some variability in the fluorescence signal, however there is no significant difference between the average fluorescence intensities between the controls and the samples. The images were acquired by Helena Maria (Linda) Olsthoorn.

device. The epi-fluorescence images show some variability in the fluorescence signal, however there is no significant difference between the average fluorescence intensities between the controls and the samples. Thus, it would be interesting to study the attachment of the SV to the patterned antibody at different flow rates to quantify a critical value for the flow rate.

A more promising approach is now employed to covalently attach antibodies to the glass surface. This method is introduced in the paper from Cretich et al. [155], where a polymer coating is used to allow high density of native proteins to the substrate. The first preliminary data, not shown here, are promising. No sign of SVs detachment is visible under flow and soon it will be possible to study $\mathrm{SVs}$ reactions, as SVs acidification, with high temporal resolution as never done before. 


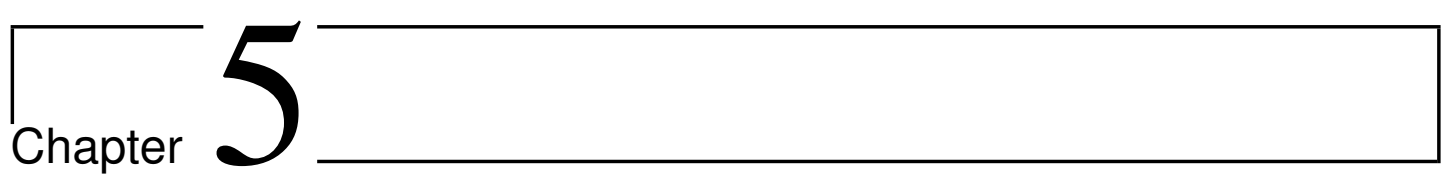

\section{Summary, conclusions and outlook}

Protein assembly and molecular interactions are fundamental processes in living organisms. In some cases, protein assembly is vital for physiological cell behavior, such as the formation of the cytoskeleton, hemoglobin assembly in red blood cells or the spindle creation during mitosis. In other cases, disordered aggregates of proteins can be toxic for the organism. Usually, the body can detect and discard the products of these faulty assembly reactions but, sometimes, the aggregates can go unnoticed and lead to pathological situations 22. Some diseases that are related to unsoluble aggregate formation are not life threatening, such as cataracts [3], some, on the contrary, are more severe, such as the synucleinopathies [4].

FFS can be used to measure protein aggregation both in vivo [11, 20, 40] and in vitro [35, 10, 12, 132. FFS is a family of techniques based on the analysis of the intensity fluctuations over time to obtain parameters such as the diffusion coefficient and the brightness or the concentration of the single fluorophores crossing the observation volume. To properly acquire the fluctuations, low sample concentrations and a well-defined volume of observation are fundamental requirements [6]. In this thesis, we applied FCS and PCH to measure interactions and assembly of proteins. In FCS, the fluctuation intensities are correlated in time to gain information about protein mobility and diffusion [17. This technique is dependent of the fluctuation duration and density, but it is independent of the laser power applied. Everything that might interfere with the fluctuation duration, for example applied convective flow on the sample, changes the analytical form of the correlation function. At low flow velocities, FCS can still be applied to measure diffusion, but if the flow velocity is too high, the molecular movement is governed by convective flow and not diffusion [27]. Complementary to FCS, PCH is not dependent on the flow velocity [53], but it is dependent on the laser power and the optical path of the setup. With $\mathrm{PCH}$, instead of analyzing the fluorescence fluctuations in time, a histogram of the intensities is built to gain information about the brightness and the concentration of the molecules in the focal volume [8]. It can be employed, for example, to measure the stoichiometry of molecules 
during aggregation [39, 53] or to measure protein interactions at a single molecule level [62]. A disadvantage of these techniques is the long acquisition time which is needed to obtain a good signal-to-noise ratio. For a single FCS curve, typically $10 \mathrm{~s}$ of acquisition time is sufficient, while for $\mathrm{PCH}$ longer acquisition times of around $800 \mathrm{~s}$ are needed. Thus, fast dynamics cannot be measured in bulk.

However, it is fundamental to study protein assembly and interaction with a good temporal resolution. In fact, a complete knowledge of the dynamics of pathological protein aggregation might help to prevent the anomalous steps during the protein interactions which might lead to diseases. To access the temporal information, microfluidics is employed during the experiments. Microfluidic techniques are largely applied in biophysics since they can be used as a tool to reliably control small scale systems [13. In particular, protein assembly and molecular interactions are studied here under continuous flow microfluidics, which allows for tracking the progress of the reaction over time, as already shown in literature [14, 15, 16. In our experiments microfluidic devices also work as mixers [163]. The protein jet is focused to a small stream prior to the mixing, to achieve control and fast mixing [75].

An example of the combination of microfluidics and FFS, in particular PCH, is the first project of this thesis, presented in Section 4.1, where the early time points of vimentin assembly are tracked over time. A five-inlet step microfluidic device compatible with microscopy and FFS is manufactured. The adsorption of assembling vimentin on the channel walls is avoided by creating a step in the central channel, which helps to encapsulate the vimentin jet into a buffer protection layer and prevents the protein from touching the device walls [14]. FEM simulations are performed of the conditions in the microfluidic device to optimize the geometry and the flow rates for our measurements. The independence of $\mathrm{PCH}$ from convective flow is experimentally tested on vimentin tetramers in a single channel, confirming the results obtained by Johnson et al. 553. PCH measurements of vimentin tetramers in bulk and in flow are used as a baseline for the assembly experiments. The protein assembly is initiated by diffusion mixing vimentin tetramers and assembly buffer. Once the assembly is started, PCH measurements are acquired at different positions along the outlet channel, thus for different time points of the assembly reaction. When vimentin tetramers are mixed with the assembly buffer, the determined brightness increases over time. The brightnesses can be also predicted from the binomial distribution (equation 4.1) once the initial labeling ratio of vimentin molecules is known. The brightness measured at $t=0 \mathrm{~s}$ corresponds to the value calculated with equation 4.1, while the final brightness value measured corresponds to the ULF calculated value. Thus, the lateral vimentin assembly can be measured with our technique. The brightness curves over time are fitted with an exponential growth function to evaluate the time needed for vimentin lateral assembly. With this method, we are able to quantify that vimentin lateral assembly is a very rapid process, and on average the reaction time for the formation of ULF at our conditions is $65 \mathrm{~ms}$. 
The method presented here allows us to directly evaluate the very first time points of vimentin assembly, which are difficult to measure with static techniques, such as AFM [89, 91], time-lapse EM [119, 88] or light microscopy [114. Light scattering techniques, such as SLS and time-resolved multi-angle DLS, were applied in bulk to quantify the early elongation process of vimentin filaments [87, 94 . However, with light scattering in bulk the formation of ULFs cannot be quantified, since the time needed to mix vimentin tetramers with the assembly buffer is actually longer than the time scales for vimentin lateral assembly. The absence of a tool to quickly mix the assembly buffer with vimentin just prior the acquisition of the data is the main reason for the lack of the early time scales of assembly in these scattering experiments. Thus, microfluidics is an essential tool which allows us to quickly mix vimentin and assembly buffer and start measuring vimentin assembly without any time delay. Our measurements are able to evaluate more precisely the lateral time scale of vimentin assembly, which was measured to be within one second with a combination of SAXS and microfluidics [14, 15, 134. Moreover, the diffusion mixing achieved with microfluidics allows for a "gentle" and controlled mixing, which is close to the dialysis vimentin assembly performed in vitro. When vimentin tetramers are mixed with less controlled methods, such as stopped-flow mixing, some variability in the average number of monomers per vimentin assemblies is measured [96]. In the paper by Mücke et al. [96], stoppedflow mixing is combined with SLS, allowing the authors to measure the early time points of vimentin assembly with high temporal resolution. Our measurements confirm the time scales for vimentin lateral assembly measured in Mücke et al., where a time scale within $100 \mathrm{~ms}$ is measured for the formation of ULFs.

In the second part of the thesis, in Section 4.2, FFS and microfluidics are applied to study molecular interactions in the synapse. Synapses are very crowded environments [99], thus measuring molecular interactions in vivo is challenging. A bottom up approach is chosen to study synaptic interaction in a more controlled environment, in particular a simpler version of the synaptic environment is reconstituted on a substrate by patterning SVs on glass coverslips. The vesicle patterns are used to measure the interaction between $\alpha$-synuclein and SVs employing FCS. $\alpha$-synuclein has two diffusing behaviors when incubated on the SVs. A first component which can be related to the freely diffusing protein is measured, however, the diffusion coefficient obtained for this component is lower than expected from bulk measurements possibly due to crowding caused by the pattern. The crowding effect could be tested by varying the density of the SVs on the pattern. The second component with a smaller diffusion coefficient is compatible with $\alpha$-synuclein interacting with the vesicles. Nevertheless, the diffusion coefficients show some variability which could be correlated with the different structural conformations of $\alpha$-synuclein [5]. Similar crowding effects are also detected when the positive and the negative antibodies are measured with FCS on top of the vesicle patterns. An additional diffusion coefficient, smaller than the one for the free diffusion component, is obtained for the positive antibody, which 
corresponds to the interacting antibodies with the SVs. These results are the proof of principle that SVs can be patterned on glass coverslips and they can be employed to measure the interaction between SVs and synaptic proteins in a controlled environment. Since the goal of the project is to create a map of the interactions between SVs and synaptic proteins, more proteins will have to be measured.

To access the temporal information of the synaptic reactions a microfluidic chamber is manufactured to study SV interactions under flow with a temporal resolution on the time scales of ms. Even if some SV pathways are well studied [110, the kinetics of vesicle acidification and neurotransmitters uptake [154] still remain elusive. Thus, microfluidics helps here, as in the first project, to access the time points of the reaction in a controlled environment. The bottom surface of the microfluidic chambers is constituted by a glass coverslips with a pattern of SV in the center of the outlet channel. To enable us to seal the microfluidic device a custom device holder, which is inspired by [118, 134, composed by two metal plates clamped together, is employed to prevent any leaking. The flow controllability, a typical advantage of microfluidics, is exploited here. The fluids are controlled using a rectangular velocity profile, allowing us to quickly switch the solution flowing on top of the SV pattern in the center of the outlet channel. Microscopy and FEM simulations are employed to test how fast we can switch the solution flowing on top of the SVs in the microfluidic device. The reaction time in the device to switch the solution depends on the geometry of the device; for a device with a width of $500 \mu \mathrm{m}$ we are able to switch the solution in $250 \mathrm{~ms}$, for a bigger device (width $=1 \mathrm{~mm}$ ) the reaction time is higher $(460 \mathrm{~ms})$ but still below $1 \mathrm{~s}$. Thus, the fast reaction time will allow us to study synaptic reactions with a very short dead-time. However, the strength of the patterned SVs has to be improved to be able to exploit the system in actual experiments. A new promising method, based on a polymer coating [155] which increases the density and the strength of the covalent link between the surface and the SVs, is going to be tested soon in our microfluidic chamber.

Since now, only the in vitro synaptic interactions are described, with and without temporal information, however we still want to compare these "simpler" experiments with measurements in living cells. Consequently we applied FCS also on living neurons to measure protein mobility. However, some difficulties are encountered during the analysis of these data. Pronounced photobleaching is an important factor, especially at high concentrations of the expressed proteins [36], which reduces the quality of the acquired data. Moreover, usually, in FCS, a prior knowledge of the system to study is needed to properly analyze the data [6] and it was shown that possibly the FCS analysis is different for different cells line [137]. In this case, not many experiments with similar conditions on living neurons are available to compare the results. Nevertheless, different FCS models, as for example, models accounting for the cell geometry or caged diffusion [149, 165], might be tested, under the supervision of statistic tools as the Bayesian 
information criterion [166].

To conclude, in this thesis three main results are achieved. First, a microfluidic device to study protein interactions and assembly with FFS is presented. The combination between FFS and microfluidics allows us to study reaction dynamics with high temporal resolution and spatial resolution at a single molecule level. Furthermore, microfluidics helps to decrease the dead-times during mixing, making the very first temporal steps of protein assembly accessible. Second, early time points of vimentin assembly are measured combining microfluidics and PCH. Vimentin lateral assembly is quantified and compared with the current state of the art. Our measurements of vimentin lateral assembly can be used to fill the gap of the early time points in the overall dynamics of vimentin. Third, we show that the interactions between synaptic proteins and SV can be measured in vitro on patterned glass substrates. In particular, the interaction of $\alpha$-synuclein and SVs is measured on top of SV patterns using FCS. A microfluidic chamber is built, compatible with patterned molecules, to allow us to access also the temporal information during synaptic interactions.

We showed that the combination of microfluidics and FFS techniques can be applied in general to study the dynamic of various processes in biology, varying from cytoskeleton assembly to synaptic protein interactions. $\mathrm{PCH}$ and the step microfluidic device can also be employed to study the assembly of other IFs, such as keratin or desmin. In particular, keratin has a much faster assembly time compared to vimentin [167], thus it is even more difficult without microfluidics to access the very first time points during the assembly. Regarding desmin, in some pathogenic mutations of this IF, aggregates might be formed instead of proper filaments, causing progressive weakness in the skeletal muscles and cardiomyopathies 161. In some desmin mutations, it was discovered that some of the first disruptions of the assembly pathway happen during the dimer-dimer interaction [160]. The aggregate formation of mutant desmin can be studied and characterized deeper with our technique, which will allow us to measure at a single molecule level, where and how the assembly went wrong. The combination of microfluidics and FFS presented here can be easily applied also on other biological systems, as for example proteins in the synuclein family. Conformational changes, aggregation and fibril formation might be measured directly in the microfluidic device, monitoring over time the proteins under the different buffer conditions. Similar experiments, can be performed using synaptic proteins and SVs to measure the dynamics of the interactions between the two components. 


\section{Ringraziamenti /Acknowledgement}

Finally, the time to acknowledge everyone is arrived. First of all, my biggest thanks goes to my thesis supervisor Prof. Sarah Köster, who helped me and inspired me during all my PhD. Thanks for the opportunity of working at this project, and thanks for all the support, the positivity and the constructive meetings during these years. Thanks to Prof. Silvio Rizzoli, for being my cosupervisor, for co-refereeing this thesis, for all the comments as a member of my thesis committee and for all the cool suggestions during the meetings. I want also thank also Prof. Christian Griesinger for being a member of the thesis committee and for taking part of the examination board. Thanks also to Prof. Detlev Schild for all the advises when he was part of my thesis committee. I am grateful to Dr. Sebastian Kruss, Dr. Sarah Adio and Prof. Helmut Grubmüller for agreeing to be a part of the examination board. Thanks also to Prof. Erwin Neher, who had the inspiration for part of this work. Thanks to Prof. Harald Herrmann and Dr. Norbert Müucke for providing the vimentin plasmids and for sharing their expertise during the IF meetings. A huge thanks to our collaborators Linda and Prof. Reinhard Jahn for the project of the vesicles under flow, and to Sofiia and Prof. Silvio Rizzoli for the experiments on living neurons and on SVs. I want also to express my gratitude to Prof. Don Lamb and Prof. Enrico Gratton for helping me understand the PCH theory.

I want to thank Prof. Tim Salditt and all people from the IRP for all the valuable discussions during the seminars or in front of a cake slice. I express my gratitude to all the IRP secretaries, Kerstin, Sabine, Michaela and Christine for taking care of all the administrative formalities. Also, thanks to Jan Goeman for the IT technical assistance and to Peter Luley and Peter Nieschalk for the help solving technical problems. Thanks to Jochen Herbst for the clean-room assistance. A big thanks to Ulrike, Kamila and Susanne for all the help in the labs.

Thanks to Susanne Bauch (my german adoptive mum) for preparing vimentin, for the help in the labs and for all the day-to-day nice conversations in the office. An immense thanks to all the group members! To Manuela for having 
shared with me this crazy journey called PhD, to (mama) Julia for having feed all of us with amazing cakes (and Christmas cookies), to Lotta for being always a super positive and smiling person, to Anna for having me taught how to use the sewing machine and for all the crazy conversations on the way to the mensa, to Heidi for being a little bit crazy in a very cute way, to Gerrit for being always there in the right moment, to Anička for being an amazing part of the ACE (the coolest people on the planet!), to Andrew for all the prepared coffee and the lost bets, to Jan Philipp for his patience, Anna (Nr. 3) for always talking in english when the italian crew is around and a really super huge thank to Chiara for being a truly really wonderful friend. Thanks to Charlotte, Laura, Julia B. and Magdalena for creating a wonderful working environment. I want to thank Sebastian Smyk for the amazing work during his bachelor thesis. I also thank all the former group members: Aishwarya, Jana, Johanna, Oliva, Clément, Viktor for sharing expertises and providing a nice atmosphere in the group. More important, again a huge thanks to all of you for having helped me during these years several times; all of you are super wonderful people, and I am really lucky and grateful to have met you. Thanks to the GGNB and IMPRS Physics of Biological and Complex Systems, for providing a good structure at the graduate school. And an acknowledgement goes to also the German Research Foundation for funding the projects in the framework of the SFB 1286 Quantitative Synaptology.

A big thank goes also to everyone who supported me from Italy during these years. Forse il piu' importante ringraziamento va ai miei genitori, Elisabetta e Dino, che mi hanno davvero sempre supportato e sopportato in questi anni. Questo periodo non è sempre stato facile, ma insieme lo stiamo superando, grazie davvero di cuore. Siete il mio punto di riferimento ed il mio porto sicuro da cui partire per nuove avventure. Grazie a mia cugina Marta, che è sempre una pazza ma che ogni tanto ha ragione quando dice che mi manca (ma anche io ti manco R.W.!). Grazie a mio zio Antonio e a Daniela, i vostri mega abbracci quando ci vediamo scaldano sempre l'anima. Grazie mille anche a Sabrina, nonostante non ci vediamo propeio spesso, ogni volta che succede e' come se io fossi sempre li, ed e' proprio questo il bello di essere una grande famiglia. Grazie ai miei nonni Dorina e Mario che mi hanno insegnato l'importanza della memoria e dei ricordi. Un mega grazie a Roberto e Mariangela ed hai miei cugini Elena e Marco. Di nuovo, un grazie enorme a tutta la mia famiglia. Nonostante non abbiano capito di preciso cosa io stia facendo in Germania, non hanno mai smesso di incoraggiarmi ed avere fiducia in me. Un grazie davvero speciale va ai miei nonni Gianfranco e Rosangela che anche se non ci sono più, mi hanno sempre supportato con tutto il cuore e sono sicura che sarebbero stati super orgogliosi della loro dottoressa.

Un grazie speciale va a due miei amici di vecchissima data, Francesco e Liliana. Ci conosciamo da quasi 25 anni, siamo cresciuti insieme e non ci siamo mai 
lasciati. Grazie a Fra che con le tue foto mi hai rallegrato ogni giornata grigia. E grazie a te Lilly, non esiste distanza troppo grande che noi non possiamo superare, in qualunque posto saremo, rideremo sempre e sempre insieme come sappiamo fare noi. Un grazie immenso a tutti i Flower Power! E' fanstastico tornare a casa ed ogni volta sentire che si è davvero a casa quando siamo tutti insieme. Siamo come una seconda famiglia, siamo cresciuti nel tempo cosi' come sono cresciuti i rapporti che ci legano. Siamo davvero bellissimi tutti insieme! Per cui grazie di cuore a tutti voi, Andrea, Claudia, Chiara, Christian, Daniele, Erika, Francesca, Francesco, Giulia, Lodovico, Samuele, Serena e Silvia.

Probabilmente (anzi sicuramente) avro' dimenticato qualcuno, pero' vorrei ringraziare di nuovo tutte le persone che ho incontrato durante il mio percorso; e' grazie a voi se sono diventata la persona che sono oggi. E' grazie a voi se sono arrivata fino a qui. 


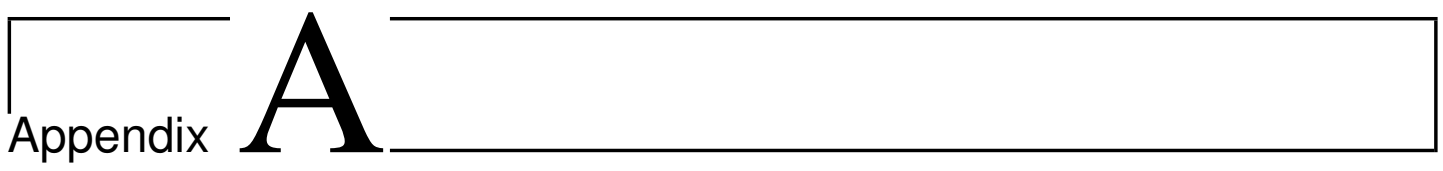

\section{Appendix}

\section{A.1 Microcontact printing}

Microcontact printing ( $\mu$-CP) is a soft-lithography method used to transfer ink to surfaces starting from a PDMS stamp. In this thesis $\mu-\mathrm{CP}$ was employed before the installation of the PRIMO patterning system to create the SV patterns (described in Section 3.2). Both with PRIMO and $\mu$-CP, the patterned molecules are adsorbed on the surface, thus there are no differences in the obtained patterns between the two methods. Neutravidin is printed on glass coverslips allowing, later, the attachment of SVs. The method described below is adapted from [168].

PDMS stamps are produced mixing the liquid PDMS with the cross-linker at a ratio of $10: 1$ and the liquid mixture is poured on top the master silicon wafer. The liquid PDMS is cured in the oven at $65{ }^{\circ} \mathrm{C}$ for 1 hour after the removal of the air-bubbles in a desiccator. The master wafers were produced by photo-lithography by a previous PhD student, Dr. Rabea Sandmann. A sketch of the main steps of the $\mu$-CP method applied on glass coverslips is shown in Figure A.1. Neutravidin with a concentration of $0.05 \mathrm{~g} / \mathrm{L}$ is incubated on top of the PDMS stamps for 1 hour (Figure A.1 a). Shortly before the incubation time is over, the glass coverslips are washed in a sonicator with Isopropanol for 5 minutes. The glass substrates are activated using plasma cleaning (Plasma Cleaning, PDC-32 G, Harrick Plasma) at level "medium" for 1.5 minutes. Then, the neutravidin solution is removed and the stamps are washed twice, each time for about 5 seconds, in fresh ultrapure water. The stamps are dried using a dry nitrogen gun. The neutravidin solution is now loaded on top of the PDMS stamps (Figure A.1 c). The stamps are placed on top of the activated glass coverslips (Figure A.1 c) for 15 minutes. The neutravidin is transferred from the PDMS stamp to the glass coverslip, leaving the substrate with a protein pattern. An epifluorescence image of printed neutravindin-FITC is shown in Figure A.1 e. To prevent unspecific binding, the surface is incubated with a solution of Poloxamer 407 (Sigma-Aldrich, St. Louis, Missouri, USA) at 0.2\%, at room temperature for 
a. protein solution

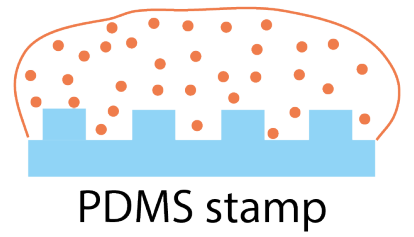

C.

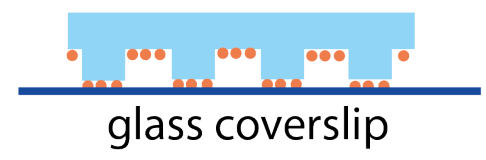

b.

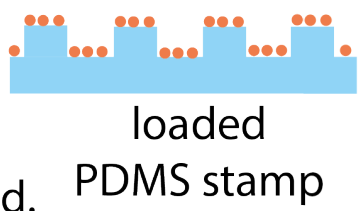

e.

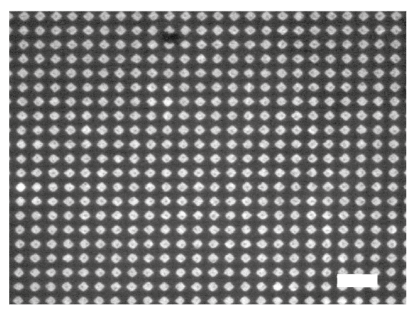

Figure A.1: Schematic representation of $\mu$-CP. Once the PDMS stamps are produced from the master wafers, (a) the protein is incubated on top of the PDMS stamps for 1 hour. (b) The stamps are washed in ultrapure water to remove the excess of protein. (c) After the activation of the glass substrates by plasma treatment, the PDMS stamps are pressed on the glass coverslip for 15 minutes. (d) The adsorbed protein on the PDMS stamps is transferred to the glass coverslips. (e) Epi-fluorescence image of printed neutravidin-FITC $(c=0.05 \mathrm{~g} / \mathrm{L})$. The scale bar is $10 \mu \mathrm{m}$.

2 hours. Afterwards, the passivating solution is removed and the printed glass coverslips are washed 3 times with PBS before continuing with the protocol to attach SVs.

\section{A.2 Detailed FCS derivation}

FCS represents a family of correlation methods used to describe fluorescence behaviour. Here, there will be a step-wise mathematical derivation of the ACF (auto-correlation function), where the intensity signal is correlated with itself, for identical molecules diffusing in a confined volume. The normalized ACF can be defined as follows:

$$
G(\tau)=\frac{\langle\delta F(t) \cdot \delta F(t+\tau)\rangle}{\langle F(t)\rangle^{2}}
$$

where $\delta F(t)$ represents the fluctuation of fluorescence intensity in a certain time $t$ measured on the detector, and $\tau$ is the time delay relative to an earlier time point in the measurement. The fluctuations of the fluorescence intensity measured by the detector can be written as:

$$
\delta F(t)=k \int_{V} I_{e x c}(\vec{r}) S(\vec{r}) \delta\left(\sigma_{a b s} \Phi C(\vec{r}, t)\right) d V,
$$

Here, $S(\vec{r})$ is the optical transfer function of the objective-pinhole, $V$ is the volume, $C$ is the concentration of the diffusing fluorophores, $\sigma_{a b s}$ is the cross 
section for absorption, $k$ is the quantum efficiency of photon detection and $\Phi$ is the quantum yield of emission. This equation can be simplified, introducing the spatial distribution of the emitted light on the detector plane $W(\vec{r})$, defined as $W(\vec{r})=I_{e x c}(\vec{r}) S(\vec{r})$, and the molecular brightness $b$, defined as $b=k \sigma_{a b s} \Phi$. Thus, equation A.2 can be written as:

$$
\delta F(t)=\int_{V} W(\vec{r}) \delta(b C(\vec{r}, t)) d V,
$$

The explicit form of the fluorescence fluctuations can be inserted into Equation A.1.

$$
G(\tau) \frac{\iint_{V V^{\prime}} W(\vec{r}) W\left(\overrightarrow{r^{\prime}}\right)\left\langle\delta(b C(\vec{r}, t)) \delta\left(b C\left(\overrightarrow{r^{\prime}}, t+\tau\right)\right)\right\rangle d V d V^{\prime}}{\left(\int_{V} W(\vec{r})\langle\delta(b C(\vec{r}, t))\rangle d v\right)^{2}} .
$$

The fluctuations of the fluorescence signal are dependent on the concentration of the diffusing fluorophores and on the molecular brightness:

$$
\delta(b C(t))=b \cdot \delta C(t)+\delta b \cdot C(t),
$$

However, if a constant molecular brightness is considered $(\delta b=0)$, only the concentration component is relevant for the fluorescence fluctuations:

$$
\delta(b C(t))=b \cdot \delta C(t),
$$

This is true when there are no photo-physical effects. In this case equation (A.4) can be written as:

$$
G(\tau)=\frac{\iint_{V V^{\prime}} W(\vec{r}) W\left(\overrightarrow{r^{\prime}}\right)\left\langle\delta C(\vec{r}, 0) \delta C\left(\overrightarrow{r^{\prime}}, \tau\right)\right\rangle d V d V^{\prime}}{\left(\int_{V} \delta C(\vec{r}, 0) W(\vec{r}) d v\right)^{2}},
$$

where $t=0$ is defined for simplicity. The correlation function for molecule moving with Brownian motion in a three dimensional space is given by:

$$
\left\langle\delta C(\vec{r}, 0) \delta C\left(\overrightarrow{r^{\prime}}, \tau\right)\right\rangle=\langle C\rangle \frac{1}{(4 \pi \tau D)^{3 / 2}} e^{\left(\vec{r}-\vec{r}^{\prime}\right)^{2} / 4 D \tau},
$$

where $D$ is the diffusion coefficient. Then, it is possible to re-write equation (A.7) as:

$$
G(\tau)=\frac{1}{\langle C\rangle(4 \pi \tau D)^{3 / 2}} \frac{\iint_{V V^{\prime}} W(\vec{r}) W\left(\overrightarrow{r^{\prime}}\right) e^{\left(\vec{r}-\vec{r}^{\prime}\right)^{2} / 4 D \tau} d V d V^{\prime}}{\left(\int_{V} W(\vec{r}) d v\right)^{2}}
$$

In the confocal case, $W(\vec{r})$ is described by a 3-D Gaussian:

$$
W(\vec{r})=I_{0} e^{-2\left(x^{2}+y^{2}\right) / w_{0}^{2}} e^{-2 z^{2} / z_{0}^{2}},
$$


where $\vec{r}=(x, y, z)$, and $w_{0}$ and $z_{0}$ are the beam profile parameters.

To simplify equation A.9, the effective volume $V_{\text {eff }}$ can be defined as:

$$
V_{e f f}=\frac{\left(\int_{V} W(\vec{r}) d V\right)^{2}}{\int_{V} W(\vec{r})^{2} d V}
$$

For the confocal case, $V_{\text {eff }}$ is equal to $V_{\text {eff }}=\left(\frac{\pi}{2}\right)^{3 / 2} w_{0}^{2} z_{0}$. Evaluating the volume integrals in A.9, we can explicitly write the ACF for diffusing molecules in the effective volume defined by the PSF:

$$
G_{D}(\tau)=\frac{\gamma}{\langle C\rangle V_{e f f}} \frac{1}{1+\frac{\tau}{\tau_{D}}} \frac{1}{\sqrt{1+\frac{\tau}{\tau_{D}}\left(\frac{w_{0}}{z_{0}}\right)^{2}}},
$$

For the one photon excitation (OPE) case $\gamma=0.35$ ( $\gamma$ is a constant related to the PSF calculation, $1 / \sqrt{8})$ and $\tau_{D}=\frac{w_{0}^{2}}{4 D}$.

The average number of molecule in the effective volume can be calculated from the average concentration:

$$
N=\langle C\rangle V_{e f f},
$$

Usually, the ACF function is written as:

$$
G_{D}(\tau)=G(0) \frac{1}{1+\frac{\tau}{\tau_{D}}} \frac{1}{\sqrt{1+\frac{\tau}{\tau_{D}}\left(\frac{w_{0}}{z_{0}}\right)^{2}}}
$$

where the amplitude of the $\mathrm{ACF} G(0)$ is defined as:

$$
G(0)=\frac{\gamma}{\langle C\rangle V_{e f f}}
$$

The extrapolation of the $\mathrm{ACF}$ at 0 lag time $(G(0))$ is inversely proportional to the fluorophore concentration.

\section{A.3 Detailed derivation of the photon count distribution}

A detailed derivation of the $\mathrm{PCH}$ is described in the following section. All the derivation can be found also in [8]. The analytical description of the PCH starts from the distribution of intensities measured by the detector:

$$
p(k, t, T)=\int_{0}^{\infty} \frac{\left(\eta_{W} W(t)\right)^{k} e^{-\eta_{W} W(t)}}{k !} p(W(t)) d W(t),
$$

This is Mandel's formula [46], which describes the photon counting statistic measured by the detector for the semiclassical cases. In the description of the photon 
statistics the semiclassical approximation is often applied since it allows us to describe the light using the electromagnetic classical theory while the interaction with atoms is described with quantum mechanics. $p(k, t, T)$ is the probability of observing $k$ photoelectrons at time $t$ on the detector and it depends on the detection efficiency $\eta_{W}$, the integration time $T$ and the energy distribution $p(W(t))$ on the detector. $W(t)$ represents the light energy falling on the detector surface during one integration time $T$ and it can be described by:

$$
W(t)=\int_{t}^{t+T} d t \int_{A} I_{D}(\vec{r}, t) d A
$$

where $A$ is the detector area and $I_{D}(\vec{r}, t)$ is the light intensity reaching the detector at time $t$. From a mathematical point of view equation A.16 is a double stochastic Poisson process based on two sources of randomness. The first source of randomness is a quantum mechanical effect and it is a manifestation of the discreteness and the statistical independence of the photoelectric detection process for coherent electromagnetic radiation. It is known as shot noise and it cannot be eliminated. Even if the source of the light intensity is constant, thus described as $p(W)=\delta(W-\bar{W})$, the photon count distribution is still represented by a Poissonian distribution:

$$
p(k, t, T)=\operatorname{Poi}(k,\langle k\rangle)=\frac{\left(\eta_{W} \bar{W}\right)^{k} e^{-\eta_{W} \bar{W}}}{k !} .
$$

In this case, since the distribution is Poissonian, the variance of the distribution $\left\langle\Delta k^{2}\right\rangle$ is equal to the average of the distribution $\langle k\rangle$.

The second source of randomness is caused by the fluctuations of light intensity on the detector, which is described by $p(W)$. Any source of noise will cause an additional broadening of the photon count distribution $p(k, t, T)$. The photon count distribution is now described by a super-Poissonian distribution, where the variance is larger then the mean value $\left.\left(\left\langle\Delta k^{2}\right\rangle\right\rangle\langle k\rangle\right)$. Actually, equation A.16 can be considered as a superposition of Poissonian distributions for each of the energy values $W$, with the amplitudes given by of $p(W)$. The broadening of the photon count distribution is dependent on $I_{D}(\vec{r}, t)$, which is dependent on the integration time $T$. In the limit of $T \rightarrow \infty$ the fluctuations average out, $p(W)$ approaches a delta function and the photon count distribution narrows down to a Poissonian distribution, because the shot noise contribution still remains. In the other limit $T \rightarrow 0$, the energy fluctuations $W(t)$ perfectly tracks the light intensity $I_{D}$ in time. Thus, the probability distribution of energy and intensity are proportional to each other $p(W)=p(I) \Delta t$. For simplicity, the detector area $A$ is chosen to be small enough, so that the intensity $I$ is constant across the detector surface during one binning time $T$. To properly capture the intensity fluctuations in the photon count distribution, the integration time $T$ has to be chosen shorter than the fluctuation time scales of the studied process $\left(T<\tau_{\text {fluc }}\right)$. We will assume this condition to be valid for all the following derivation. We are 
also assuming that the fluctuations of intensity are a stationary event, i.e. there is no time dependence to the statistical properties of the fluctuating light intensity $I$ and therefore no time dependence to the $\mathrm{PCH}$. The detection efficiency $\eta_{W}$ can be rewritten proportionally to the light intensity as:

$$
\eta_{I}=T \eta_{W} .
$$

In this case we can rewrite equation A.16 as:

$$
\begin{gathered}
p(k, t, T)=\int_{0}^{\infty} \frac{\left(\frac{\eta_{I}}{T} I_{D} T\right)^{k} e^{-\frac{\eta_{I}}{T} I_{D} T}}{k !} p\left(I_{D}\right) d I_{D}, \\
p(k, t, T)=\int_{0}^{\infty} \frac{\left(\eta_{I} I_{D}\right)^{k} e^{-\eta_{I} I_{D}}}{k !} p\left(I_{D}\right) d I_{D},
\end{gathered}
$$

This equation is actually describing a Poissonian distribution:

$$
p(k, t, T)=\int_{0}^{\infty} \operatorname{Poi}\left(k, \eta_{I} I_{D}\right) p\left(I_{D}\right) d I_{D},
$$

At this point, we have to describe the fluorescence intensity. The confined excitation volume generated by the microscope allows us to measure the fluorescence intensities. This volume is dependent on the PSF of the system, which describes in general the response of an optical setup to a point source. In this derivation context it is convenient to define a scaled PSF, which is normalized to the origin:

$$
\overline{P S F}=\frac{P S F(x, y, z)}{P S F(0,0,0)},
$$

For our experiments (confocal case) the PSF is approximated by a 3D Gaussian:

$$
\overline{P S F}_{3 D G}=\exp \left[-\frac{2\left(x^{2}+y^{2}\right)}{w_{0}}-\frac{z^{2}}{z_{0}}\right],
$$

where $w_{0}$ and $z_{0}$ are the effective parameters that describe the PSF geometry. The fluorescence intensity $I_{D}$ at the detector plane, which is emitted by a single fluorophore at a position $\overrightarrow{r_{0}}$ (defined as the PSF center), can be written as:

$$
I_{D}=I_{0}^{n} \beta \overline{P S F}\left(\overrightarrow{r_{0}}\right)
$$

For the confocal case $n=1$. The constant $\beta$ includes the excitation probability, the fluorescence quantum yield and all the set-up related factor such as the transmittance of light after the optics.

We can use equation A.24 to describe the probability of fluorescence intensity on the detector $p\left(I_{D}\right)$ which is in the equation A.21.

$$
p\left(I_{D}\right)=\int \delta\left(I_{D}-I_{0} \beta \overline{P S F}(\vec{r})\right) p(\vec{r}) d \vec{r},
$$


Here $p(\vec{r})$ is the probability distribution for the position of the fluorophore. Thus, the photon count distribution (equation A.21) for one emitting molecule can be written as:

$$
p^{(1)}(k)=\int_{0}^{\infty} \frac{\left(\eta_{I} I_{0} \beta \overline{P S F}(\vec{r})\right)^{k} e^{-\eta_{I} I_{0} \beta \overline{P S F}(\vec{r})}}{k !} p(\vec{r}) d \vec{r},
$$

which is equivalent to write:

$$
p^{(1)}(k)=\int_{0}^{\infty} \operatorname{Poi}\left(k, \eta_{I} I_{0} \beta \overline{P S F}(\vec{r})\right) p(\vec{r}) d \vec{r}
$$

In a first approach, a single emitting particle is assumed to diffuse inside a reference volume $V_{0}$. Because it is equiprobable to find the particle in any position inside $V_{0}$, the probability $p(\vec{r})$ is given by $1 / V_{0}$ if the particle is in $V_{0}$, or 0 otherwise. We can also define the molecular brightness $b$ of a molecule in the center of the PSF as:

$$
b=I_{0} \beta \eta_{I}=\eta_{I} I_{D}(\vec{r}) / \overline{P S F}(\vec{r}),
$$

Thus, equation A.27 becomes:

$$
p^{(1)}\left(k, V_{0}, b\right)=\int_{V_{0}} \operatorname{Poi}(k, b \overline{P S F}(\vec{r})) p(\vec{r}) d \vec{r}=\frac{1}{V_{0}} \int_{V_{0}} \operatorname{Poi}(k, b \overline{P S F}(\vec{r})) d \vec{r},
$$

The photon count distribution for one particle can be considered as the weighed average of different Poissonian distributions, each with an average value equal to $b \overrightarrow{P S F}(\vec{r})$, over $V_{0}$. When a fixed particle at a position $\overrightarrow{r_{0}}$ is considered, the $\mathrm{PCH}$ is a single Poissonian distribution:

$$
p^{\text {fixed }}\left(k, \overrightarrow{r_{0}}\right)=\operatorname{Poi}\left(k, b \overline{P S F}\left(\overrightarrow{r_{0}}\right)\right),
$$

where the average value of the distribution is $b \overline{P S F}\left(\overrightarrow{r_{0}}\right)$. The average photon count, $\langle k\rangle$, of the $p^{(1)}\left(k, V_{0}, b\right)$ is:

$$
\langle k\rangle=\frac{b}{V_{0}} \int_{V_{0}} \overline{P S F}(\vec{r}) d \vec{r}=b \frac{V_{\mathrm{PSF}}}{V_{0}} .
$$

The average photon count is determined by the brightness and the probability to find the molecule within the volume of the PSF. The molecular brightness $b$ is dependent on the binning time $T$, but the ratio $b / T$ is independent of the experimental binning time. The unit for this ratio is counts per seconds per molecule (cpsm) or simpler just count per second (cps).

To explicitly calculate the probability distribution for a single molecule, $p^{(1)}\left(k, V_{0}, b\right)$, the integration needs to be solved. For mathematical convenience, equation A.29 is integrated over all space. Since the reference volume $V_{0}$ is chosen so that it contains the PSF, there are no photons excited outside $V_{0}$, thus extending the 
integration limits to infinity does not change $p^{(1)}$, unless $k=0$. The probability of receiving 0 photon counts is then calculated by normalizing the probability distribution: $p^{(1)}\left(0, V_{0}, b\right)=1-\sum_{k=1}^{\infty} p^{(1)}\left(k, V_{0}, b\right)$. The following equation are obtained integrating over all space and they are valid only for $k>0$. Knowing the analytical form of the confocal PSF (equation A.23), the analytical integration over all space of the photon count distribution (equation A.29) for one emitting particle diffusing on $V_{0}$ leads to:

$$
p^{(1)}\left(k, V_{0}, b\right)=\frac{1}{V_{0}} \frac{\pi \omega_{0}^{2} z_{0}}{k !} \int_{0}^{\infty} \gamma\left(k, b e^{-2 x^{2}}\right) d x, \text { for } k>0
$$

where $\gamma$ represents the incomplete $\gamma$-function.

Until now, only the case of a single diffusing molecule was considered. If two independent molecules of the same species are diffusing within $V_{0}$, we can simply write the $\mathrm{PCH}$ with $\overrightarrow{r_{1}}$ and $\overrightarrow{r_{2}}$ to account for the two particles:

$$
p^{(2)}\left(k, V_{0}, b\right)=\iint \operatorname{Poi}\left(k, b \overline{P S F}\left(\overrightarrow{r_{1}}\right)+b \overline{P S F}\left(\overrightarrow{r_{2}}\right)\right) p\left(\overrightarrow{r_{1}}\right) p\left(\overrightarrow{r_{2}}\right) d \overrightarrow{r_{1}} d \overrightarrow{r_{2}},
$$

Practically to calculate the $\mathrm{PCH}$ for two diffusing independent identical particles $\left(p^{(2)}\left(k, V_{0}, b\right)\right)$ the Poissonian distribution calculated with the combined intensity of both particles, is averaged over all the possible positions in all space. The two particle case can be generalized to describe the photon count distribution for $N$ particles. If there are $N$ independent particles in $V_{0}$ with identical brightness, at position $\vec{r}_{n}$, then the PCH becomes:

$$
p^{(N)}\left(k, V_{0}, b\right)=\int \cdots \int \operatorname{Poi}\left(k, b \sum_{i=1}^{N} \overline{P S F}\left(\vec{r}_{i}\right)\right) p(\vec{r})_{1} \ldots p\left(\vec{r}_{N}\right) d \vec{r}_{1} \ldots d\left(\vec{r}_{N}\right),
$$

If it is assumed that the particles are non-interacting, the $N$ variables can be treated as statistically independent. The probability distribution of the sum of statistical independent variables can be then expressed as the convolution of $N$ individual probability distribution functions. The PCH for two particles can then be written as:

$$
p^{(2)}\left(k, V_{0}, b\right)=\left(p^{(1)} \otimes p^{(1)}\right)\left(k, V_{0}, b\right)=\sum_{r=0}^{\infty} p^{(1)}\left(k-r, V_{0}, b\right) p^{(1)}\left(k, V_{0}, b\right) .
$$

The PCH for $N$ identical and independent particles is also calculated applying the convolution $N$-times on the distribution for a single particle:

$$
p^{(N)}\left(k, V_{0}, b\right)=\left(p^{(1)} \otimes \cdots \otimes p^{(1)}\right)\left(k, V_{0}, b\right),
$$

Convoluting the single $\mathrm{PCH} N$-times is equivalent of evaluating the $3 \mathrm{~N}$-dimensional integral of equation A.34, but computationally is much more advantageous. 
So far, we have calculated the $\mathrm{PCH}$ only for particles diffusing in a confined volume $V_{0}$, however, in experiments the particles will move in an open space. Thus, we actually have an open system where particles are entering and leaving the sub-volume $V_{0}$. If the reference volume $V_{0}$ is chosen much smaller then the open system, the number of fluctuating particles in $V_{0}$ is governed by Poisson statistics:

$$
p_{\#}(N)=\operatorname{Poi}(N, \bar{N}),
$$

where $N$ is the actual number of molecules in the reference volume and $\bar{N}$ is the average number of molecules in $V_{0}$. The average number of molecules, $\bar{N}$, is related to the concentration of the sample as:

$$
\bar{N}=C V_{0} N_{A},
$$

where $N_{A}$ is the Avogadro's number. The final step in calculating the $\mathrm{PCH}$ for an open system is averaging the individual probability function for $N$ particles $\left(p^{(N)}\left(k, V_{0}, b\right)\right)$ weighted by the Poissonian probability of observing $\mathrm{N}$ particles $\left(p_{\#}(N)\right)$ :

$$
\begin{gathered}
\Pi\left(k, \bar{N}_{P S F}, b\right) \equiv \hat{p}\left(k, V_{0}, \bar{N}, b\right)=\left\langle p^{(N)}\left(k, V_{0}, b\right)\right\rangle_{N} \\
=\sum_{N=0}^{\infty} p^{(N)}\left(k, V_{0}, b\right) p_{\#}(N),
\end{gathered}
$$

The function $\hat{p}\left(k, V_{0}, \bar{N}, b\right)$ describes the probability of observing $k$ photon counts in an open system for a solution with a particle concentration of $c=$ $\bar{N} /\left(V_{0} N_{A}\right)$. Actually, the photon count probability for an open system is independent on the reference volume $V_{0}$. For this reason, the $\mathrm{PCH}$ for an open system should be referenced to the sample concentration, which is independent of $V_{0}$, or to a standard volume with a physical meaning. Usually, the convention used in FCS is followed also for PCH. The volume of the PSF in the FCS theory (Section A.2) serves as the standard volume which connects the average number of molecules $N$ within the PSF and the amplitude of the autocorrelation function $G(0)$. Therefore, the PCH for an open system is defined in equation A.39 as $\Pi\left(k, \bar{N}_{P S F}, b\right)$, where $\bar{N}_{P S F}$ is the average number of molecule in the PSF volume. Changing $\bar{N}$ with $\bar{N}_{P S F}$, in equation A.39, is justified because $c=\bar{N}_{P S F} /\left(V_{P S F} N_{A}\right)=\bar{N} / V_{0} N_{A}$. The average number of photon counts for an open system can be calculated from $\Pi\left(k, \bar{N}_{P S F}, b\right)$ as:

$$
\langle k\rangle=b \bar{N}_{P S F} .
$$

We can demonstrate that the $\mathrm{PCH}$ for an open system is independent of the reference volume. The probability $p^{(1)}\left(k, V_{0}, b\right)$ for a single molecule moving within $V_{0}$ depends on the reference volume. If the reference volume is changed from $V_{0}$ to $V_{1}$, where $V_{0}=f V_{1}$, the photon count distribution has also to be transformed. The transformation of the probability is defined as:

$$
p^{(1)}\left(k, V_{1}, b\right)=f p^{(1)}\left(k, V_{0}, b\right)+(1-f) \delta(k),
$$


where $\delta(k)$ is 1 for $k=1$, and 0 otherwise. When $V_{1}$ is bigger than $V_{0}, f$ can be considered as the probability of finding the molecule inside the smaller volume $V_{0}$. Thus, equation A.41 represents the joint probability of finding the molecule inside the volume $V_{0}$ with $p^{(1)}\left(k, V_{0}, b\right)$ and the joint probability of finding the molecule outside $V_{0}$, with the term related to $(1-f)$.

In contrast to the $\mathrm{PCH}$ for a single molecule in a confined volume $\left(p^{(1)}\left(k, V_{0}, b\right)\right)$, the probability distribution for an open system, $\left(\hat{p}\left(k, V_{0}, \bar{N}, b\right)\right)$, which describes the probability of $k$ photon counts from a freely diffusing chemical species with an average of molecules $\bar{N}$ in the volume $V_{0}$, is independent on the reference volume. We can explain intuitively this independence. In this case, when the reference volume is changed, the average number of molecules scales with the dimension of the reference volume. For example, changing the volume from $V_{0}$ to $V_{1}$, scales the average number of molecules from $\bar{N}_{0}$ to $\bar{N}_{1}=\bar{N}_{0} / f$. The choice of the reference volume for the open system case is of no importance as long as the average number of molecules in it also changes accordingly. For a practical purpose the reference volume is identical to the PSF volume for a confocal detection.

The three key assumptions so far for $\mathrm{PCH}$ are:

- The molecules do not move significantly during one time bin $T$, i.e. the fluorescence intensity is properly tracked over time $\left(T<\tau_{\text {fluc }}\right)$

- The molecular brightness is constant in time (no reactions and no photophysics)

- if $N$ particles are diffusing within the reference volume, they are independent and identical

If two or more different chemical species are present in the system, the differences in the molecular properties, such as the quantum yield or the excitation probability, have to be taken into consideration for the PCH. All the differences between the different species can be absorbed into the brightness, which will be different for each specie.

If, for example, the sample is composed by two species $N_{1}$ and $N_{2}$ with two different brightnesses $b_{1}$ and $b_{2}$ the $\mathrm{PCH}$ is given by:

$$
\begin{array}{r}
p^{\left(N_{1}, N_{2}\right)}\left(k, V_{0}, b_{1}, b_{2}\right)=\int \cdots \int p\left(\vec{r}_{i}\right) d\left(\vec{r}_{i}\right) \ldots p\left(\vec{r}_{j}\right) d\left(\vec{r}_{j}\right) \\
\operatorname{Poi}\left(k, b_{1} \sum_{i=1}^{N_{1}} \overline{P S F}\left(\vec{r}_{i}\right)+b_{2} \sum_{j=1}^{N_{2}} \overline{P S F}\left(\vec{r}_{j}\right)\right),
\end{array}
$$

But if the two species are assumed to be independent, we can write the final $\mathrm{PCH}$ as the convolution between the $\mathrm{PCH}$ functions of the individual single species, as:

$$
\Pi\left(k, \bar{N}_{1}, \bar{N}_{2}, b_{1}, b_{2}\right)=\Pi\left(k, \bar{N}_{1}, b_{1}\right) \otimes \Pi\left(k, \bar{N}_{2}, b_{2}\right) .
$$


which is again more convenient computationally than solving the integral in equation A.42. For more than two independent components, the PCH is the convolution of all the individual photon count histograms. 


\section{Bibliography}

[1] M. Goedert "Alpha-synuclein and neurodegenerative diseases." In: Nature Reviews Neuroscience, 2001, 2(7), pp: 492-501

[2] A. Horwich "Protein aggregation in disease: a role for folding intermediates forming specific multimeric interactions" In: The Journal of Clinical Investigation, 2002, 110(9), pp: 1221-1232

[3] K. L. J. Moreau "Protein misfolding and aggregation in cataract disease and prospects for prevention." In: Trends in molecular medicine, 2012, 18(5), pp: $273-282$.

[4] C. B. Lücking and A. Bric "Alpha-synuclein and Parkinson's diseases." In: Cellular and Molecular Life Sciences, 2000, 57(13), pp:1894-1908

[5] V. N. Uversky "Neuropathology, biochemistry, and biophysics of alphasynuclein aggregation." In: Journal of Neurochemistry, 2007, 103, pp: 1737, doi:10.1111/j.1471-4159.2007.04764.x

[6] J. R. Lakowicz. "Principles of fluorescence spectroscopy". Kluwer Academic Plenum Press, New York., 1999

[7] E.L. Elson,D. Magde. "Fluorescence correlation spectroscopy I. Conceptual basis and theory". In: Biopolymers, 1974, 13, pp: 1-27.

[8] Y. Chen, J. D. Müller, P. T. C. So and E. Gratton. "The Photon Counting Histogram in Fluorescence Fluctuation Spectroscopy". In: Biophysical Journal, 1999, 77, pp: 553-567.

[9] P. Kask, K. Palo, D. Ullmann and K. Gall "Fluorescence-intensity distribution analysis and its application in biomolecular detection technology." In: Proceedings of the National Academy of Sciences, 1999, 96, pp: 13756-13761

[10] M. Pitschke, R. Prior, M. Haupt and D. Riesner "Detection of single amyloid beta-protein aggregates in the cerebrospinal fluid of Alzheimer's patients by 
fluorescence correlation spectroscopy" In: Nature Medicine, 1998, 4(7), pp: $832-834$

[11] Y. Chen, L. Wei and J.D. Müller "Probing protein oligomerization in living cells with fluorescence fluctuation spectroscopy." In: Proceedings of the National Academy of Sciences, 2003, 100(26), pp: 15492-15497

[12] C. R. Nayak and A. D. Rutenberg "Quantification of Fluorophore Copy Number from Intrinsic Fluctuations during Fluorescence Photobleaching" In: Biophysical Journal, 2011, 101(9), pp: 2284-2293

[13] J. Chen, D. Chen, Y. Xie, T. Yuan and X. Chen "Progress of Microfluidics for Biology and Medicine." In: Nano-Micro Letters, 2013, 5(1), pp: 66-80

[14] M. E. Brennich, J. F. Nolting, C. Dammann, B. Nöding, S. Bauch, H. Herrmann, T. Pfohl and S. Köster "Dynamics of Intermediate Filament Assembly Followed in Micro-Flow by Small Angle X-Ray Scattering." In: Lab on a Chip, 2011, 11, pp: 708-716

[15] O. Saldanha, M. E. Brennich, M. Burghammer, H. Herrmann and S. Köster "The filament forming reactions of vimentin tetramers studied in a serialinlet microflow device by small angle x-ray scattering." In: Biomicrofluidics, $2016,10(2)$

[16] Y. Schaerli, R. C. Wootton, T. Robinson, V. Stein, C. Dunsby,M. A. A. Neil, P. M. W. French, A. J. deMello, C. Abell and F. Hollfelder. "ContinuousFlow Polymerase Chain Reaction of Single-Copy DNA in Microfluidic Microdroplets" In: Analytical Chemistry, 2009, 81(1), pp: 302-306

[17] D. M. Jameson, J. A. Ross and J. P. Albanesi. "Fluorescence fluctuation spectroscopy: ushering in a new age of enlightenment for cellular dynamics" In: Biophysical Review, 2009, 1, pp:105--118, DOI 10.1007/s12551-0090013-8

[18] R. Brown. " A brief account of microscopical observations made in the months of June, July and August 1827 on the particles contained in the pollen of plants; and on the general existence of active molecules in organic and inorganic bodies." In: The Philosophical Magazine, 1868, London

[19] E.L. Elson. "Quick tour of fluorescence correlation spectroscopy from its inception". In: Journal of Biomedical Optics, 2004, 9, pp: 857-864.

[20] B. D. Slaughter and R. Li "Toward Quantitative "In Vivo Biochemistry" with Fluorescence Fluctuation Spectroscopy" In: Molecular Biology of the Cell, 2010, 21(24), pp: 4306-4311 
[21] D.E. Madge, E.L. Elson and W.W. Webb "Thermodynamics fluctuations in a reacting system: measurement by fluorescence correlation spectroscopy." In: Physical Review Letters, 1972, 29, pp: 705-709

[22] D.E. Madge, E.L. Elson and W.W. Webb. "Fluorescence correlation spectroscopy. II. An experimental realization." In: Biopolymers, 1974, 13, pp: $29-61$

[23] H. Qian and E.L. Elson "Analysis of confocal laser-microscope optics for 3-D fluorescence correlation spectroscopy". In: Applied Optics, 1991, 30, pp:1185-1195.

[24] K.M. Berland and E. Gratton "Two-photon fluorescence correlation spectroscopy: method and application to the intracellular environment". In: Biophysics Journal, 1995, 68, pp:694-701.

[25] M. Ehrenberg and R. Rigler "Rotational Brownian motion and fluorescence intensity fluctuations". In: Chemical Physics, 1974, 4, pp: 390-401

[26] N.L. Thompson, D. Axelrod. "Immunoglobulin surface-binding kinetics studied by total internal reflection with fluorescence correlation spectroscopy". In: Biophysical Journal, 1983, 43, pp: 103-114

[27] D. Magde, W.W. Webb, E.L. Elson "Fluorescence correlation spectroscopy. III. Uniform translation and laminar flow" In: Biopolymers, 1978, 17, pp: $361-376$

[28] E. Rhoades, T.F. Ramlall, W.W. Webb and D. Eliezer "Quantification of alpha-synuclein binding to lipid vesicles using fluorescence correlation spectroscopy." In: Biophysical Journal, 2006, 90, pp:4692-4700

[29] N.O. Petersen, P.L. Hoddelius, P.W. Wiseman, O. Seger, K.E. Magnusson "Quantification of membrane receptor distributions by image correlation spectroscopy: concept and application". In: Biophysical Journal, 1993, 65, pp:1135-1146

[30] L. N. Thompson, X. Wang and P. Navaratnarajah "Total Internal Reflection with Fluorescence correlation Spectroscopy: applications to substratesupported planar membranes". In: Journal of Structure Biology, 2009, 168(1), pp: $95-106$

[31] L. Kastrup, H. Blom, C. Eggeling, and S. W. Hell "Fluorescence Fluctuation Spectroscopy in Subdiffraction Focal Volumes". In: Physical Review Letters, 2005, 94.

[32] A.G. Palmer and N. L. Thompson "Molecular aggregation characterized by high order autocorrelation in fluorescence correlation spectroscopy." In: Biophysical Journal, 1987, 52, pp: 257-270 
[33] J.D. Müller "Cumulant analysis in fluorescence fluctuation spectroscopy." In: Biophysical Journal, 2004, 86, pp: 3981-3992

[34] M. A. Digman, R. Dalal, A. F. Horwitz and E. Gratton. "Mapping the number of molecules and brightness in the laser scanning microscope." In: Biophysical Journal, 2008, 94, pp: 2320-2332

[35] A.G. Palmer and N.L. Thompson "Molecular aggregation characterized by high order autocorrelation in fluorescence correlation spectroscopy" In: Biophysical Journal, 1987, 52(2), pp: 257-270

[36] E. Banachowicz, A. Patkowski, G. Meier, K. Klameckaand J. Gapiński "Successful FCS experiment in nonstandard conditions." In: Langmuir, 2014, 29, 30(29), pp: 8945-55. doi: 10.1021/la5015708.

[37] J. W. D. Comeau, D. L. Kolin and P. W. Wiseman "Accurate measurements of protein interactions in cells via improved spatial image cross-correlation spectroscopy." In: Molecular BioSystems, 2008, 4(6), pp: 672

[38] M. A. Digman and E. Gratton "Analysis of diffusion and binding in cells using the RICS approach." In: Microscopy Research and Technique, 2009, 72(4), pp: 323-332

[39] Y. Chen, B. Wu, K. Musier-Forsyth, L. M. Mansky and J D. Müller "Fluorescence Fluctuation Spectroscopy on Viral-Like Particles Reveals Variable Gag Stoichiometry" In: Biophysical Journal, 2009, 96(5), pp: 1961-1969

[40] C. Dong and J. Ren "Coupling of fluorescence correlation spectroscopy with capillary and microchannel analytical systems and its applications" In: Electrophoresis, 2014, 35(16), pp: 2267-2278

[41] A. Jablonski "Über den Mechanisms des Photolumineszenz von Farbstoffphosphoren" In: Zeitschrift für Physik, 1935, 94, pp: 38-46

[42] G. G. Stokes "On the change of refrangibility of light." In: Philosophical Transactions of the Royal Society (London), 1852, 142, pp: 463-562

[43] J.W. Lichtman and J-A. Conchello "Fluorescence Microscopy". In: Nature Methods, 2005, 2(12):910-9.

[44] M. Erenberg and R. Rigler. "Rotational Brownian motion and fluorescence intensity fluctuations". In: Chemical Physics, 1974, 4, pp:390-401.

[45] J. Widengren and R. Rigle. "Photodynamic properties of green fluorescent proteins investigated by fluorescence correlation spectroscopy". In: Chemical Physics, 1999, 250, pp: 171-186. 
[46] L Mandel "Fluctuations of Photon Beams: The Distribution of the PhotoElectrons". Proceedings of the Physical Society, 1959, 74(233)

[47] M. Zhao, L. Jin, B. Chen, Y. Ding, H. Ma and D. Chen. "Afterpulsing and Its Correction in Fluorescence Correlation Spectroscopy Experiments". In: Applied Optics, 2003, 42.19, pp: 4031-4036.

[48] M. Gösch, H. Blom, J. Holm, T. Heino and R. Rigler "Hydrodynamic Flow Profiling in Microchannel Structures by Single Molecule Fluorescence Correlation Spectroscopy." In: Analytical Chemistry, 2000, 72(14), pp:3260-3265, doi: https://doi.org/10.1021/ac991448p

[49] C. M. Brown, R. B. Dalal, B. Herbert, M. A. Digman, A. R. Horwitz and E. Gratton. "Raster image correlation spectroscopy (RICS) for measuring fast protein dynamics and concentrations with a commercial laser scanning confocal microscope". In: Journal of Microscopy, 2007, 22, pp:78-92.

[50] W. Feller "An introduction to probability theory and its applications", John Wiley and Sons, 1957

[51] B. Huang,T. D: Perroud and R. N. Zare. "Photon Counting Histogram: One-Photon Excitation". In: ChemPhysChem, 5, (2004), pp: 1523-1531.

[52] L. N. Hillesheim and J. D. Müller "The photon Counting Histogram in Fluorescence Fluctuation Spectroscopy with Non-Ideal Photodetectors". In: Biophysical Journal, 2003, 85, pp: 1948-1958.

[53] J. Johnson, Y. Chen and J. D. Müller "Characterization of Brightness and Stoichiometry of Bright Particles by Flow-Fluorescence Fluctuation Spectroscopy". In: Biophysical Journal, 2010, 99, pp: 3084-3092.

[54] T.S. Ulmer, A. Bax, N.B. Cole and R.L. Nussbaum "Structure and Dynamics of Micelle-bound Human alpha-Synuclein" In: Journal of Biological Chemistry, 2004, 280(10), pp: 9595-9603. DOI:10.1074/jbc.m411805200

[55] M. D. Tuttle, G. Comellas, A. J. Nieuwkoop, D. J. Covell, D. A. Berthold, K. D. Kloepper, J. M. Courtney, J. K. Kim, A. M. Barclay, A. Kendall, W. Wan, G. Stubbs, C. D. Schwieters, V. M. Y. Lee, J. M. George and C. M. Rienstra "Solid-state NMR structure of a pathogenic fibril of full-length human alpha-synuclein" In: Nature Structural \&6 Molecular Biology, 2016, 23(5), pp: 409-415, DOI:10.1038/nsmb.3194

[56] J. D. Müller, Y. Chen and E. Gratton "Resolving Heterogeneity on the Single Molecular Level with the Photon-Counting Histogram." In: Biophysical Journal, 2000, 78(1), pp: 474-486 
[57] Y. Chen, J. Johnson, P. Macdonald, B. Wu and J. D. Müller "Chapter 16 Observing Protein Interactions and Their Stoichiometry in Living Cells by Brightness Analysis of Fluorescence Fluctuation Experiments." In: Single Molecule Tools: Fluorescence Based Approaches, Part A, 2010, 472, pp: 345-363

[58] J. B. Knight, A. Vishwanath, J. P. Brody, and R. H. Austin. "Hydrodynamic Focusing on a Silicon Chip: Mixing Nanoliters in Microseconds" In: Physical Review Letters, 1998, (80), pp: 3863-3866.

[59] G. Chirico, F. Olivini and S. Beretta "Fluorescence Excitation Volume in Two-Photon Microscopy by Autocorrelation Spectroscopy and Photon Counting Histogram." In: Applied Spectroscopy, 2000, 54(7), pp: 10841090. https://doi.org/10.1366/0003702001950607

[60] G. Malengo, A. Annapaola, S. Nicolai, E. Gratton, M. Zamai and V. R. Caiolfa "Fluorescence correlation spectroscopy and photon counting histogram on membrane proteins: functional dynamics of the glycosylphosphatidylinositol-anchored urokinase plasminogen activator receptor." In: Journal of Biomedical Optics, 2008, 13(3)

[61] X. Michalet, S. Weiss and M. Jäger "Single-Molecule Fluorescence Studies of Protein Folding and Conformational Dynamics" In: Chemical Reviews, 2006, 105, pp: 1785-1813

[62] T. D. Perroud, M. P. Bokoch and R. N. Zare "Cytochrome c conformations resolved by the photon counting histogram: Watching the alkaline transition with single-molecule sensitivity" In: Proceedings of the National Academy of Sciences, 2005, 102 (49), pp: 17570-17575; DOI: 10.1073/pnas.0508975102

[63] N. Terada, T. Shimozawa, S. Ishiwata and T. Funatsu "Size Distribution of Linear and Helical Polymers in Actin Solution Analyzed by Photon Counting Histogram". In: Biophysical Journal, 2007, 92(6), pp: 2162-2171, doi $=$ "https://doi.org/10.1529/biophysj.106.098871"

[64] S. Rammensee, U. Slotta, T. Scheibel and A. R. Bausch "Assembly mechanism of recombinant spider silk proteins" In: Proceedings of the National Academy of Sciences, 2008, 105(18), pp: 6590-6595

[65] R. Russell, I. S. Millett, M. W. Tate, L. W. Kwok, B. Nakatani, S. M. Gruner, S. G. J. Mochrie, V. Pande, S. Doniach, D. Herschlag and L. Pollack "Rapid compaction during RNA folding" In: Proceedings of the National Academy of Sciences, 2002, 99(7), pp: 4266-4271

[66] S. Yao and O. Bakajin "Improvements in Mixing Time and Mixing Uniformity in Devices Designed for Studies of Protein Folding Kinetics" In: Analytical Chemistry, 2007, 79(15), pp: 5753-5759 
[67] S. Seibt, S. With, A. Bernet, H.W. Schmidt and S. Förster "Hydrogelation Kinetics Measured in a Microfluidic Device with in Situ X-ray and Fluorescence Detection" In: Langmuir, 2018, 34(18), pp: 5535-5544

[68] D. C. Duffy, J. C. McDonald, O. J. A. Schueller, and G. M. Whitesides. "Rapid Prototyping of Microfluidic Systems in Poly(dimethylsiloxane)". In: Analytical Chemistry, 70.23 (Dec. 1998), pp: 4974-4984. doi: $10.1021 /$ ac980656z

[69] H. Song, D. L. Chen, and R. F. Ismagilov. "Reactions in droplets in microfluidics channels". In: Angewandte Chemie (International ed. in English). 45(44):7336-7356, 2006.

[70] Pijush Kundu Ira Cohen David Dowling. "Fluid Mechanics." Elsevier, 2012

[71] Henrik Bruus "Theoretical Microfluidics" Oxford University Press, 2008.

[72] D. J. Beebe, G. A. Mensing, and G. M. Walker. "Physics and applications of microfluidics in biology." In: Annual Review of Biomedical Engineering, 2002, (4), pp:261-286, doi: 10.1146/annurev.bioeng.4.112601.125916.

[73] T. M. Squires and S. R. Quake "Microfluidics: Fluid Physics at the Nanoliter Scale" In: Reviews of Modern Physics, 2005, (77), pp: 977-1026, doi:10.1103/RevModPhys.77.977

[74] A. S. Kane, A. Hoffmann, P. Baumgärtel, R. Seckler, G. Reichardt, D. A. Horsley, B. Schuler, and O. Bakajin. "Microfluidic Mixers for the Investigation of Rapid Protein Folding Kinetics Using Synchrotron Radiation Circular Dichroism Spectroscopy". In: Analytical Chemistry, 2008,(80), pp: 9534-9541 doi: 10.1021/ac801764r.

[75] H. Y. Park, X. Qiu, E. Rhoades, J. Korlach, L. W. Kwok, W. R. Zipfel, W. W. Webb and L. Pollack. "Achieving Uniform Mixing in a Microfluidic Device: Hydrodynamic Focusing Prior to Mixing" In: Analytical Chemistry, 2006, (78), pp: 4465-4473. doi: 10.1021/ac060572n

[76] M. S. Williams, K. J. Longmuir, and P. Yager."A Practical Guide to the Staggered Herringbone Mixer" In: Lab on a Chip, 2008, pp: 1121-1129

[77] W. Saadi, S. W. Rhee, F. Lin, B. Vahidi, B. G. Chung and N. L. Jeon "Generation of stable concentration gradients in 2D and 3D environments using a microfluidic ladder chamber" In: Biomedical Microdevices, 2007, 9(5), pp: 627-635

[78] D. H. Boal, "Mechanics of the Cell", second edition, Cambridge University Press, Cambridge, 2012. 
[79] B. Alberts, "Molecular Biology of the Cell", sixth edition. Garland Science Taylor and Francis Group, New York, 2015

[80] J. F. Conway and D. A. Parry, "Intermediate Filament Structure. 3. Analysis of Sequence Homologies." In: International Journal of Biological Macromolecules, 1988, 10, pp: 79-98.

[81] H. Herrmann and U. Aebi, "Intermediate Filaments and Their Associates. Multi-Talented Structural Elements Specifying Cytoarchitecture and Cytodynamics." In: Current Opinion in Cell Biology, 2000, (12), pp: 79-90.

[82] H. Herrmann, S. V. Strelkov, P. Burkhard and U. Aebi, "Intermediate Filaments: Primary Determinants of Cell Architecture and Plasticity." In: Journal of Clinical Investigation, 2009, (119), pp:1772-1783

[83] J. Block,V. Schroeder, P. Pawelzyk, N. Willenbacher and S. Köster, "Physical Properties of Cytoplasmic Intermediate Filaments." In: Biochimica et Biophysica Acta, 2015, (1853), pp: 3053-3064

[84] M. Hatzfeld and W.W. Franke, " Pair Formation and Promiscuity of Cytokeratins. Formation in Vitro of Heterotypic Complexes and IntermediateSized Filaments by Homologous and Heterologous Recombinations of Purified Polypeptides." In: Journal of Cellular Biology, 1985, (101), pp: 18261841.

[85] A. C. Steven,J. F. Hainfeld, B. L. Trus, J. S. Wall and P. M. Steinert, " The Distribution of Mass in Heteropolymer Intermediate Filaments Assembled in Vitro Stem Analysis of Vimentin/Desmin and Bovine Epidermal Keratin." In: Journal of Biological Chemistry, 1983, (258), pp: 8323-8329.

[86] A. Engel, R. Eichner and U.Aebi, "Polymorphism of Reconstituted Human Epidermal Keratin Filaments. Determination of their Mass-Per-Length and Width by Scanning Transmission Electron Microscopy (STEM)." In: Journal of Molecular Structure, 1985, (90), pp: 323-335.

[87] C.G. Lopez, O. Saldanha, H. Klaus and S. Köster. "Light scattering of intermediate filament assembly." In: Proceedings of the National Academy of Sciences, 2016, 113 (40), pp:11152-11157, DOI: 10.1073/pnas.1606372113

[88] H. Herrmann, M. Häner, M. Brettel, N. Ku and U. Aebi, " Characterization of Distinct Early Assembly Units of Different Intermediate Filament Proteins." In: Journal of Molecular Biology, 1999, (286), pp: 1403-1420.

[89] R. Kirmse, S. Portet, N. Mücke, U. Aebi, H. Herrmann an J. Langowski "A Quantitative Kinetic Model for the in Vitro Assembly of Intermediate Filaments from Tetrameric Vimentin." In: Journal of Molecular Chemistry, 2007, (282), pp: 18563-18572 
[90] E. Czeizler, A. Mizera, E. Czeizler, R. Back, J. E. Eriksson and I. Petre "Quantitative Analysis of the Self-Assembly Strategies of Intermediate Filaments from Tetrameric Vimentin" In: Transactions on Computational Biology and Bioinformatics, 2012, 9(3), pp: 885-898

[91] S. Portet, N. Mücke, R. Kirmse, J. Langowski, M. Beil and H. "Vimentin Intermediate Filament Formation: In Vitro Measurement and Mathematical Modeling of the Filament Length Distribution during Assembly", Langmuir, 2009, (25), pp: 8817-8823.

[92] A. V. Sokolova, L. Kreplak, T. Wedig, N. M"ucke, D. I. Svergun, H. Herrmann, U. Aebi, and S. V. Strelkov "Monitoring Intermediate Filament Assembly by Small-Angle X-Ray Scattering Reveals the Molecular Architecture of Assembly Intermediates." In: Proceedings of the National Academy of Sciences, 2006, (103), pp: 16206-16211

[93] M. E. Brennich, S. Bauch, U. Vainio, T. Wedig, H. Herrmann and S. Köster "Impact of Ion Valency on the Assembly of Vimentin Studied by Quantitative Small Angle X-Ray Scattering." In: Soft Matter, 2014, (10), pp: 2059-2068

[94] C. G. Lopez, O. Saldanha, A. Aufderhorst-Roberts, C. Martinez-Torres, M. Kuijs, G. H. Koenderink, S. Köster and K. Huber "Effect of ionic strength on the structure and elongational kinetics of vimentin filaments." In: Soft Matter, 2018, 14(42), pp: 8445-8454

[95] N. Mücke, T. Wedig, A. Bürer, L. N. Marekov, P. M. Steinert, J. Langowski, U. Aebi and H. Herrmann "Molecular and Biophysical Characterization of Assembly-Starter Units of Human Vimentin" In: Journal of Molecular Biology, 2004, 340(1), pp: 97-114

[96] N. Mücke, L. Kämmerer, S. Winheim, R. Kirmse, J. Krieger, M. Mildenberger, J. Bassles, E. Hurt, W. H. Goldmann, U. Aebi, K. Toth, J. Langowski and H. Herrmann, "Assembly Kinetics of Vimentin Tetramers to Unit-Length Filaments: A Stopped-Flow Study." In: Biophysical Journal, 2018, 114, (10), pp: 2408-2418.

[97] J. M. George "The synucleins." In: Genome Biology, 2001, 3(1), pp:3002.13002.6

[98] E.R. Kandel, J.H. Schwaetz and T.M. Jessel "Principles of Neural Science (4th edition)", 200, New York: McGraw-Hill. ISBN 978-0-8385-7701-1.

[99] B. G. Wilhelm, S. Mandad, S. Truckenbrodt, K. Kröhnert, C. Schäfer, B. Rammner, S. J. Koo, G. A. Classen, M. Krauss, V. Haucke, H. Urlaub, S. O. Rizzoli, "Composition of isolated synaptic boutons reveals the amounts of vesicle trafficking proteins." In: Science, 2014, 344, pp: 1023-1028 
[100] M.G. Spillantini, M.L. Schmidt, V.M. Lee, J.Q. Trojanowski, R. Jakes and M. Goedert "Alpha-synuclein in Lewy bodies" In: Nature, 1997, 338 (6645), pp: $838-840$

[101] C.C. Jao, B.G. Hegde, J. Chen, I.S. Haworth and R. Langen "Structure of membrane-bound alpha-synuclein from site-directed spin labeling and computational refinement." In: Proceedings of the National Academy of Sciences, 2008, 105(50), pp:19666-71, doi:10.1073/pnas.0807826105.

[102] J. Burré, M. Sharma, T. Tsetsenis, V. Buchman, M. R. Etherton and T. C. Südhof "Alpha-Synuclein Promotes SNARE-Complex Assembly in Vivo and in Vitro." In: Science, 2010, pp: 1663-1667

[103] D. Scott and S. Roy "Alpha-Synuclein inhibits intersynaptic vesicle mobility and maintains recycling-pool homeostasis." In: Journal of Neuroscience, 2012, 32, pp: 10129-10135

[104] J. Sun, L. Wang, H. B., S. Premi, U. Das, E. R. Chapman and S. Roy "Functional cooperation of alpha-synuclein and VAMP2 in synaptic vesicle recycling." In: Proceedings of the National Academy of Sciences, 2019, 116 (23), pp: 11113-11115, DOI: 10.1073/pnas.1903049116

[105] S. Chandra, G. Gallardo, R. Fernandez-Chacon, O. M. Schluter and T. Sudhof "Alpha-synuclein cooperates with CSPalpha in preventing neurodegeneration." In: Cell, 2005, 123, pp: 383-396.

[106] K. K. Dev, K. Hofele, S. Barbieri, V. Buchman and H. Van Der Putten "Part II: alpha-synuclein and its molecular pathophysiological role in neurodegenerative disease." In: Neuropharmacology, 2003, 45, pp:14--44.

[107] S. Chandra, X. Chen, J. Rizo, R. Jahn and T. C. Sudhof. " A broken alphahelix in folded alpha-synuclein." In: Journal of Biological Chemistry, 2003, 278, pp: 15313-15318.

[108] S. Takamori, M. Holt,K. Stenius, E. A. Lemke, M. Grønborg, D. Riedel, H. Urlaub,S. Schenck, B. Bruügger, P. Ringler, S. A. Müller, B. Rammner, F. Gräter, J. S. Hub, B. L. De Groot, G. Mieskes, Y. Moriyama, J. Klingauf, H. Grubmüller, J. Heuser, F. Wieland and R. Jahn "Molecular Anatomy of a Trafficking Organelle" In: Cell, 2006, 127, pp: 831-846

[109] S. O. Rizzoli and W. J. Betz "Synaptic vesicle pools" In: Nature Reviews Neuroscience, 2005, 6, pp:57-69

[110] Thomas C. Sudhof "The synaptic vesicle cycle." In: Annual Review of Neuroscience, 2004, 27(1), pp: 509-547

[111] Jacqueline Burré "The Synaptic Function of Alpha-Synuclein." In: Journal of Parkinsons Disease, 2015, 5(4), pp: 699-713, doi: 10.3233/JPD-150642 
[112] website: https://healthtalk.unchealthcare.org/

[113] J. Block, H. Witt, A. Candelli, J.C. Danes, E.J.G. Peterman, G.J.L. Wuite, A. Janshoff and S. Köster. "Viscoelastic properties of vimentin originate from nonequilibrium conformational changes". In: Science Advances, 2018, 4.6, DOI: $10.1126 /$ sciadv.aat1161

[114] S. Winheim, A. R. Hieb, M. Silbermann, E. Surmann, T. Wedig, H. Herrmann, J. Langowski and and Norbert Mücke. "Deconstructing the Late Phase of Vimentin Assembly by Total Internal Reflection Fluorescence Microscopy (TIRFM)". In: PLOS ONE, 2011, 6.4, DOI:10.1371/journal.pone.0019202

[115] D. Duffy, J. Cooper McDonald, O. J. A. Schueller and G M. Whitesides. "Rapid Prototyping of Microfluidic Systems in Poly(dimethylsiloxane) ) titanium(IV) derivatives". In: Analytical Chemistry, 1998, 70(23), pp:49744984 .

[116] J. Cooper McDonald and George M. Whitesides. "Poly(dimethylsiloxane) as a Material for Fabricating Microfluidic Devices titanium(IV) derivatives". In: Accounts of Chemical Research, 2002, 35(7), pp: 491-499.

[117] E. Kim and Y. Xia and George M. Whitesides. "Micromolding in Capillaries: Applications in Material Science". In: Journal of the American Chemical Society, 1996, 35(24), pp: 5722-5731.

[118] Q. Chen, G. Li, Y. Nie et al. "Investigation and improvement of reversible microfluidic devices based on glass-PDMS-glass sandwich configuration". In: Microfluidics and Nanofluidics, 2014, 16(83), DOI: ttps://doi.org/10.1007/s10404-013-1222-9

[119] H. Herrmann, M. Häner, M. Brettel, S. A. Müller, K. N. Goldie, B. Fedtke, A. Lustig, W. W. Franke and U. Aebi. "Structure and Assembly Properties of the Intermediate Filament Protein Vimentin: The Role of its Head, Rod and Tail Domains" In: Journal of Molecular Biology, 1996, (264), pp: 933953

[120] S. V. Strelkov, H. Herrmann and U. Aebi "Molecular architecture of intermediate filaments." In: Bioessays, 2003, 25, pp: 243-251

[121] A. Chernyatina, S. Nicolet, U. Aebi, H. Herrmann, S. V. Strelkov "Atomic structure of the vimentin rod domain" In: Proceedings of the National Academy of Sciences, 2012, 109(34), pp: 13620-13625

[122] I. Hofmann, H. Herrmann and W. W. Franke. "Assembly and structure of calcium-induced thick vimentin filaments". In: European Journal of Cell Biology, 1991, (56), pp: 328-341 
[123] Pierre Clade، "PyDAQmx : A Python Interface to the National Instruments DAQmx Driver", "http://pythonhosted.org/PyDAQmx/"

[124] A. Edelstein, N. Amodaj, K. Hoover, R. Vale and N. Stuurman. "Computer Control of Microscopes Using Micro-Manager". In: Current Protocols in Molecular Biology, 2010, (56),14.20,doi = 10.1002/0471142727.mb1420s92

[125] V. Buschmann, B. Krämer and F. Koberling. "Quantitative FCS: Determination of the Confocal Volume by FCS and Bead Scanning with the MicroTime200". Application Note, PicoQuant GmbH Rainer Macdonald, Steffen Rüttinger, Physikalisch Technische Bundesanstalt, Germany

[126] C. B. Müller, A. Loman, V. Pacheco, F. Koberling, D. Willbold, W. Richtering, and J. Enderlein. "Precise Measurement of Diffusion by Multi-Color Dual-Focus Fluorescence Correlation Spectroscopy." In: EPL, 2008, 83.4, p. 46001, doi: 10.1209/0295-5075/83/46001

[127] Dertinger T., Ewers B. Unpublished results. PicoQuant GmbH (2008)

[128] J. Krieger and J. Langowski. " QuickFit 3.0 (Compiled: 2015-10-29, SVN: 4465): A Data Evaluation Application for Biophysics." 2010-2016

[129] C. Hodges, R.P. Kafle, J. D. Hoff and J.C. Meiners "Fluorescence Correlation Spectroscopy with Photobleaching Correction in Slowly Diffusing Systems". In: Journal of Fluorescence, 2018, 28, pp:505-511

[130] Viktor Schroeder "Fluorescence correlation spectroscopy for studying intermediate filament assembly" $\mathrm{PhD}$ dissertation, University of Göttingen, 2017, eDISS

[131] J. G. De La Torre, M. C. Lopez Martinez, and M. Mercedes Tirado. "Dimensions of Short, Rod like Macromolecules from Translational and Rotational Diffusion Coefficients. Study of the Gramicidin Dimer" In: Biopolymers, 1984, 23.4, pp: 611-615

[132] S. H. Pfeil, C. E. Wickersham, A. Hoffmann, and E. A. Lipman "A Microfluidic Mixing System for Single-Molecule Measurements" In: Review of Scientific Instruments, 2009, 80.5, pp: 055105-055105-9.

[133] W. Chen, L. J. Young, M. Lu, A. Zaccone, F. Ströhl, N. Yu, G. S. Kaminski Schierle, and C. F. Kaminski "Fluorescence Self-Quenching from Reporter Dyes Informs on the Structural Properties of Amyloid Clusters Formed in Vitro and in Cells." In: Nano Letters, 2017, 17(1), pp: 143--149, doi:10.1021/acs.nanolett.6b03686

[134] M. Denz, G. Brehm, C. Y. J. Hemonnot, H. Spears, A. Wittmeier, C. Cassini, O. Saldanha, E. Perego, A. Diaz, M. Burghammer and S. Köster 
"Cyclic olefin copolymer as an X-ray compatible material for microfluidic devices". In: Lab on a Chip, 2018, 18, pp: 171-178

[135] S. Köster, D. A. Weitz, R D. Goldman, U. Aebi, H.Herrmann "Intermediate filament mechanics in vitro and in the cell: from coiled coils to filaments, fibers and networks" In: Current Opinion in Cell Biology, 2015, 32, pp: $82-91$

[136] M. Kang, C. A. Day, A. K. Kenworthy and E. DiBenedetto. "Simplified equation to extract diffusion coefficients from confocal FRAP data." In: Traffic, 2012, 13, pp: 1589-1600

[137] Y. Chen, J D. Müller, Q. Ruan and E. Gratton. "Molecular Brightness Characterization of EGFP In Vivo by Fluorescence Fluctuation Spectroscopy" In: Biophysical Journal, 2002, 82(1), pp: 133-144

[138] P. Schwille, U. Haupts, S. Maiti and W. W. Webb "Molecular Dynamics in Living Cells Observed by Fluorescence Correlation Spectroscopy with Oneand Two-Photon Excitation" In: Biophysical Journal, 77(4), pp: 2251-2265

[139] M. Wachsmuth, W. Waldeck and J. Langowski "Anomalous diffusion of fluorescent probes inside living cell nuclei investigated by spatially-resolved fluorescence correlation spectroscopy11Edited by W. Baumeister" In: Journal of Molecular Biology, 2000, 298(4), pp: 677-689

[140] D. Milovanovic, Y. Wu, X. Bian, P.D. Camilli "A liquid phase of synapsin and lipid vesicles.", In: Science, 2018, 361, pp: 604-607

[141] T. Schikorski, and C. F. Stevens "Quantitative ultrastructural analysis of hippocampal excitatory synapses" In: Journal Neuroscience, 1997, 17, pp: $5858-5867$

[142] T. Schikorski, and C. F. Stevens "Morphological correlates of functionally defined synaptic vesicle populations." In: Nature Neuroscience, 2001, 4, pp: 391-395

[143] P. N. Hedde, M. Stakic and E. Gratton "Rapid Measurement of Molecular Transport and Interaction inside Living Cells Using Single Plane Illumination." In: Scientific Reports, 2014, 7048

[144] B. Ahmad, Y. Chen and L. J. Lapidus "Aggregation of alpha-synuclein is kinetically controlled by intramolecular diffusion" In: Proceedings of the National Academy of Sciences, 2012, 109(7), pp: 2336-2341, DOI:10.1073/pnas.1109526109

[145] R. Swaminathan, C.P. Hoang and A.S. Verkman "Photobleaching recovery and anisotropy decay of green fluorescent protein GFP-S65T in solution and 
cells: cytoplasmic viscosity probed by green fluorescent protein translational and rotational diffusion" In: Biophysical Journal, 1997, 72(4), pp: 19001907

[146] M. K. Knowles et all "Single secretory granules of live cells recruit syntaxin-1 and synaptosomal associated protein 25 (SNAP-25) in large copy numbers. " In: Proceedings of the National Academy of Sciences, 2010, 107, pp: 2081020815

[147] S. A. Kim, K. G. Heinze and P. Schwille "Fluorescence correlation spectroscopy in living cells" In: Nature Methods, 2007, 4(11), pp: 1548-7105

[148] R. G. Sadovsky, S. Brielle, D. Kaganovich and J. L. England "Measurement of Rapid Protein Diffusion in the Cytoplasm by Photo-Converted Intensity Profile Expansion" In: Cell Reports, 2017, 18(11), pp: 2795-2806

[149] C. Yeung, M. Shtrahman and X. Wu "Stick-and-Diffuse and Caged Diffusion: A Comparison of Two Models of Synaptic Vesicle Dynamics." In: Biophysical Journal, 2007, 92(7), pp: 2271-2280

[150] S. Nath, J. Meuvis, J. Hendrix, S. A. Carl and Y. Engelborghs "Early Aggregation Steps in alpha-Synuclein as Measured by FCS and FRET: Evidence for a Contagious Conformational Change." In: Biophysical Journal, 2010, 98(7), pp: 1302-1311

[151] V. N. Uversky, J. Li and A. L. Fink. "Evidence for a Partially Folded Intermediate in alpha-Synuclein Fibril Formation". In: The Journal of Biological Chemistry, 2001, 276, pp: 10737-10744

[152] J. Nidhi, B. Sujit, K. Sangeeta, D. Goutam, A. Mukhopadhyay and K. Chattopadhyay "Attenuation of the Early Events of alpha-Synuclein Aggregation: A Fluorescence Correlation Spectroscopy and Laser Scanning Microscopy Study in the Presence of Surface-Coated Fe3O4 Nanoparticles" In: American Chemical Society, 2015, 31(4), pp:7430-7463

[153] E. Middleton and E. Rhoades "Effects of Curvature and Composition on alpha-Synuclein Binding to Lipid Vesicles" In: Biophysical Journal, 2010, 99, pp: $2279-2288$

[154] R. Jahn and J. Boyken. "Molecular Regulation of Synaptic Release." In: Neuroscience in the 21st Century, Springer, New York, NY

[155] M. Cretich, G. Pirri, F. Damin, I. Solinas and M. Chiaria "A new polymeric coating for protein microarrays" In: Analytical Biochemistry, 2004, 332, pp:67-74 
[156] C. T Culbertson, S. C. Jacobson and J M. Ramsey "Diffusion coefficient measurements in microfluidic devices" In: Talanta, 2002, 56(2), pp: 365373

[157] V. Tokárová, A. Pittermannová and V. Král, P. Řezáčová and F. Štěpánek, "Feasibility and constraints of particle targeting using the antigen-antibody interaction". In: Nanoscale, 2013, 5(23), pp: 11490

[158] T. G. Papaioannou and C. Stefanadis "Vascular wall shear stress: basic principles and methods" In: Hellenic Journal Cardiology, 2005, 46, pp: 9 15

[159] Z. Qin, L. Kreplak and M. J. Buehler "Hierarchical Structure Controls Nanomechanical Properties of Vimentin Intermediate Filaments". In: PLOS ONE, 2009, 4, pp: 1-14

[160] H. Bär, S. V. Strelkov, G. Sjöberg, U. Aebi and H. Herrmann "The biology of desmin filaments: how do mutations affect their structure, assembly, and organisation?". In: Journal of Structural Biology, 2004, 148(2), pp: 137-152

[161] H. H. Goebel "Desmin-related neuromuscular disorders." In: Muscle $\&$ Nerve, 1995, 18(11), pp:1306-1320

[162] T. Deptuła, J. Buitenhuis, M. Jarzębski, P. Maciej and A. Patkowski and J. Gapinski "Size of Submicrometer Particles Measured by FCS: Correction of the Confocal Volume". In: Langmuir, 2015, 32(24), pp: 6681-6687

[163] J. B. Knight, A. Vishwanath, J. P. Brody and R. H. Austin "Hydrodynamic Focusing on a Silicon Chip: Mixing Nanoliters in Microseconds" In: Physical Review Letters, 1998, 80(17), pp: 3863-3866

[164] S. Köster, H. M. Evans, J. Y. Wong and T. Pfohl "An In Situ Study of Collagen Self-Assembly Processes" In: Biomacromolecules, 2008, 9(1), pp: 199-207

[165] D. S. Banks and C. Fradin "Anomalous Diffusion of Proteins Due to Molecular Crowding." In: Biophysical Journal, 2005, 89(5), pp: 2960-2971

[166] J. He, S.M. Guo, M. Bathe "Bayesian Approach to the Analysis of Fluorescence Correlation Spectroscopy Data I: Theory." In: Analytical Chemistry, 2012, 84(9), pp: 3871-3879

[167] T. Lichtenstern, N. Mücke, U. Aebi, M. Mauermann and H. Herrmann "Complex formation and kinetics of filament assembly exhibited by the simple epithelial keratins K8 and K18." In: Journal of Structural Biology, 2012, 177(1), pp: 54-62 
[168] R. Sandmann, S. Schwarz, G. Henriques, F. Rehfeldt and Sarah Köster "Micro-topography influences blood platelet spreading". In: Soft Matter, 2014, 10(14), pp: 2365-2371 


\section{List of Publications}

- Sofiia Reshetniak, Jan-Eike Ußling, Eleonora Perego, Burkhard Rammner, Thomas Schikorski, Eugenio F. Fornasiero, Sven Truckenbrodt, Sarah Köster and Silvio O. Rizzoli. A comparative analysis of the mobility of 45 proteins in the synaptic bouton. In press EMBO Journal, (2020).

- Jana Hanke, Christiane Ranke, Eleonora Perego and Sarah Köster. FHuman Blood Platelets Contract in Perpendicular Direction to Shear Flow. Soft Matter, 15, 2009-2019 (2019).

- Manuela Denz, Gerrit Brehm, Clément Y. J. Hémonnot, Heidi Spears, Andrew Wittmeier, Chiara Cassini, Oliva Saldanha, Eleonora Perego, Ana Diaz, Manfred Burghammer and Sarah Köster. Cyclic olefin copolymer as an X-ray compatible material for microfluidic devices. Lab on a Chip, 18, 171-178 (2018). 


\section{Short Curriculum Vitae}

Eleonora Perego, born $15^{\text {nd }}$ of January 1991 in Milano (Italy).

- 2015 - 2019 PhD in Physics, at the Institute for X-ray Physics, within the Göttingen Graduate School for Neurosciences, Biophysics, and Molecular Biosciences (GGNB) of the Georg-August University School of Science (GAUSS), Göttingen, Germany.

- 2013 - 2015 MSc in Physics, University of Milano-Bicocca, Milano (Italy)

- MSc thesis: "The role of mechanical forces in the robustness of C. elegans embryonic development", supervised by Prof. Dr. Giuseppe Chirico and Dr. Jeroen van Zon, at the AMOLF Institute, Amsterdam, The Netherlands.

- 2010 - 2013 BSc in Physics, University of Milano-Bicocca, Milano (Italy)

- BSc thesis: "Internalization study of metal nanoparticles in cell by optical microscopy in reflection with correlative and imaging methods", supervised by Prof. Dr. Maddalena Collini, at the Milano-Bicocca Biophysics and Biophotonics Group, Milano, Italy.

- 2005 - 2010 Liceo Scientifico Piero Bottoni, Milano (Italy) 